\title{
Carbonyl-Directed Aliphatic Fluorination: A Special Type of Hydrogen Atom Transfer Beats Out Norrish II
}

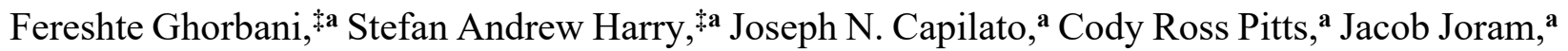
Garvin N. Peters, ${ }^{\mathbf{a}}$ John D. Tovar, ${ }^{\mathbf{a}}$ Ivor Smajlagic, ${ }^{\mathbf{b}}$ Maxime A. Siegler, ${ }^{\mathbf{a}}$ Travis Dudding, ${ }^{* \mathbf{b}}$ and Thomas Lectka*a

${ }^{a}$ Department of Chemistry, Johns Hopkins University, 3400 N. Charles St., Baltimore, MD, 21218, United States

${ }^{b}$ Department of Chemistry, Brock University, 1812 Sir Isaac Brock Way, St. Catharines, ON L2S 3A1, Canada

$\$$ F.G. and S.A.H. contributed equally to this work.

\section{Contents:}

General Information, S2-S3

General Fluorination Procedures, S3

${ }^{13} \mathrm{C} /{ }^{12} \mathrm{C}$ Experiments, S3-S5

Electrolysis Procedure, S5

CV Procedure, $S 5$

Polar Effect Experiment, S6

Borane Initiation Procedure, S6

Ferrioxalate Actinometry Experiment, S7

Selectfluor Derivative Competition, $\mathrm{S8}$

Intermolecular Competition H/D Experiments, S8

Rate Study 2-Ethyl-cyclohexan-1-one H/D Experiments, S9-S10

Synthetic Probe Experiment, S10-S11

Wurster's Blue Experiment, S11

Syntheses and Characterization Data, S11-S17

Spectral Data, S18-S33

Single Crystal X-ray Crystallography, S34-S35

Computational Data, S35-S169

UV-Vis Spectrum, S169

References, S170-S171 


\section{General Information}

Unless otherwise stated, all reactions were carried out under strictly anhydrous conditions and $\mathrm{N}_{2}$ atmosphere. All solvents were dried and distilled by standard methods. All ${ }^{1} \mathrm{H}$ NMR spectra were acquired on a $400 \mathrm{MHz} \mathrm{NMR}$ spectrometer in $\mathrm{CDCl}_{3},{ }^{19} \mathrm{~F}$ NMR spectra were acquired on a 300 $\mathrm{MHz}$ NMR spectrometer in $\mathrm{CD}_{3} \mathrm{CN}$ or $\mathrm{CDCl}_{3}$, and ${ }^{13} \mathrm{C}$ NMR spectra were acquired on a $400 \mathrm{MHz}$ NMR spectrometer in $\mathrm{CDCl}_{3}$. The ${ }^{1} \mathrm{H},{ }^{13} \mathrm{C}$, and ${ }^{19} \mathrm{~F}$ NMR chemical shifts are given in parts per million $(\delta)$ with respect to an internal tetramethylsilane (TMS, $\delta=0.00 \mathrm{ppm}$ ) standard and/or 3-chlorobenzotrifluoride $\left(\delta=-64.2 \mathrm{ppm}\right.$ relative to $\left.\mathrm{CFCl}_{3}\right) .{ }^{1} \mathrm{NMR}$ data are reported in the following format: chemical shift (integration, multiplicity $(\mathrm{s}=$ singlet, $\mathrm{d}=$ doublet, $\mathrm{t}=$ triplet, $\mathrm{q}=$ quartet, $\mathrm{m}=$ multiplet), coupling constants $(\mathrm{Hz})$ ). Spectral data were processed with Bruker software. Photochemical reactions were run in front of White LEDs $(400 \mathrm{~nm})$ or in a Rayonet reactor using $300 \mathrm{~nm}$ bulbs. X-ray crystallographic data: All reflection intensities were measured at $110(2) \mathrm{K}$ using a SuperNova diffractometer (equipped with Atlas detector) with Mo Ka radiation $(\lambda=0.71073 \AA$ ) under the program CrysAlisPro (Version CrysAlisPro 1.171.39.29c, Rigaku OD, 2017). The same program was used to refine the cell dimensions and for data reduction. The structure was solved with the program SHELXS-2018/3 (Sheldrick, 2018) and was refined on $F^{2}$ with SHELXL-2018/3 (Sheldrick, 2018). Numerical absorption correction based on gaussian integration over a multifaceted crystal model was applied using CrysAlisPro. The temperature of the data collection was controlled using the system Cryojet (manufactured by Oxford Instruments). The $\mathrm{H}$ atoms were placed at calculated positions using the instructions AFIX 43 with isotropic displacement parameters having values $1.2 U_{\text {eq }}$ of the attached $\mathrm{C}$ atoms. Density functional theory calculations were performed using the software package Gaussian 09, Revision E. 01. ${ }^{2}$ All optimizations were performed applying the B3LYP functional (unrestricted) that includes Becke's three-parameter gradient-corrected exchange functional ${ }^{3}$ and the LYP correlation functional ${ }^{4}$ of Lee, Yang, and Parr along with the 6-31G(d) basis set. The optimized geometries were verified as transition state structures (one imaginary frequency) by frequency calculations. Intrinsic reaction coordinate (IRC) calculations ${ }^{5,6}$ were performed to confirm that all transition state structures were linked to relevant minima. The energies of the UB3LYP/6-31G(d) optimized structures were

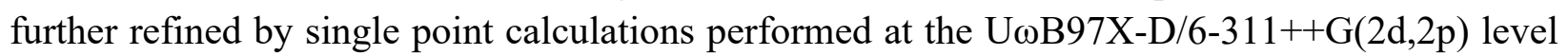
of theory using the integral equation formalism polarizable continuum model (IEFPCM) with the default parameters of acetonitrile $(\varepsilon=37.5)$ to account for solvent. ${ }^{7}$ The final reported Gibbs free energies are the summed thermal corrections to the Gibbs free energies (temperature $=298.15 \mathrm{~K}$ ) computed at the lower (UB3LYP/6-31G(d)) level of theory and electronic energies from single

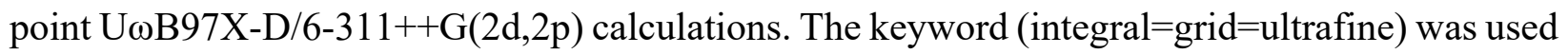
for all calculations. The respective natural bond orbital (NBO) charges and second-order perturbation theory donor-acceptor interactions were obtained from the optimized transition state involving carbon C46 (bottom hydrogen atom) computed at the UB3LYP/6-31+G(d,p) level of theory. The 3D images of all optimized geometries were generated with CYLview, ${ }^{8}$ and GaussView ${ }^{9}$ was used to construct all structures prior to optimization and to visualize the output from the Gaussian 09 calculations. The reported spin orbital densities (isovalue $=-0.0045$ ) and 
molecular electrostatic potential (MEP) surfaces were calculated using the UB3LYP-D3 functional with a 6-311++G(2d,2p) basis set using the program Jaguar of the Schrödinger software package. ${ }^{10}$ Characterization of 2 -ethyl cyclohexan-1-one, ${ }^{11} \quad 3 \beta, 20 \beta$-diacetoxy-5 $\alpha$-pregnen-7-one, ${ }^{12} \quad 1$ chloromethyl-4-aza-1-azoniabicyclo[2.2.2] octane tetrafluoroborate, ${ }^{13}$ 1-methyl-4-aza-1azoniabicyclo[2.2.2] octane trifluoromethanesulfonate, ${ }^{14}$ 1-fluoro-4-methyl-1,4diazoniabicyclo[2.2.2] octane ditetrafluoroborate, ${ }^{15}$ and 1-fluoro-4-methyl-1,4diazoniabicyclo[2.2.2] octane ditrifluoromethanesulfonate ${ }^{15}$ were consistent with literature precedent.

\section{General 400 nm Fluorination Procedure:}

Selectfluor (177 mg, $0.50 \mathrm{mmol})$, benzil $(5.0 \mathrm{mg}, 0.025 \mathrm{mmol})$, and the substrate $(0.25 \mathrm{mmol})$ were added to an oven-dried $\mu \omega$ vial equipped with a stir bar; the vial was then sealed with a cap with a septum using a crimper and evacuated/refilled with $\mathrm{N}_{2}$ multiple times. Anhydrous $\mathrm{MeCN}$ $(4 \mathrm{~mL})$ was added, and the reaction mixture was irradiated with a cool white LED work light while stirring. After $14 \mathrm{~h}$, a $0.3 \mathrm{~mL}$ aliquot was taken for ${ }^{19} \mathrm{~F}$ NMR yield determination. Then the reaction mixture was diluted with EtOAc, filtered through Celite, and concentrated. The crude reaction mixture was purified via gradient column chromatography on silica gel eluting with EtOAc/hexanes.

\section{General 300 nm Fluorination Procedure:}

Selectfluor $(97 \mathrm{mg}, 0.28 \mathrm{mmol})$ and the substrate $(0.13 \mathrm{mmol})$ were added to an oven-dried $\mu \omega$ vial equipped with a stir bar; the vial was then sealed with a cap with a septum using a crimper and evacuated/refilled with $\mathrm{N}_{2}$ multiple times. Anhydrous $\mathrm{CH}_{3} \mathrm{CN}(6 \mathrm{~mL})$ was added, and the reaction mixture was irradiated at $300 \mathrm{~nm}$ in a Rayonet reactor while stirring. After $4 \mathrm{~h}$, a $0.3 \mathrm{~mL}$ aliquot was taken for ${ }^{19} \mathrm{~F}$ NMR yield determination, and the rest of the reaction mixture was poured over $\mathrm{Et}_{2} \mathrm{O}$, filtered through Celite, and concentrated. The crude reaction mixture was purified initially via gradient column chromatography on silica gel eluting with EtOAc/hexanes. Analytical purity was obtained via subsequent HPLC purification.

\section{Intermolecular Competition Procedure for ${ }^{13} \mathrm{C} /{ }^{12} \mathrm{C}$ :}

Selectfluor (153 mg, $0.43 \mathrm{mmol})$, diphenylethanedione $(7.5 \mathrm{mg}, 0.036 \mathrm{mmol}$ ), and diphenylethanedione- ${ }^{13} \mathrm{C}_{2}(7.6 \mathrm{mg}, 0.036 \mathrm{mmol})$ were added to an oven-dried microwave vial equipped with a stir bar. Then, the vial was sealed via crimper with a cap with septum; it was evacuated and refilled with $\mathrm{N}_{2}$ multiple times. Anhydrous $\mathrm{CH}_{3} \mathrm{CN}(3 \mathrm{~mL})$ was added via syringe under $\mathrm{N}_{2}$. The reaction mixture was stirred in front of LEDs overnight. Remaining starting material 
ratios were determined by mass spectrometry analysis; NMR ratios/yields were carefully determined to be in agreement with mass spectrometry analysis. The reaction was run in triplicate (1.05, 1.06, and 1.06) and averaged 1.06 (see Table S1; calculations for a single experiment).

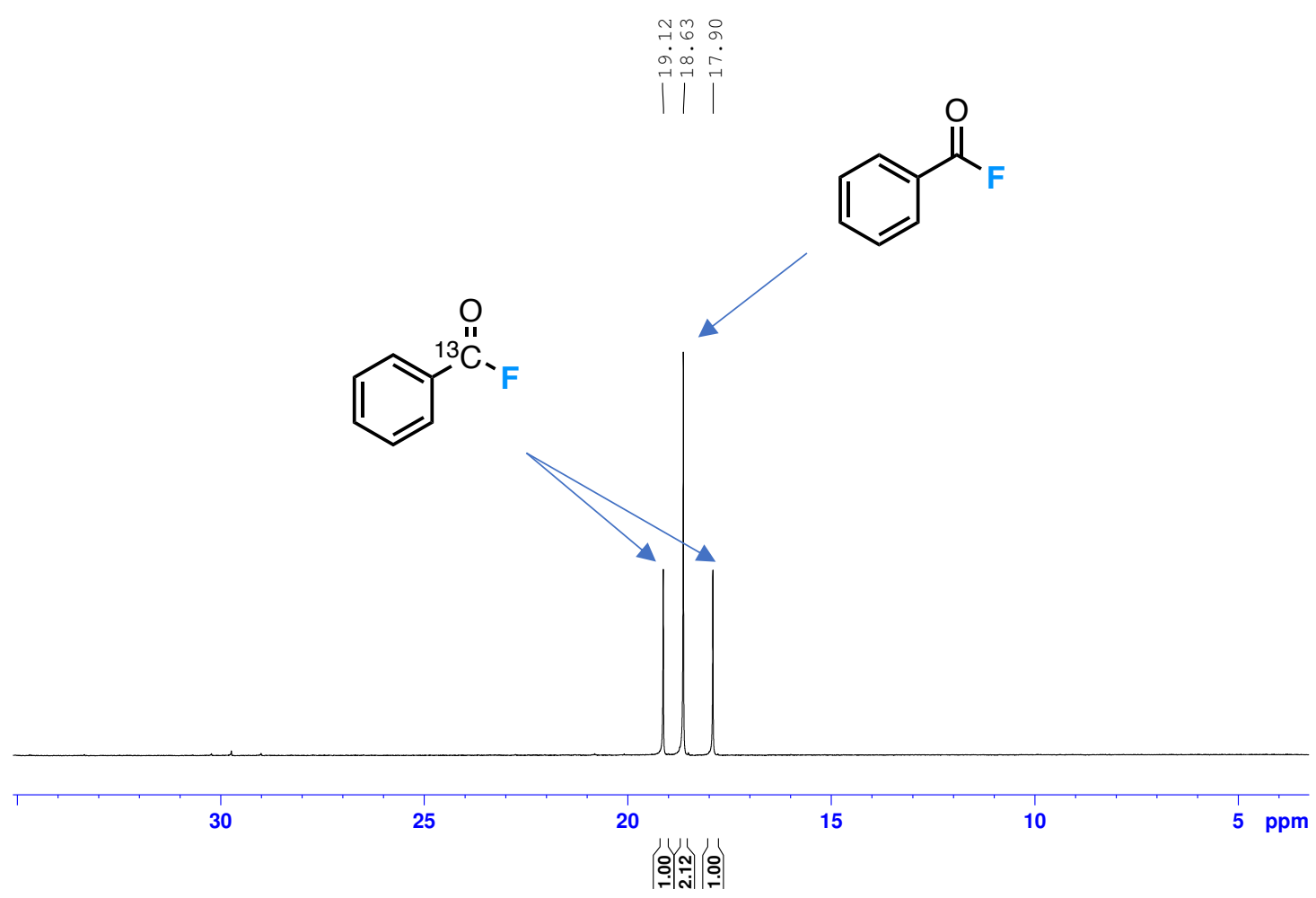

Figure S1. ${ }^{19} \mathrm{~F}\left\{{ }^{1} \mathrm{H}\right\}$ NMR spectrum of intermolecular ${ }^{13} \mathrm{C} /{ }^{12} \mathrm{C}$ competition.

\begin{tabular}{|c|c|c|c|c|c|c|}
\hline Replicate \# & Intensity $\mathrm{m} / \mathrm{z}=\mathbf{2 1 0}$ & Intensity $\mathrm{m} / \mathrm{z}=212$ & $\begin{array}{l}\text { Total Intensity } \\
210+212\end{array}$ & $\% 210$ in Total & \%212 in Total & $\begin{array}{c}\text { Ratio Intensity } \\
210 / 212\end{array}$ \\
\hline 1 & 48.07 & 50.30 & 98.37 & 48.87 & 51.13 & 0.9557 \\
\hline 2 & 48.62 & 50.16 & 98.78 & 49.22 & 50.78 & 0.9693 \\
\hline 3 & 41.55 & 44.56 & 86.11 & 48.25 & 51.75 & 0.9325 \\
\hline 4 & 41.25 & 42.76 & 84.01 & 49.10 & 50.90 & 0.9647 \\
\hline \multirow[t]{4}{*}{5} & 49.57 & 50.42 & 99.99 & 49.57 & 50.43 & 0.9831 \\
\hline & & & Mean & 49.00 & 51.00 & 0.9610 \\
\hline & & & StDev & 0.492 & 0.492 & 0.0188 \\
\hline & & & \%sStdDev & 1.003 & 0.964 & 1.958 \\
\hline
\end{tabular}

Table S1. Calculations to determine ratio of ${ }^{13} \mathrm{C} /{ }^{12} \mathrm{C}$ species via mass spectroscopy.

Procedure for MS analysis: 5 replicate measurements were taken, with each measurement being an average of the first 100 scans. The relative intensities of the mass peaks at 210.2 and 212.2 were determined, as compared to base peak in the mass spectrum. For each measurement, the relative intensities of the 210.2 and 212.2 peaks were added to get the total intensity of both peaks. 
The relative percentage for each peak was determined, along with the ratio of [210.2]/[212.2]. Mean, standard deviation and relative standard deviation were determined from 5 replicate runs.

\section{Electrolysis Procedure:}

A mixture of Selectfluor (106 $\mathrm{mg}, \quad 0.30 \quad \mathrm{mmol})$, 1-chloromethyl-4-aza-1azoniabicyclo[2.2.2] octane tetrafluoroborate $(168 \mathrm{mg}, 0.675 \mathrm{mmol})$, steroid substrate $4(104 \mathrm{mg}$, $0.25 \mathrm{mmol})$, and tetrabutylammonium hexafluorophosphate $(3.14 \mathrm{~g}, 8.10 \mathrm{mmol})$ were added to a flame-dried round-bottom flask equipped with a stir bar; the flask was then evacuated/refilled with $\mathrm{N}_{2}$ multiple times. Anhydrous $\mathrm{CH}_{3} \mathrm{CN}(81 \mathrm{~mL})$ was added, and the reaction mixture was stirred vigorously before being transferred to the bulk electrolysis glass cell vial. Working electrode (reticulated vitreous carbon - anode), the vial cap equipped with reference electrode (silver wire submersed in $0.01 \mathrm{M} \mathrm{AgNO}_{3} / 0.1 \mathrm{M}$ n- $\mathrm{Bu}_{4} \mathrm{NPF}_{6}$ in anhydrous $\mathrm{MeCN}$ ) and auxiliary electrode (coiled platinum - cathode) were all inserted into the mixture. The reaction mixture was electrolyzed at a constant potential of $1.8 \mathrm{~V}$ for 6 hours. After the reaction, a $2.0 \mathrm{~mL}$ aliquot was taken for ${ }^{1} \mathrm{H}$ NMR yield determination. The rest of the reaction mixture was concentrated in vacuo, diluted with $\mathrm{Et}_{2} \mathrm{O}$, and filtered through Celite. The crude reaction mixture was purified via gradient column chromatography on silica gel eluting with EtOAc/hexanes. A BASi ${ }^{\circledR}$ Bulk Electrolysis Cell Kit was used.

\section{Cyclic Voltammetry General Procedure:}

The oxidation potential was measured for a $2.5 \mathrm{mM}$ solution of the substrate in $0.1 \mathrm{M}$ electrolyte $\mathrm{TBABF}_{4}$ in $\mathrm{MeCN}$. A conventional three-electrode configuration was used, platinum disk (working electrode), platinum wire (counter electrode), and $\mathrm{Ag} / \mathrm{AgNO}_{3}$ reference electrode. The cycling was performed by applying a linear potential scan at a sweep rate of $10 \mathrm{mV} \mathrm{s}^{-1}$, and it was repeated until a reproducible cyclic voltammetry curve was obtained before the measurement curves were recorded. Nitrogen was purged through the solution for several minutes before the tests were performed. Potentials are reported relative to the $\mathrm{Ag} / \mathrm{Ag}+$ redox couple $(0.00 \mathrm{~V})$ against which the $\mathrm{Fc} / \mathrm{Fc}+$ couple was measured to be $0.221 \mathrm{~V}$. 


\section{Polar Effect Experiment:}

As per the general $400 \mathrm{~nm}$ fluorination protocol with substrate 2-heptanone.

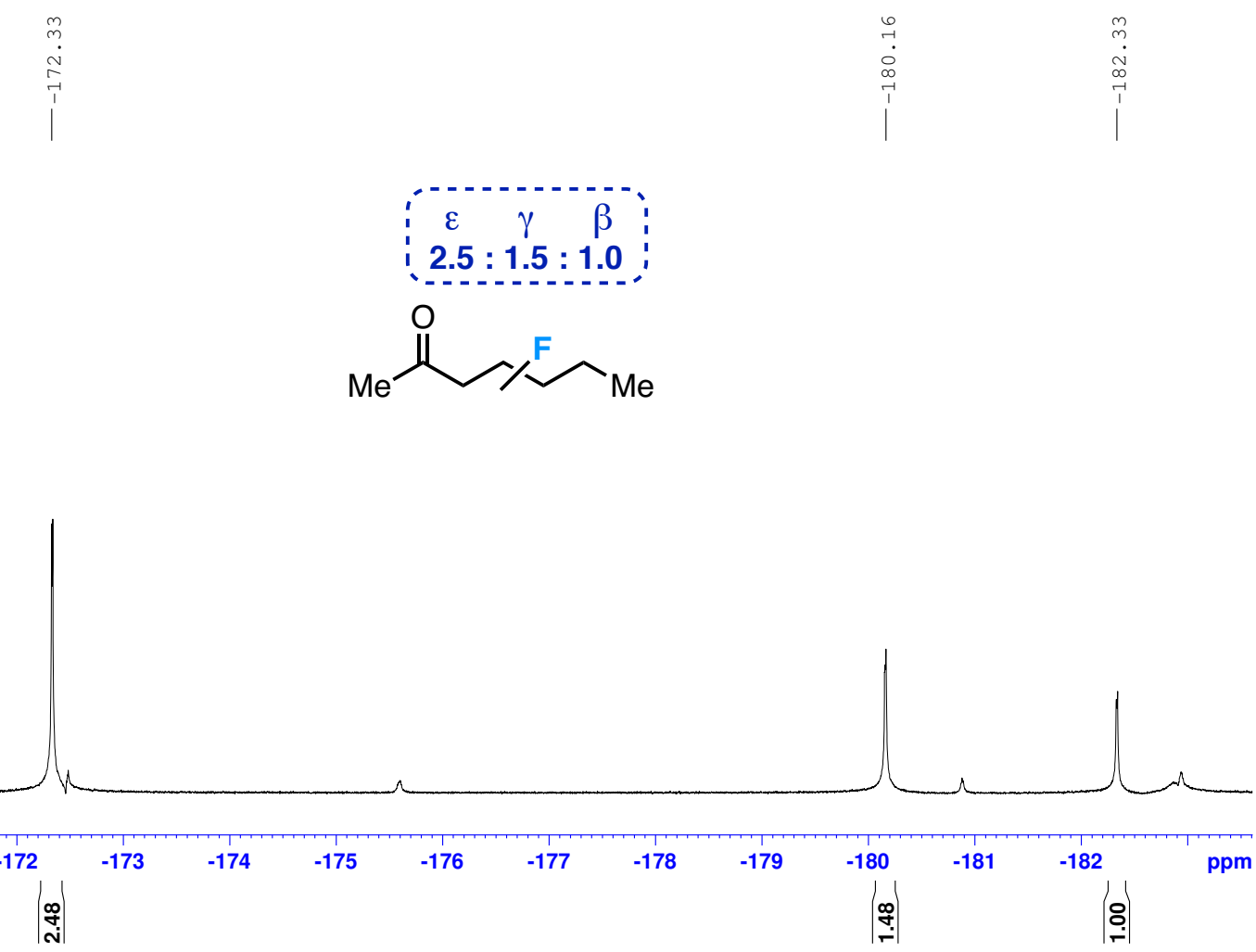

Figure S2. ${ }^{19} \mathrm{~F}\left\{{ }^{1} \mathrm{H}\right\}$ NMR spectrum of product ratios for the polar effect experiment.

\section{Borane Initiation Procedure:}

Selectfluor (133 mg, $0.375 \mathrm{mmol})$ was added to an oven-dried microwave vial equipped with a stir bar under $\mathrm{N}_{2}$. Then, anhydrous $\mathrm{CH}_{3} \mathrm{CN}(3.0 \mathrm{~mL})$ was added to the reaction flask, and the solution was stirred vigorously at room temperature. Steroid substrate $1(78.0 \mathrm{mg}, 0.187 \mathrm{mmol})$ was added followed by a $1.0 \mathrm{M}$ triethylborane solution in hexanes $(0.05 \mathrm{~mL}, 0.034 \mathrm{mmol})$. The reaction mixture was stirred overnight. After the reaction, an aliquot was taken for ${ }^{19} \mathrm{~F}$ NMR yield determination. 


\section{Ferrioxalate Actinometry: ${ }^{16}$}

A $0.15 \mathrm{M}$ solution of ferrioxalate was prepared by dissolving $2.20 \mathrm{~g}$ of potassium ferrioxalate hydrate in $30 \mathrm{~mL}$ of $0.05 \mathrm{M} \mathrm{H}_{2} \mathrm{SO}_{4}$. A buffered solution of phenanthroline was prepared by dissolving $50 \mathrm{mg}$ of phenanthroline and $11.2 \mathrm{~g}$ of sodium acetate in $50 \mathrm{~mL}$ of $0.5 \mathrm{M} \mathrm{H}_{2} \mathrm{SO}_{4}$. The two solutions were wrapped in foil and stored until further use. To determine the photon flux of the spectrophotometer, $3.5 \mathrm{~mL}$ of the ferrioxalate solution was placed in a cuvette and irradiated for 90.0 seconds at $\lambda=436 \mathrm{~nm}$ with an emission slit width at $10.0 \mathrm{~nm}$. Thereafter, $0.61 \mathrm{~mL}$ of the phenanthroline solution was added. The solution was then wrapped in foil and sat for $1 \mathrm{~h}$ to allow the ferrous ions to completely coordinate to the phenanthroline. The absorbance of the solution was measured at $510 \mathrm{~nm}$. Also, a non-irradiated sample was prepared and the absorbance at 510 $\mathrm{nm}$ was measured. Moles of $\mathrm{Fe}^{2+}$ were calculated using Equation 1.

Equation 1: $\mathrm{mol} \mathrm{Fe} \mathrm{e}^{2+}=\frac{\mathrm{V} \cdot \Delta \mathrm{A}}{\mathrm{l} \cdot \varepsilon}$

Note: $\mathrm{V}$ is the total volume $(0.00411 \mathrm{~L})$ of the solution after addition of phenanthroline, $\Delta \mathrm{A}$ is the difference in absorbance at $510 \mathrm{~nm}$ between the irradiated and non-irradiated solutions, 1 is the path length $(1.000 \mathrm{~cm})$, and $\varepsilon$ is the molar absorptivity at $510 \mathrm{~nm}\left(11,100 \mathrm{~L} \mathrm{~mol}^{-1} \mathrm{~cm}^{-1}\right)$. The photon flux can be calculated using Equation 2.

Equation 2: photon flux $=\frac{\mathrm{mol} \mathrm{Fe}^{2+}}{\Phi \cdot \mathrm{t} \cdot \mathrm{f}}$

Where $\Phi$ is the quantum yield for the ferrioxalate actinometer $(1.01$ for a $0.15 \mathrm{M}$ solution at $\lambda=$ $436 \mathrm{~nm}), \mathrm{t}$ is the time $(90.0 \mathrm{~s})$, and $\mathrm{f}$ is the fraction of light absorbed at $\lambda=436 \mathrm{~nm}(0.998$, vide infra). The photon flux was calculated to be $9.61 \times 10^{-9}$ einstein $\mathrm{s}^{-1}$.

The absorbance of the above ferrioxalate solution at $436 \mathrm{~nm}$ was measured to be 2.72 . The fraction of light absorbed (f) by this solution was calculated using Equation 3, where A is the measured absorbance at $436 \mathrm{~nm}$.

Equation 3: $\mathrm{f}=1-10^{-\mathrm{A}}$

Substrate 6 ( $0.25 \mathrm{mmol}, 1$ equiv), Selectfluor ( $0.50 \mathrm{mmol}, 2$ equiv), and benzil (0.025 mmol, 10 mol\%) were added to a dry cuvette. The cuvette was then capped with a PTFE stopper and flushed with nitrogen gas. Then, $4.0 \mathrm{~mL}$ of degassed $\mathrm{MeCN}$ was added and the sample was stirred and irradiated $(\lambda=436 \mathrm{~nm}$, slit width $=10.0 \mathrm{~nm})$ for $2400 \mathrm{~s}$. The yield of product formed was determined by ${ }^{19} \mathrm{~F}$ NMR based on a 4-bromobenzotrifluoride standard. The quantum yield was determined using Equation 4. The fraction of light absorbed (f) was found to be 0.08 at the aforementioned reaction conditions.

Equation 4: $\Phi=\frac{\text { mol product }}{\text { flux } \cdot \mathrm{t} \cdot \mathrm{f}}$

Experiment example: $104 \mathrm{mg}(0.25 \mathrm{mmol})$ substrate 6, $177 \mathrm{mg}(0.50 \mathrm{mmol})$ Selectfluor, $5 \mathrm{mg}$ $(0.025 \mathrm{mmol})$ benzil, $4.0 \mathrm{~mL} \mathrm{MeCN}$ after $2400 \mathrm{~s}$ yielded $13 \%$ of the desired fluorinated product and $\Phi(13 \%)=18$. 


\section{Competition Experiment: Selectfluor vs. Compound 13-b}

In separate oven-dried $\mu \omega$ vials equipped with stirs bars, the fluorinating reagent $(0.172 \mathrm{mmol})$, benzil $(0.0006 \mathrm{mmol})$, and substrate $4(0.030 \mathrm{mmol})$ were added. The vial was then sealed with a cap with septum using a crimper and evacuated/refilled with $\mathrm{N}_{2}$ multiple times. Anhydrous $\mathrm{MeCN}$ $(3 \mathrm{~mL})$ was added, and the reaction mixture was irradiated with a cool white LED work light while stirring. After an allotted amount of time, a $0.3 \mathrm{~mL}$ aliquot was taken for ${ }^{19} \mathrm{~F}$ NMR yield determination.

\section{Intermolecular Competition Procedure for 2-Ethyl-cyclohexan-1-one H/D:}

Selectfluor (133 mg, $0.375 \mathrm{mmol}), 2$-ethyl-cyclohexan-1-one (164.5 mg, $1.25 \mathrm{mmol})$, 2-ethyl-D cyclohexan-1-one $(158.1 \mathrm{mg}, 1.25 \mathrm{mmol})$, and benzil $(5.0 \mathrm{mg}, 0.025 \mathrm{mmol})$ were added to an oven-dried microwave vial equipped with a stir bar. Then, the vial was sealed via crimper with a cap with septum; it was evacuated and refilled with $\mathrm{N}_{2}$ multiple times. Anhydrous $\mathrm{CH}_{3} \mathrm{CN}(5 \mathrm{~mL})$ was then added and the reaction mixture was stirred in front of LEDs for $48 \mathrm{~h}$. Product ratios $\left(\left[\mathrm{P}_{\mathrm{H}}\right] /\left[\mathrm{P}_{\mathrm{D}}\right]\right)$ were determined by ${ }^{19} \mathrm{~F}$ NMR analysis of a sample composed of a $0.3 \mathrm{~mL}$ aliquot from the reaction flask and $0.2 \mathrm{~mL}$ of a dilute solution of 3-chlorobenzotrifluoride (internal standard) dissolved in $\mathrm{CD}_{3} \mathrm{CN}$. The reaction was run in duplicate (3.3 and 3.5); KIE average: 3.4.

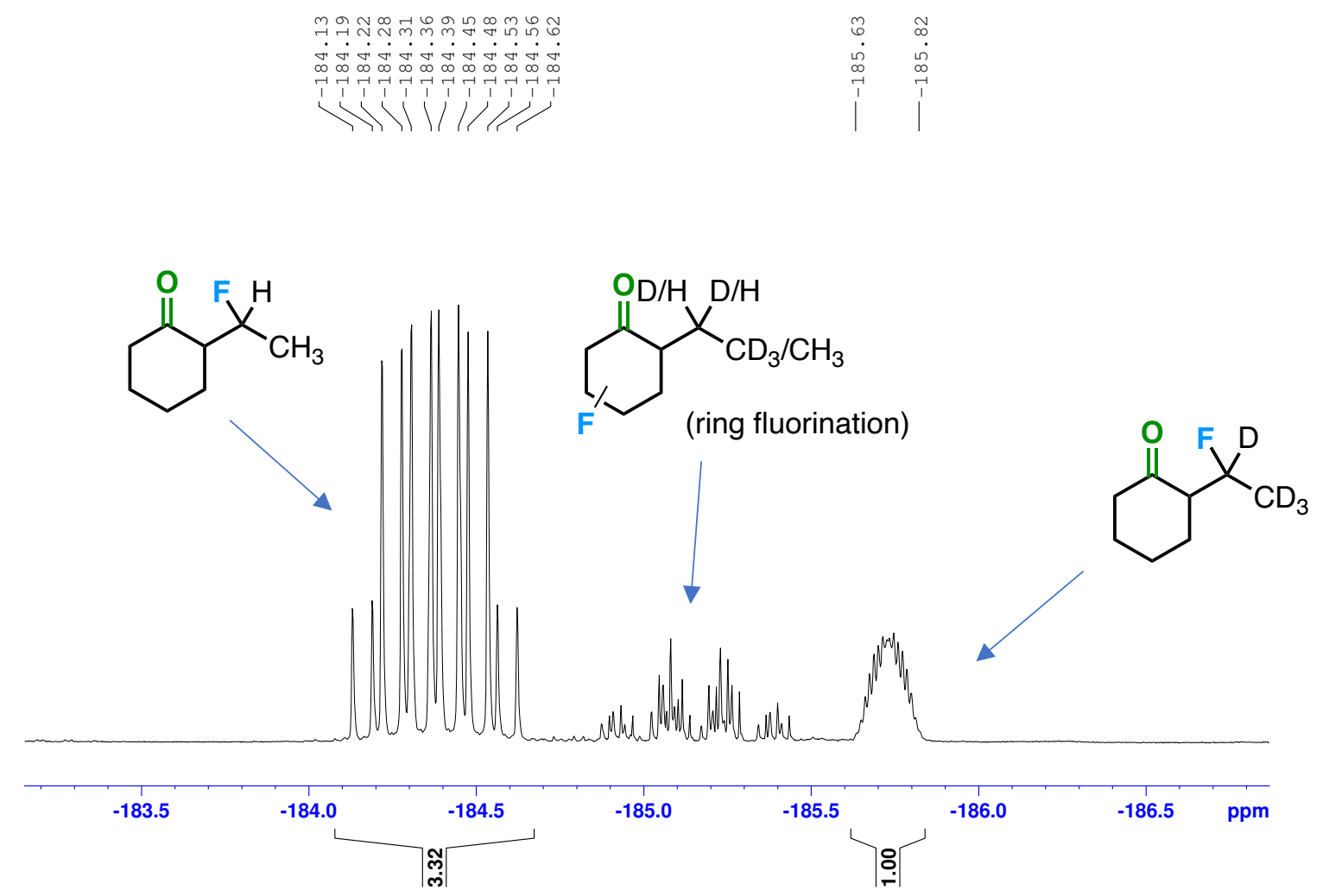

Figure S3. ${ }^{19} \mathrm{~F}$ NMR spectrum of intermolecular H/D competition experiment. 


\section{Rate Study of 2-Ethyl-cyclohexan-1-one H/D}
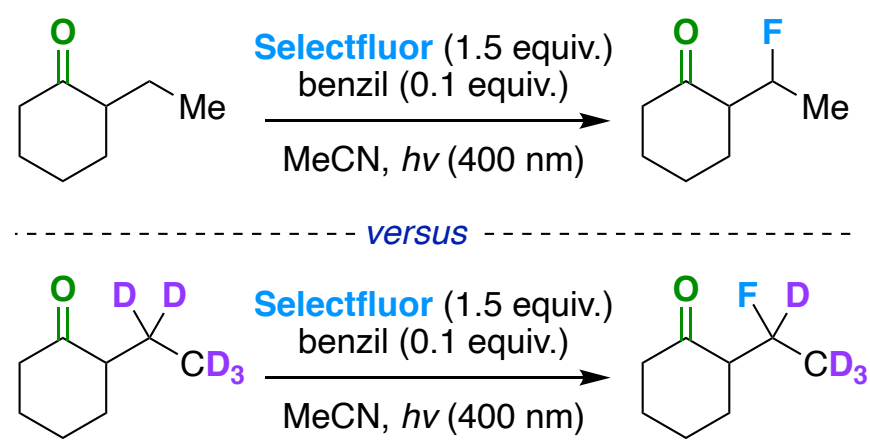

Selectfluor (232 mg, $0.654 \mathrm{mmol})$, benzil (10.0 mg, $0.050 \mathrm{mmol})$, and 2-ethyl-cyclohexan-1-one $(55.0 \mathrm{mg}, 0.435 \mathrm{mmol})$ or 2-ethyl-D 5 cyclohexan-1-one $(57.0 \mathrm{mg}, 0.435 \mathrm{mmol})$ were added to an oven-dried microwave vial equipped with a stir bar. Then, the vial was sealed via crimper with a cap with septum; it was evacuated and refilled with $\mathrm{N}_{2}$ multiple times. Anhydrous $\mathrm{CH}_{3} \mathrm{CN}(6 \mathrm{~mL})$ was then added and the reaction mixture was stirred in front of LEDs. Aliquots were taken at 5 or 15-minute (depending on the isotopomer) intervals for analysis. Product concentrations were determined by ${ }^{19} \mathrm{~F}\left\{{ }^{1} \mathrm{H}\right\}$ NMR analysis of a sample composed of a $0.3 \mathrm{~mL}$ aliquot from the reaction flask and $0.1 \mathrm{~mL}$ of a dilute solution of 4-bromobenzotrifluoride (internal standard) dissolved in $\mathrm{CD}_{3} \mathrm{CN}$. Initial rates were determined and a KIE of 3.7 was observed.

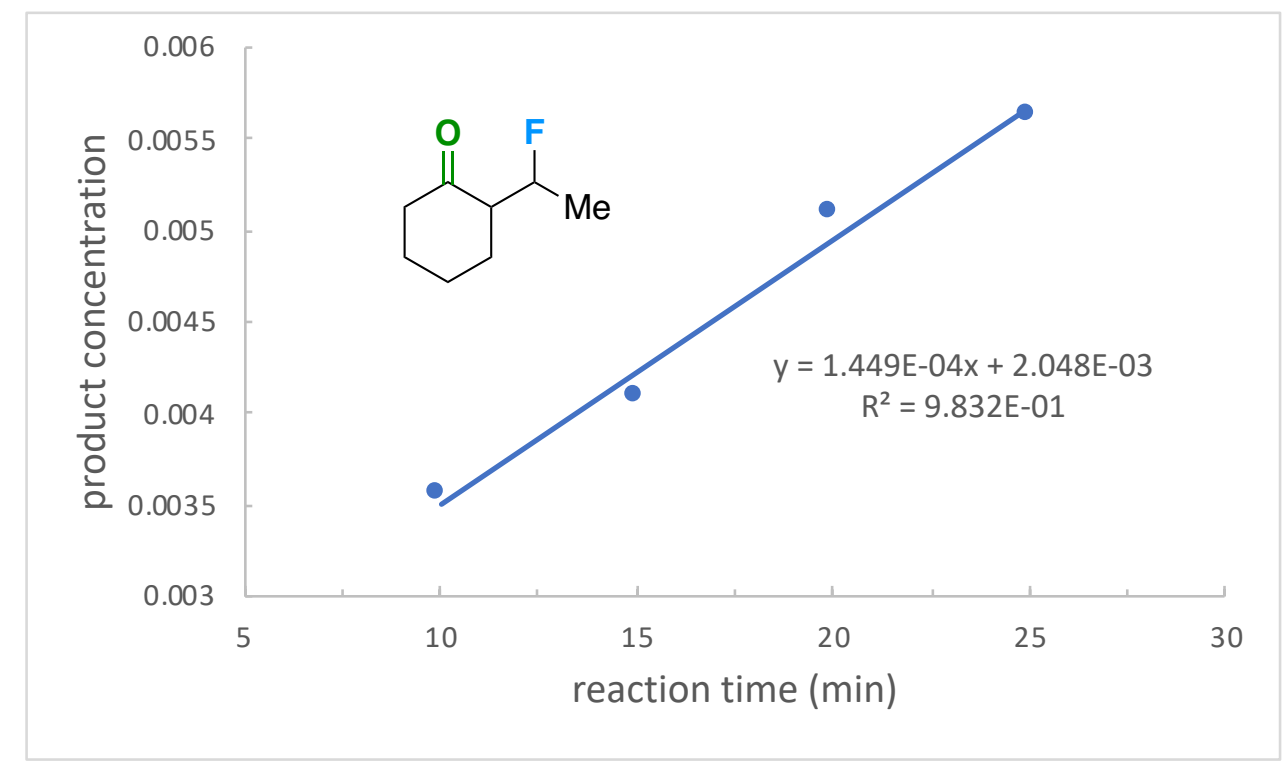

Figure S4. Plot for the initial rate of formation of 2-(1-fluoroethyl)cyclohexan-1-one. 


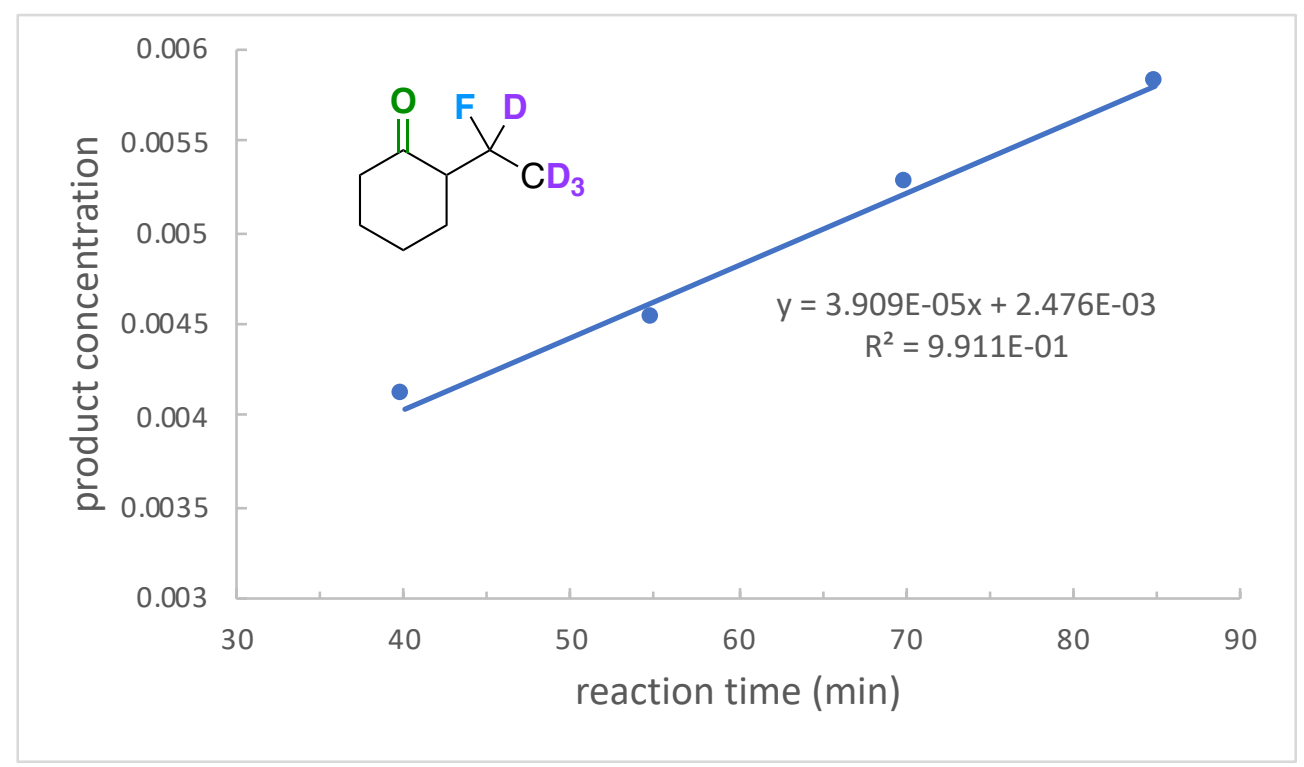

Figure S5. Plot for the initial rate of formation of 2-(1-fluoroethyl-1,2,2,2- $\left.d_{4}\right)$ cyclohexan-1-one.

\section{Synthetic Probe Experiment: Compound 17}

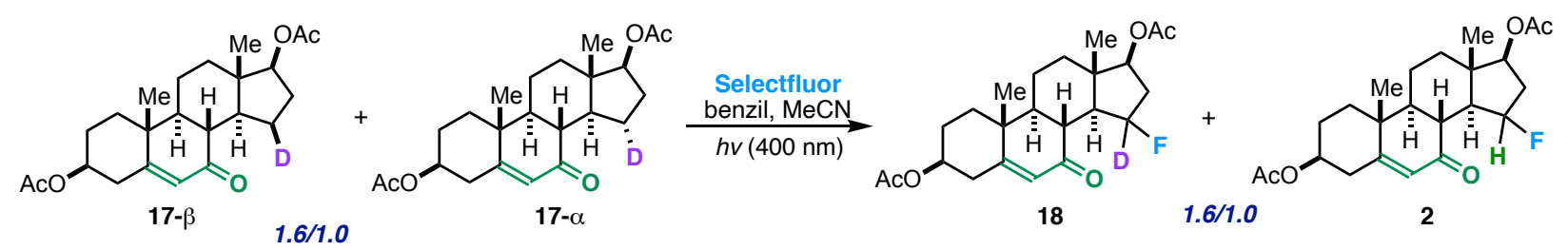

Compound 17 was subjected to the general $400 \mathrm{~nm}$ fluorination conditions. The resultant crude mixture was analyzed via ${ }^{19} \mathrm{~F}$ NMR to determine product ratios. 


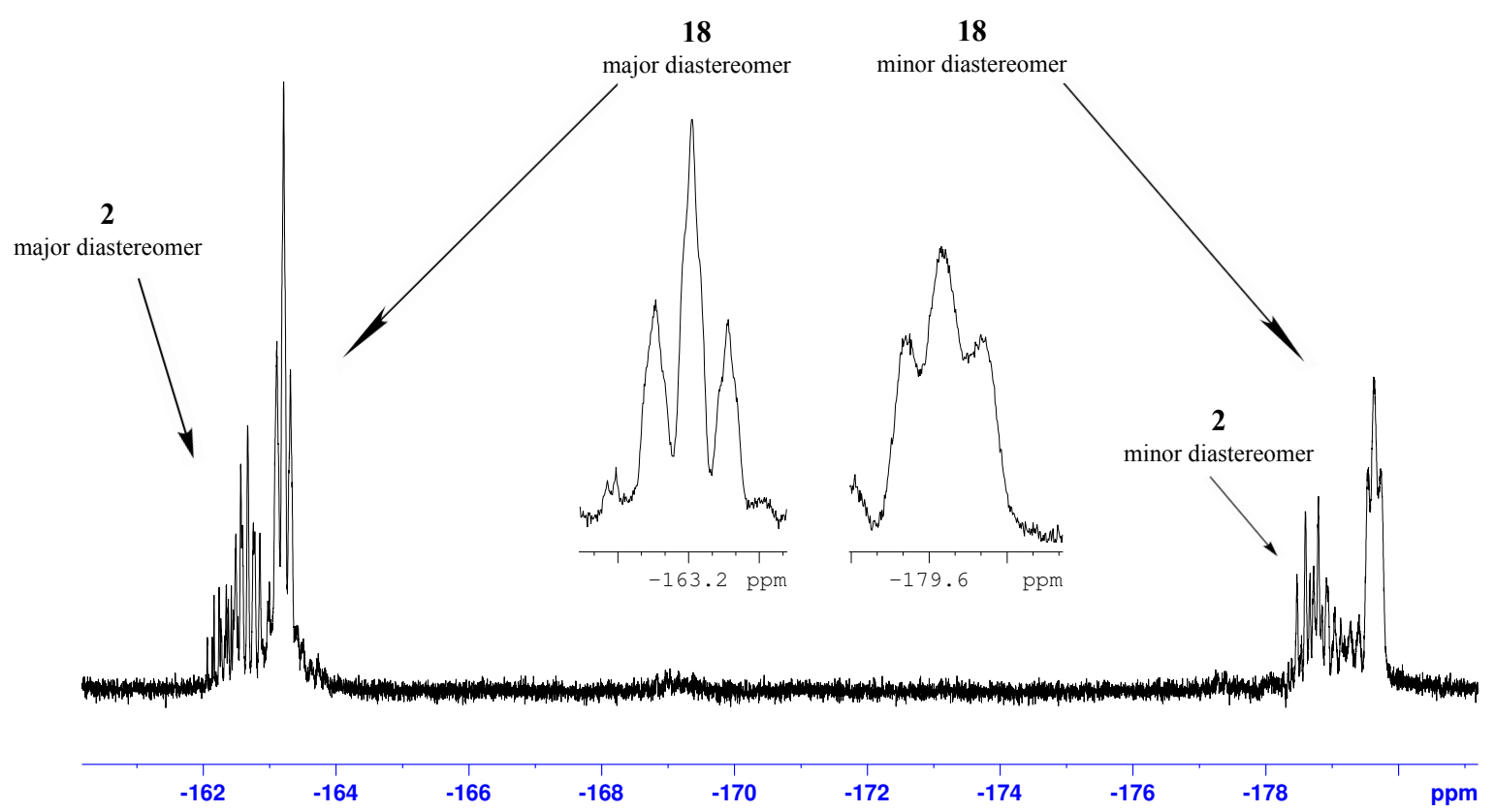

Figure S6. ${ }^{19} \mathrm{~F}$ NMR spectrum of compound 2 and 18.

\section{Wurster's Blue Experiment}

$N, N, N^{\prime}, N^{\prime}$-Tetramethyl-p-phenylenediamine (50 mg, $0.304 \mathrm{mmol}$ ) was added to an oven-dried microwave vial equipped with a stir bar under $\mathrm{N}_{2}$. Then, anhydrous $\mathrm{CH}_{3} \mathrm{CN}(2.0 \mathrm{~mL})$ was added to the reaction flask, and the solution was stirred at room temperature. Selectfluor (119 mg, 0.335 mmol) in $\mathrm{CH}_{3} \mathrm{CN}(2.0 \mathrm{~mL})$ was added dropwise and the reaction mixture immediately turned deep blue. After stirring for 30 minutes an aliquot was taken and analyzed via ${ }^{1} \mathrm{H}$ NMR.

\section{Syntheses}

\section{$\underline{\text { 15S-fluoro-7-keto-cholesteryl acetate }}$}

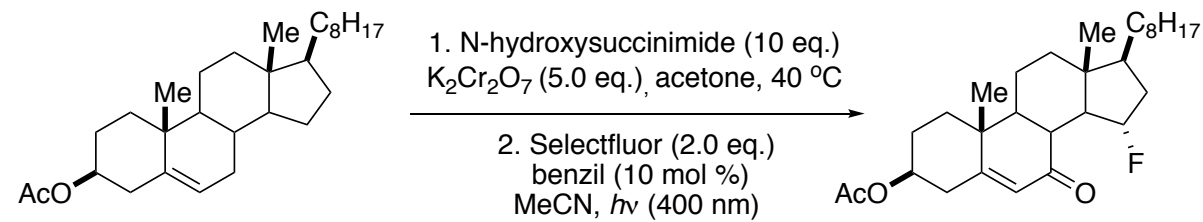


Cholesteryl acetate $(4.0 \mathrm{~g}, 9.3 \mathrm{mmol})$ was dissolved in a mixture of acetone $(475 \mathrm{~mL})$ and acetic acid $(50 \mathrm{~mL})$ in a round-bottom flask equipped with a stir bar and reflux condenser under $\mathrm{N}_{2}$. The reaction mixture was treated with $N$-hydroxysuccinimide ( $10.7 \mathrm{~g}, 93.0 \mathrm{mmol})$ and $\mathrm{K}_{2} \mathrm{Cr}_{2} \mathrm{O}_{7}(11.0$ g, $37.2 \mathrm{mmol}$ ), and then the reaction mixture was stirred at $40^{\circ} \mathrm{C}$ for $48 \mathrm{~h}$. The reaction mixture was cooled to rt, quenched with $10 \%$ aqueous sodium metabisulfite solution, filtered through Celite, and extracted into $\mathrm{Et}_{2} \mathrm{O}$. The combined organic layers were washed with saturated $\mathrm{NaHCO}_{3}$ and brine, and then dried with $\mathrm{MgSO}_{4}$ and concentrated. The crude residue was recrystallized in $\mathrm{MeOH}$ to provide 3 $\beta$-acetoxy-cholest-5-en-7-one (compound 6) $(3.28 \mathrm{~g}, 80 \%$ ). The aforementioned product was subjected to general $400 \mathrm{~nm}$ fluorination conditions.

White solid. ${ }^{1} \mathrm{H}$ NMR $\left(400 \mathrm{MHz}, \mathrm{CDCl}_{3}\right): \delta 5.85(\mathrm{~d}, J=2.0,1 \mathrm{H}), 5.30(\mathrm{dm}, J=53.4 \mathrm{~Hz}, 1 \mathrm{H})$, 4.80-4.72 (m, 1H), 2.66-2.60 (m, 1H), 2.53-2.46 (m, 1H), $2.33(\mathrm{t}, J=11.0,1 \mathrm{H}), 2.22-2.10(\mathrm{~m}$, $1 \mathrm{H}), 2.8(\mathrm{~s}, 3 \mathrm{H}), 2.05-1.86(\mathrm{~m}, 4 \mathrm{H}), 1.77-1.60(\mathrm{~m}, 4 \mathrm{H}), 1.56-1.45(\mathrm{~m}, 3 \mathrm{H}), 1.43-1.28(\mathrm{~m}, 5 \mathrm{H})$, $1.20-1.05(\mathrm{~m}, 7 \mathrm{H}), 0.95(\mathrm{~d}, J=6.5 \mathrm{~Hz}, 3 \mathrm{H}), 0.89(\mathrm{~d}, J=6.5 \mathrm{~Hz}, 3 \mathrm{H}), 0.88(\mathrm{~d}, J=6.7 \mathrm{~Hz}, 3 \mathrm{H})$, $0.74(\mathrm{~s}, 3 \mathrm{H}) .{ }^{13} \mathrm{C}\left\{{ }^{1} \mathrm{H}\right\}$ NMR $\left(100 \mathrm{MHz}, \mathrm{CDCl}_{3}\right): \delta 198.5,170.2,164.6,127.4,94.8,93.1,71.8$, $58.4,58.2,52.6,50.5,45.2,45.1,43.1,39.3,39.1,38.0,37.7,37.7,37.6,36.0,35.6,34.8,29.6$, $27.9,27.3,23.7,22.7,22.5,21.2,21.2,18.5,17.8,13.0 .{ }^{19} \mathrm{~F}$ NMR $\left(282 \mathrm{MHz}, \mathrm{CDCl}_{3}\right): \delta-160.5-$ (-161.0) (m). HRMS (ESI-Ion Trap) m/z: [M + H]+ calcd for 461.3431, found 461.3424. 


\section{2-Ethyl-D 5 -cyclohexan-1-one}

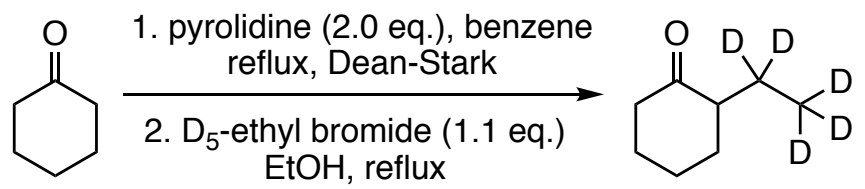

A solution of cyclohexanone $(7.0 \mathrm{~mL}, 72 \mathrm{mmol})$ and pyrrolidine $(12 \mathrm{~mL}, 143 \mathrm{mmol})$ in benzene $(33 \mathrm{~mL})$ was refluxed overnight with azeotropic removal of water. Benzene and excess pyrrolidine were removed under reduced pressure. To the resultant residual yellow oil were added ethyl bromide $(5.9 \mathrm{~mL}, 79 \mathrm{mmol})$ and ethanol $(9 \mathrm{~mL})$ at room temperature. After heating under reflux overnight, ethanol was removed under reduced pressure, and the resultant was heated under reflux with water $(27 \mathrm{~mL})$ for $3 \mathrm{~h}$. After cooling, the mixture was extracted into $\mathrm{Et}_{2} \mathrm{O}$. The combined organic phases were washed with brine, and then dried with anhydrous sodium sulfate, filtered and concentrated. The crude reaction mixture was purified initially via gradient column chromatography on silica gel eluting with EtOAc/hexanes. Analytical purity was obtained via multiple HPLC purifications $(10 \%, 1.0 \mathrm{~g})$.

Colorless oil. ${ }^{1} \mathrm{H}$ NMR $\left(400 \mathrm{MHz}, \mathrm{CDCl}_{3}\right): \delta 2.40-2.34(\mathrm{~m}, 1 \mathrm{H}), 2.31-2.23(\mathrm{~m}, 1 \mathrm{H}), 2.19-2.14(\mathrm{~m}$, $1 \mathrm{H}), 2.13-2.06(\mathrm{~m}, 1 \mathrm{H}), 2.04-1.98(\mathrm{~m}, 1 \mathrm{H}), 1.88-1.81(\mathrm{~m}, 1 \mathrm{H}), 1.73-1.59(\mathrm{~m}, 2 \mathrm{H}), 1.42-1.32(\mathrm{~m}$, $1 \mathrm{H}) .{ }^{13} \mathrm{C}\left\{{ }^{1} \mathrm{H}\right\} \mathrm{NMR}\left(100 \mathrm{MHz}, \mathrm{CDCl}_{3}\right): \delta 213.0,51.8,41.7,33.1,27.8,24.6,21.2(\mathrm{p}, J=19.5 \mathrm{~Hz}$, 1C), 10.3 (hpt, $J=19.1 \mathrm{~Hz}, 1 \mathrm{C}$ ). HRMS (ESI-Ion Trap) m/z: [M + H]+ calcd for 132.1437 , found 132.1431 .

Diphenylethanedione- ${ }^{13} \mathrm{C}_{2}$<smiles>O=[14C](O)c1ccccc1</smiles>

1. Ethanedioyl dichloride (2.0 eq.)

2. $\mathrm{Sml}_{2}$ (2.0 eq.), THF<smiles></smiles>

To a flame-dried round-bottom flask equipped with a stir bar was added benzoic acid ( $0.50 \mathrm{~g}, 4.1$ mol), DMF (1 drop), and DCM (10 mL) under $\mathrm{N}_{2}$. Ethanedioyl dichloride $(0.70 \mathrm{~mL}, 8.1 \mathrm{mmol})$ was added dropwise while stirring at $0{ }^{\circ} \mathrm{C}$. After addition, the reaction mixture was stirred overnight and let gradually warm to rt. The reaction mixture was then concentrated, and the crude ( 4.1 mmol) dissolved in THF $(2.0 \mathrm{~mL})$. Then, $81.2 \mathrm{~mL}$ of freshly made $0.1 \mathrm{M}$ samarium (II) iodide in THF was added to the reaction mixture. The reaction mixture turned yellow after a few minutes. The mixture was purged with air before adding dilute $\mathrm{HCl}$ and diethyl ether. The organic layer was separated, and the aqueous layer was extracted with diethyl ether. All organic layers were washed with water and brine, dried over sodium sulfate and concentrated. Purification by recrystallization with ethanol afforded the title compound as a yellow solid (150 $\mathrm{mg}, 35 \%$ yield).

Yellow solid. ${ }^{1} \mathrm{H}$ NMR (400 MHz, $\left.\mathrm{CDCl}_{3}\right): \delta 8.00-7.96(\mathrm{~m}, 2 \mathrm{H}), 7.69-7.65(\mathrm{~m}, 1 \mathrm{H}), 7.55-7.50(\mathrm{~m}$, $2 \mathrm{H}) .{ }^{13} \mathrm{C}\left\{{ }^{1} \mathrm{H}\right\} \mathrm{NMR}\left(100 \mathrm{MHz}, \mathrm{CDCl}_{3}\right): \delta 194.5,134.9,132.9(\mathrm{t}, J=34.2 \mathrm{~Hz}, 1 \mathrm{C}), 129.9(\mathrm{t}, J=$ $1.9 \mathrm{~Hz}, 1 \mathrm{C}), 129.0$ (t, $J=2.2 \mathrm{~Hz}, 1 \mathrm{C}$ ). HRMS (ESI-Ion Trap) m/z: [M + H]+ calcd for 213.0826, found 213.0819 . 
1-(2,4-difluorobenzyl)-4-fluoro-1,4-diazabicyclo[2.2.2]octane-1,4-ditrifluoromethanesulfonate (compound 13-b)

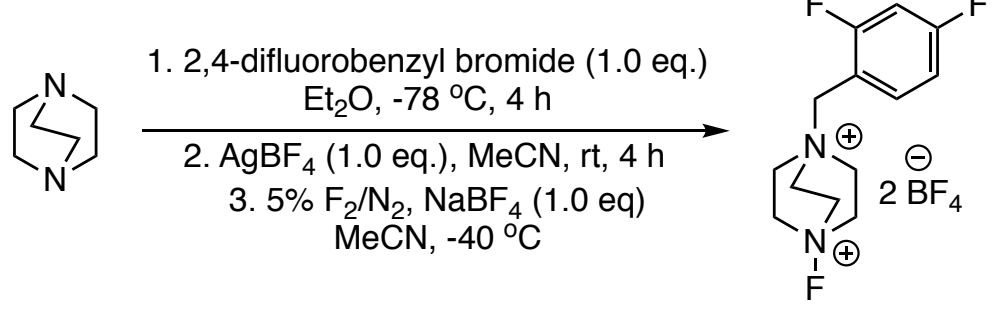

DABCO (1.3 g, $12 \mathrm{mmol})$ dissolved in $20 \mathrm{~mL}$ EtOAc, was added to a solution of 2,4difluorobenzyl bromide $(1.6 \mathrm{~mL}, 13 \mathrm{mmol})$ in $20 \mathrm{~mL}$ EtOAc and stirred for $20 \mathrm{~h}$. The salt was isolated by filtration, thoroughly washed with ether and dried in vacuo. The aforementioned crude bromide salt $(3.5 \mathrm{~g}, 10 \mathrm{mmol})$ was dissolved in $75 \mathrm{~mL} \mathrm{MeCN}$ followed by the addition of an equimolar amount of silver tetrafluoroborate and stirred for $4 \mathrm{~h}$. The precipitate of silver halide was removed by filtration. The filtrate was concentrated in vacuo and recrystallized with $\mathrm{Et}_{2} \mathrm{O} / \mathrm{MeCN}$ to afford a white solid in $81 \%$ over 2 steps $(3.2 \mathrm{~g})$.

A solution of tetrafluoroborate salt $(2.9 \mathrm{~g}, 8.9 \mathrm{mmol})$ and sodium tetrafluoroborate $(0.97 \mathrm{~g}, 8.9$ mmol) in acetonitrile $(0.02 \mathrm{M})$ was prepared in a small PTFE reactor. The mixture was purged with argon and cooled to $-40{ }^{\circ} \mathrm{C}$. Elemental $\mathrm{F}_{2}$ as a homogenous $1: 19(\mathrm{v} / \mathrm{v})$ mixture with $\mathrm{N}_{2}$ was introduced at a flow rate of approx. $10 \mathrm{~mL} / \mathrm{min}$ into the rapidly stirred mixture via PTFE tubing at $-40{ }^{\circ} \mathrm{C}$ (Caution: Poison, corrosive, toxic). The mixture was purged with argon and warmed to room temperature before filtration to remove sodium fluoride, and the solution was evaporated under reduced pressure. Purification by recrystallization with $\mathrm{Et}_{2} \mathrm{O} / \mathrm{MeCN}$ afforded the title compound as a white solid (2.9 g, $75 \%$ yield).

White solid. ${ }^{1} \mathrm{H}$ NMR (400 MHz, $\left.\mathrm{CDCl}_{3}\right): \delta$ 7.69-7.63 (m, 1H), 7.26-7.20 (m, 2H), $4.82(\mathrm{~s}, 2 \mathrm{H})$, 4.72-4.67 (m, 6H), 4.27-4.23 (m, 6H). ${ }^{13} \mathrm{C}\left\{{ }^{1} \mathrm{H}\right\}$ NMR $\left(100 \mathrm{MHz}, \mathrm{CDCl}_{3}\right): \delta 167.8,167.7$, $165.5,165.2$, 165.1, 165.0, 162.6,162.5, 137.73,137.69, 137.62,137.58, 114.39,114.35, 114.2, $114.1,110.52,110.48,110.4,110.3,106.5,106.2,106.0$. 19F NMR (282 $\left.\mathrm{MHz}, \mathrm{CDCl}_{3}\right): \delta 48.9$ (bs), -104.2 - (-104.3) (m), -108.4 - (-108.5) (m), -150.6 - (-150.7) (m). HRMS (ESI-Ion Trap) $\mathrm{m} / \mathrm{z}$ : calcd for 258.1332, found 258.1294 (observed without $\mathrm{BF}_{4}$ counter ions; also, rapid decomposition gave rise to a large peak corresponding to a defluorinated species at 239.1355). 
2'-fluoro-[1,1'-biphenyl]-2-carbonyl fluoride (compound 7)

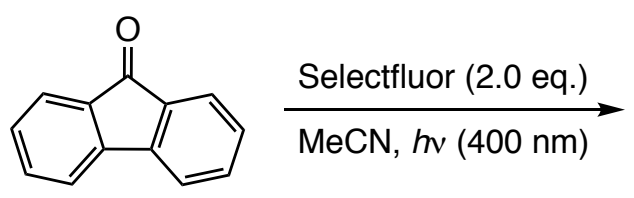<smiles>O=C(F)c1ccccc1-c1ccccc1F</smiles>

Selectfluor $(97 \mathrm{mg}, 0.28 \mathrm{mmol})$ and 9-fluorenone $(0.13 \mathrm{mmol})$ were added to an oven-dried $\mu \omega$ vial equipped with a stir bar. The vial was then sealed with a cap with septum using a crimper and evacuated/refilled with $\mathrm{N}_{2}$ multiple times. Anhydrous $\mathrm{CH}_{3} \mathrm{CN}(6 \mathrm{~mL})$ was added, and the reaction mixture was irradiated at $400 \mathrm{~nm}$ with LEDs overnight while stirring. Upon completion, a $0.3 \mathrm{~mL}$ aliquot was taken for ${ }^{19} \mathrm{~F}$ NMR yield determination, and the rest of the reaction mixture was poured over $\mathrm{Et}_{2} \mathrm{O}$, filtered through Celite and concentrated. The crude reaction mixture was purified initially via gradient column chromatography on silica gel eluting with EtOAc/hexanes. Analytical purity was obtained via recrystallization with hexanes.

White solid. ${ }^{1} \mathrm{H}$ NMR (400 MHz, $\mathrm{CDCl}_{3}$ ): $\delta 8.10(\mathrm{dd}, J=7.9,1.4 \mathrm{~Hz}, 1 \mathrm{H}), 7.73(\mathrm{td}, J=7.6,1.4$ $\mathrm{Hz}, 1 \mathrm{H}), 7.55(\mathrm{tt}, J=7.6,1.0 \mathrm{~Hz}, 1 \mathrm{H}), 7.45-7.37(\mathrm{~m}, 2 \mathrm{H}), 7.33(\mathrm{dm}, J=2.0 \mathrm{~Hz}, 1 \mathrm{H}), 7.31(\mathrm{dm}, J$ $=2.0 \mathrm{~Hz}, 1 \mathrm{H}), 7.29(\mathrm{dm}, J=2.0 \mathrm{~Hz}, 1 \mathrm{H}), 7.26-7.22(\mathrm{~m}, 1 \mathrm{H}), 7.16-7.11(\mathrm{~m}, 1 \mathrm{H}) .{ }^{13} \mathrm{C}\left\{{ }^{1} \mathrm{H}\right\} \mathrm{NMR}$ $\left(100 \mathrm{MHz}, \mathrm{CDCl}_{3}\right): \delta 160.72,160.71,158.5,158.28,158.26,155.1,139.13,139.09,134.5$, $132.32,132.30,132.22,132.19,130.5,130.4,130.2,130.1,128.5,128.0,127.8,125.22,125.21$, 124.64, 124.63, 124.4, 124.3, 115.6, 115.4. ${ }^{19} \mathrm{~F} \mathrm{NMR}\left(282 \mathrm{MHz}, \mathrm{CDCl}_{3}\right): \delta 29.8,-115.7-(-115.8)$ (m).

$(3 S, 8 R, 9 S, 10 R, 13 S, 14 S, 17 S)$-10,13-dimethyl-7-oxo-2,3,4,7,8,9,10,11,12,13,14,15,16,17tetradecahydro- $1 H$-cyclopenta $[a]$ phenanthrene-3,17-diyl-15- $d$ diacetate (compound 17)

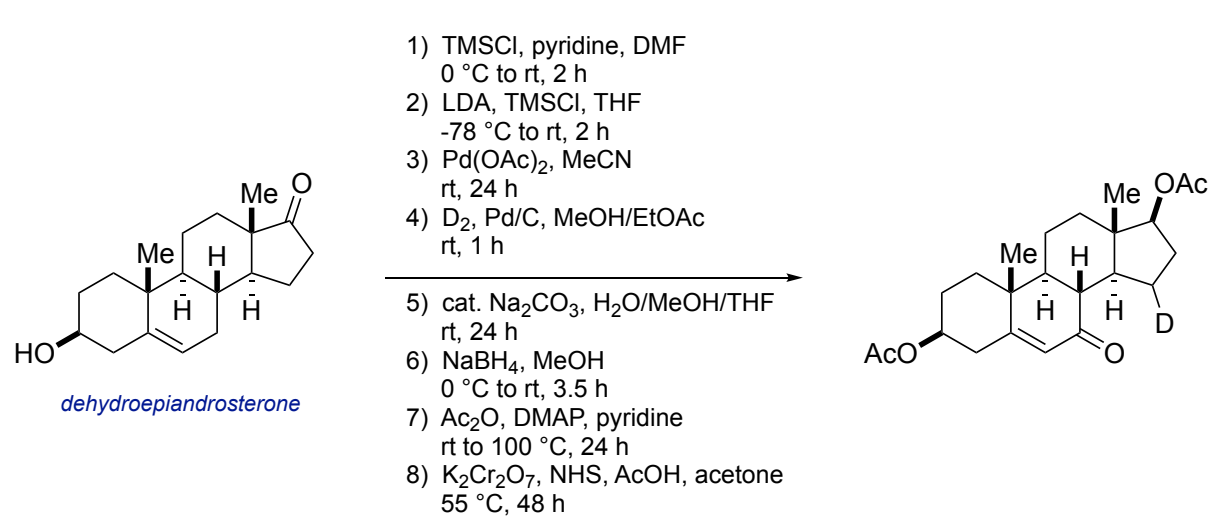

Dehydroepiandrosterone ( $3.0 \mathrm{~g}, 10.4 \mathrm{mmol}, 1.0$ equiv.) was dissolved in a mixture of DMF (28 $\mathrm{mL}$ ) and pyridine $(12 \mathrm{~mL})$. The mixture was cooled to $0{ }^{\circ} \mathrm{C}$ in an ice bath and stirred under $\mathrm{N}_{2}$, then TMSCl (3.3 mL, $26 \mathrm{mmol}, 2.5$ equiv.) was added dropwise. The mixture stirred at $0{ }^{\circ} \mathrm{C}$ for 10 minutes and then was allowed to warm to room temperature and stir for $2 \mathrm{~h}$. Water $(150 \mathrm{~mL})$ was added to quench the reaction, and the resulting mixture was extracted with $\mathrm{Et}_{2} \mathrm{O}(50 \mathrm{~mL} \times 3)$. The combined ether extracts were washed with $\mathrm{H}_{2} \mathrm{O}(150 \mathrm{~mL} \times 2)$, brine $(100 \mathrm{~mL})$ and then were 
dried over $\mathrm{Na}_{2} \mathrm{SO}_{4}$. The solvent was removed in vacuo to provide TMS-protected DHEA in quantitative yield, which was used without further purification in the next step.

The TMS-protected DHEA (1.71 g, $4.75 \mathrm{mmol}, 1.0$ equiv) was dissolved in anhydrous THF $(10 \mathrm{~mL})$ and added dropwise to a solution of freshly prepared LDA in $10 \mathrm{~mL}$ THF $(9.975 \mathrm{mmol}$, 2.1 equiv.) at $-78{ }^{\circ} \mathrm{C}$ under $\mathrm{N}_{2}$. The solution stirred at $-78^{\circ} \mathrm{C}$ for $35 \mathrm{~min}$ to which TMSCl (1.22 $\mathrm{mL}, 9.5 \mathrm{mmol}, 2.0$ equiv.) was added dropwise. The reaction stirred for a few minutes at $-78{ }^{\circ} \mathrm{C}$, then slowly warmed up to room temperature and stirred for $1.5 \mathrm{~h}$. The solution was carefully quenched with brine $(100 \mathrm{~mL})$ then extracted with EtOAc $(50 \mathrm{~mL}$ x 3). The combined organic layers were then washed with sat. sodium bicarbonate $(50 \mathrm{~mL})$ followed with brine $(100 \mathrm{~mL})$. The solvent was dried over $\mathrm{Na}_{2} \mathrm{SO}_{4}$ and then concentrated to dryness to provide the silyl enol ether of the TMS-protected DHEA in quantitative yield, which was used without further purification in the next step.

The material from the previous step ( $1.93 \mathrm{~g}, 4.45 \mathrm{mmol}, 1.0$ equiv.) was dissolved in anhydrous $\mathrm{MeCN}(70 \mathrm{~mL})$ and stirred under $\mathrm{N}_{2} . \mathrm{Pd}(\mathrm{OAc})_{2}(1.0 \mathrm{~g}, 4.67 \mathrm{mmol}, 1.05$ equiv.) was added in one portion and the mixture stirred overnight at room temperature. The mixture was diluted with $\mathrm{Et}_{2} \mathrm{O}$ $(50 \mathrm{~mL})$ and filtered through a small silica plug, which was then washed with DCM. The combined solvents were concentrated to dryness, in vacuo, to provide the enone in quantitative yield, which was used without further purification in the next step.

The enone from the previous step (1.58 g, $4.4 \mathrm{mmol}, 1.0$ equiv.) was dissolved in $60 \mathrm{~mL}$ of a 7:1 mixture of $\mathrm{MeOH}:$ EtOAc in a round-bottom flask. After degassing the mixture with $\mathrm{N}_{2}, \mathrm{Pd} / \mathrm{C}$ (100 mg, 0.02 equiv.) was carefully added in one portion. Deuterium gas (Cambridge Isotope Laboratories, Inc.) was introduced into the flask via balloon, after briefly placing the mixture under vacuum. The mixture stirred vigorously under $\mathrm{D}_{2}$ for $1 \mathrm{~h}$, at which time a crude NMR aliquot determined that the D-ring enone had been fully deuterated, while the B-ring olefin remained intact. The mixture was filtered through a short plug of Celite and concentrated to dryness, providing the desired di-deuterated intermediate in quantitative yield, which was used without further purification in the next step.

The di-deutero ketone from the previous step (1.60 g, $4.4 \mathrm{mmol}, 1.0$ equiv.) was dissolved in $20 \mathrm{~mL}$ of $1: 1 \mathrm{THF} / \mathrm{MeOH}$ and catalytic $\mathrm{Na}_{2} \mathrm{CO}_{3}(17 \mathrm{mg}, 0.035$ equiv.) was added in $10 \mathrm{~mL}$ of DI water. The mixture was stirred vigorously for $24 \mathrm{~h}$ at room temperature. After quenching with $2 \mathrm{M}$ $\mathrm{HCl}(6 \mathrm{~mL})$, the mixture stirred for $30 \mathrm{~min}$ then most of the solvent was removed in vacuo. The mixture was extracted with DCM $(50 \mathrm{~mL} \times 3)$, then the combined organic layers were washed with brine and dried over $\mathrm{Na}_{2} \mathrm{SO}_{4}$. The solution was concentrated to dryness in vacuo, providing the mono-deutero intermediate in quantitative yield, which was used without further purification in the next step.

The material from the previous step ( $1.27 \mathrm{~g}, 4.4 \mathrm{mmol}, 1.0$ equiv.) was dissolved in $\mathrm{MeOH}(35$ $\mathrm{mL}$ ) and cooled to $0{ }^{\circ} \mathrm{C}$ in an ice bath and stirred under $\mathrm{N}_{2} . \mathrm{NaBH}_{4}(218 \mathrm{mg}, 5.5 \mathrm{mmol}, 1.25$ equiv.) was carefully added in three portions; the mixture stirred at $0{ }^{\circ} \mathrm{C}$ for $30 \mathrm{~min}$ and then slowly warmed up to room temperature and stirred for $3 \mathrm{~h}$. The mixture was concentrated to dryness and 
used crude in the subsequent step to avoid a difficult isolation of the insoluble product via an aqueous workup (quantitative yield).

The diol prepared in the previous step (1.28 g, $4.4 \mathrm{mmol}, 1.0$ equiv.) was dissolved in pyridine $(8 \mathrm{~mL})$ and $\mathrm{MeCN}(8 \mathrm{~mL})$ and catalytic DMAP $(25 \mathrm{mg})$ was added. The mixture was stirred under $\mathrm{N}_{2}$ and cooled to $0{ }^{\circ} \mathrm{C}$ in an ice bath; $\mathrm{Ac}_{2} \mathrm{O}(6 \mathrm{~mL})$ was then added dropwise. The mixture slowly warmed up to room temperature and stirred for $1 \mathrm{~h}$ then was heated at $100{ }^{\circ} \mathrm{C}$ for $12 \mathrm{~h}$. After concentrating down the solvent in vacuo, the mixture was quenched with $\mathrm{H}_{2} \mathrm{O}(150 \mathrm{~mL})$, and then extracted with $\mathrm{Et}_{2} \mathrm{O}(50 \mathrm{~mL} \times 3)$. The combined organic extracts were successively washed with 100-150 mL of $\mathrm{H}_{2} \mathrm{O}, 1 \mathrm{M} \mathrm{HCl}$, sat. sodium bicarbonate and brine then were dried over $\mathrm{Na}_{2} \mathrm{SO}_{4}$ and concentrated to dryness - providing the diacetylated product in quantitative yield, which was used without further purification in the next step.

The material from the previous step ( $1.65 \mathrm{~g}, 4.4 \mathrm{mmol}, 1.0$ equiv.) was dissolved in acetone (75 $\mathrm{mL}$ ) and stirred in a round-bottom flask under $\mathrm{N}_{2} . \mathrm{K}_{2} \mathrm{Cr}_{2} \mathrm{O}_{7}(5.4 \mathrm{~g}, 17.6 \mathrm{mmol}, 4.0$ equiv.) and $N$-hydroxysuccinimide (1.6 g, $13.2 \mathrm{mmol}, 3.0$ equiv.) were added in one portion followed by glacial acetic acid $(7.5 \mathrm{~mL})$. The mixture was heated at $55^{\circ} \mathrm{C}$ for $24 \mathrm{~h}$. An additional $1.5 \mathrm{~g} \mathrm{~K}_{2} \mathrm{Cr}_{2} \mathrm{O}_{7}$ and $0.5 \mathrm{~g}$ NHS was then added and the mixture was heated overnight at the same temperature. The mixture was cooled to room temperature, diluted with $\mathrm{Et}_{2} \mathrm{O}$ and filtered through a small silica plug, which was then washed with DCM. The combined organic solvents were concentrated under vacuo to remove most of the acetone then diluted with $\mathrm{H}_{2} \mathrm{O}(150 \mathrm{~mL})$. The water layer was extracted with DCM (50 mL x 3), and the combined organic layers were then successively washed with sat. sodium bicarbonate and brine then dried over $\mathrm{Na}_{2} \mathrm{SO}_{4}$. The solvent was removed in vacuo to provide crude deuterium labeled steroid 19, which was recrystallized from $\mathrm{MeOH}$ to give pure material.

White solid (1.6:1.0 isotopomeric mixture). ${ }^{1} \mathrm{H} \mathrm{NMR}\left(400 \mathrm{MHz}, \mathrm{CDCl}_{3}\right): \delta 5.71(\mathrm{~d}, J=1.8 \mathrm{~Hz}$, $1 \mathrm{H}), 4.75-4.67(\mathrm{~m}, 1 \mathrm{H}), 4.64-4.61(\mathrm{~m}, 1 \mathrm{H}), 2.59-2.54(\mathrm{~m}, 1 \mathrm{H}), 2.50-2.39(\mathrm{~m}, 1.7 \mathrm{H}), 2.27(\mathrm{t}, J=$ $10.9 \mathrm{~Hz}, 1 \mathrm{H}), 2.17(\mathrm{t}, J=9.2 \mathrm{~Hz}, 0.7 \mathrm{H}), 2.05(\mathrm{~s}, 3 \mathrm{H}), 2.04(\mathrm{~s}, 3 \mathrm{H}), 2.02-1.94(\mathrm{~m}, 2.7 \mathrm{H}), 1.78-1.73$ $(\mathrm{m}, 1 \mathrm{H}), 1.70-1.62(\mathrm{~m}, 1.7 \mathrm{H}), 1.58-1.46(\mathrm{~m}, 3 \mathrm{H}), 1.44-1.36(\mathrm{~m}, 1 \mathrm{H}), 1.31-1.24(\mathrm{~m}, 1 \mathrm{H}), 1.23-1.13$ $(\mathrm{m}, 4.6 \mathrm{H}), 0.82-0.77(\mathrm{~m}, 3 \mathrm{H}) ;{ }^{13} \mathrm{C}\left\{{ }^{1} \mathrm{H}\right\} \mathrm{NMR}(100 \mathrm{MHz}, \mathrm{CDCl} 3): \delta 201.1,171.1,170.2,164.3$, 126.5, 81.9, 72.1, 49.8, 49.7, 45.0, 44.9, 44.7, 43.1, 43.0, 38.4, 37.8, 36.0, 35.8, 27.3, 21.2, 21.1, 20.7, 17.3, 12.1, 12.0; ${ }^{2} \mathrm{H} \mathrm{NMR}\left(400 \mathrm{MHz}, \mathrm{CDCl}_{3}\right): \delta 2.25$ (d, $\left.J=12.6 \mathrm{~Hz}, 0.38 \mathrm{D}\right), 1.47$ (s, 0.62D); HRMS (ESI-FTICR-MS) m/z: [M + Na]+ calcd for 412.2210, found 412.2203. 


\section{Spectral Data:}

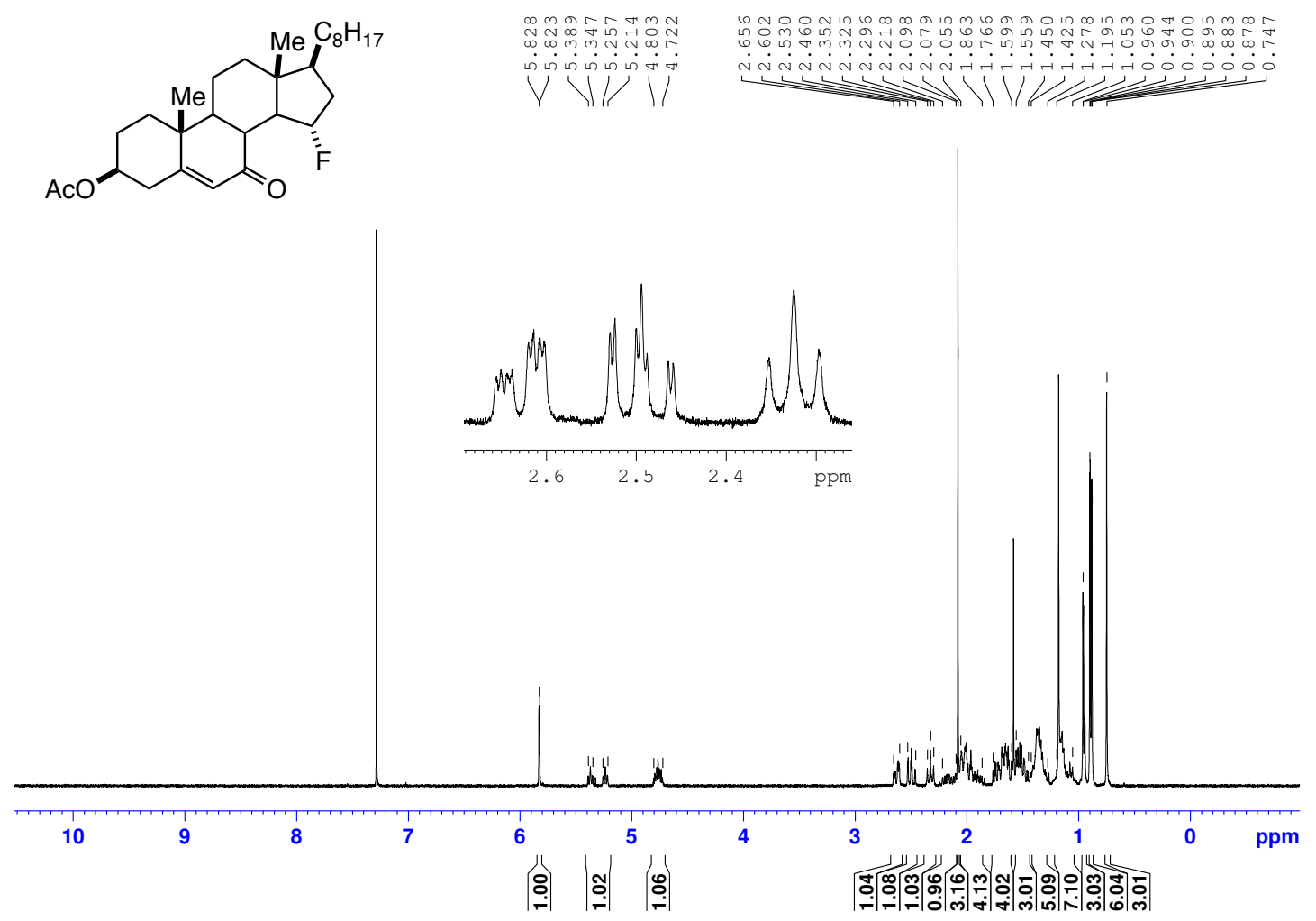

Figure S7. ${ }^{1} \mathrm{H}$ NMR spectrum of $15 \mathrm{~S}-$ fluoro-7-keto-cholesteryl acetate (major diastereomer) in $\mathrm{CDCl}_{3}$. 

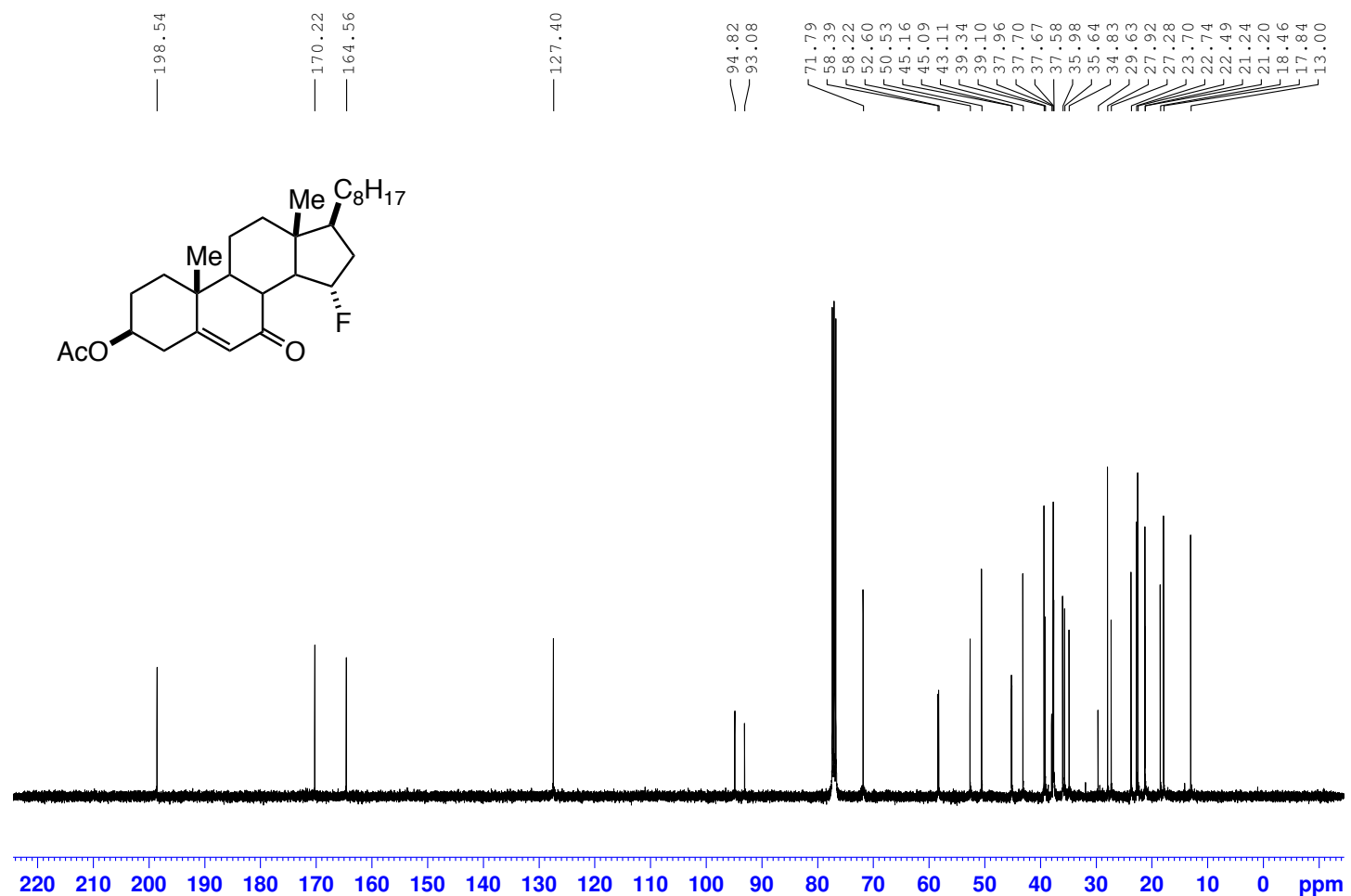

Figure S8. ${ }^{13} \mathrm{C}\left\{{ }^{1} \mathrm{H}\right\}$ NMR spectrum of $15 \mathrm{~S}$-fluoro-7-keto-cholesteryl acetate (major diastereomer) in $\mathrm{CDCl}_{3}$. 

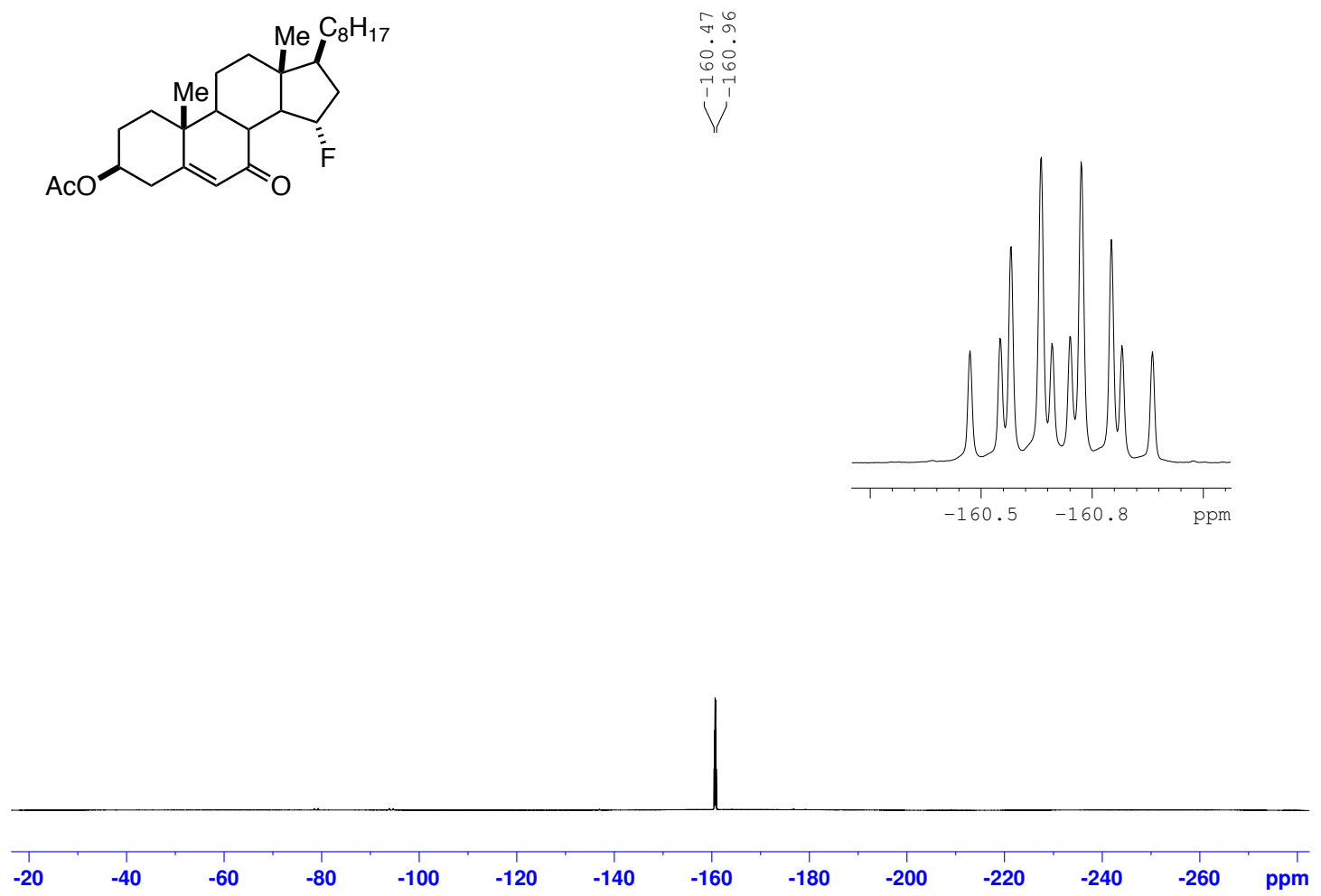

Figure S9. ${ }^{19} \mathrm{~F}$ NMR spectrum of $15 \mathrm{~S}-$-fluoro-7-keto-cholesteryl acetate (major diastereomer) in $\mathrm{CDCl}_{3}$. 


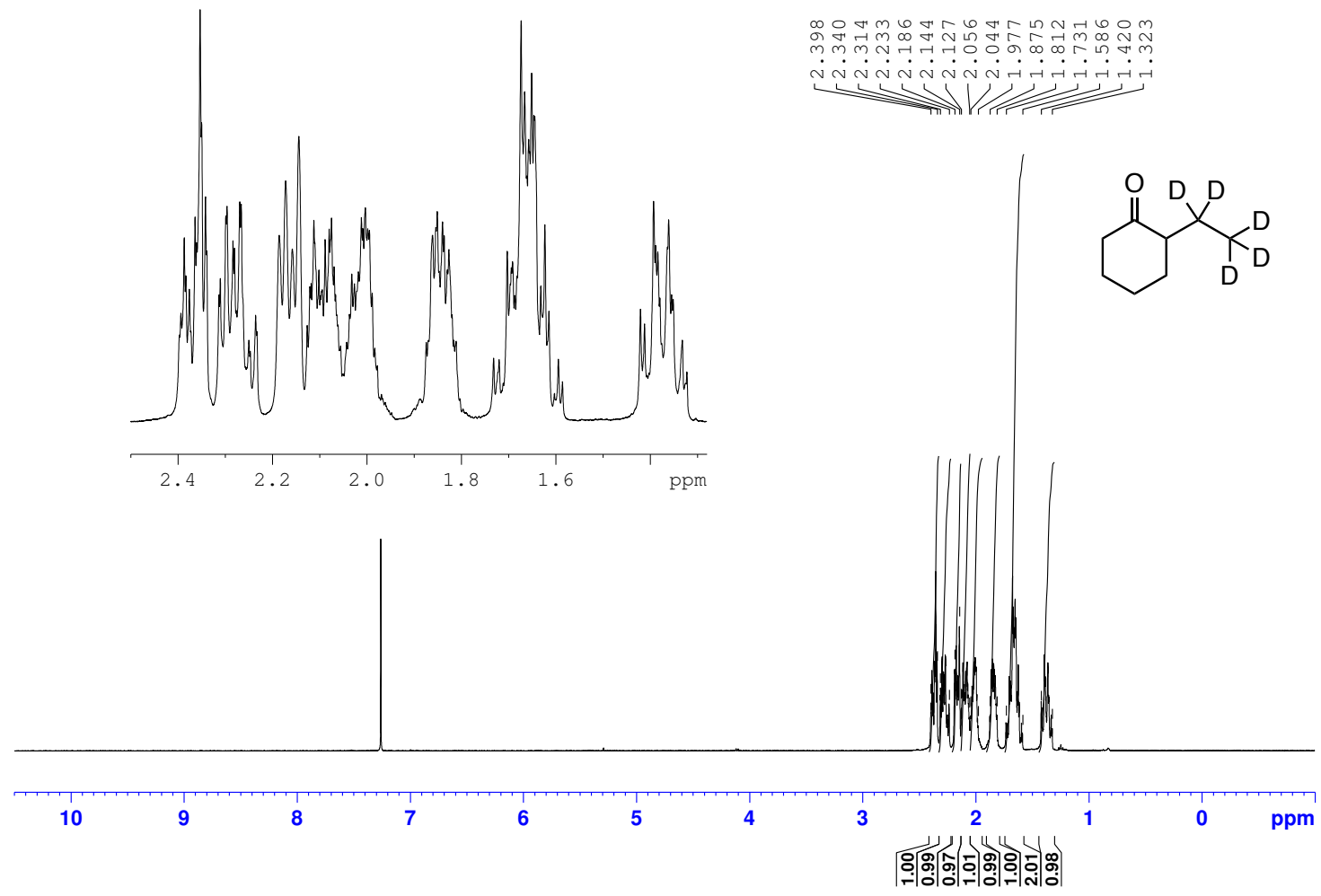

Figure S10. ${ }^{1} \mathrm{H}$ NMR spectrum of 2-ethyl-D - -cyclohexan-1-one in $\mathrm{CDCl}_{3}$. 


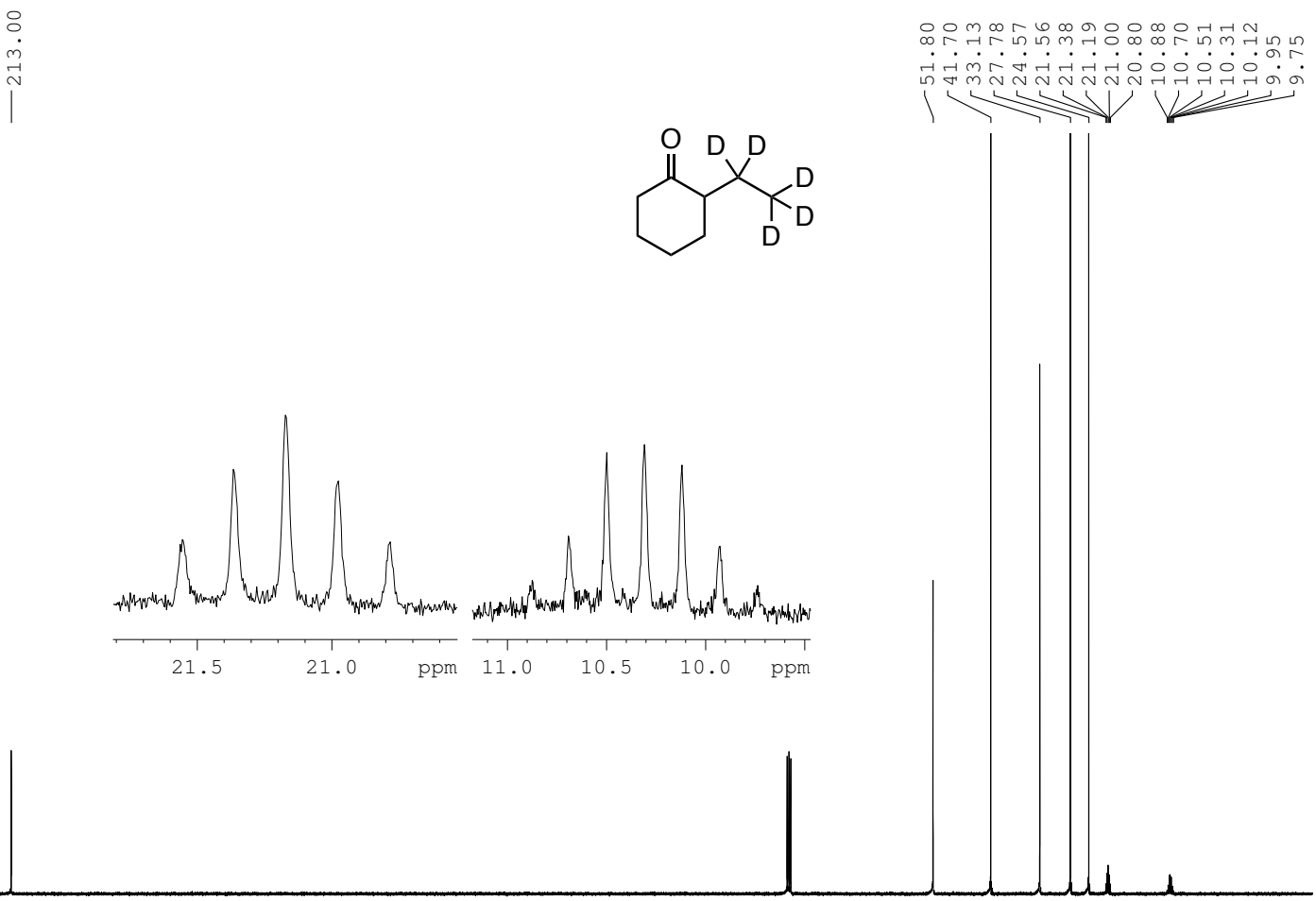

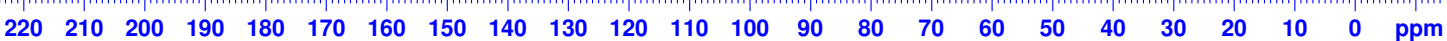

Figure S11. ${ }^{13} \mathrm{C}\left\{{ }^{1} \mathrm{H}\right\}$ NMR spectrum of 2-ethyl-D 5 cyclohexan-1-one in $\mathrm{CDCl}_{3}$. 


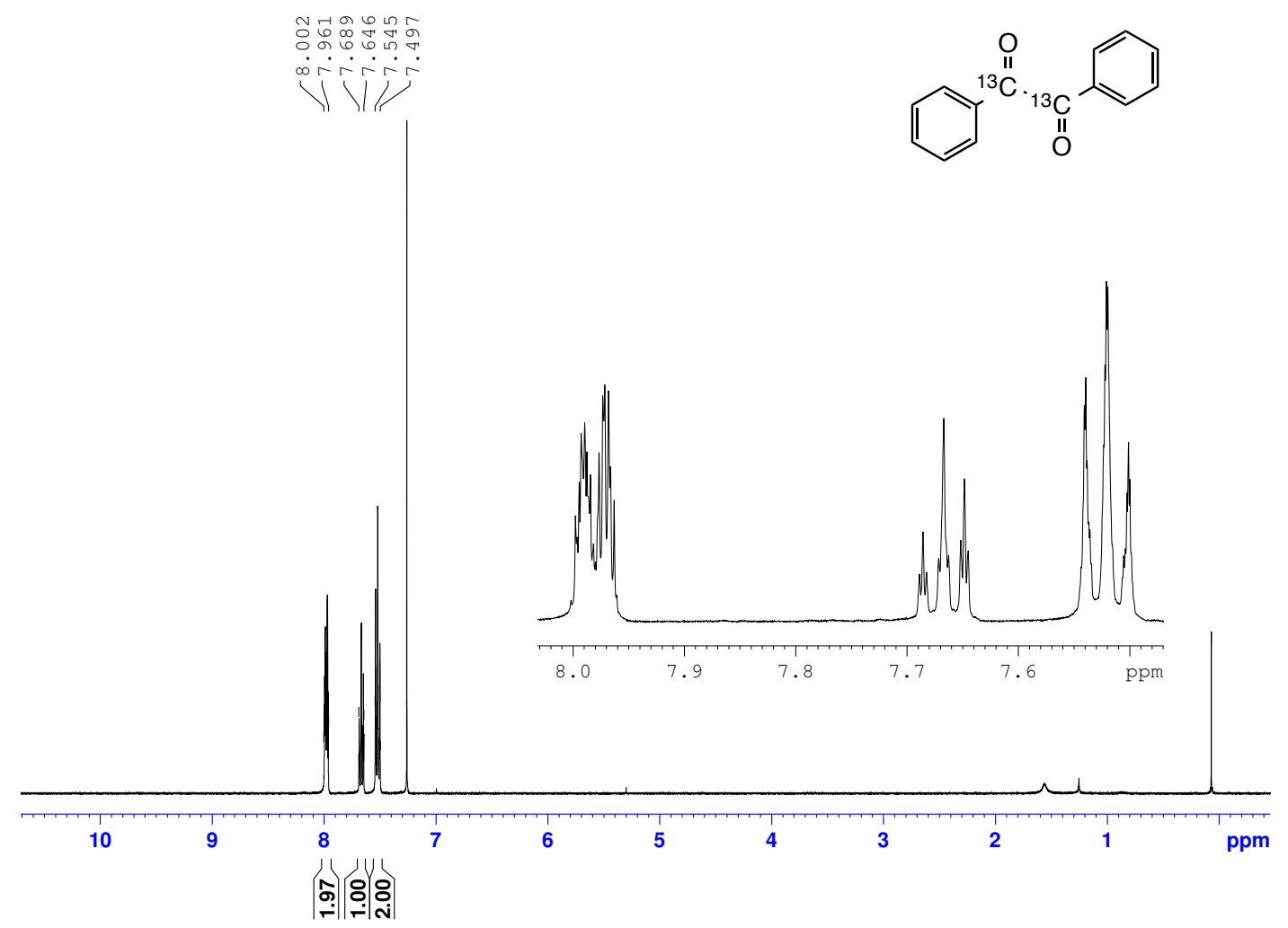

Figure S12. ${ }^{1} \mathrm{H}$ NMR spectrum of diphenylethanedione- $-{ }^{13} \mathrm{C}_{2}$ in $\mathrm{CDCl}_{3}$. 


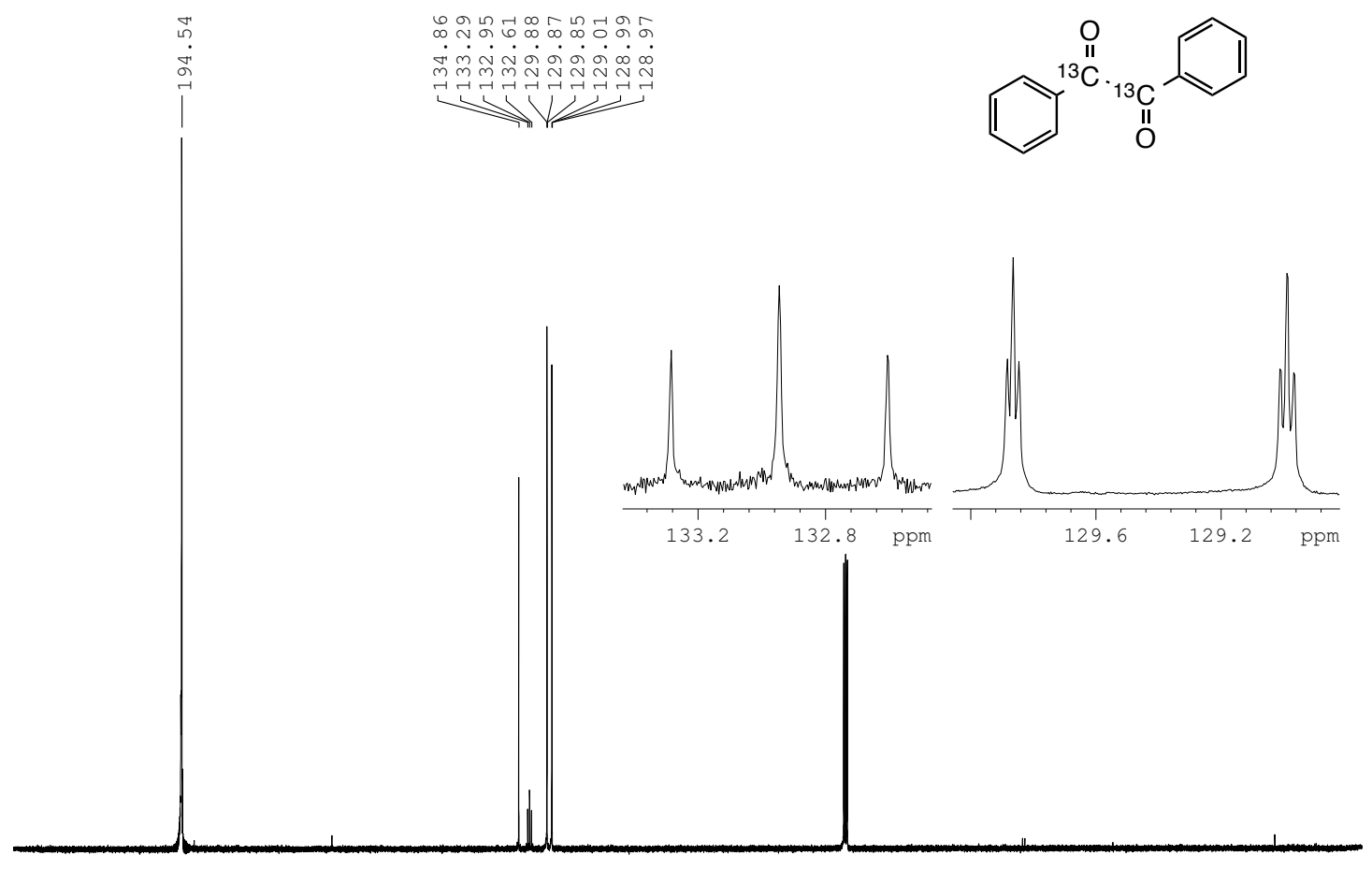

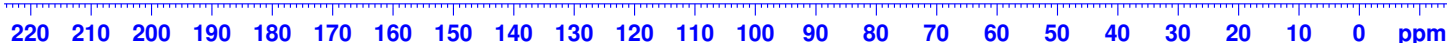

Figure S13. ${ }^{13} \mathrm{C}\left\{{ }^{1} \mathrm{H}\right\}$ NMR spectrum of diphenylethanedione- ${ }^{13} \mathrm{C}_{2}$ in $\mathrm{CDCl}_{3}$. 


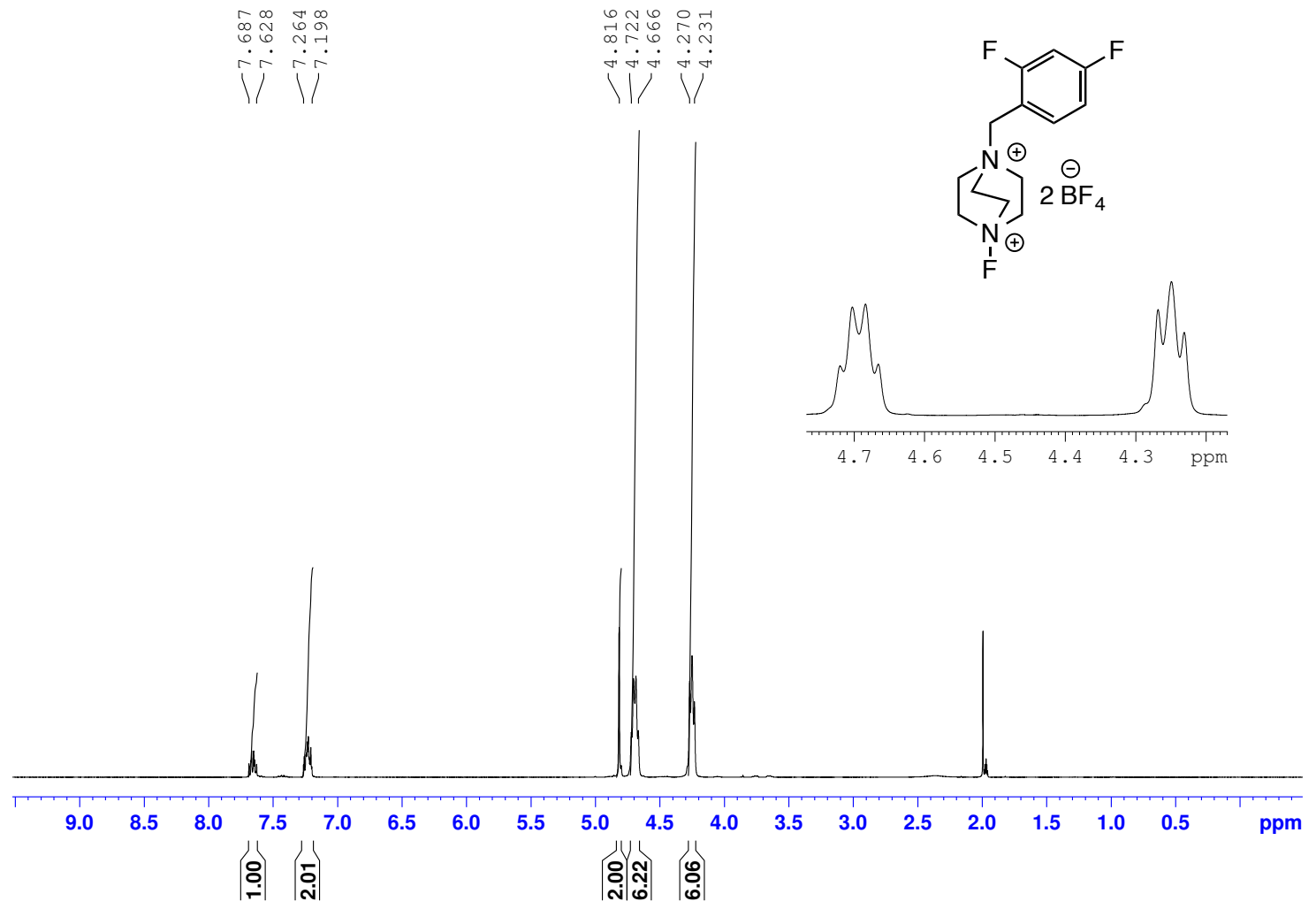

Figure S14. ${ }^{1}$ H NMR spectrum of 1-(2,4-difluorobenzyl)-4-fluoro-1,4-diazabicyclo[2.2.2] octane1,4-bis-tetrafluoroborate (compound 13-b) in $\mathrm{CD}_{3} \mathrm{CN}$. 


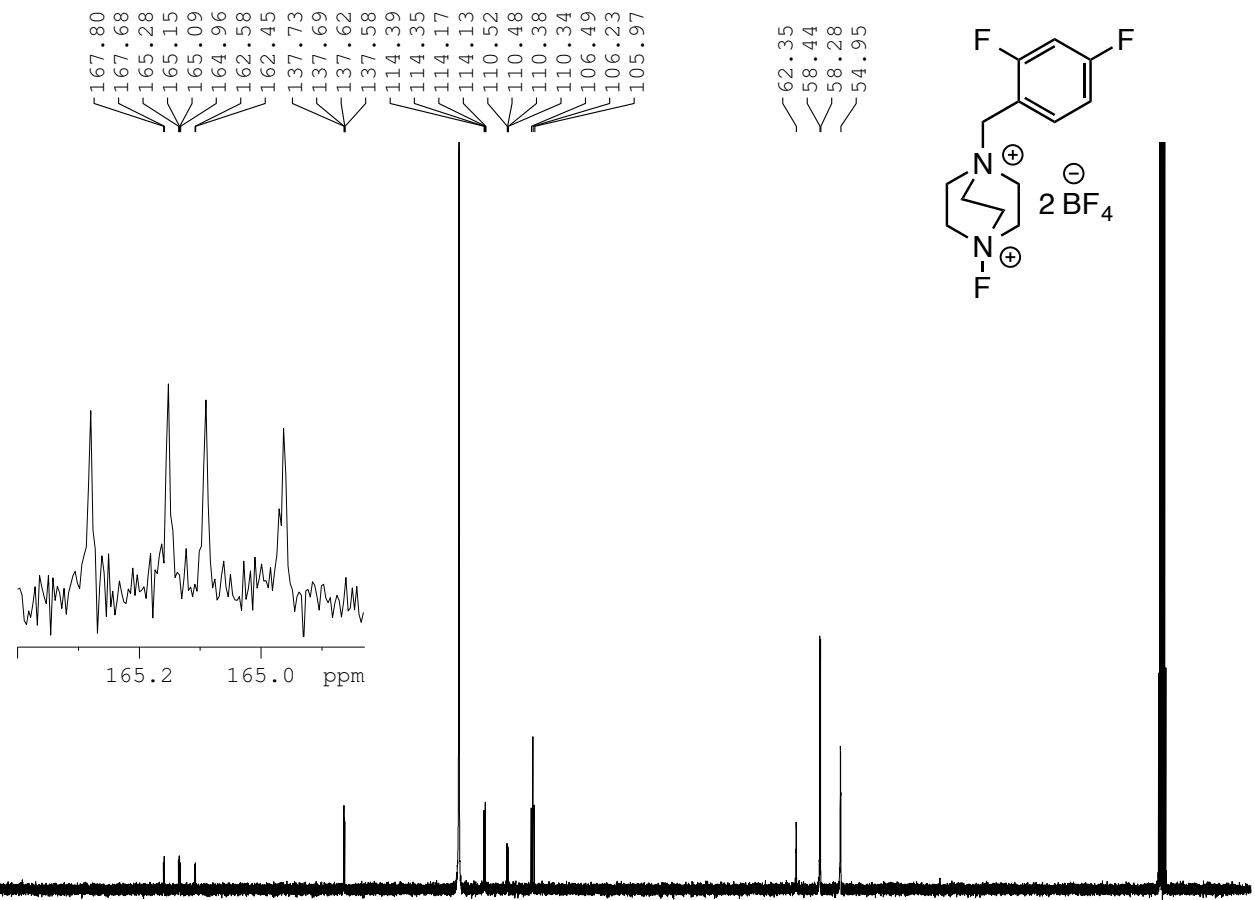

$\begin{array}{llllllllllllllllllllllll}220 & 210 & 200 & 190 & 180 & 170 & 160 & 150 & 140 & 130 & 120 & 110 & 100 & 90 & 80 & 70 & 60 & 50 & 40 & 30 & 20 & 10 & 0 & \mathrm{ppm}\end{array}$

Figure S15. ${ }^{13} \mathrm{C}\left\{{ }^{1} \mathrm{H}\right\} \quad$ NMR spectrum of 1-(2,4-difluorobenzyl)-4-fluoro-1,4diazabicyclo[2.2.2] octane-1,4-bis-tetrafluoroborate (compound 13-b) in $\mathrm{CD}_{3} \mathrm{CN}$. 


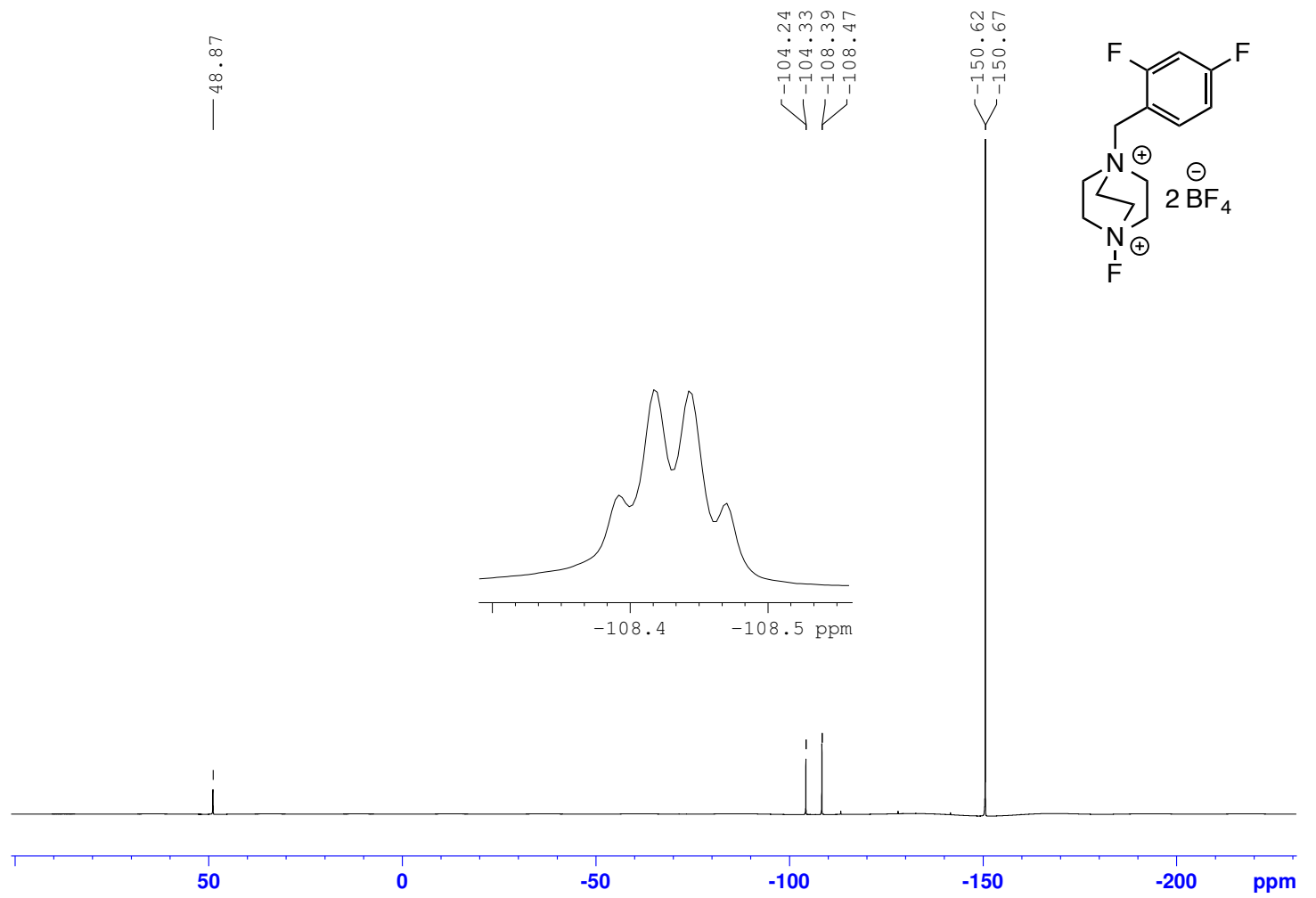

Figure S16. ${ }^{19}$ F NMR spectrum of 1-(2,4-difluorobenzyl)-4-fluoro-1,4-diazabicyclo[2.2.2] octane bis-tetrafluoroborate (compound 13-b) in $\mathrm{CD}_{3} \mathrm{CN}$. 


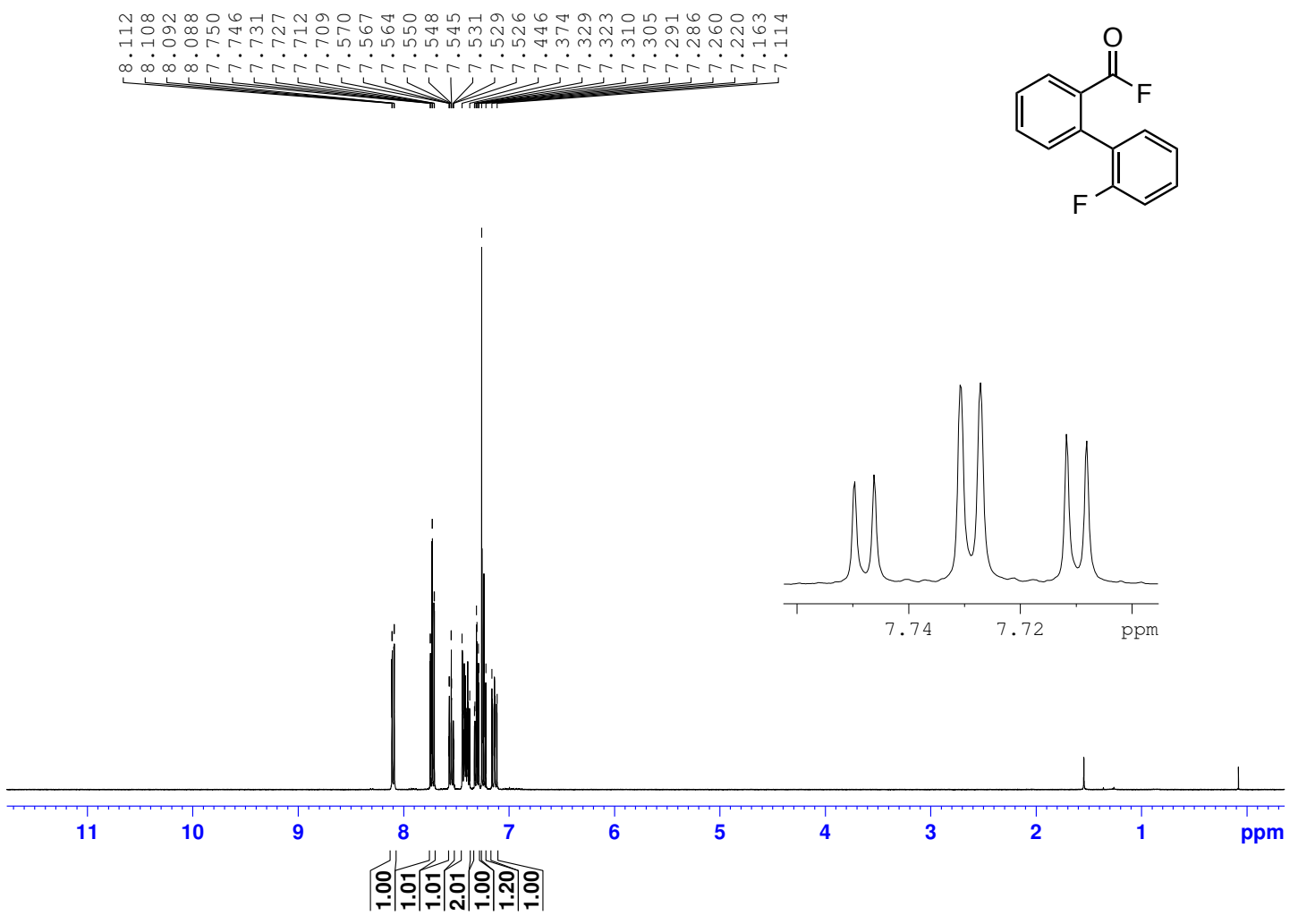

Figure S17. ${ }^{1} \mathrm{H}$ NMR spectrum of 2'-fluoro-[1,1'-biphenyl]-2-carbonyl fluoride (compound 7) in $\mathrm{CDCl}_{3}$. 


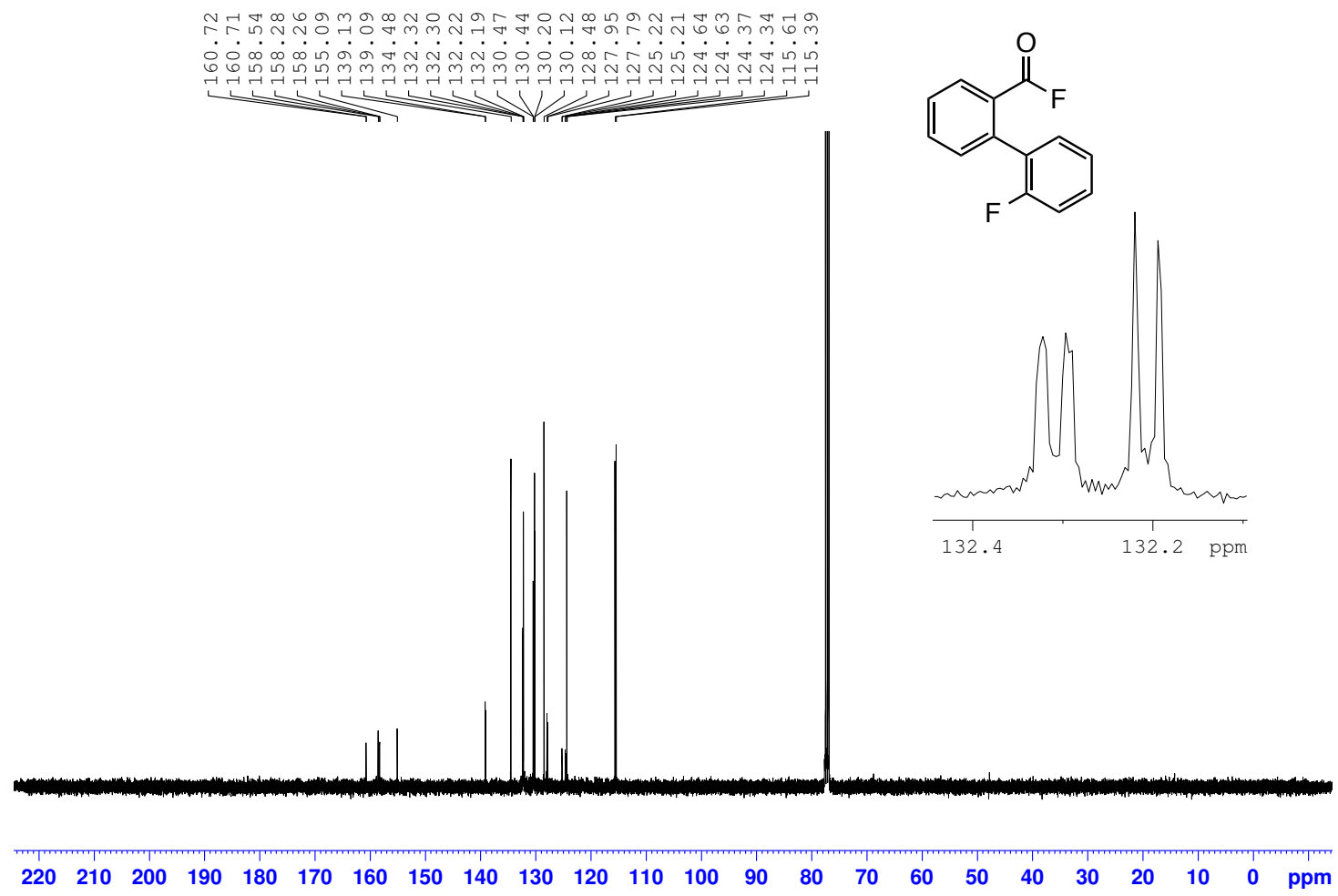

Figure S18. ${ }^{13} \mathrm{C}\left\{{ }^{1} \mathrm{H}\right\}$ NMR spectrum of 2'-fluoro-[1,1'-biphenyl]-2-carbonyl fluoride (compound 7) in $\mathrm{CDCl}_{3}$. 


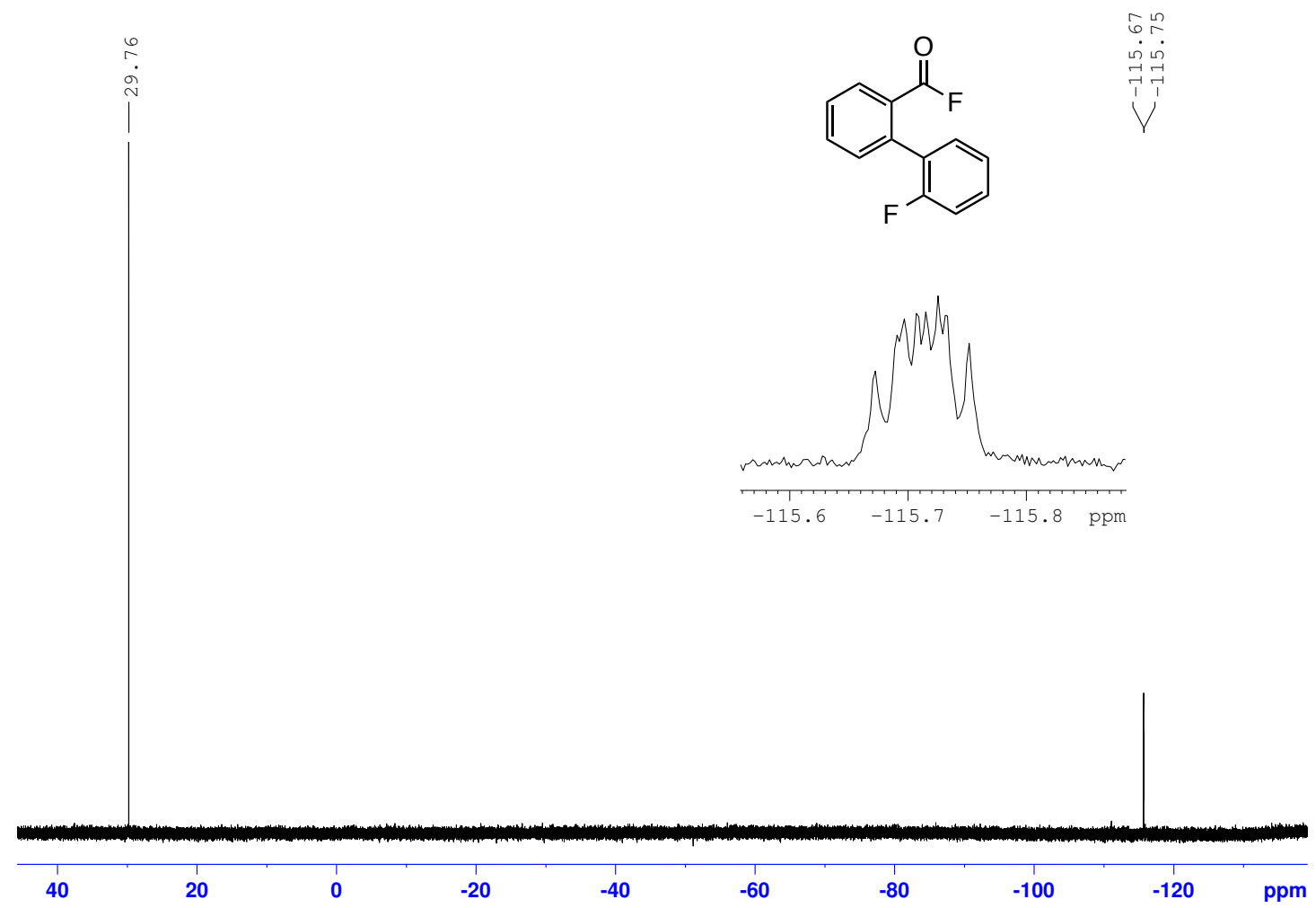

Figure S19. ${ }^{19} \mathrm{~F}$ NMR spectrum of 2'-fluoro-[1,1'-biphenyl]-2-carbonyl fluoride (compound 7) in $\mathrm{CDCl}_{3}$. 


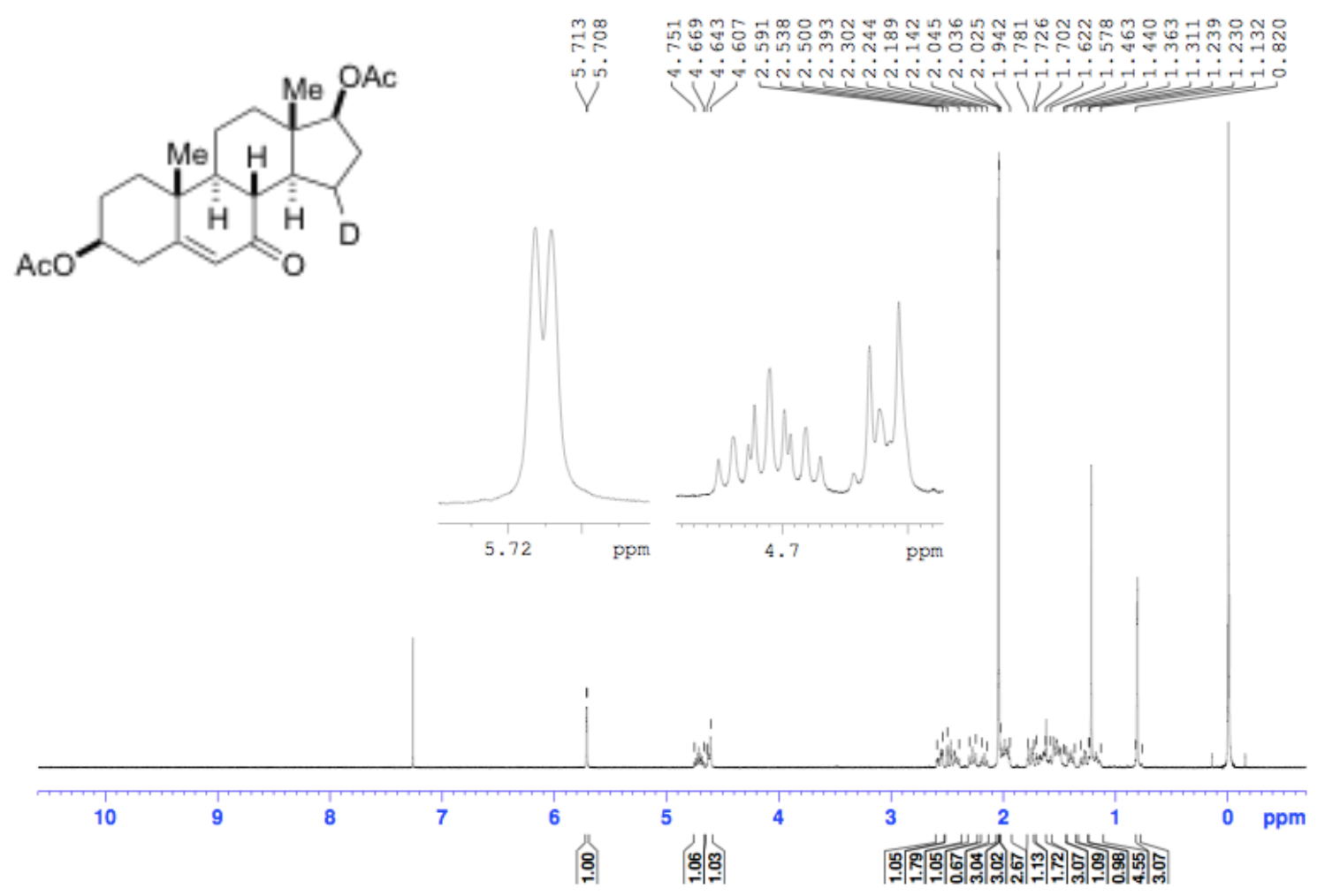

Figure S20. ${ }^{1} \mathrm{H}$ NMR spectrum of compound 17 in $\mathrm{CDCl}_{3}$. 


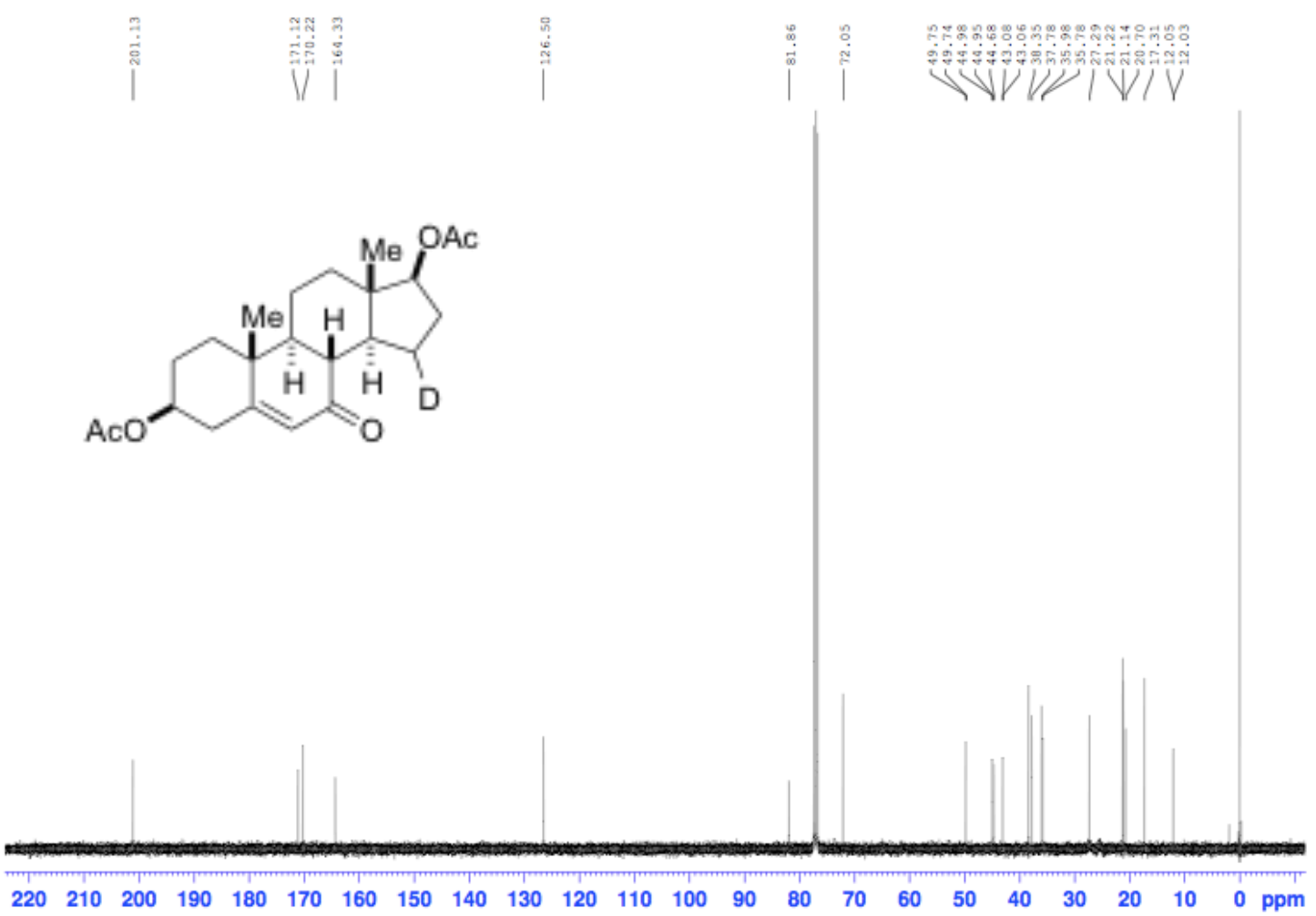

Figure S21. ${ }^{13} \mathrm{C}\left\{{ }^{1} \mathrm{H}\right\}$ NMR spectrum of compound 17 in $\mathrm{CDCl}_{3}$. 


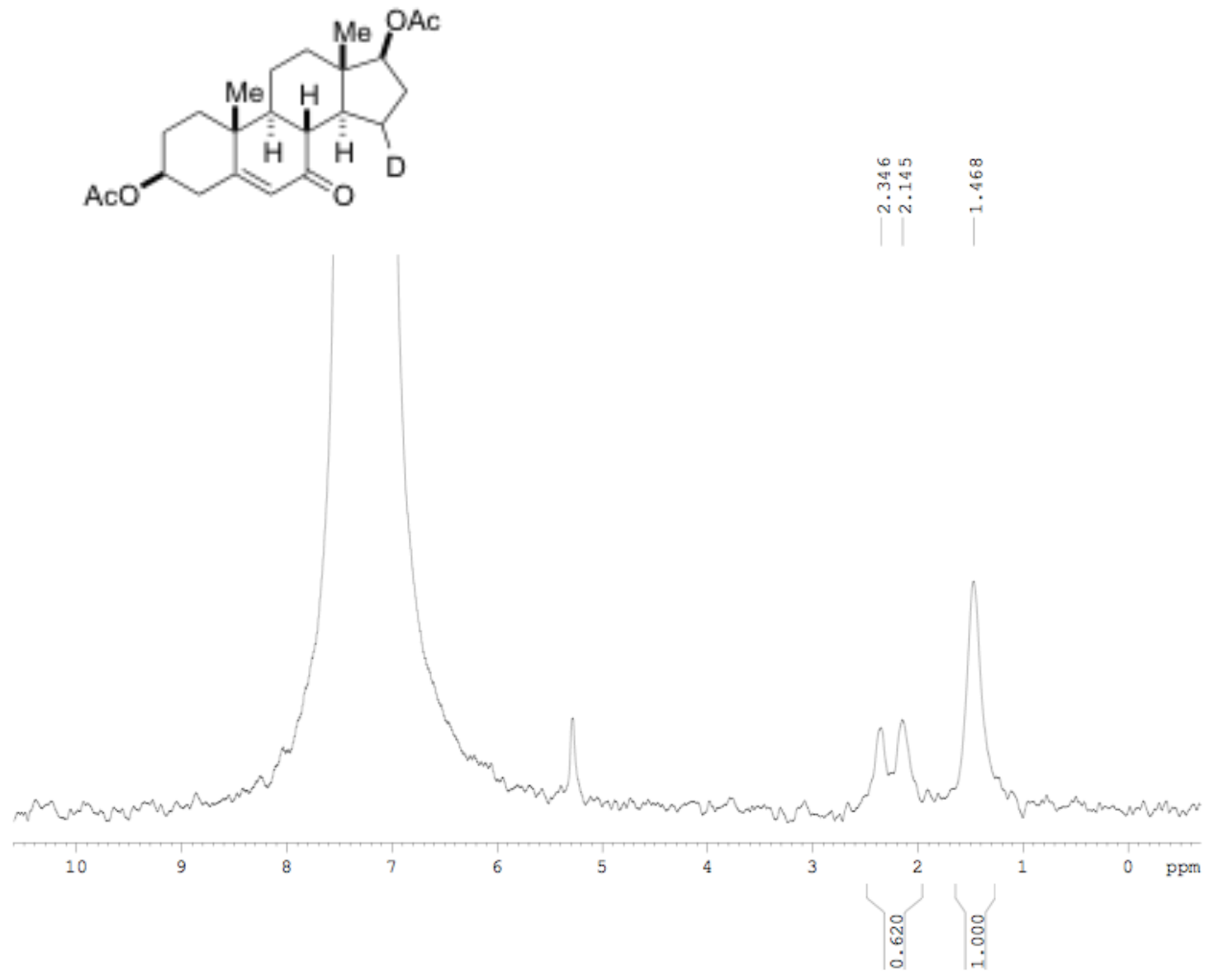

Figure S22. ${ }^{2} \mathrm{H}$ NMR spectrum of compound 17 in $\mathrm{CDCl}_{3} / \mathrm{CH}_{2} \mathrm{Cl}_{2}$. 


\section{Crystallographic structure/parameters for compound 7:}

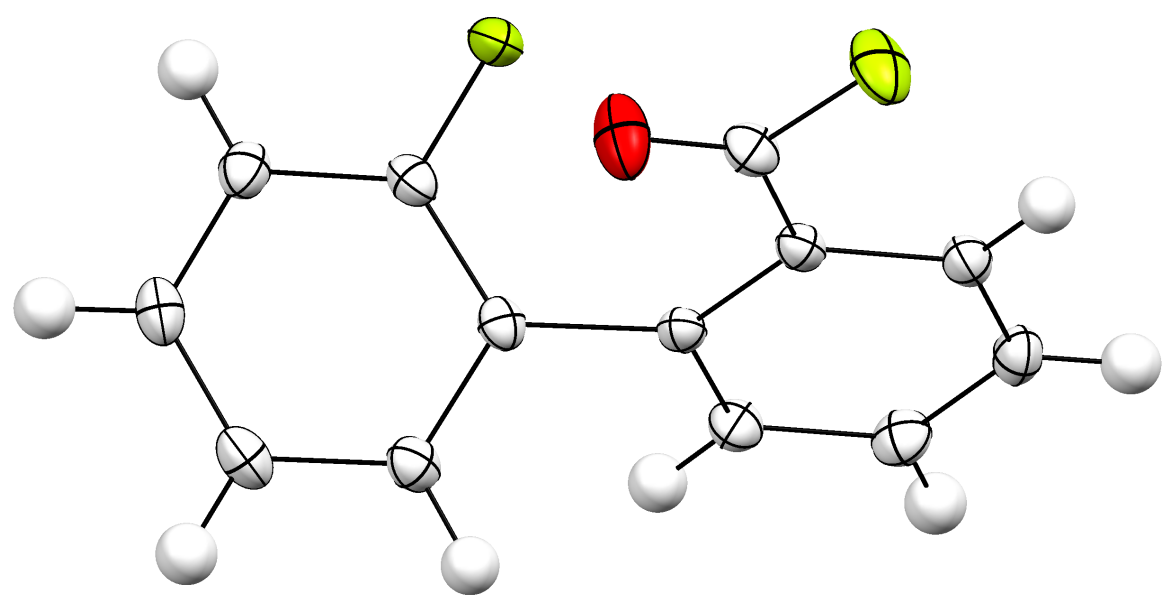

Table S2. Experimental details

\begin{tabular}{|c|c|}
\hline & Compound 7 \\
\hline \multicolumn{2}{|l|}{ Crystal data } \\
\hline Chemical formula & $\mathrm{C}_{13} \mathrm{H}_{8} \mathrm{~F}_{2} \mathrm{O}$ \\
\hline$M_{\mathrm{r}}$ & 218.19 \\
\hline $\begin{array}{l}\text { Crystal system, space } \\
\text { group }\end{array}$ & Monoclinic, $P 2_{1} / n$ \\
\hline Temperature $(\mathrm{K})$ & 110 \\
\hline$a, b, c(\AA)$ & $10.7068(4), 7.8851(3), 11.9482(4)$ \\
\hline$\beta\left(^{\circ}\right)$ & $101.748(3)$ \\
\hline$V\left(\AA^{3}\right)$ & $987.59(6)$ \\
\hline$Z$ & 4 \\
\hline Radiation type & Mo $K \alpha$ \\
\hline$\mu\left(\mathrm{mm}^{-1}\right)$ & 0.12 \\
\hline Crystal size $(\mathrm{mm})$ & $0.45 \times 0.21 \times 0.14$ \\
\hline \multicolumn{2}{|l|}{ Data collection } \\
\hline Diffractometer & SuperNova, Dual, $\mathrm{Cu}$ at zero, Atlas \\
\hline Absorption correction & $\begin{array}{l}\text { Gaussian } \\
\text { CrysAlis PRO 1.171.39.29c (Rigaku Oxford Diffraction, 2017) } \\
\text { Numerical absorption correction based on gaussian integration over a } \\
\text { multifaceted crystal model Empirical absorption correction using } \\
\text { spherical harmonics, implemented in SCALE3 ABSPACK scaling } \\
\text { algorithm. }\end{array}$ \\
\hline
\end{tabular}




\begin{tabular}{|l|l|}
\hline$T_{\min }, T_{\max }$ & $0.551,1.000$ \\
\hline $\begin{array}{l}\text { No. of measured, } \\
\text { independent and } \\
\text { observed }[I>2 \sigma(I)] \\
\text { reflections }\end{array}$ & $14574,2272,2042$ \\
\hline$R_{\text {int }}$ & 0.022 \\
\hline$(\sin \theta / \lambda)_{\max }\left(\AA^{-1}\right)$ & 0.650 \\
\hline & $0.037,0.095,1.11$ \\
\hline Refinement & 2272 \\
\hline $\begin{array}{l}R\left[F^{2}>2 \sigma\left(F^{2}\right)\right], \\
w R\left(F^{2}\right), S\end{array}$ & 145 \\
\hline No. of reflections & H-atom parameters constrained \\
\hline No. of parameters & $0.26,-0.23$ \\
\hline H-atom treatment & \\
\hline$\Delta \rho_{\max }, \Delta \rho_{\text {min }}\left(\mathrm{e} \AA^{-3}\right)$ & \\
\hline
\end{tabular}

Computer programs: CrysAlis PRO 1.171.39.29c (Rigaku OD, 2017), SHELXS2018/3 (Sheldrick, 2018), SHELXL2018/3 (Sheldrick, 2018), SHELXTL v6.10 (Sheldrick, 2008).

Table S3. Single point energies of all structures calculated at the $\operatorname{IEFPCM}_{(\mathrm{CH} 3 \mathrm{CN})} \mathrm{U \omega B} 97 \mathrm{X}-\mathrm{D} / 6$ $311++\mathrm{G}(2 \mathrm{~d}, 2 \mathrm{p})$ level of theory and thermal correction to Gibbs free energy and Gibbs free energy calculated at the UB3LYP/6-31G(d) level of theory. All energies are reported in Hartrees.

\begin{tabular}{|c|c|c|c|c|}
\hline Structure & $\begin{array}{c}\text { Single Point } \\
\text { Energies (E) } \\
\text { IEFPCM }_{(\mathrm{CH} 3 \mathrm{CN})} \mathrm{U} \\
\omega \mathrm{BO7X}^{2} \mathrm{D} / 6- \\
\text { 311++G(2d,2p) }\end{array}$ & $\begin{array}{c}\text { Thermal } \\
\text { Corrections } \\
\text { to Gibbs } \\
\text { Free } \\
\text { Energies } \\
\text { (G) } \\
\text { UB3LYP/6- } \\
\text { 31G(d) }\end{array}$ & $\begin{array}{c}\text { Gibbs Free } \\
\text { Energies } \\
\text { (G) } \\
\text { UB3LYP/6- } \\
\text { 31G(d) }\end{array}$ & $\begin{array}{c}\text { Gibbs Free Energies } \\
(G) \\
\text { IEFPCM }_{(\mathrm{CH} 3 \mathrm{CN})} \mathrm{U} \omega \mathrm{B} 9 \\
7 \mathrm{X}-\mathrm{D} / 6- \\
\text { 311++G(2d,2p)//UB3 } \\
\text { LYP/6-31G(d) }\end{array}$ \\
\hline $\begin{array}{l}\text { Triplet } \\
\text { oxygen }\end{array}$ & -150.32285699 & -0.016200 & -150.336241 & -150.339057 \\
\hline Selectfluor & -944.28446186 & 0.187418 & -943.787594 & -944.0970439 \\
\hline SRD & -844.45193766 & 0.182097 & -843.972822 & -844.2698407 \\
\hline $\begin{array}{c}\text { Protonated } \\
\text { SRD } \\
\end{array}$ & -845.13501006 & 0.198813 & -844.629953 & -844.9361971 \\
\hline
\end{tabular}




\begin{tabular}{|c|c|c|c|c|}
\hline $\begin{array}{c}\text { Selectfluor } \\
\text { Radical } \\
\text { Monocation }\end{array}$ & -844.70387328 & 0.183385 & -844.425749 & -844.5204883 \\
\hline $\mathbf{1 8}$ & -1516.17065190 & 0.401551 & -1515.712323 & -1515.769101 \\
\hline $\mathbf{1}$ & -1271.92149148 & 0.462406 & -1271.417068 & -1271.459085 \\
\hline $\mathbf{1}$ Radical & -1271.25224925 & 0.446993 & -1270.767661 & -1270.805256 \\
\hline $\mathbf{1 3}$ & -1271.67319953 & 0.461157 & -1271.121203 & -1271.212043 \\
\hline $\mathbf{1 4}$ & -1271.66463352 & 0.459187 & -1271.131146 & -1271.205447 \\
\hline Trans Benzil & -689.82546204 & 0.155775 & -689.721941 & -689.669687 \\
\hline Cis Benzil & -689.81703168 & 0.155976 & -689.704037 & -689.6610557 \\
\hline
\end{tabular}

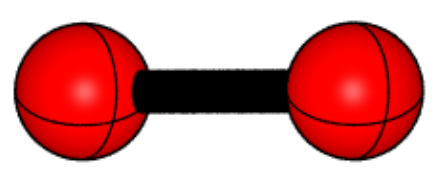

Figure S23. Optimized structure of triplet oxygen.

Zero-point correction $=$

Thermal correction to Energy=

Thermal correction to Enthalpy=

Thermal correction to Gibbs Free Energy=

Sum of electronic and zero-point Energies=

Sum of electronic and thermal Energies=

Sum of electronic and thermal Enthalpies=

Sum of electronic and thermal Free Energies=
0.003779 (Hartree/Particle)

0.006142

0.007086

$-0.016200$

$-150.316262$

$-150.313899$

$-150.312954$

$-150.336241$

Number of Imaginary Frequencies $=0$

E (Single Point Energy) [IEFPCM $\left.{ }_{(\mathrm{CH} 3 \mathrm{CN})} \mathrm{U} \omega \mathrm{B} 97 \mathrm{X}-\mathrm{D} / 6-311++\mathrm{G}(2 \mathrm{~d}, 2 \mathrm{p})\right]=-150.32285699$

03

$\begin{array}{llll}\mathrm{O} & 0.00000000 & 0.00000000 & 0.60729700\end{array}$

O $\quad 0.00000000 \quad 0.00000000-0.60729700$ 


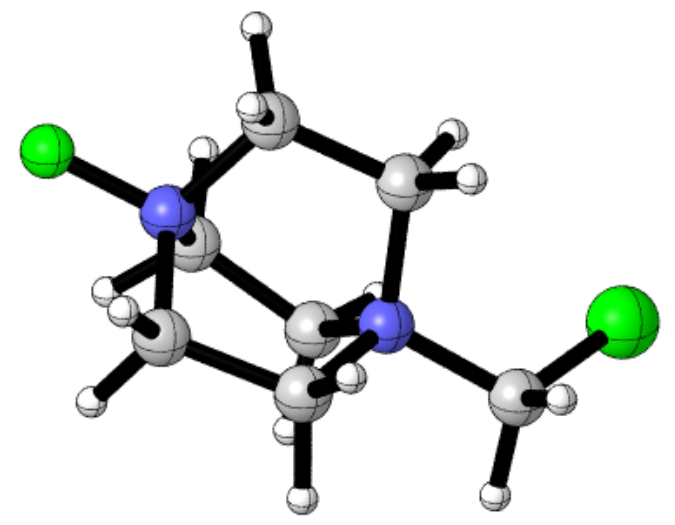

Figure S24. Optimized structure of Selectfluor.

Zero-point correction $=$

Thermal correction to Energy=

Thermal correction to Enthalpy=

Thermal correction to Gibbs Free Energy=

Sum of electronic and zero-point Energies=

Sum of electronic and thermal Energies=

Sum of electronic and thermal Enthalpies=

Sum of electronic and thermal Free Energies=
0.222416 (Hartree/Particle)

0.232118

0.233062

0.187418

$-943.752596$

$-943.742895$

$-943.741951$

$-943.787594$

Number of Imaginary Frequencies $=0$

E (Single Point Energy) [IEFPCM $\left(\mathrm{CH}_{3 \mathrm{CN})} \mathrm{U} \omega \mathrm{B} 97 \mathrm{X}-\mathrm{D} / 6-311++\mathrm{G}(2 \mathrm{~d}, 2 \mathrm{p})\right]=-944.28446186$

21

$\begin{array}{lrrr}\mathrm{C} & 1.27169000 & -0.68320300 & 1.29897200 \\ \mathrm{H} & 1.54765400 & -1.73278800 & 1.42042100 \\ \mathrm{H} & 1.73225500 & -0.09615000 & 2.09601300 \\ \mathrm{C} & -0.26268800 & -0.48757900 & 1.19337700 \\ \mathrm{H} & -0.78868000 & -1.43019300 & 1.04062900 \\ \mathrm{H} & -0.65767600 & -0.01145900 & 2.09332400 \\ \mathrm{C} & 1.77306300 & 1.28658900 & -0.12620800 \\ \mathrm{H} & 2.14397300 & 1.55102500 & -1.11835900 \\ \mathrm{H} & 2.43033200 & 1.71939000 & 0.63090800 \\ \mathrm{C} & 0.28657100 & 1.66609300 & 0.09867700 \\ \mathrm{H} & -0.04853900 & 2.37759100 & -0.65915900 \\ \mathrm{H} & 0.12160300 & 2.10119000 & 1.08667600 \\ \mathrm{C} & -0.22123900 & -0.32443600 & -1.28715200 \\ \mathrm{H} & -0.95017200 & -1.12259400 & -1.43625700 \\ \mathrm{H} & -0.31234600 & 0.39338500 & -2.10541000 \\ \mathrm{C} & 1.21388000 & -0.89581300 & -1.17296900 \\ \mathrm{H} & 1.81793300 & -0.67861300 & -2.05648400 \\ \mathrm{H} & 1.24118600 & -1.96887200 & -0.97349800 \\ \mathrm{~F} & 3.21433600 & -0.55318100 & -0.00245800\end{array}$




$\begin{array}{lrrr}\mathrm{C} & -2.06150400 & 0.84640400 & -0.00160300 \\ \mathrm{H} & -2.21009700 & 1.44565000 & -0.90143200 \\ \mathrm{H} & -2.21167800 & 1.45006200 & 0.89502200 \\ \mathrm{Cl} & -3.16546900 & -0.52200700 & -0.00043900 \\ \mathrm{~N} & -0.57793500 & 0.41211000 & 0.00072600 \\ \mathrm{~N} & 1.86787300 & -0.21114000 & -0.00092200\end{array}$

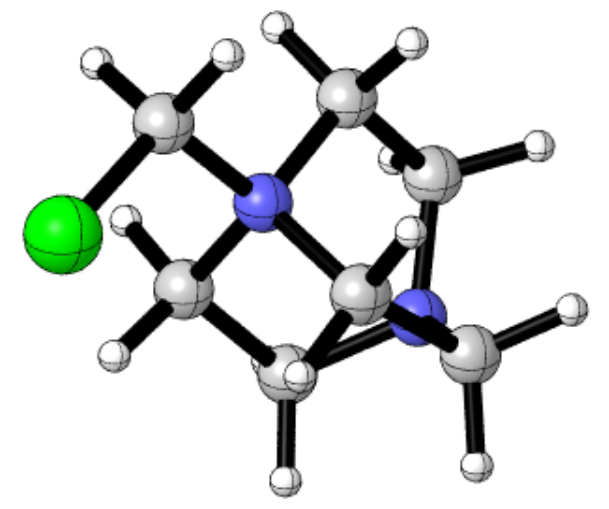

Figure S25. Optimized structure of Selectfluor radical dication (SRD).

Zero-point correction=

Thermal correction to Energy=

Thermal correction to Enthalpy=

Thermal correction to Gibbs Free Energy=

Sum of electronic and zero-point Energies=

Sum of electronic and thermal Energies=

Sum of electronic and thermal Enthalpies=

Sum of electronic and thermal Free Energies=
0.216957 (Hartree/Particle)

0.225898

0.226842

0.182097

$-843.937963$

$-843.929022$

$-843.928078$

$-843.972822$

Number of Imaginary Frequencies $=0$

E (Single Point Energy) [IEFPCM $\left(\mathrm{IH}_{3 \mathrm{CN})} \mathrm{U \omega B}\right.$ B7X-D/6-311++G(2d,2p)] = -844.45193766

\section{2}

$\mathrm{C}$

C

C

$\mathrm{C}$

$\mathrm{H}$

$\mathrm{H}$

$\mathrm{C}$

$\mathrm{H}$

$\mathrm{H}$

$\mathrm{H}$

$\begin{array}{rrr}1.56201700 & -0.90557500 & 1.26647000 \\ 2.22725500 & 1.02158800 & -0.09308500 \\ 1.50494500 & -1.05532300 & -1.17717300 \\ 0.02458500 & -0.53204200 & 1.20868800 \\ 2.07189500 & -0.40572700 & 2.09218400 \\ 1.69550600 & -1.98754700 & 1.34606700 \\ 0.74990000 & 1.55957400 & 0.07824400 \\ 2.64956600 & 1.29153900 & -1.06272900 \\ 2.87170200 & 1.39080500 & 0.70904400 \\ 2.09968700 & -0.82667700 & -2.06565600\end{array}$




$\begin{array}{lrrr}\mathrm{H} & 1.45549300 & -2.13575800 & -1.03050800 \\ \mathrm{C} & 0.06677300 & -0.40598300 & -1.28248000 \\ \mathrm{H} & -0.27571800 & 0.00702400 & 2.10969800 \\ \mathrm{H} & -0.60003800 & -1.41751500 & 1.09121100 \\ \mathrm{H} & 0.50377500 & 2.26383500 & -0.71935500 \\ \mathrm{H} & 0.60993100 & 2.04249700 & 1.04727900 \\ \mathrm{H} & 0.00359000 & 0.29592500 & -2.11644200 \\ \mathrm{H} & -0.69851000 & -1.17575200 & -1.39340600 \\ \mathrm{~N} & 2.08284600 & -0.42334700 & -0.00201800 \\ \mathrm{~N} & -0.21317100 & 0.37498000 & 0.00097000 \\ \mathrm{C} & -1.65807600 & 0.93299300 & 0.00021500 \\ \mathrm{H} & -1.75738000 & 1.54415200 & -0.89820100 \\ \mathrm{H} & -1.75797200 & 1.54510900 & 0.89794000 \\ \mathrm{Cl} & -2.87197900 & -0.34027900 & -0.00029800\end{array}$

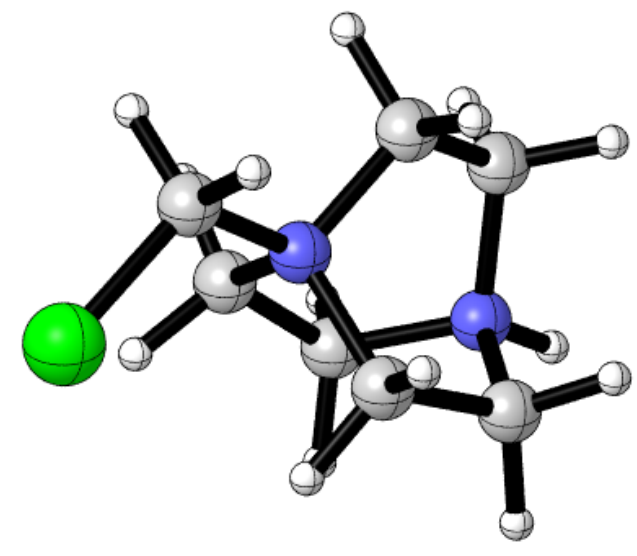

Figure S26. Optimized structure of protonated SRD.

Zero-point correction=

Thermal correction to Energy=

Thermal correction to Enthalpy=

Thermal correction to Gibbs Free Energy=

Sum of electronic and zero-point Energies=

Sum of electronic and thermal Energies=

Sum of electronic and thermal Enthalpies=

Sum of electronic and thermal Free Energies=
0.232714 (Hartree/Particle)

0.241534

0.242478

0.198813

$-844.596052$

$-844.587232$

$-844.586287$

$-844.629953$

Number of Imaginary Frequencies $=0$

E (Single Point Energy) [IEFPCM $\left(\mathrm{ICH}_{3 \mathrm{CN})} \mathrm{U \omega B} \omega 7 \mathrm{X}-\mathrm{D} / 6-311++\mathrm{G}(2 \mathrm{~d}, 2 \mathrm{p})\right]=-845.13501006$

21

$\begin{array}{llll}\text { C } & 1.50798200 & -0.83468700 & 1.30283100\end{array}$ 


$\begin{array}{lrrr}\mathrm{C} & 2.14694700 & 1.08115300 & -0.13960700 \\ \mathrm{C} & 1.42567000 & -1.06188000 & -1.16724700 \\ \mathrm{C} & -0.00693700 & -0.55109600 & 1.18455500 \\ \mathrm{H} & 1.98406900 & -0.26233100 & 2.10198400 \\ \mathrm{H} & 1.70225500 & -1.89630600 & 1.47022600 \\ \mathrm{C} & 0.69995000 & 1.56356900 & 0.11236000 \\ \mathrm{H} & 2.50370500 & 1.32549600 & -1.14245500 \\ \mathrm{H} & 2.84039900 & 1.50331400 & 0.59097300 \\ \mathrm{H} & 2.02356100 & -0.92747400 & -2.07132000 \\ \mathrm{H} & 1.34174500 & -2.13074100 & -0.95909800 \\ \mathrm{C} & 0.05166500 & -0.36856400 & -1.29148400 \\ \mathrm{H} & -0.38813100 & -0.06408300 & 2.08473800 \\ \mathrm{H} & -0.58807100 & -1.45730100 & 1.01242700 \\ \mathrm{H} & 0.40565500 & 2.31374100 & -0.62501700 \\ \mathrm{H} & 0.57477800 & 1.98844100 & 1.11071800 \\ \mathrm{H} & 0.03035200 & 0.36664000 & -2.09922900 \\ \mathrm{H} & -0.74470400 & -1.09528100 & -1.45983100 \\ \mathrm{~N} & 2.16440200 & -0.42851300 & -0.00283800 \\ \mathrm{~N} & -0.25398700 & 0.37918500 & 0.00114000 \\ \mathrm{C} & -1.69771900 & 0.92136000 & 0.00014400 \\ \mathrm{H} & -1.80345200 & 1.53136700 & -0.89840200 \\ \mathrm{H} & -1.80412800 & 1.53306700 & 0.89746100 \\ \mathrm{Cl} & -2.90329100 & -0.36042200 & -0.00033800 \\ \mathrm{H} & 3.13965500 & -0.75519200 & -0.00485800\end{array}$

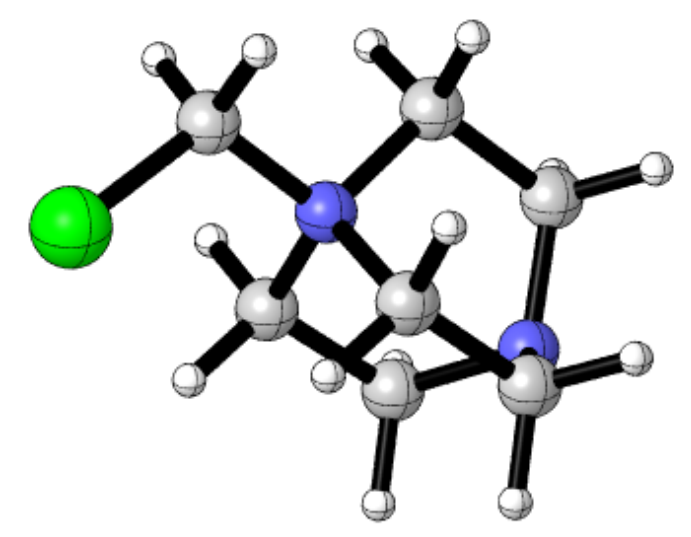

Figure S27. Optimized structure of Selectfluor radical monocation.

Zero-point correction=

Thermal correction to Energy=

Thermal correction to Enthalpy=

Thermal correction to Gibbs Free Energy=
0.218239 (Hartree/Particle)

0.227104

0.228048

0.183385 
Sum of electronic and zero-point Energies=

Sum of electronic and thermal Energies=

Sum of electronic and thermal Enthalpies=

Sum of electronic and thermal Free Energies=
$-844.390894$

$-844.382029$

$-844.381085$

$-844.425749$

Number of Imaginary Frequencies $=0$

E (Single Point Energy) [IEFPCM $\left(\mathrm{CH}_{3 \mathrm{CN})} \mathrm{U} \omega \mathrm{B} 97 \mathrm{X}-\mathrm{D} / 6-311++\mathrm{G}(2 \mathrm{~d}, 2 \mathrm{p})\right]=-844.70387328$

$\begin{array}{lrrr}11 & & & \\ \mathrm{C} & -1.53618600 & -0.94746600 & -1.19704100 \\ \mathrm{C} & -2.19259500 & 1.01684100 & 0.00016200 \\ \mathrm{C} & -1.53609500 & -0.94773400 & 1.19688100 \\ \mathrm{C} & -0.04978300 & -0.47496400 & -1.24562500 \\ \mathrm{H} & -2.07263600 & -0.59334800 & -2.08111600 \\ \mathrm{H} & -1.58331900 & -2.03933000 & -1.19541900 \\ \mathrm{C} & -0.72726300 & 1.55796300 & -0.00017900 \\ \mathrm{H} & -2.72496400 & 1.37905700 & 0.88329700 \\ \mathrm{H} & -2.72542900 & 1.37928300 & -0.88260000 \\ \mathrm{H} & -2.07272200 & -0.59420900 & 2.08108600 \\ \mathrm{H} & -1.58284000 & -2.03961300 & 1.19476100 \\ \mathrm{C} & -0.04988000 & -0.47468100 & 1.24579500 \\ \mathrm{H} & 0.16804600 & 0.16488900 & -2.10460800 \\ \mathrm{H} & 0.66219400 & -1.30062600 & -1.23702400 \\ \mathrm{H} & -0.49298700 & 2.14975100 & 0.88806300 \\ \mathrm{H} & -0.49322200 & 2.14924600 & -0.88881800 \\ \mathrm{H} & 0.16741600 & 0.16554700 & 2.10463400 \\ \mathrm{H} & 0.66240500 & -1.30008400 & 1.23771200 \\ \mathrm{~N} & -2.20929100 & -0.44375700 & 0.00000200 \\ \mathrm{~N} & 0.22860100 & 0.36364900 & 0.00000200 \\ \mathrm{C} & 1.63789400 & 0.91194700 & 0.00000800 \\ \mathrm{H} & 1.75329500 & 1.52173300 & 0.89620900 \\ \mathrm{H} & 1.75329600 & 1.52175200 & -0.89618000 \\ \mathrm{Cl} & 2.89233800 & -0.34439600 & -0.00000200\end{array}$




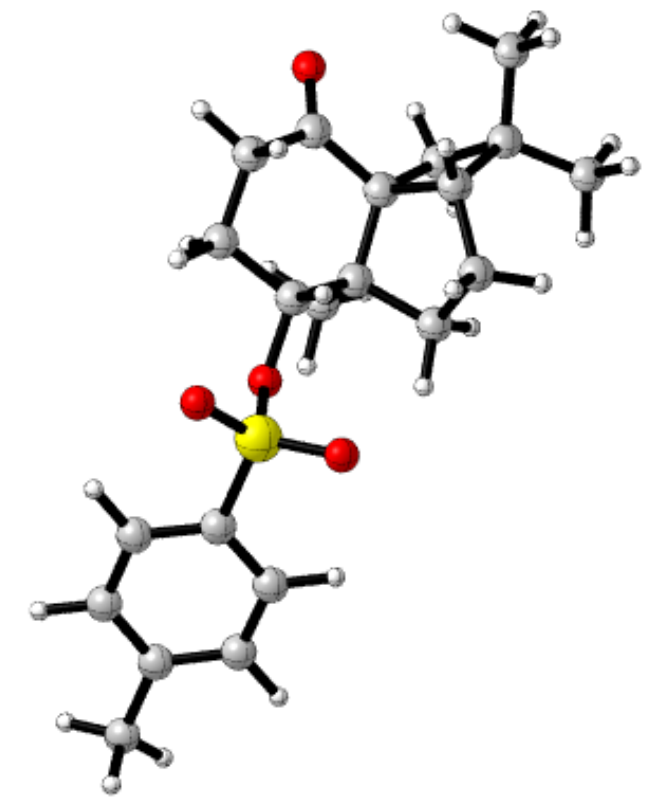

Figure S28. Optimized structure of $\mathbf{1 6 .}$

Zero-point correction=

Thermal correction to Energy=

Thermal correction to Enthalpy=

Thermal correction to Gibbs Free Energy=

Sum of electronic and zero-point Energies=

Sum of electronic and thermal Energies=

Sum of electronic and thermal Enthalpies=

Sum of electronic and thermal Free Energies=
0.458646 (Hartree/Particle)

0.483869

0.484813

0.401551

$-1515.655227$

$-1515.630005$

$-1515.629061$

$-1515.712323$

Number of Imaginary Frequencies $=0$

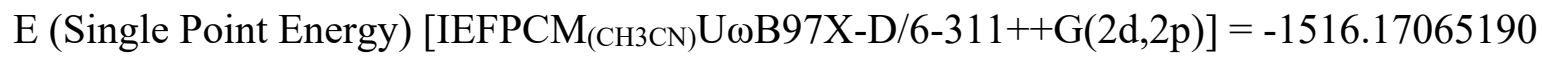

01

$\mathrm{O}$

$\mathrm{C}$

$\mathrm{C}$

$\mathrm{C}$

$\mathrm{C}$

$\mathrm{C}$

$\mathrm{C}$

$\mathrm{H}$

C

C

$\mathrm{H}$

$\mathrm{H}$

$\begin{array}{rrr}-4.04696900 & 2.16281400 & 1.23761300 \\ -3.10212000 & 1.70064200 & 0.61985400 \\ -2.13280100 & 2.61635000 & -0.11363600 \\ -2.84602900 & 0.19971400 & 0.52111100 \\ -0.67276100 & 2.24079600 & 0.17825400 \\ -1.33816000 & -0.22256100 & 0.63775300 \\ -0.46010400 & 0.77293700 & -0.17093000 \\ -2.32144700 & 2.52109100 & -1.19391100 \\ -3.40601700 & -0.49207800 & -0.80112500 \\ -2.28605100 & -1.44132300 & -1.27519800 \\ -2.35740000 & 3.64850100 & 0.16784500 \\ 0.00997300 & 2.85433700 & -0.41671500\end{array}$




$\begin{array}{lrrr}\mathrm{C} & -4.58691500 & -1.10169600 & 0.03634200 \\ \mathrm{C} & -3.87116600 & -0.63701100 & 1.34135600 \\ \mathrm{H} & -4.47530300 & -0.03871400 & 2.02737600 \\ \mathrm{H} & -3.41810000 & -1.46422000 & 1.89456900 \\ \mathrm{C} & -5.89862200 & -0.34640200 & -0.21538300 \\ \mathrm{H} & -5.77212500 & 0.73400000 & -0.09848600 \\ \mathrm{H} & -6.27259600 & -0.54263400 & -1.22886300 \\ \mathrm{H} & -6.67100200 & -0.66653300 & 0.49526000 \\ \mathrm{C} & -4.82696900 & -2.60788000 & -0.07851300 \\ \mathrm{H} & -5.18288300 & -2.88327400 & -1.07964600 \\ \mathrm{H} & -3.92537800 & -3.19401000 & 0.13052500 \\ \mathrm{H} & -5.59419500 & -2.92066500 & 0.64107800 \\ \mathrm{H} & -0.43503200 & 2.41658400 & 1.23382400 \\ \mathrm{C} & -1.32027400 & -1.59610900 & -0.08408600 \\ \mathrm{H} & -1.67531000 & -2.37248200 & 0.60443700 \\ \mathrm{C} & -0.85309000 & -0.30732900 & 2.09457000 \\ \mathrm{H} & 0.18789600 & -0.63923300 & 2.13676000 \\ \mathrm{H} & -0.91849400 & 0.65803400 & 2.60933900 \\ \mathrm{H} & -1.45729000 & -1.02101600 & 2.66175300 \\ \mathrm{H} & -0.66564100 & 0.63801900 & -1.23780300 \\ \mathrm{H} & -0.31135700 & -1.87963900 & -0.39836300 \\ \mathrm{H} & -2.67359300 & -2.40349800 & -1.62535600 \\ \mathrm{H} & -1.75920300 & -0.99924300 & -2.12846000 \\ \mathrm{H} & -3.73955700 & 0.17413600 & -1.60538600 \\ \mathrm{O} & 0.94685900 & 0.41639000 & 0.06414200 \\ \mathrm{~S} & 1.91252300 & 0.37875000 & -1.26378200 \\ \mathrm{O} & 1.50812600 & -0.75376200 & -2.10049300 \\ \mathrm{O} & 1.97275700 & 1.72136900 & -1.84521700 \\ \mathrm{C} & 3.44705700 & 0.00406700 & -0.44222200 \\ \mathrm{C} & 3.82026300 & -1.32856100 & -0.26329300 \\ \mathrm{C} & 4.25789000 & 1.05100200 & -0.00166500 \\ \mathrm{C} & 5.02368400 & -1.60908400 & 0.37919000 \\ \mathrm{H} & 3.18158600 & -2.12504400 & -0.62963500 \\ \mathrm{C} & 5.45718200 & 0.74875200 & 0.63824300 \\ \mathrm{H} & 3.95412800 & 2.07916700 & -0.16645600 \\ \mathrm{H} & 5.85904800 & -0.58018000 & 0.83806100 \\ \mathrm{H} & 5.32134900 & -2.64457100 & 0.52364500 \\ \mathrm{H} & 6.09402000 & 1.55833600 & 0.98568400 \\ \mathrm{H} & 7.17706100 & -0.89533700 & 1.50357000 \\ \mathrm{H} & 7.45435400 & -0.12406000 & 2.22930700 \\ \mathrm{H} & & -1.85810300 & 2.02347800\end{array}$




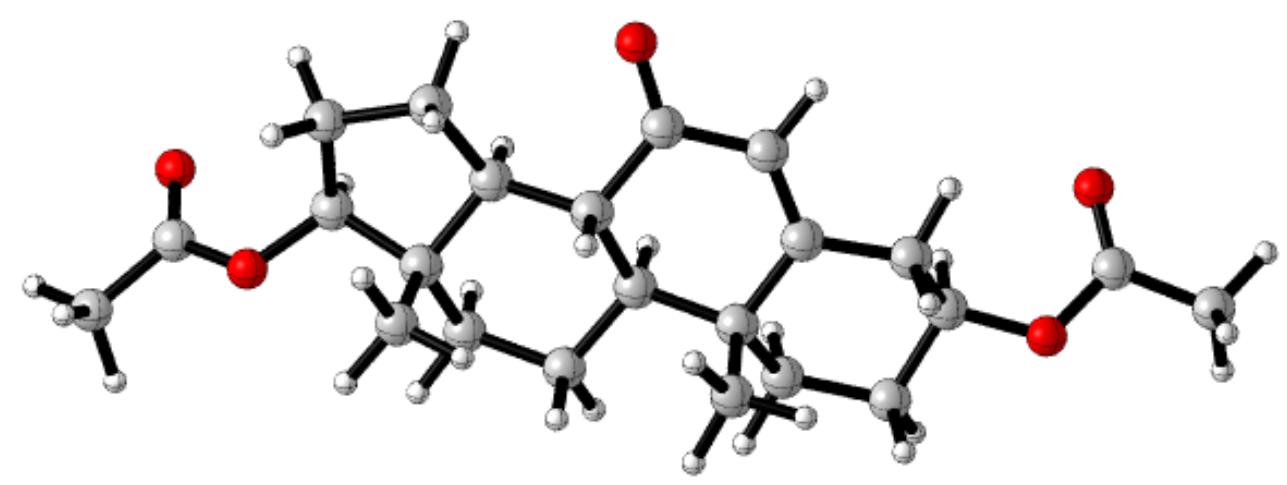

Figure S29. Optimized structure of $\mathbf{1 .}$

Zero-point correction $=$

Thermal correction to Energy=

Thermal correction to Enthalpy=

Thermal correction to Gibbs Free Energy=

Sum of electronic and zero-point Energies $=$

Sum of electronic and thermal Energies=

Sum of electronic and thermal Enthalpies=

Sum of electronic and thermal Free Energies=
0.521688 (Hartree/Particle)

0.549125

0.550069

0.462406

$-1271.357786$

$-1271.330349$

$-1271.329405$

$-1271.417068$

Number of Imaginary Frequencies $=0$

E (Single Point Energy) $\left[\right.$ IEFPCM $\left._{(\mathrm{CH} 3 \mathrm{CN})} \mathrm{U} \omega \mathrm{B} 97 \mathrm{X}-\mathrm{D} / 6-311++\mathrm{G}(2 \mathrm{~d}, 2 \mathrm{p})\right]=-1271.92149148$

01

$\mathrm{C}$

$3.77644000-1.88254300-0.19464800$

$\mathrm{H} \quad 4.18848900 \quad-2.61995100-0.89343500$

$\mathrm{H} \quad 4.00122600 \quad-2.24658200 \quad 0.81578700$

$\begin{array}{llll}\mathrm{C} & 4.45911600 & -0.53853900 & -0.40089600\end{array}$

$\begin{array}{llll}\mathrm{C} & 2.26194900 & -1.75188700 & -0.40966500\end{array}$

$\begin{array}{llll}\mathrm{O} & 5.86774300 & -0.72053000 & -0.11293200\end{array}$

$\begin{array}{llll}\mathrm{C} & 6.73510800 & 0.14289400 & -0.69930500\end{array}$

$\begin{array}{llll}\mathrm{C} & 8.15834100 & -0.19425600 & -0.32033500\end{array}$

$\mathrm{H} \quad 8.39796600 \quad-1.21772600-0.62681200$

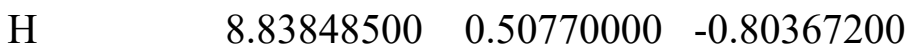

$\begin{array}{llll}\mathrm{H} & 8.28045600 & -0.14265300 & 0.76662200\end{array}$

$\begin{array}{lllll}\mathrm{O} & 6.39808000 & 1.05163100 & -1.42575800\end{array}$

$\begin{array}{llll}\mathrm{C} & 3.86444800 & 0.52321400 & 0.53168900\end{array}$

$\begin{array}{llll}\mathrm{H} & 4.13599700 & 0.24467700 & 1.56090400\end{array}$

$\begin{array}{llll}\mathrm{H} & 4.31915300 & 1.49637600 & 0.32768400\end{array}$

$\mathrm{H} \quad 4.36564000 \quad-0.20699300 \quad-1.43982300$

$\begin{array}{llll}\mathrm{H} & 2.07977800 & -1.49482300 & -1.46262700\end{array}$

$\begin{array}{llll}\mathrm{C} & 2.35768900 & 0.61705500 & 0.41217100\end{array}$

$\begin{array}{llll}\text { C } & 1.55647700 & -0.68522500 & 0.48477300\end{array}$ 


$\begin{array}{lrrr}\mathrm{C} & 1.77168400 & 1.82093700 & 0.27121700 \\ \mathrm{H} & 2.37008900 & 2.72565000 & 0.18757100 \\ \mathrm{H} & 1.79520400 & -2.72837400 & -0.24348800 \\ \mathrm{C} & 1.54628000 & -1.16709700 & 1.96001300 \\ \mathrm{H} & 2.55980400 & -1.23991600 & 2.36500400 \\ \mathrm{H} & 0.99060300 & -0.47753000 & 2.60360300 \\ \mathrm{H} & 1.09108300 & -2.15873200 & 2.04785900 \\ \mathrm{C} & 0.31577900 & 2.03949800 & 0.24352600 \\ \mathrm{C} & 0.10703600 & -0.45016100 & -0.06607300 \\ \mathrm{H} & 0.23329200 & -0.23237800 & -1.13910400 \\ \mathrm{O} & -0.13780800 & 3.15670400 & 0.02925400 \\ \mathrm{C} & -0.56653300 & 0.82299200 & 0.53315300 \\ \mathrm{C} & -0.79528100 & -1.70469500 & 0.02182000 \\ \mathrm{H} & -0.60297000 & 0.73491300 & 1.63059300 \\ \mathrm{C} & -1.99578600 & 0.98423500 & -0.00733300 \\ \mathrm{H} & -1.89555600 & 1.08506000 & -1.10075700 \\ \mathrm{H} & -0.34703700 & -2.51584800 & -0.56295700 \\ \mathrm{H} & -0.83960500 & -2.06532700 & 1.05532900 \\ \mathrm{C} & -2.22969400 & -1.46668500 & -0.48590400 \\ \mathrm{C} & -2.87641900 & -0.27278800 & 0.22837900 \\ \mathrm{H} & -2.20608800 & -1.26717400 & -1.56719300 \\ \mathrm{H} & -2.82532800 & -2.37835700 & -0.34683000 \\ \mathrm{C} & -3.10315400 & -0.58866200 & 1.72401200 \\ \mathrm{H} & -3.58999000 & 0.23705400 & 2.25097700 \\ \mathrm{H} & -3.75097200 & -1.46495700 & 1.82399300 \\ \mathrm{H} & -2.16817700 & -0.80268800 & 2.24902900 \\ \mathrm{C} & -2.88884600 & 2.15642900 & 0.45943800 \\ \mathrm{C} & -4.18800800 & 0.23580000 & -0.39933600 \\ \mathrm{H} & -4.11664800 & 0.20845800 & -1.49078700 \\ \mathrm{C} & -4.33139500 & 1.69796400 & 0.09083500 \\ \mathrm{H} & -4.78563200 & 2.31988200 & -0.68483500 \\ \mathrm{H} & -5.00108000 & 1.72663000 & 0.95700100 \\ \mathrm{H} & -2.78956500 & 2.31466500 & 1.53972300 \\ \mathrm{H} & -2.60266200 & 3.09120400 & -0.02054200 \\ \mathrm{O} & -5.30039300 & -0.60736900 & -0.01969800 \\ \mathrm{C} & -6.39908900 & -0.56313000 & -0.80956800 \\ \mathrm{O} & -6.50126300 & 0.13796900 & -1.79242600 \\ \mathrm{H} & -7.46455400 & -1.50366400 & -0.29330100 \\ \mathrm{H} & -7.07499600 & -2.52554700 & -0.24039300 \\ \mathrm{H} & -7.76048400 & -1.21475700 & 0.72075600 \\ \mathrm{H} & & -1.46932200 & -0.95467400\end{array}$




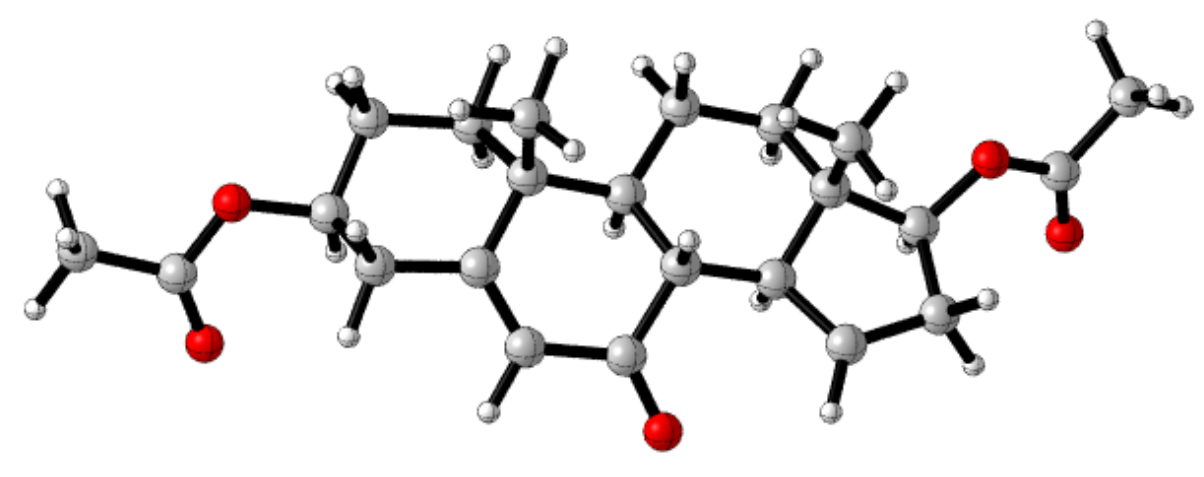

Figure S30. Optimized structure of 1 Radical.

Zero-point correction=

Thermal correction to Energy=

Thermal correction to Enthalpy=

Thermal correction to Gibbs Free Energy=

Sum of electronic and zero-point Energies=

Sum of electronic and thermal Energies=

Sum of electronic and thermal Enthalpies=

Sum of electronic and thermal Free Energies=
0.506749 (Hartree/Particle)

0.534285

0.535229

0.446993

$-1270.707905$

$-1270.680368$

$-1270.679424$

$-1270.767661$

Number of Imaginary Frequencies $=0$

E (Single Point Energy) [IEFPCM $\left(\mathrm{ICH}_{3 \mathrm{CN})} \mathrm{U} \omega \mathrm{B} 97 \mathrm{X}-\mathrm{D} / 6-311++\mathrm{G}(2 \mathrm{~d}, 2 \mathrm{p})\right]=-1271.25224925$

02

$\begin{array}{lrrr}\mathrm{C} & 3.76154500 & -1.87680100 & -0.20346000 \\ \mathrm{H} & 4.17181600 & -2.61044900 & -0.90722600 \\ \mathrm{H} & 3.98769400 & -2.24686300 & 0.80449100 \\ \mathrm{C} & 4.44462900 & -0.53209800 & -0.40295400 \\ \mathrm{C} & 2.24687400 & -1.74368000 & -0.41531200 \\ \mathrm{O} & 5.85387800 & -0.71675500 & -0.12004100 \\ \mathrm{C} & 6.72009100 & 0.15005200 & -0.70322500 \\ \mathrm{C} & 8.14418000 & -0.19018200 & -0.33035500 \\ \mathrm{H} & 8.38238800 & -1.21186000 & -0.64383100 \\ \mathrm{H} & 8.82334300 & 0.51441100 & -0.81122300 \\ \mathrm{H} & 8.26935200 & -0.14537900 & 0.75655700 \\ \mathrm{O} & 6.38142600 & 1.06359700 & -1.42284600 \\ \mathrm{C} & 3.85321800 & 0.52350600 & 0.53844600 \\ \mathrm{H} & 4.12689300 & 0.23721900 & 1.56500800 \\ \mathrm{H} & 4.30845200 & 1.49769300 & 0.34059600 \\ \mathrm{H} & 4.34851400 & -0.19368900 & -1.43943200 \\ \mathrm{H} & 2.06335000 & -1.48073700 & -1.46661400 \\ \mathrm{C} & 2.34620600 & 0.62022800 & 0.42416500\end{array}$




\begin{tabular}{|c|c|c|c|}
\hline $\mathrm{C}$ & 1.54315800 & -0.68174300 & 0.48605400 \\
\hline $\mathrm{C}$ & 1.76177900 & 1.82713800 & 0.30179300 \\
\hline $\mathrm{H}$ & 2.36163400 & 2.73192800 & 0.23033400 \\
\hline $\mathrm{H}$ & 1.77973800 & -2.72074600 & -0.25384300 \\
\hline $\mathrm{C}$ & 1.53083600 & -1.17345800 & 1.95806300 \\
\hline $\mathrm{H}$ & 2.54418900 & -1.25395700 & 2.36213100 \\
\hline $\mathrm{H}$ & 0.97860000 & -0.48539800 & 2.60612300 \\
\hline $\mathrm{H}$ & 1.07096100 & -2.16351200 & 2.03941400 \\
\hline $\mathrm{C}$ & 0.30668200 & 2.04975500 & 0.29052600 \\
\hline $\mathrm{C}$ & 0.09536000 & -0.43855600 & -0.06458300 \\
\hline $\mathrm{H}$ & 0.22526300 & -0.20539900 & -1.13393000 \\
\hline $\mathrm{O}$ & -0.14680200 & 3.17440800 & 0.11909200 \\
\hline $\mathrm{C}$ & -0.57760800 & 0.82795600 & 0.54809200 \\
\hline $\mathrm{C}$ & -0.80735400 & -1.69622100 & 0.00211100 \\
\hline $\mathrm{H}$ & -0.63684900 & 0.72648900 & 1.64228000 \\
\hline $\mathrm{C}$ & -1.99594900 & 0.98902700 & -0.02505600 \\
\hline $\mathrm{H}$ & -1.85054700 & 1.04888800 & -1.12548600 \\
\hline $\mathrm{H}$ & -0.35783600 & -2.49518900 & -0.59809000 \\
\hline $\mathrm{H}$ & -0.84566900 & -2.07581100 & 1.02883800 \\
\hline $\mathrm{C}$ & -2.24798300 & -1.46261500 & -0.49640800 \\
\hline $\mathrm{C}$ & -2.88795400 & -0.26564400 & 0.21714700 \\
\hline $\mathrm{H}$ & -2.23475100 & -1.27069500 & -1.57904600 \\
\hline $\mathrm{H}$ & -2.83909500 & -2.37520900 & -0.34518300 \\
\hline $\mathrm{C}$ & -3.09655800 & -0.56294400 & 1.71848700 \\
\hline $\mathrm{H}$ & -3.47958100 & 0.31149300 & 2.25307200 \\
\hline $\mathrm{H}$ & -3.81872000 & -1.37574200 & 1.84135600 \\
\hline $\mathrm{H}$ & -2.16934200 & -0.86294400 & 2.21355400 \\
\hline $\mathrm{C}$ & -2.90487000 & 2.12024000 & 0.35967000 \\
\hline $\mathrm{C}$ & -4.20697700 & 0.25345300 & -0.39408900 \\
\hline $\mathrm{H}$ & -4.14378800 & 0.24510600 & -1.48668400 \\
\hline $\mathrm{C}$ & -4.33174000 & 1.71256500 & 0.11114400 \\
\hline $\mathrm{H}$ & -4.85063800 & 2.34569300 & -0.62112300 \\
\hline $\mathrm{H}$ & -4.94681100 & 1.73971100 & 1.02547700 \\
\hline $\mathrm{H}$ & -2.57527200 & 3.13919300 & 0.50717300 \\
\hline $\mathrm{O}$ & -5.31686200 & -0.58911300 & -0.01431900 \\
\hline $\mathrm{C}$ & -6.42734500 & -0.52483900 & -0.78738900 \\
\hline $\mathrm{O}$ & -6.53961000 & 0.19570500 & -1.75471700 \\
\hline $\mathrm{C}$ & -7.48907400 & -1.47048000 & -0.27359100 \\
\hline $\mathrm{H}$ & -7.10458800 & -2.49540600 & -0.25074800 \\
\hline $\mathrm{H}$ & -7.76531300 & -1.20302400 & 0.75180900 \\
\hline $\mathrm{H}$ & -8.36652200 & -1.41649200 & -0.91875900 \\
\hline
\end{tabular}




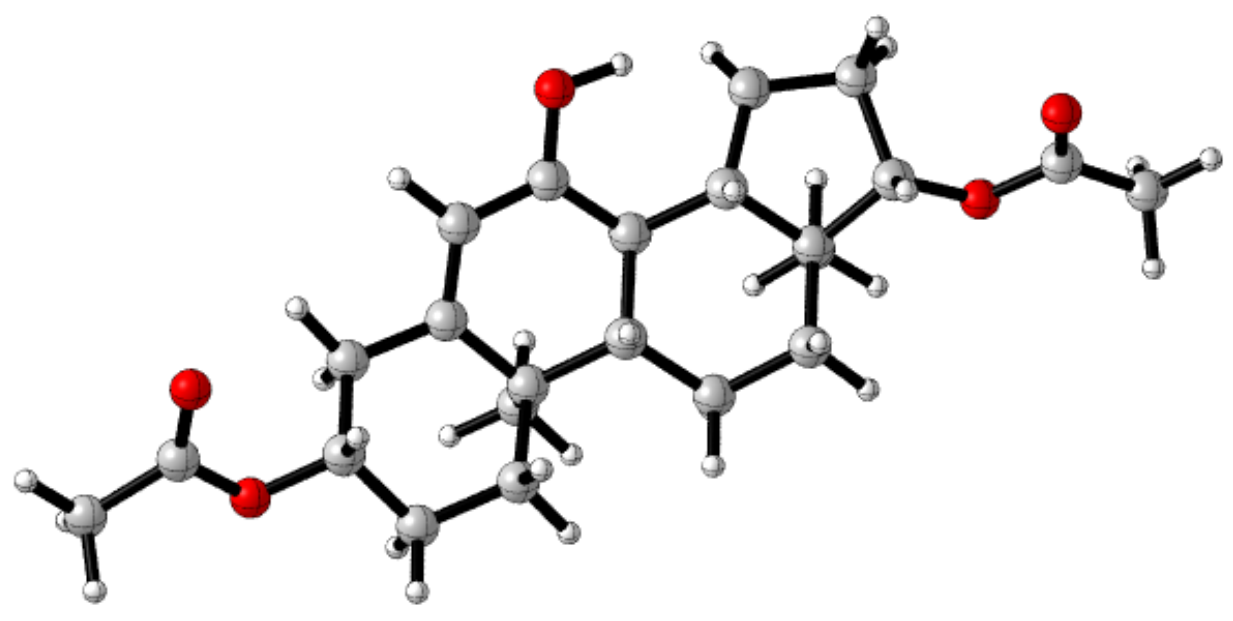

Figure S31. Optimized structure of 13.

Zero-point correction $=$

Thermal correction to Energy=

Thermal correction to Enthalpy=

Thermal correction to Gibbs Free Energy=

Sum of electronic and zero-point Energies $=$

Sum of electronic and thermal Energies=

Sum of electronic and thermal Enthalpies=

Sum of electronic and thermal Free Energies=
0.519823 (Hartree/Particle)

0.547166

0.548110

0.461157

$-1271.062537$

$-1271.035195$

$-1271.034250$

$-1271.121203$

Number of Imaginary Frequencies $=0$

E (Single Point Energy) IEFPCM $\left._{(\mathrm{CH} 3 \mathrm{CN})} \mathrm{U} \omega \mathrm{B} 97 \mathrm{X}-\mathrm{D} / 6-311++\mathrm{G}(2 \mathrm{~d}, 2 \mathrm{p})\right]=-1271.67319953$

12

$\mathrm{C}$

$\mathrm{H}$

$\mathrm{H}$

$\mathrm{C}$

$\mathrm{C}$

$\mathrm{O}$

$\mathrm{C}$

$\mathrm{C}$

$\mathrm{H}$

$\mathrm{H}$

$\mathrm{H}$

$\mathrm{O}$

$\mathrm{C}$

3.81872000

$-1.90331400$

$-0.25671200$

$\begin{array}{lll}4.23132400 & -2.60847700 & -0.98587900\end{array}$

$\begin{array}{lll}4.07904800 & -2.29087700 & 0.73536700\end{array}$

$\begin{array}{lll}4.45992300 & -0.53719400 & -0.44856600\end{array}$

$\begin{array}{lll}2.29871700 & -1.81045200 & -0.44050000\end{array}$

$\begin{array}{lll}5.86437400 & -0.66373100 & -0.19351900\end{array}$

$\begin{array}{llll}6.66813900 & 0.31190500 & -0.72539200\end{array}$

$\begin{array}{llll}8.12023700 & 0.00639800 & -0.47570200\end{array}$

$8.40291300-0.91041400-1.00418200$

$\begin{array}{lll}8.73108400 & 0.83704300 & -0.82943700\end{array}$

$\begin{array}{lll}8.29648100 & -0.16487500 & 0.59077400\end{array}$

$6.22375700 \quad 1.27087600-1.31364800$

$\begin{array}{lll}3.84241500 & 0.49322300 & 0.52503500\end{array}$

$\begin{array}{llll}\mathrm{H} & 4.12438800 & 0.18430700 & 1.54332400\end{array}$

$\mathrm{H}$

4.27142700

1.48186400

0.34951900 


\begin{tabular}{|c|c|c|c|}
\hline $\mathrm{H}$ & 4.32394700 & -0.17758200 & -1.47402000 \\
\hline $\mathrm{H}$ & 2.08404000 & -1.54809600 & -1.48532900 \\
\hline $\mathrm{C}$ & 2.34709000 & 0.53742700 & 0.44511200 \\
\hline $\mathrm{C}$ & 1.57768000 & -0.77575500 & 0.48442400 \\
\hline $\mathrm{C}$ & 1.72902900 & 1.76111600 & 0.34653300 \\
\hline $\mathrm{H}$ & 2.30882000 & 2.67533400 & 0.25990000 \\
\hline $\mathrm{H}$ & 1.85299200 & -2.79490500 & -0.26931700 \\
\hline $\mathrm{C}$ & 1.60387600 & -1.28040400 & 1.95807500 \\
\hline $\mathrm{H}$ & 2.62683200 & -1.40645000 & 2.31955900 \\
\hline $\mathrm{H}$ & 1.09851200 & -0.59240300 & 2.64380600 \\
\hline $\mathrm{H}$ & 1.11219700 & -2.25446500 & 2.02573900 \\
\hline $\mathrm{C}$ & 0.32674400 & 1.87520300 & 0.37800500 \\
\hline $\mathrm{C}$ & 0.11789800 & -0.55874200 & -0.04051200 \\
\hline $\mathrm{H}$ & 0.21977600 & -0.30632200 & -1.10766300 \\
\hline $\mathrm{O}$ & -0.17505400 & 3.06422300 & 0.21506600 \\
\hline $\mathrm{C}$ & -0.55370200 & 0.68470200 & 0.61393400 \\
\hline $\mathrm{C}$ & -0.79760200 & -1.80344100 & 0.03117600 \\
\hline $\mathrm{H}$ & -0.59747900 & 0.55306300 & 1.70748700 \\
\hline $\mathrm{C}$ & -1.96848900 & 0.88496900 & 0.06688300 \\
\hline $\mathrm{H}$ & -1.85952600 & 1.00906900 & -1.02687500 \\
\hline $\mathrm{H}$ & -0.34837200 & -2.60790900 & -0.55945500 \\
\hline $\mathrm{H}$ & -0.85540500 & -2.17296900 & 1.06013400 \\
\hline $\mathrm{C}$ & -2.22550400 & -1.54369600 & -0.49979300 \\
\hline $\mathrm{C}$ & -2.89156500 & -0.36780500 & 0.23104800 \\
\hline $\mathrm{H}$ & -2.18093300 & -1.32601200 & -1.57615800 \\
\hline $\mathrm{H}$ & -2.82330200 & -2.45521000 & -0.38770300 \\
\hline $\mathrm{C}$ & -3.16899300 & -0.71386300 & 1.70841300 \\
\hline $\mathrm{H}$ & -3.63688900 & 0.11531400 & 2.24735400 \\
\hline $\mathrm{H}$ & -3.85490900 & -1.56325900 & 1.76097500 \\
\hline $\mathrm{H}$ & -2.26043000 & -0.98773500 & 2.25333000 \\
\hline $\mathrm{C}$ & -2.84880100 & 2.00323100 & 0.55811200 \\
\hline $\mathrm{C}$ & -4.16691400 & 0.21939200 & -0.42615600 \\
\hline $\mathrm{H}$ & -4.06243500 & 0.21908200 & -1.51599500 \\
\hline $\mathrm{C}$ & -4.24943200 & 1.69347800 & 0.09033000 \\
\hline $\mathrm{H}$ & -4.60734800 & 2.36161700 & -0.70253600 \\
\hline $\mathrm{H}$ & -4.97136600 & 1.77471300 & 0.91126900 \\
\hline $\mathrm{H}$ & -2.68508000 & 2.51231000 & 1.50876800 \\
\hline $\mathrm{O}$ & -5.31202000 & -0.56686200 & -0.08841500 \\
\hline $\mathrm{C}$ & -6.42366800 & -0.35497200 & -0.86218500 \\
\hline $\mathrm{O}$ & -6.45052400 & 0.46849100 & -1.74631900 \\
\hline $\mathrm{C}$ & -7.54779300 & -1.26914100 & -0.45256500 \\
\hline $\mathrm{H}$ & -7.24797500 & -2.31483000 & -0.57819000 \\
\hline $\mathrm{H}$ & -7.78823900 & -1.12283200 & 0.60545400 \\
\hline $\mathrm{H}$ & -8.42483200 & -1.06023600 & -1.06521000 \\
\hline $\mathrm{H}$ & -1.17155800 & 3.04456700 & 0.20589500 \\
\hline
\end{tabular}




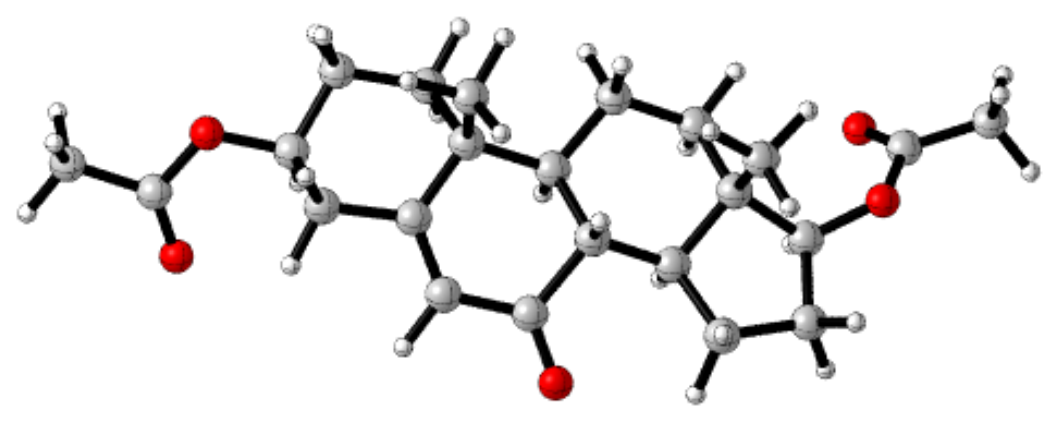

Figure S32. Optimized structure of 14.

Zero-point correction=

Thermal correction to Energy=

Thermal correction to Enthalpy=

Thermal correction to Gibbs Free Energy=

Sum of electronic and zero-point Energies=

Sum of electronic and thermal Energies=

Sum of electronic and thermal Enthalpies=

Sum of electronic and thermal Free Energies=
0.518273 (Hartree/Particle)

0.545896

0.546840

0.459187

$-1271.072060$

$-1271.044437$

$-1271.043493$

$-1271.131146$

Number of Imaginary Frequencies $=0$

E (Single Point Energy) [IEFPCM $(\mathrm{CH} 3 \mathrm{CN}) \mathrm{U} \omega \mathrm{B} 97 \mathrm{X}-\mathrm{D} / 6-311++\mathrm{G}(2 \mathrm{~d}, 2 \mathrm{p})]=-1271.66463352$

12

$\begin{array}{lrrr}\mathrm{C} & 3.62262300 & -1.96013900 & 0.13823700 \\ \mathrm{H} & 3.98629100 & -2.81761100 & -0.43755600 \\ \mathrm{H} & 3.86674200 & -2.16087800 & 1.18829500 \\ \mathrm{C} & 4.33921200 & -0.69679100 & -0.31323900 \\ \mathrm{C} & 2.10891700 & -1.81531100 & -0.06526100 \\ \mathrm{O} & 5.73929200 & -0.86068200 & -0.04060700 \\ \mathrm{C} & 6.59391600 & -0.06263700 & -0.75347700 \\ \mathrm{C} & 8.02810000 & -0.40111500 & -0.44561300 \\ \mathrm{H} & 8.24845100 & -1.42123300 & -0.77795200 \\ \mathrm{H} & 8.68435000 & 0.30204000 & -0.95857000 \\ \mathrm{H} & 8.20501500 & -0.36447500 & 0.63380300 \\ \mathrm{O} & 6.20480500 & 0.78641300 & -1.52269900 \\ \mathrm{C} & 3.80252100 & 0.53054500 & 0.44741400 \\ \mathrm{H} & 4.07950800 & 0.41208200 & 1.50562200 \\ \mathrm{H} & 4.28430800 & 1.43923400 & 0.08096100 \\ \mathrm{H} & 4.21179600 & -0.53448100 & -1.38881600 \\ \mathrm{H} & 1.90409100 & -1.74250500 & -1.14211500 \\ \mathrm{C} & 2.30020800 & 0.65310900 & 0.36398100\end{array}$




$\begin{array}{lrrr}\mathrm{C} & 1.45904000 & -0.58502300 & 0.64618900 \\ \mathrm{C} & 1.77804100 & 1.86917000 & 0.07438400 \\ \mathrm{H} & 2.40953100 & 2.73066800 & -0.11843000 \\ \mathrm{H} & 1.61266400 & -2.72492100 & 0.28604400 \\ \mathrm{C} & 1.44933000 & -0.80517400 & 2.18459800 \\ \mathrm{H} & 2.46440100 & -0.88484600 & 2.58079100 \\ \mathrm{H} & 0.95824300 & 0.01612200 & 2.71696400 \\ \mathrm{H} & 0.93232900 & -1.73602500 & 2.43315700 \\ \mathrm{C} & 0.35346000 & 2.15743000 & 0.07853700 \\ \mathrm{C} & 0.02319300 & -0.36960800 & 0.06310000 \\ \mathrm{H} & 0.13672900 & -0.33651300 & -1.03014900 \\ \mathrm{O} & -0.08905900 & 3.26996300 & -0.12350800 \\ \mathrm{C} & -0.63261400 & 0.96219600 & 0.46690400 \\ \mathrm{C} & -0.95342100 & -1.54851000 & 0.37322500 \\ \mathrm{H} & -0.70635800 & 1.06802300 & 1.55878600 \\ \mathrm{C} & -1.98670000 & 1.15267800 & -0.19159800 \\ \mathrm{H} & -1.84512300 & 1.02581100 & -1.27488000 \\ \mathrm{H} & -0.52142500 & -2.46763500 & -0.03355000 \\ \mathrm{H} & -1.03651800 & -1.69355400 & 1.45441600 \\ \mathrm{C} & -2.34923800 & -1.32952900 & -0.23409100 \\ \mathrm{C} & -2.97111700 & -0.01384600 & 0.23562700 \\ \mathrm{H} & -2.28744200 & -1.33875300 & -1.32889500 \\ \mathrm{H} & -2.99026600 & -2.17639300 & 0.04348600 \\ \mathrm{C} & -3.30318500 & -0.03744600 & 1.73832800 \\ \mathrm{H} & -3.73504400 & 0.90408700 & 2.08770900 \\ \mathrm{H} & -4.04067300 & -0.82093100 & 1.93536200 \\ \mathrm{H} & -2.43063700 & -0.25371200 & 2.36072400 \\ \mathrm{C} & -2.79637200 & 2.42808100 & 0.03256200 \\ \mathrm{C} & -4.21321700 & 0.49509300 & -0.57755500 \\ \mathrm{H} & -4.06340600 & 0.22880500 & -1.62827000 \\ \mathrm{C} & -4.24723500 & 2.02687800 & -0.36052800 \\ \mathrm{H} & -4.59781300 & 2.53481000 & -1.26311700 \\ \mathrm{H} & -4.95369100 & 2.27262600 & 0.43729800 \\ \mathrm{H} & -2.74354100 & 2.76085600 & 1.07375700 \\ \mathrm{H} & -2.41142000 & 3.24872200 & -0.58667900 \\ \mathrm{H} & -5.45447600 & -0.06005800 & -0.14877800 \\ \mathrm{H} & -5.87943200 & -1.21997000 & -0.75062300 \\ \mathrm{H} & -5.19360600 & -1.83206900 & -1.53646600 \\ \mathrm{H} & -7.26628400 & -1.58264400 & -0.29740300 \\ \mathrm{H} & -7.5458300 & -1.61836900 & 0.79533600 \\ \mathrm{H} & -0.81600800 & -0.63017200 \\ \mathrm{H} & -2.54819000 & -0.71909100\end{array}$




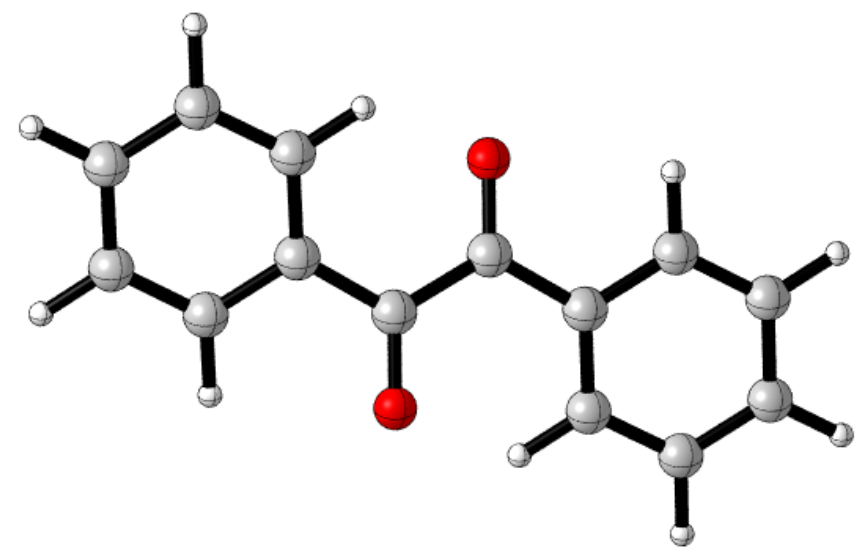

Figure S33. Optimized structure of trans benzil.

Zero-point correction=

Thermal correction to Energy=

Thermal correction to Enthalpy=

Thermal correction to Gibbs Free Energy=

Sum of electronic and zero-point Energies $=$

Sum of electronic and thermal Energies=

Sum of electronic and thermal Enthalpies $=$

Sum of electronic and thermal Free Energies=
0.198572 (Hartree/Particle)

0.211383

0.212327

0.155775

$-689.679144$

$-689.666334$

$-689.665389$

$-689.721941$

Number of Imaginary Frequencies $=0$

E (Single Point Energy) [IEFPCM $\left.{ }_{(\mathrm{CH} 3 \mathrm{CN})} \mathrm{U} \omega \mathrm{B} 97 \mathrm{X}-\mathrm{D} / 6-311++\mathrm{G}(2 \mathrm{~d}, 2 \mathrm{p})\right]=-689.82546204$

03

\begin{tabular}{|c|c|c|c|}
\hline & 0.51953400 & 0.52764800 & 0.11663400 \\
\hline & 1.96885000 & 0.19907000 & 0.06360200 \\
\hline & 2.86477600 & 1.27940500 & -0.07259300 \\
\hline & 2.47947300 & -1.10309800 & 0.17346500 \\
\hline & 4.23437000 & 1.05251300 & -0.13559000 \\
\hline & 2.46647900 & 2.28580200 & -0.14349500 \\
\hline & 3.85587100 & -1.32007400 & 0.11160200 \\
\hline & 1.80853100 & -1.94170800 & 0.31332700 \\
\hline & 4.73689100 & -0.24908700 & -0.04570600 \\
\hline & 4.91250500 & 1.89267800 & -0.25837500 \\
\hline & 4.23851400 & -2.33311000 & 0.19901600 \\
\hline & 5.80806000 & -0.42585700 & -0.09173000 \\
\hline & -0.51951900 & -0.52755700 & -0.11663300 \\
\hline & -1.96884400 & -0.19902300 & -0.06357900 \\
\hline & -2.47952700 & 1.10316000 & -0.17296000 \\
\hline & -2.86473300 & -1.27945100 & 0.07215000 \\
\hline & 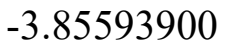 & 1.32004 & -0.111122( \\
\hline
\end{tabular}




$\begin{array}{lrrc}\mathrm{H} & -1.80860800 & 1.94185900 & -0.31241500 \\ \mathrm{C} & -4.23434200 & -1.05265300 & 0.13512400 \\ \mathrm{H} & -2.46638700 & -2.28585300 & 0.14270400 \\ \mathrm{C} & -4.73692100 & 0.24895500 & 0.04569800 \\ \mathrm{H} & -4.23862300 & 2.33310000 & -0.19815700 \\ \mathrm{H} & -4.91244500 & -1.89290000 & 0.25753100 \\ \mathrm{H} & -5.80810200 & 0.42565700 & 0.09170800 \\ \mathrm{O} & -0.16395900 & -1.70510700 & -0.34990200 \\ \mathrm{O} & 0.16401300 & 1.70525800 & 0.34981800\end{array}$

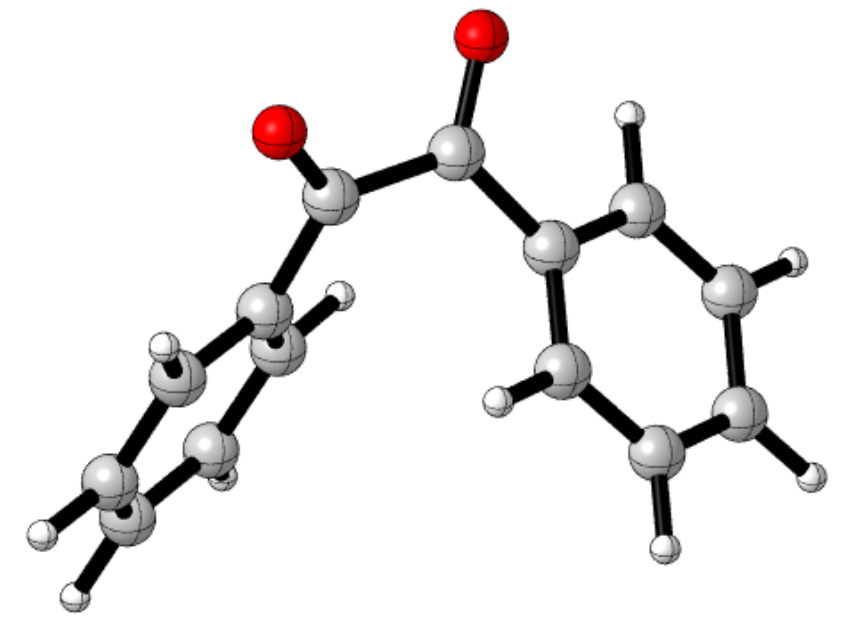

Figure S34. Optimized structure of cis benzil.

Zero-point correction $=$

Thermal correction to Energy=

Thermal correction to Enthalpy=

Thermal correction to Gibbs Free Energy=

Sum of electronic and zero-point Energies=

Sum of electronic and thermal Energies=

Sum of electronic and thermal Enthalpies $=$

Sum of electronic and thermal Free Energies=
0.197541 (Hartree/Particle)

0.210456

0.211400

0.155976

$-689.662472$

$-689.649557$

$-689.648613$

$-689.704037$

Number of Imaginary Frequencies $=0$

E (Single Point Energy) [IEFPCM $\left.{ }_{(\mathrm{CH} 3 \mathrm{CN})} \mathrm{U \omega B} \omega 7 \mathrm{X}-\mathrm{D} / 6-311++\mathrm{G}(2 \mathrm{~d}, 2 \mathrm{p})\right]=-689.81703168$
03
$\begin{array}{lllr}\mathrm{C} & -0.73368300 & 1.60686200 & 0.18885300 \\ \mathrm{C} & -1.55965400 & 0.38678600 & -0.03826600\end{array}$
C $\quad-2.72316800 \quad 0.23116300 \quad 0.73853100$
C $\quad-1.27718300 \quad-0.54654600 \quad-1.04898500$ 


$\begin{array}{lrrr}\mathrm{C} & -3.55913800 & -0.86298200 & 0.53434800 \\ \mathrm{H} & -2.95161700 & 0.96950600 & 1.49976900 \\ \mathrm{C} & -2.12079500 & -1.63708700 & -1.24689400 \\ \mathrm{H} & -0.40796500 & -0.41412200 & -1.68350100 \\ \mathrm{C} & -3.26020000 & -1.80280700 & -0.45526600 \\ \mathrm{H} & -4.44938300 & -0.97907400 & 1.14653600 \\ \mathrm{H} & -1.89215100 & -2.35383900 & -2.03071400 \\ \mathrm{H} & -3.91684000 & -2.65320600 & -0.61654300 \\ \mathrm{C} & 0.73370300 & 1.60714500 & -0.18903600 \\ \mathrm{C} & 1.55968500 & 0.38697900 & 0.03822800 \\ \mathrm{C} & 1.27782900 & -0.54554300 & 1.04981600 \\ \mathrm{C} & 2.72252000 & 0.23068800 & -0.73939300 \\ \mathrm{C} & 2.12149700 & -1.63602000 & 1.24792400 \\ \mathrm{H} & 0.40906700 & -0.41249500 & 1.68483300 \\ \mathrm{C} & 3.55852100 & -0.86341400 & -0.53506500 \\ \mathrm{H} & 2.95045700 & 0.96849700 & -1.50130700 \\ \mathrm{C} & 3.26024100 & -1.80244300 & 0.45550800 \\ \mathrm{H} & 1.89340300 & -2.35216700 & 2.03245500 \\ \mathrm{H} & 4.44827400 & -0.98008400 & -1.14785600 \\ \mathrm{H} & 3.91691600 & -2.65279400 & 0.61690200 \\ \mathrm{O} & 1.26258200 & 2.64650900 & -0.62190900 \\ \mathrm{O} & -1.26273500 & 2.64637700 & 0.62160900\end{array}$

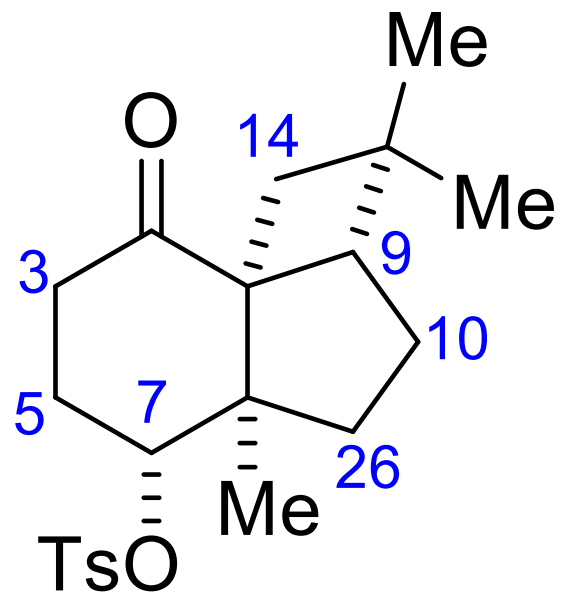

Figure S35. Depiction of 18 with Pertinent Carbon Atoms Labelled. Top Hydrogen is Denoted by $(\mathrm{T})$, and the Bottom Hydrogen is Denoted by (B).

Table S4. Single point energies of all structures calculated at the $\operatorname{IEFPCM}_{(\mathrm{CH} 3 \mathrm{CN})} \mathrm{U} \omega \mathrm{B} 97 \mathrm{X}-\mathrm{D} / 6$ $311++\mathrm{G}(2 \mathrm{~d}, 2 \mathrm{p})$ level of theory and thermal correction to Gibbs free energy and Gibbs free energy calculated at the UB3LYP/6-31G(d) level of theory. All energies are reported in Hartrees. 


\begin{tabular}{|c|c|c|c|c|}
\hline Structure & $\begin{array}{c}\text { Single Point } \\
\text { Energies (E) } \\
\text { IEFPCM }_{(\mathrm{CH} 3 \mathrm{CN})} \mathbf{U} \\
\omega \text { BO7X-D/6- } \\
\text { 311++G(2d,2p) }\end{array}$ & $\begin{array}{c}\text { Thermal } \\
\text { Corrections } \\
\text { to Gibbs } \\
\text { Free } \\
\text { Energies } \\
\text { (G) } \\
\text { UB3LYP/6- } \\
\text { 31G(d) } \\
\end{array}$ & $\begin{array}{c}\text { Gibbs Free } \\
\text { Energies } \\
\text { (G) } \\
\text { UB3LYP/6- } \\
\text { 31G(d) }\end{array}$ & $\begin{array}{c}\text { Gibbs Free Energies } \\
\text { (G) } \\
\text { IEFPCM }_{(\mathrm{CH} 3 \mathrm{CN})} \mathrm{U \omega B} 9 \\
7 \mathrm{X}-\mathrm{D} / 6- \\
311++\mathrm{G}(2 \mathrm{~d}, 2 \mathrm{p}) / / \mathrm{UB3} \\
\mathrm{LYP} / 6-31 \mathrm{G}(\mathrm{d})\end{array}$ \\
\hline C9 SRD & -2360.62791972 & 0.602774 & -2359.737429 & -2360.025146 \\
\hline C10 (B) SRD & -2360.62251326 & 0.601795 & -2359.729544 & -2360.020718 \\
\hline C10 (T) SRD & -2360.63295839 & 0.600602 & -2359.743887 & -2360.032356 \\
\hline C26 (T) SRD & -2360.62067530 & 0.603582 & -2359.738457 & -2360.017093 \\
\hline C26 (B) SRD & -2360.62432526 & 0.601230 & -2359.734478 & -2360.023095 \\
\hline C14 (T) SRD & -2360.63416875 & 0.600905 & -2359.737626 & -2360.033264 \\
\hline C14 (B) SRD & -2360.61793073 & 0.601539 & -2359.716537 & -2360.016392 \\
\hline C3 (T) SRD & -2360.62349078 & 0.599666 & -2359.733717 & -2360.023825 \\
\hline C3 (B) SRD & -2360.62293097 & 0.599905 & -2359.739022 & -2360.023026 \\
\hline C7 SRD & -2360.61371366 & 0.602642 & -2359.731539 & -2360.011072 \\
\hline C5 (B) SRD & -2360.63094606 & 0.601547 & -2359.745141 & -2360.029399 \\
\hline C5 (T) SRD & -2360.62620885 & 0.602658 & -2359.736828 & -2360.023551 \\
\hline
\end{tabular}

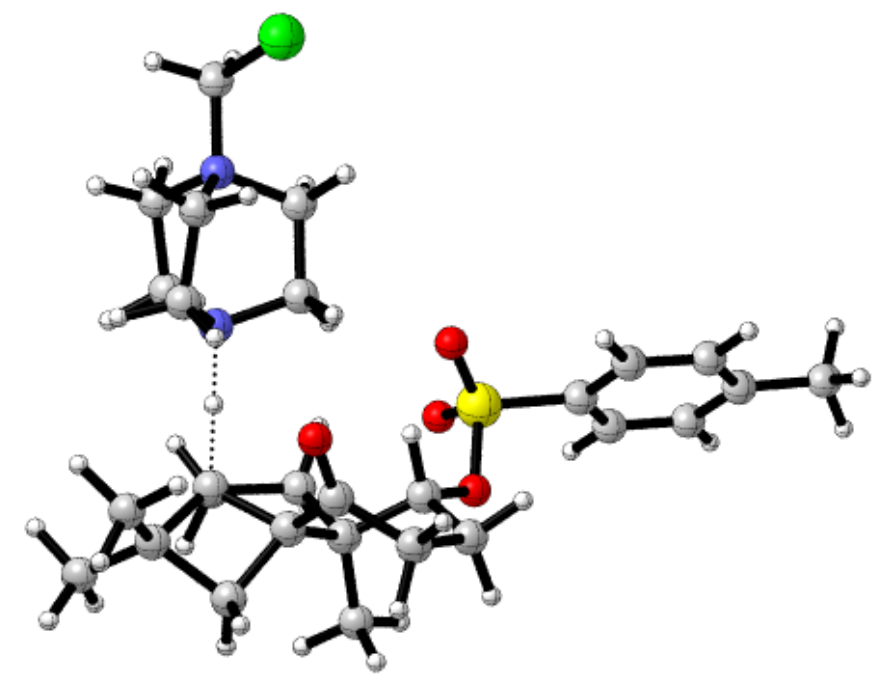

Figure S36. Transition state involving carbon C9 using SRD.

Zero-point correction $=$

Thermal correction to Energy=

Thermal correction to Enthalpy=

Thermal correction to Gibbs Free Energy=

Sum of electronic and zero-point Energies=
0.672031 (Hartree/Particle)

0.707715

0.708659

0.602774

$-2359.668171$ 
Sum of electronic and thermal Energies=

$-2359.632488$

Sum of electronic and thermal Enthalpies=

$-2359.631544$

Sum of electronic and thermal Free Energies=

$-2359.737429$

Number of Imaginary Frequencies $=1$

E (Single Point Energy) $\left[\operatorname{IEFPCM}_{(\mathrm{CH} 3 \mathrm{CN})} \mathrm{U \omega B}\right.$ B97X-D/6-311++G(2d,2p)] = -2360.62791972

$\begin{array}{lrrr}22 & & & \\ \mathrm{C} & -1.55431800 & 1.26669800 & 0.06148000 \\ \mathrm{C} & -3.15169600 & 0.82568300 & -1.73491900 \\ \mathrm{C} & -3.86598900 & 0.71891200 & 0.59609100 \\ \mathrm{C} & -1.83887600 & 2.75603700 & -0.23428900 \\ \mathrm{H} & -0.66160300 & 0.94789500 & -0.48119200 \\ \mathrm{H} & -1.39305200 & 1.08104500 & 1.12278000 \\ \mathrm{C} & -3.74285000 & 2.25743400 & -1.72126900 \\ \mathrm{H} & -3.89578300 & 0.11011700 & -2.09176300 \\ \mathrm{H} & -2.27220900 & 0.76145000 & -2.37844500 \\ \mathrm{H} & -4.75944200 & 0.22332500 & 0.21130900 \\ \mathrm{H} & -3.59927700 & 0.27454900 & 1.55476100 \\ \mathrm{C} & -4.06809100 & 2.24856800 & 0.72510900 \\ \mathrm{H} & -1.35977700 & 3.09937200 & -1.15429100 \\ \mathrm{H} & -1.51990100 & 3.39823700 & 0.58730600 \\ \mathrm{H} & -4.83443100 & 2.25805000 & -1.75910900 \\ \mathrm{H} & -3.36245000 & 2.85504600 & -2.55253600 \\ \mathrm{H} & -5.12268400 & 2.52708500 & 0.66785800 \\ \mathrm{H} & -3.65499200 & 2.64624200 & 1.65193500 \\ \mathrm{~N} & -2.74250800 & 0.45291900 & -0.35130200 \\ \mathrm{~N} & -3.34085900 & 2.94385900 & -0.42160200 \\ \mathrm{C} & -3.71536200 & 4.41818100 & -0.52755300 \\ \mathrm{H} & -4.77717700 & 4.46548700 & -0.77259500 \\ \mathrm{H} & -3.11530600 & 4.84562400 & -1.33182100 \\ \mathrm{O} & -1.72132600 & -0.96178800 & 2.20358500 \\ \mathrm{Cl} & -3.41719800 & 5.31465200 & 0.96531500 \\ \mathrm{C} & -0.93404700 & -1.84397200 & 1.88494000 \\ \mathrm{C} & 0.22190100 & -2.28206100 & 2.75854100 \\ \mathrm{C} & -1.00047000 & -2.55460300 & 0.53517400 \\ \mathrm{C} & 1.57351000 & -1.97813000 & 2.03369000 \\ \mathrm{C} & 0.40531700 & -2.50445800 & -0.20223300 \\ \mathrm{C} & 1.29169400 & -1.51561700 & 0.60236800 \\ \mathrm{H} & 0.14835200 & -1.77536600 & 3.72269300 \\ \mathrm{C} & -2.08420600 & -2.20347700 & -0.49874300 \\ \mathrm{C} & -1.41373800 & -2.29478700 & -1.86110900 \\ \mathrm{H} & 0.13034000 & -3.36038700 & 2.93825400 \\ \mathrm{H} & 2.10612100 & -1.18041600 & 2.55793100 \\ \mathrm{C} & -3.10836000 & -3.27218700 & -0.03127300\end{array}$




$\begin{array}{lrrr}\mathrm{C} & -1.83960600 & -3.89349500 & 0.67918000 \\ \mathrm{H} & -2.01808200 & -4.21692800 & 1.71006900 \\ \mathrm{H} & -1.40278400 & -4.70505900 & 0.09592800 \\ \mathrm{C} & -4.16051500 & -2.82058100 & 0.99267800 \\ \mathrm{H} & -3.74082700 & -2.20096000 & 1.78733000 \\ \mathrm{H} & -4.96788700 & -2.26805000 & 0.49544200 \\ \mathrm{H} & -4.61937700 & -3.69932700 & 1.45726800 \\ \mathrm{C} & -3.77945200 & -4.12358000 & -1.10933300 \\ \mathrm{H} & -4.49840900 & -3.53381900 & -1.69061400 \\ \mathrm{H} & -3.06939600 & -4.57492200 & -1.80735300 \\ \mathrm{H} & -4.33849900 & -4.93948300 & -0.63875600 \\ \mathrm{C} & 0.05115600 & -1.91787900 & -1.60355700 \\ \mathrm{H} & 0.72543500 & -2.28153700 & -2.38211000 \\ \mathrm{C} & 1.08025100 & -3.87937000 & -0.34190500 \\ \mathrm{H} & 2.09681100 & -3.74582700 & -0.72393200 \\ \mathrm{H} & 1.14328700 & -4.41823200 & 0.60894600 \\ \mathrm{H} & 0.54779300 & -4.52043600 & -1.05205300 \\ \mathrm{H} & -2.46123700 & -0.83301500 & -0.32761700 \\ \mathrm{H} & 0.75996100 & -0.55998300 & 0.66531000 \\ \mathrm{H} & 0.15893700 & -0.82850500 & -1.60041400 \\ \mathrm{H} & -1.48375000 & -3.32587300 & -2.23159500 \\ \mathrm{H} & -1.89935700 & -1.68896500 & -2.63688100 \\ \mathrm{H} & 2.22587600 & -2.85438100 & 2.03581000 \\ \mathrm{O} & 2.54337800 & -1.25825800 & -0.05892700 \\ \mathrm{~S} & 2.71345900 & 0.15738700 & -0.93914800 \\ \mathrm{O} & 2.72942100 & -0.21070900 & -2.34948400 \\ \mathrm{O} & 1.66722100 & 1.08492800 & -0.45400100 \\ \mathrm{C} & 4.31420700 & 0.69009300 & -0.41761000 \\ \mathrm{C} & 5.42319600 & 0.32084900 & -1.18486800 \\ \mathrm{C} & 4.44675700 & 1.45640700 & 0.74462800 \\ \mathrm{C} & 6.68612500 & 0.72955300 & -0.76704800 \\ \mathrm{H} & 5.29688300 & -0.26395500 & -2.08928200 \\ \mathrm{C} & 5.71995000 & 1.85319900 & 1.13881500 \\ \mathrm{H} & 3.57325000 & 1.74622500 & 1.31896000 \\ \mathrm{C} & 6.85799800 & 1.49902800 & 0.39450300 \\ \mathrm{H} & 7.55447300 & 0.44818600 & -1.35597200 \\ \mathrm{H} & 5.83564200 & 2.45170600 & 2.03809500 \\ \mathrm{C} & 8.22983700 & 1.95918200 & 0.81644500 \\ \mathrm{H} & 8.99719000 & 1.22539600 & 0.55230200 \\ \mathrm{H} & 8.49166200 & 2.89907500 & 0.31330100 \\ \mathrm{H} & & & 1.89411700\end{array}$




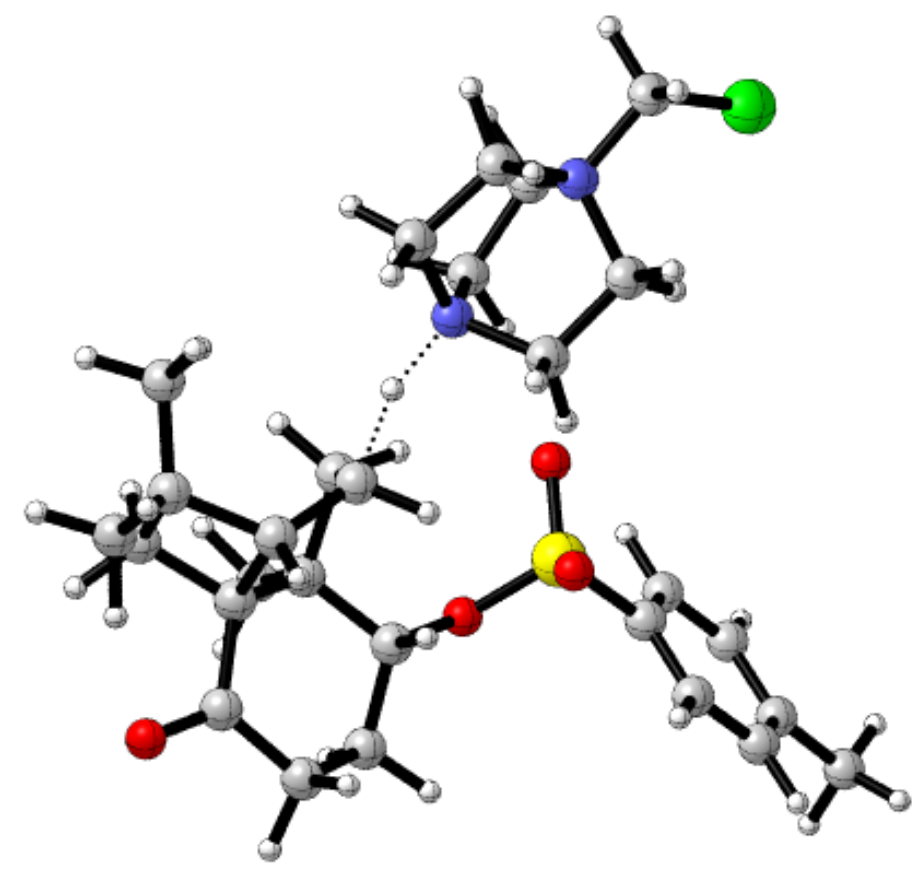

Figure S37. Transition state involving carbon C10 (bottom hydrogen) using SRD.

Zero-point correction $=$

Thermal correction to Energy=

Thermal correction to Enthalpy=

Thermal correction to Gibbs Free Energy=

Sum of electronic and zero-point Energies $=$

Sum of electronic and thermal Energies=

Sum of electronic and thermal Enthalpies $=$

Sum of electronic and thermal Free Energies=
0.672053 (Hartree/Particle)

0.707699

0.708643

0.601795

$-2359.659286$

$-2359.623640$

$-2359.622696$

$-2359.729544$

Number of Imaginary Frequencies $=1$

E (Single Point Energy) $\left[\operatorname{IEFPCM}_{(\mathrm{CH} 3 \mathrm{CN})} \mathrm{U \omega B}\right.$ B7X-D/6-311++G(2d,2p)] $=-2360.62251326$

\section{2}

$\begin{array}{lrrr}\mathrm{C} & -2.52642300 & -1.06209300 & 1.11145500 \\ \mathrm{C} & -4.24677200 & -0.08318700 & -0.32939200 \\ \mathrm{C} & -2.29524500 & -1.17605200 & -1.31450000 \\ \mathrm{C} & -3.46518800 & -2.28927800 & 1.19996200 \\ \mathrm{H} & -2.71360600 & -0.37617700 & 1.93999300 \\ \mathrm{H} & -1.47125700 & -1.34987500 & 1.12949400 \\ \mathrm{C} & -4.97455800 & -1.43230500 & -0.56037000 \\ \mathrm{H} & -4.39854800 & 0.58687600 & -1.17845200 \\ \mathrm{H} & -4.60059800 & 0.42175400 & 0.57165700 \\ \mathrm{H} & -2.60277600 & -0.68860100 & -2.24265100\end{array}$




$\begin{array}{lrrr}\mathrm{H} & -1.20167400 & -1.20803400 & -1.28266900 \\ \mathrm{C} & -2.88624900 & -2.60077500 & -1.18993700 \\ \mathrm{H} & -4.31852600 & -2.12131900 & 1.86135900 \\ \mathrm{H} & -2.93218200 & -3.18086900 & 1.53231100 \\ \mathrm{H} & -5.25354000 & -1.57992800 & -1.60594000 \\ \mathrm{H} & -5.87156700 & -1.51652600 & 0.05699600 \\ \mathrm{H} & -3.28377300 & -2.96323000 & -2.14038900 \\ \mathrm{H} & -2.15581400 & -3.32061200 & -0.82072400 \\ \mathrm{~N} & -2.79056100 & -0.34499600 & -0.17433700 \\ \mathrm{~N} & -4.03502300 & -2.57447700 & -0.18504400 \\ \mathrm{C} & -4.83793600 & -3.87255700 & -0.20422300 \\ \mathrm{H} & -5.29538700 & -3.95327900 & -1.19108600 \\ \mathrm{H} & -5.60316300 & -3.78933200 & 0.56851500 \\ \mathrm{O} & 1.10180900 & 5.65761600 & -0.84285600 \\ \mathrm{Cl} & -3.84739200 & -5.30202700 & 0.10326300 \\ \mathrm{C} & 1.29952800 & 4.47974900 & -0.62731400 \\ \mathrm{C} & 2.54131600 & 3.78632000 & -1.18453600 \\ \mathrm{C} & 0.27839800 & 3.66350100 & 0.19293800 \\ \mathrm{C} & 3.08088400 & 2.61580900 & -0.34179700 \\ \mathrm{C} & 0.88544500 & 2.44289100 & 0.97252400 \\ \mathrm{C} & 1.89885400 & 1.73245300 & 0.02706200 \\ \mathrm{H} & 2.26037500 & 3.41619300 & -2.18304000 \\ \mathrm{C} & -0.97927600 & 3.04915300 & -0.69557100 \\ \mathrm{C} & -0.98193500 & 1.63361400 & -0.24636000 \\ \mathrm{H} & 3.29808000 & 4.55812300 & -1.34738100 \\ \mathrm{H} & 3.80334000 & 2.03987100 & -0.92780300 \\ \mathrm{H} & -2.11131000 & 0.76198200 & -0.19326100 \\ \mathrm{H} & -1.89546900 & 4.14353800 & -0.00428300 \\ \mathrm{C} & -0.74151400 & 4.55155600 & 0.95358000 \\ \mathrm{C} & -0.48793200 & 5.61276100 & 0.93625500 \\ \mathrm{H} & -0.89963300 & 4.24575900 & 1.99120100 \\ \mathrm{H} & -2.24559000 & 5.24736900 & -1.00980700 \\ \mathrm{C} & -1.35181300 & 5.66286100 & -1.48206100 \\ \mathrm{H} & -2.91317100 & 4.87031300 & -1.79434600 \\ \mathrm{H} & -2.75858400 & 6.06843200 & -0.49612100 \\ \mathrm{H} & -3.16802400 & 3.62244900 & 0.66801400 \\ \mathrm{H} & -3.87658900 & 3.23947400 & -0.07930400 \\ \mathrm{H} & -2.96898400 & 2.83456500 & 1.40459300 \\ \mathrm{H} & -3.66873200 & 4.43967400 & 1.19897300 \\ \mathrm{H} & 3.60536700 & 2.97661900 & 0.54743600 \\ \mathrm{H} & -1.01086900 & 1.95569400 & 1.90992300 \\ \mathrm{H} & 2.00504700 & 1.94418400 & 2.76734800 \\ \mathrm{H} & & & \\ \mathrm{H} & & & \\ \mathrm{H} & & & \\ \mathrm{H} & & & \end{array}$




$\begin{array}{lrrr}\mathrm{H} & -0.92376100 & 3.15066200 & -1.78227200 \\ \mathrm{H} & -0.53889500 & 0.95544900 & -0.98512600 \\ \mathrm{H} & 1.40766400 & 1.45486900 & -0.90726500 \\ \mathrm{H} & -0.09130400 & 0.51981700 & 1.44773400 \\ \mathrm{O} & 2.37022200 & 0.48430500 & 0.63381600 \\ \mathrm{~S} & 1.78726100 & -0.96529300 & 0.10571600 \\ \mathrm{O} & 0.91092800 & -1.51121500 & 1.15786200 \\ \mathrm{O} & 1.16731200 & -0.76607200 & -1.22144800 \\ \mathrm{C} & 3.26370800 & -1.92236000 & -0.01168300 \\ \mathrm{C} & 3.72699100 & -2.60040600 & 1.12061800 \\ \mathrm{C} & 3.95100800 & -1.96409200 & -1.22953300 \\ \mathrm{C} & 4.90674700 & -3.33061000 & 1.01918500 \\ \mathrm{H} & 3.17319900 & -2.56524100 & 2.05240700 \\ \mathrm{C} & 5.12587600 & -2.70497100 & -1.30289300 \\ \mathrm{H} & 3.56653500 & -1.44529200 & -2.10116900 \\ \mathrm{C} & 5.62755200 & -3.39244500 & -0.18491900 \\ \mathrm{H} & 5.27435300 & -3.86534200 & 1.89031200 \\ \mathrm{H} & 5.66281200 & -2.75424800 & -2.24590600 \\ \mathrm{C} & 6.92002900 & -4.16242300 & -0.27040500 \\ \mathrm{H} & 6.93088400 & -5.00854100 & 0.42280100 \\ \mathrm{H} & 7.09723600 & -4.54063400 & -1.28138800 \\ \mathrm{H} & 7.76824300 & -3.51604100 & -0.00978200\end{array}$

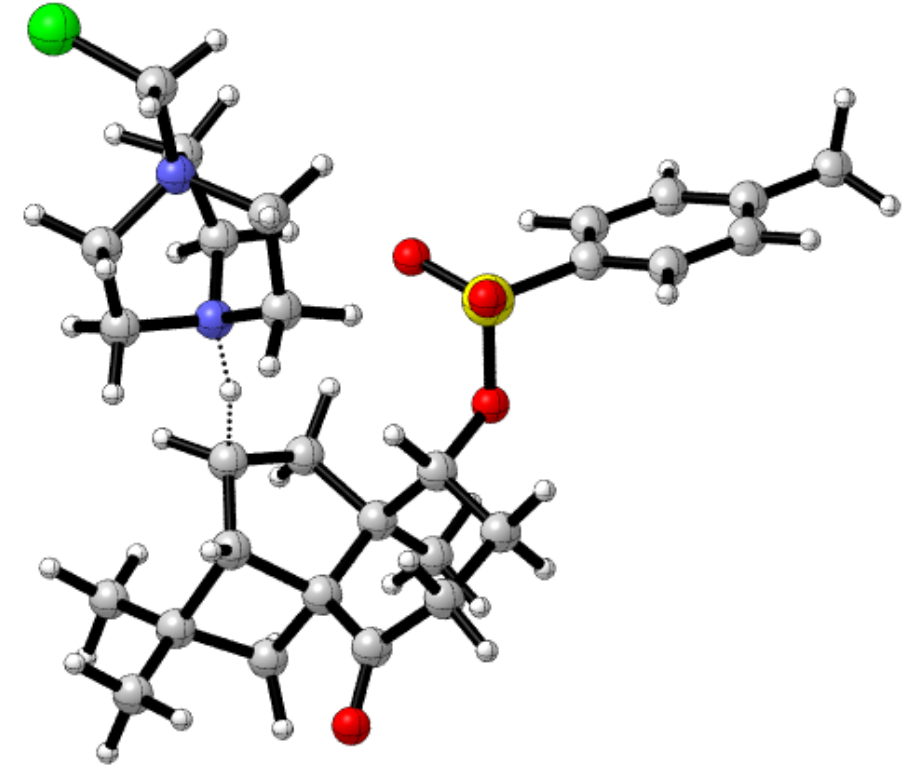

Figure S38. Transition state involving carbon C10 (top hydrogen) using SRD.

Zero-point correction $=$

Thermal correction to Energy=

Thermal correction to Enthalpy=
0.670954 (Hartree/Particle)

0.706620

0.707564 
Thermal correction to Gibbs Free Energy=

Sum of electronic and zero-point Energies $=$

Sum of electronic and thermal Energies=

Sum of electronic and thermal Enthalpies=

Sum of electronic and thermal Free Energies=
0.600602

$-2359.673535$

$-2359.637870$

$-2359.636926$

$-2359.743887$

Number of Imaginary Frequencies $=1$

E (Single Point Energy) IEFPCM $_{(\mathrm{CH} 3 \mathrm{CN})} \mathrm{U \omega B}$ B7X-D/6-311++G(2d,2p)] = -2360.63295839

22

$\begin{array}{lrrr}\mathrm{C} & -3.64081000 & 1.12163400 & -0.04884700 \\ \mathrm{C} & -1.57502700 & 1.37417900 & 1.21396000 \\ \mathrm{C} & -1.63084500 & 1.92035200 & -1.17574800 \\ \mathrm{C} & -4.01981600 & 2.51735600 & 0.50703700 \\ \mathrm{H} & -4.03075700 & 0.33278700 & 0.59846600 \\ \mathrm{H} & -4.03752600 & 0.96856500 & -1.05485800 \\ \mathrm{C} & -1.76167200 & 2.89262500 & 1.44079700 \\ \mathrm{H} & -0.50760000 & 1.13708400 & 1.23488900 \\ \mathrm{H} & -2.08984200 & 0.78625500 & 1.97724600 \\ \mathrm{H} & -0.53866800 & 1.88530300 & -1.14133800 \\ \mathrm{H} & -1.95681300 & 1.53626100 & -2.14517200 \\ \mathrm{C} & -2.18900400 & 3.34628300 & -0.94667800 \\ \mathrm{H} & -4.33324800 & 2.47697300 & 1.55271600 \\ \mathrm{H} & -4.81108100 & 2.98771700 & -0.07777800 \\ \mathrm{H} & -0.83495900 & 3.44276200 & 1.26326300 \\ \mathrm{H} & -2.11642000 & 3.11133200 & 2.45039600 \\ \mathrm{H} & -1.40044300 & 4.09975800 & -1.00389700 \\ \mathrm{H} & -2.97634200 & 3.61397100 & -1.65155700 \\ \mathrm{~N} & -2.15570000 & 1.00990400 & -0.11221100 \\ \mathrm{~N} & -2.79586600 & 3.42180900 & 0.45054100 \\ \mathrm{C} & -3.14079000 & 4.85437300 & 0.84394500 \\ \mathrm{H} & -2.20422600 & 5.41232300 & 0.87465600 \\ \mathrm{H} & -3.59799300 & 4.81648400 & 1.83339800 \\ \mathrm{O} & -1.16244400 & -4.85910400 & 1.99405900 \\ \mathrm{Cl} & -4.25378600 & 5.63433500 & -0.28567200 \\ \mathrm{C} & -0.61480100 & -3.85818500 & 1.57819400 \\ \mathrm{C} & 0.21480400 & -2.98429400 & 2.50687300 \\ \mathrm{C} & -0.80406100 & -3.41227700 & 0.11365200 \\ \mathrm{C} & 1.42846500 & -2.34476800 & 1.82274800 \\ \mathrm{C} & 0.38406900 & -2.60928900 & -0.52937600 \\ \mathrm{C} & 0.97565300 & -1.65679000 & 0.54325400 \\ \mathrm{H} & -0.45174900 & -2.19295600 & 2.88740400 \\ \mathrm{C} & -2.10518300 & -2.55892100 & -0.14839800 \\ \mathrm{C} & -1.72491400 & -1.49202200 & -1.11143700 \\ \mathrm{H} & 0.49850500 & -3.58927800 & 3.37169900 \\ \mathrm{H} & 1.89088300 & -1.60627800 & 2.48423900 \\ & & & \\ & & & \end{array}$




$\begin{array}{lccc}\mathrm{H} & -1.84851700 & -0.22074500 & -0.45706200 \\ \mathrm{C} & -2.80735600 & -3.87510100 & -0.86313100 \\ \mathrm{C} & -1.41523300 & -4.55340000 & -0.75658100 \\ \mathrm{H} & -1.43232300 & -5.51463700 & -0.23778000 \\ \mathrm{H} & -0.93205900 & -4.67185400 & -1.72822100 \\ \mathrm{C} & -3.86479800 & -4.45562800 & 0.07510000 \\ \mathrm{H} & -3.47615000 & -4.61919700 & 1.08300100 \\ \mathrm{H} & -4.74855400 & -3.80828000 & 0.12814900 \\ \mathrm{H} & -4.19343400 & -5.42750500 & -0.31348800 \\ \mathrm{C} & -3.36119100 & -3.70795400 & -2.27216200 \\ \mathrm{H} & -4.25317300 & -3.07000200 & -2.28999100 \\ \mathrm{H} & -2.62809900 & -3.30653000 & -2.97985200 \\ \mathrm{H} & -3.66509900 & -4.69139600 & -2.64963700 \\ \mathrm{H} & 2.19100600 & -3.09719500 & 1.59740000 \\ \mathrm{C} & -0.32057300 & -1.75419700 & -1.62233200 \\ \mathrm{H} & -0.40021700 & -2.32148700 & -2.56364400 \\ \mathrm{C} & 1.48351800 & -3.51133300 & -1.11672200 \\ \mathrm{H} & 2.30429400 & -2.90861000 & -1.51356300 \\ \mathrm{H} & 1.89804000 & -4.19277900 & -0.36729700 \\ \mathrm{H} & 1.09777300 & -4.12448900 & -1.93512500 \\ \mathrm{H} & -2.71712900 & -2.25347500 & 0.70847200 \\ \mathrm{H} & -2.47277500 & -1.20612500 & -1.86234600 \\ \mathrm{H} & 0.21571200 & -0.91345100 & 0.81226500 \\ \mathrm{H} & 0.24436800 & -0.84978300 & -1.87755700 \\ \mathrm{O} & 2.11761400 & -0.94614600 & -0.04323700 \\ \mathrm{~S} & 2.13652200 & 0.68966900 & 0.03159800 \\ \mathrm{O} & 1.40751700 & 1.19950400 & -1.14740300 \\ \mathrm{O} & 1.60073300 & 1.09716400 & 1.34571200 \\ \mathrm{C} & 3.85830200 & 1.02051700 & -0.10611900 \\ \mathrm{C} & 4.63372800 & 1.07363700 & 1.05827600 \\ \mathrm{C} & 4.41740200 & 1.20227700 & -1.37558800 \\ \mathrm{C} & 5.99699500 & 1.31502800 & 0.93472400 \\ \mathrm{H} & 4.17981000 & 0.93914100 & 2.03419100 \\ \mathrm{C} & 5.78483400 & 1.44015700 & -1.46869300 \\ \mathrm{H} & 3.79756700 & 1.16421500 & -2.26465500 \\ \mathrm{H} & 6.59579500 & 1.50101800 & -0.32347000 \\ \mathrm{H} & 6.60993300 & 1.36078100 & 1.83035600 \\ \mathrm{H} & 6.23185500 & 1.58200000 & -2.44836600 \\ \mathrm{H} & 8.07087200 & 1.78580900 & -0.43653300 \\ \mathrm{H} & 8.26388000 & 2.86024000 & -0.31996400 \\ \mathrm{H} & & 1.26780400 & 0.34139400\end{array}$




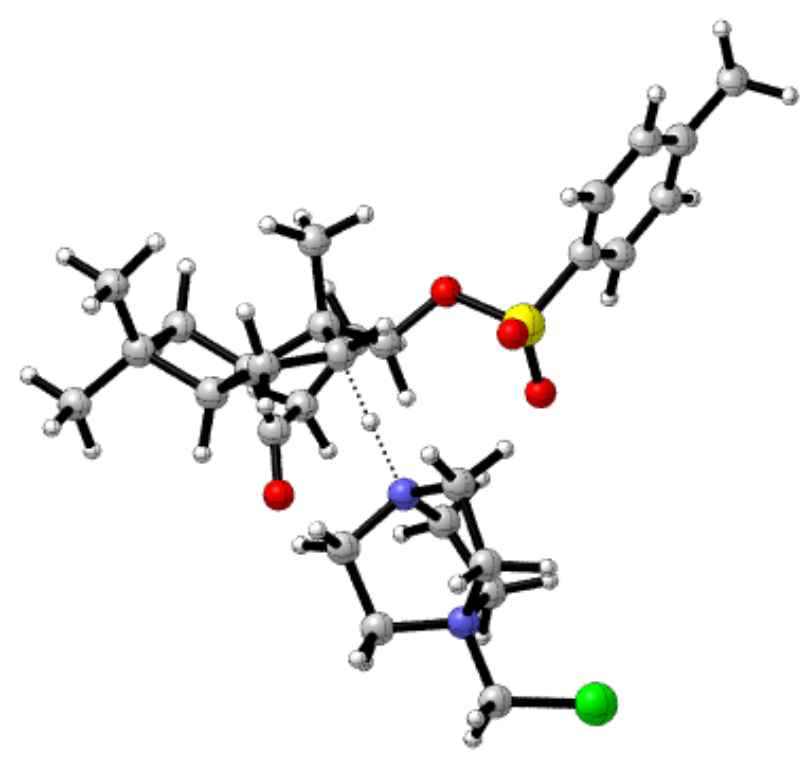

Figure S39. Transition state involving carbon C26 (top hydrogen) using SRD.

Zero-point correction $=$

Thermal correction to Energy=

Thermal correction to Enthalpy=

Thermal correction to Gibbs Free Energy=

Sum of electronic and zero-point Energies $=$

Sum of electronic and thermal Energies=

Sum of electronic and thermal Enthalpies=

Sum of electronic and thermal Free Energies=
0.671805 (Hartree/Particle)

0.707081

0.708025

0.603582

$-2359.670234$

$-2359.634958$

$-2359.634014$

$-2359.738457$

Number of Imaginary Frequencies $=1$

E (Single Point Energy) $\left[\operatorname{IEFPCM}_{(\mathrm{CH} 3 \mathrm{CN})} \mathrm{U} \omega \mathrm{B} 97 \mathrm{X}-\mathrm{D} / 6-311++\mathrm{G}(2 \mathrm{~d}, 2 \mathrm{p})\right]=-2360.62067530$

22

$\mathrm{C}$

$\mathrm{C}$

$-1.36671700$

2.02485000

$-1.56461600$

C $\quad-3.26176400$

$0.94855100-0.44937000$

$\mathrm{C}$

$-1.34383400$

$1.66999200 \quad 0.85506300$

C

$-2.19757900$

$3.33134100-1.47711400$

$\mathrm{H}$

$-1.54971600$

$1.52513600-2.51864800$

$\mathrm{H}$

$-0.29233900$

$2.19297200-1.46764600$

$\mathrm{C}$

$-3.91283900$

$2.29098500-0.02385300$

$\mathrm{H}$

$-3.50166000$

$\begin{array}{lll}0.15314600 & 0.26143800\end{array}$

$\mathrm{H}$

$-3.58208300$

$0.65366000-1.45029800$

$\mathrm{H}$

$-1.78074100$

1.04167500

1.63353500

$\mathrm{H}$

$-0.25401200$

1.63165700

0.92571600

C

$-1.81787000$

3.14206100

0.96172500 


\begin{tabular}{|c|c|c|c|}
\hline $\mathrm{H}$ & -2.99608300 & 3.37068900 & -2.22180800 \\
\hline $\mathrm{H}$ & -1.57111200 & 4.21737500 & -1.58569400 \\
\hline $\mathrm{H}$ & -4.26634900 & 2.26727300 & 1.00915400 \\
\hline $\mathrm{H}$ & -4.74656700 & 2.55896700 & -0.67653300 \\
\hline $\mathrm{H}$ & -2.27233100 & 3.35104200 & 1.93264000 \\
\hline $\mathrm{H}$ & -1.00745600 & 3.85179200 & 0.79559300 \\
\hline $\mathrm{N}$ & -1.78657100 & 1.12312500 & -0.46006700 \\
\hline $\mathrm{N}$ & -2.86970100 & 3.40171700 & -0.11065400 \\
\hline $\mathrm{C}$ & -3.58006400 & 4.73480800 & 0.09508300 \\
\hline $\mathrm{H}$ & -4.11457100 & 4.67135100 & 1.04362700 \\
\hline $\mathrm{H}$ & -4.27940600 & 4.85984600 & -0.73246900 \\
\hline $\mathrm{O}$ & -2.57875300 & -1.22152900 & 1.85122800 \\
\hline $\mathrm{Cl}$ & -2.47170100 & 6.11050600 & 0.13749200 \\
\hline $\mathrm{C}$ & -1.70322300 & -2.07415500 & 1.74327900 \\
\hline $\mathrm{C}$ & -0.67671700 & -2.32835200 & 2.83166100 \\
\hline $\mathrm{C}$ & -1.45217700 & -2.80961700 & 0.43903300 \\
\hline $\mathrm{C}$ & 0.74875400 & -2.25893700 & 2.20816600 \\
\hline $\mathrm{C}$ & -0.14081100 & -2.15996500 & -0.18505300 \\
\hline $\mathrm{C}$ & 0.67601900 & -1.42357000 & 0.92456800 \\
\hline $\mathrm{H}$ & -0.81628700 & -1.58109600 & 3.61713700 \\
\hline $\mathrm{C}$ & -2.56721900 & -2.71434100 & -0.63791200 \\
\hline $\mathrm{C}$ & -1.94841200 & -1.98269800 & -1.84549000 \\
\hline $\mathrm{H}$ & -0.84820400 & -3.31373900 & 3.28031400 \\
\hline $\mathrm{H}$ & 1.44253600 & -1.78366500 & 2.90592800 \\
\hline $\mathrm{H}$ & -2.64362300 & -1.31940500 & -2.37485600 \\
\hline $\mathrm{C}$ & -2.76288200 & -4.28047600 & -0.58784200 \\
\hline $\mathrm{C}$ & -1.62563200 & -4.36629000 & 0.48935700 \\
\hline $\mathrm{H}$ & -1.96431000 & -4.74239100 & 1.45989400 \\
\hline $\mathrm{H}$ & -0.74734400 & -4.94405300 & 0.19403400 \\
\hline $\mathrm{C}$ & -4.13799500 & -4.65510600 & -0.02299000 \\
\hline $\mathrm{H}$ & -4.35952200 & -4.10754700 & 0.90119100 \\
\hline $\mathrm{H}$ & -4.93227700 & -4.43154300 & -0.74513400 \\
\hline $\mathrm{H}$ & -4.18609700 & -5.72571100 & 0.20439600 \\
\hline $\mathrm{C}$ & -2.49472000 & -5.06280300 & -1.87373700 \\
\hline $\mathrm{H}$ & -3.24328100 & -4.83288900 & -2.64114700 \\
\hline $\mathrm{H}$ & -1.50380200 & -4.87137000 & -2.30172500 \\
\hline $\mathrm{H}$ & -2.55017500 & -6.13874900 & -1.67483700 \\
\hline $\mathrm{H}$ & 1.14176000 & -3.25663700 & 1.99322000 \\
\hline $\mathrm{C}$ & -0.72791800 & -1.27745000 & -1.28442500 \\
\hline $\mathrm{H}$ & -0.01643000 & -0.81058900 & -1.97328100 \\
\hline $\mathrm{C}$ & 0.79408100 & -3.17950000 & -0.90252200 \\
\hline $\mathrm{H}$ & 1.24210800 & -3.85800300 & -0.17046700 \\
\hline $\mathrm{H}$ & 0.25059500 & -3.77946100 & -1.63374100 \\
\hline $\mathrm{H}$ & 1.60448500 & -2.65563900 & -1.41175600 \\
\hline $\mathrm{H}$ & -3.45867100 & -2.18910700 & -0.28409400 \\
\hline $\mathrm{H}$ & -1.59377500 & -2.67897400 & -2.6232430 \\
\hline
\end{tabular}




$\begin{array}{lrrr}\mathrm{H} & -1.19808800 & -0.16259100 & -0.74712100 \\ \mathrm{H} & 0.17535700 & -0.48842500 & 1.18780000 \\ \mathrm{O} & 2.01428000 & -1.09312100 & 0.47547900 \\ \mathrm{~S} & 2.28821000 & 0.50497600 & 0.12781500 \\ \mathrm{O} & 1.60537900 & 0.80262000 & -1.14708000 \\ \mathrm{O} & 1.85824900 & 1.28917500 & 1.29659400 \\ \mathrm{C} & 4.03482500 & 0.50045600 & -0.05751300 \\ \mathrm{C} & 4.58611600 & 0.29800200 & -1.32750700 \\ \mathrm{C} & 4.83480000 & 0.69436100 & 1.07526300 \\ \mathrm{C} & 5.97109400 & 0.28710300 & -1.45320000 \\ \mathrm{H} & 3.94776800 & 0.16284300 & -2.19375100 \\ \mathrm{C} & 6.21572800 & 0.67860800 & 0.91908300 \\ \mathrm{H} & 4.38683200 & 0.86213900 & 2.04870100 \\ \mathrm{C} & 6.80725800 & 0.47611800 & -0.34005400 \\ \mathrm{H} & 6.41295700 & 0.13213700 & -2.43321400 \\ \mathrm{H} & 6.84855300 & 0.82893000 & 1.78900100 \\ \mathrm{C} & 8.30556700 & 0.49097600 & -0.49474500 \\ \mathrm{H} & 8.66646800 & 1.51975100 & -0.62382000 \\ \mathrm{H} & 8.80378400 & 0.08107600 & 0.38887800 \\ \mathrm{H} & 8.62611900 & -0.08082000 & -1.37006500\end{array}$

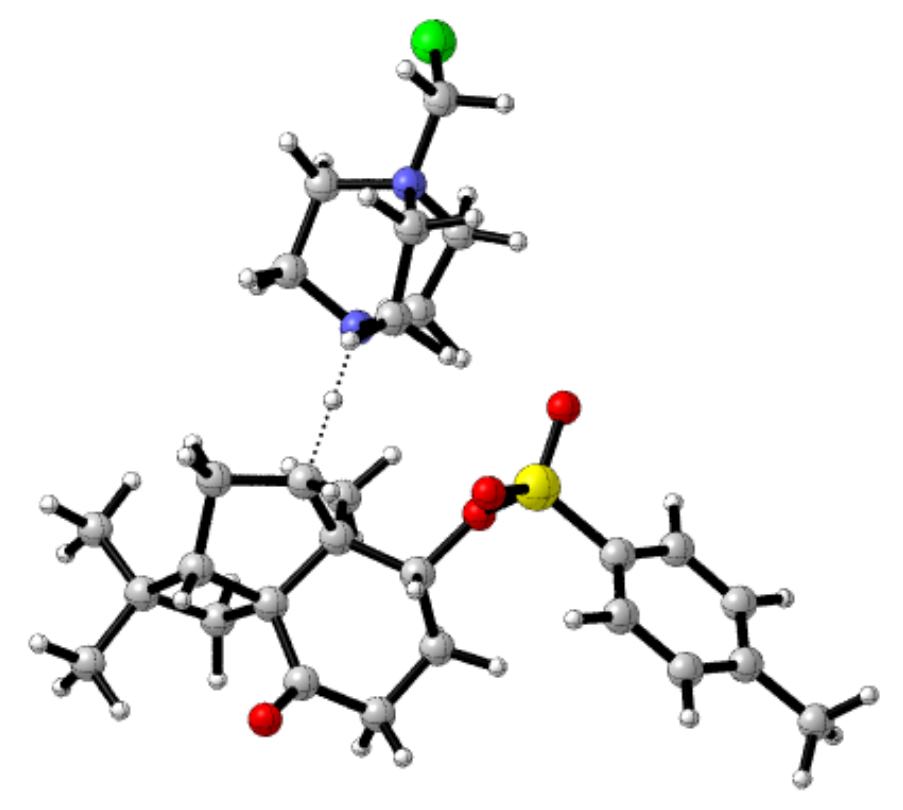

Figure S40. Transition state involving carbon C26 (bottom hydrogen) using SRD.

Zero-point correction $=$

Thermal correction to Energy=

Thermal correction to Enthalpy=

Thermal correction to Gibbs Free Energy=
0.671698 (Hartree/Particle)

0.707399

0.708344

0.601230 
Sum of electronic and zero-point Energies=

Sum of electronic and thermal Energies=

Sum of electronic and thermal Enthalpies=

Sum of electronic and thermal Free Energies=
$-2359.664011$

$-2359.628309$

$-2359.627365$

$-2359.734478$

Number of Imaginary Frequencies $=1$

E (Single Point Energy) $\left[\operatorname{IEFPCM}_{(\mathrm{CH} 3 \mathrm{CN})} \mathrm{U \omega B} 97 \mathrm{X}-\mathrm{D} / 6-311++\mathrm{G}(2 \mathrm{~d}, 2 \mathrm{p})\right]=-2360.62432526$

22

$\mathrm{C}$

$\begin{array}{lll}3.68965900 & 1.12663300 & 0.39330800\end{array}$

$\begin{array}{lll}2.43069100 & -0.61240700 & 1.54981300\end{array}$

$\begin{array}{lll}2.51594100 & -0.56458500 & -0.90805200\end{array}$

$\begin{array}{llll}4.94260200 & 0.23603300 & 0.18472900\end{array}$

$\begin{array}{lll}3.70639000 & 1.63355800 & 1.36046400\end{array}$

$\begin{array}{lll}3.62394400 & 1.88337300 & -0.39109800\end{array}$

$\begin{array}{lll}3.78883300 & -1.33882200 & 1.69409400\end{array}$

$\begin{array}{llll}\mathrm{H} & 1.60092400 & -1.30947700 & 1.43756000\end{array}$

$\mathrm{H} \quad 2.23545100 \quad 0.01478400 \quad 2.42224000$

$\mathrm{H} \quad \begin{array}{llll}1.54762700 & -1.04711800 & -1.04783400\end{array}$

$\begin{array}{llll}\mathrm{H} & 2.71929000 & 0.10668100 & -1.74458000\end{array}$

$\begin{array}{llll}\mathrm{C} & 3.60667700 & -1.64207900 & -0.74674100\end{array}$

$\begin{array}{llll}\mathrm{H} & 5.72966600 & 0.46865700 & 0.90525500\end{array}$

$\mathrm{H} \quad \begin{array}{llll}\mathrm{H} & 5.35590700 & 0.32646400 & -0.81962700\end{array}$

$\begin{array}{llll}\mathrm{H} & 3.64769500 & -2.39985800 & 1.91095000\end{array}$

$\begin{array}{llll}\mathrm{H} & 4.42247300 & -0.90135200 & 2.46901200\end{array}$

$\mathrm{H} \quad 3.17508200 \quad-2.60432900-0.46095600$

$\mathrm{H} \quad 4.19859700 \quad-1.76605300 \quad-1.65427700$

$\begin{array}{llll}\mathrm{N} & 2.47558300 & 0.26370800 & 0.33763900\end{array}$

$\begin{array}{llll}\mathrm{N} & 4.55071100 & -1.22481300 & 0.37819700\end{array}$

$\begin{array}{llll}\mathrm{C} & 5.76982200 & -2.13727500 & 0.45356400\end{array}$

$\begin{array}{llll}\mathrm{H} & 5.39963200 & -3.15964600 & 0.53810900\end{array}$

$\mathrm{H} \quad \begin{array}{llll}\mathrm{H} & 6.33088400 & -1.85591400 & 1.34556200\end{array}$

$\begin{array}{llll}\mathrm{O} & -3.45177000 & 3.29192400 & 1.35598300\end{array}$

$\mathrm{Cl} \quad \begin{array}{llll}6.81728900 & -2.00829600 & -0.96400800\end{array}$

$\begin{array}{llll}\text { C } & -3.03532900 & 2.75851600 & 0.34963600\end{array}$

$\begin{array}{llll}\text { C } & -3.90075300 & 1.81197900 & -0.47924100\end{array}$

$\begin{array}{llll}\mathrm{C} & -1.62406700 & 3.04814400 & -0.17795300\end{array}$

$\begin{array}{llll}\text { C } & -3.08707700 & 0.65949600 & -1.11553800\end{array}$

$\begin{array}{llll}\text { C } & -0.83271200 & 1.70683700 & -0.51373700\end{array}$

$\begin{array}{llll}\text { C } & -1.78134900 & 0.49262900 & -0.33840400\end{array}$

$\begin{array}{llll}\mathrm{H} & -4.68832500 & 1.44143500 & 0.18239700\end{array}$

$\begin{array}{llll}\mathrm{C} & -0.73406800 & 3.90827300 & 0.78177200\end{array}$

$\begin{array}{llll}\mathrm{C} & 0.63667200 & 3.18350100 & 0.87998900\end{array}$

$\mathrm{H} \quad-4.39983300 \quad 2.40690000 \quad-1.25470100$

$\mathrm{H} \quad \begin{array}{llll}\mathrm{H} & -3.65743100 & -0.27237000 & -1.06854700\end{array}$

$\begin{array}{lllll}\text { C } & -0.90078900 & 5.15901900 & -0.17341000\end{array}$ 


$\begin{array}{lrrr}\mathrm{C} & -1.66280700 & 4.25022300 & -1.19393500 \\ \mathrm{H} & -2.67103100 & 4.58768800 & -1.45521500 \\ \mathrm{C} & -1.79607000 & 6.22131500 & 0.47840900 \\ \mathrm{H} & -2.70298800 & 5.78253600 & 0.90407300 \\ \mathrm{H} & -1.26192500 & 6.73898900 & 1.28357100 \\ \mathrm{H} & -2.09230400 & 6.97257500 & -0.26331500 \\ \mathrm{C} & 0.36652300 & 5.80717900 & -0.72668500 \\ \mathrm{H} & 0.96401700 & 6.26375600 & 0.07135400 \\ \mathrm{H} & 1.00469100 & 5.10313900 & -1.27597400 \\ \mathrm{H} & 0.09934300 & 6.60280900 & -1.43138000 \\ \mathrm{H} & -2.87511600 & 0.84683400 & -2.17226800 \\ \mathrm{C} & 0.21469500 & 1.75331000 & 0.61078500 \\ \mathrm{H} & -0.07270800 & 1.18191200 & 1.50307600 \\ \mathrm{C} & -0.19725100 & 1.72193400 & -1.91854600 \\ \mathrm{H} & 0.27796900 & 0.76345400 & -2.14034500 \\ \mathrm{H} & -0.94510300 & 1.89704400 & -2.69605600 \\ \mathrm{H} & 0.55107600 & 2.51573300 & -2.00759600 \\ \mathrm{H} & -2.01172200 & 0.38018700 & 0.72662400 \\ \mathrm{H} & 1.40744500 & 0.98467500 & 0.35320600 \\ \mathrm{H} & 1.32537600 & 3.56115200 & 0.11521300 \\ \mathrm{H} & -1.17282200 & 4.04006700 & 1.77383800 \\ \mathrm{H} & -1.11934000 & 4.07715400 & -2.12528500 \\ \mathrm{H} & 1.11430600 & 3.31048100 & 1.85571900 \\ \mathrm{O} & -1.06897400 & -0.71043600 & -0.76589300 \\ \mathrm{~S} & -0.88266600 & -1.97475200 & 0.28499500 \\ \mathrm{O} & 0.28691400 & -2.66057900 & -0.28598300 \\ \mathrm{O} & -0.76825300 & -1.41291700 & 1.63848400 \\ \mathrm{C} & -2.32858000 & -2.97271100 & 0.14574500 \\ \mathrm{C} & -2.49592400 & -3.77758000 & -0.98903800 \\ \mathrm{C} & -3.28141700 & -2.93439200 & 1.16998700 \\ \mathrm{C} & -3.64483200 & -4.55236600 & -1.08758500 \\ \mathrm{H} & -1.74037800 & -3.80543600 & -1.76702300 \\ \mathrm{C} & -4.42201300 & -3.72273700 & 1.04442100 \\ \mathrm{H} & -3.12383600 & -2.31972800 & 2.04947200 \\ \mathrm{H} & -4.62449500 & -4.54174000 & -0.07776900 \\ \mathrm{H} & -3.78476800 & -5.18324900 & -1.96076300 \\ & -5.16494400 & -3.70731000 & 1.83659100 \\ \mathrm{H} & -5.84718300 & -5.41471200 & -0.18771400 \\ \mathrm{H} & -6.66893600 & -5.03785000 & 0.42729600 \\ \mathrm{H} & -6.19427300 & -5.48980700 & -1.22281000\end{array}$




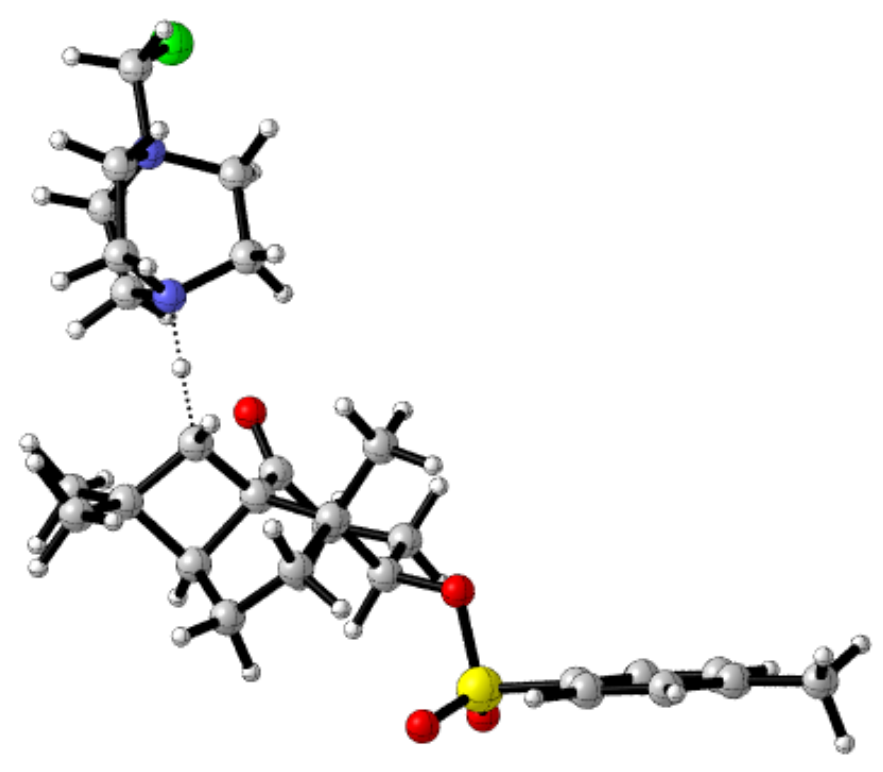

Figure S41. Transition state involving carbon C14 (top hydrogen) using SRD.

Zero-point correction $=$

Thermal correction to Energy=

Thermal correction to Enthalpy=

Thermal correction to Gibbs Free Energy=

Sum of electronic and zero-point Energies=

Sum of electronic and thermal Energies $=$

Sum of electronic and thermal Enthalpies=

Sum of electronic and thermal Free Energies=
0.671533 (Hartree/Particle)

0.707195

0.708139

0.600905

$-2359.666999$

$-2359.631336$

$-2359.630392$

$-2359.737626$

Number of Imaginary Frequencies $=1$

E (Single Point Energy) $\left[\operatorname{IEFPCM}_{(\mathrm{CH} 3 \mathrm{CN})} \mathrm{U} \omega \mathrm{B} 97 \mathrm{X}-\mathrm{D} / 6-311++\mathrm{G}(2 \mathrm{~d}, 2 \mathrm{p})\right]=-2360.63416875$

22

$\mathrm{C}$

C

$\mathrm{C}$

$\mathrm{C}$

$\mathrm{H}$

$\mathrm{H}$

C

$\mathrm{H}$

$\mathrm{H}$

$\mathrm{H}$

$\mathrm{H}$

C

$\mathrm{H}$

$\begin{array}{rrr}-4.71597700 & 0.45436200 & 0.68940700 \\ -4.54488400 & 0.35848000 & -1.74636100 \\ -3.47631500 & -1.35631800 & -0.37771700 \\ -6.09379700 & -0.22566800 & 0.50432800 \\ -4.82238300 & 1.53969500 & 0.71641600 \\ -4.20495100 & 0.13975900 & 1.59785500 \\ -5.71350100 & -0.64725100 & -1.89961100 \\ -3.83709900 & 0.25213100 & -2.57129300 \\ -4.90005900 & 1.39095800 & -1.72975300 \\ -2.92862800 & -1.62242200 & -1.28470200 \\ -2.81848100 & -1.47855300 & 0.48249900 \\ -4.76902600 & -2.19272400 & -0.21929600 \\ -6.86258400 & 0.46655000 & 0.15244000\end{array}$




\begin{tabular}{|c|c|c|c|}
\hline $\mathrm{H}$ & -6.44055900 & -0.69413500 & 1.42620300 \\
\hline $\mathrm{H}$ & -5.48605700 & -1.44229800 & -2.61307000 \\
\hline $\mathrm{H}$ & -6.63427200 & -0.14982100 & -2.21193600 \\
\hline $\mathrm{H}$ & -4.78657700 & -3.05143400 & -0.89400800 \\
\hline $\mathrm{H}$ & -4.91019400 & -2.55080900 & 0.80041100 \\
\hline $\mathrm{N}$ & -3.83609100 & 0.09036300 & -0.46332200 \\
\hline $\mathrm{N}$ & -5.97180500 & -1.31618800 & -0.55428300 \\
\hline $\mathrm{C}$ & -7.25737900 & -2.13246800 & -0.66914600 \\
\hline $\mathrm{H}$ & -7.13029700 & -2.81612900 & -1.50952100 \\
\hline $\mathrm{H}$ & -8.06806100 & -1.42942500 & -0.86465800 \\
\hline $\mathrm{O}$ & -1.96716200 & 0.20352300 & 1.77475200 \\
\hline $\mathrm{Cl}$ & -7.62117300 & -3.05615700 & 0.79112200 \\
\hline $\mathrm{C}$ & -0.81443700 & 0.60048900 & 1.61205600 \\
\hline $\mathrm{C}$ & 0.24604100 & 0.43621000 & 2.66753100 \\
\hline $\mathrm{C}$ & -0.38808800 & 1.21623500 & 0.29023000 \\
\hline $\mathrm{C}$ & 1.46918200 & -0.29428100 & 2.07354800 \\
\hline $\mathrm{C}$ & 0.92902800 & 0.59837800 & -0.30980100 \\
\hline $\mathrm{C}$ & 1.97186300 & 0.46314000 & 0.84669400 \\
\hline $\mathrm{H}$ & 0.55913200 & 1.43200500 & 3.01465600 \\
\hline $\mathrm{C}$ & -0.20683100 & 2.79818200 & 0.24483600 \\
\hline $\mathrm{C}$ & 1.06904700 & 3.03956000 & -0.58232400 \\
\hline $\mathrm{H}$ & -0.18223600 & -0.09430600 & 3.52118800 \\
\hline $\mathrm{H}$ & 2.27207800 & -0.33201000 & 2.81412100 \\
\hline $\mathrm{C}$ & -1.61590900 & 2.98975200 & -0.49161900 \\
\hline $\mathrm{C}$ & -1.51539600 & 1.47697300 & -0.71852000 \\
\hline $\mathrm{H}$ & -2.74813800 & 0.76882700 & -0.43441100 \\
\hline $\mathrm{H}$ & -1.32261900 & 1.16335800 & -1.75049500 \\
\hline $\mathrm{C}$ & -2.65800500 & 3.47755300 & 0.52618000 \\
\hline $\mathrm{H}$ & -2.67744400 & 2.86273400 & 1.43115800 \\
\hline $\mathrm{H}$ & -2.43258600 & 4.50669700 & 0.82406100 \\
\hline $\mathrm{H}$ & -3.66180500 & 3.48981900 & 0.08044700 \\
\hline $\mathrm{C}$ & -1.67159600 & 3.83203900 & -1.76364900 \\
\hline $\mathrm{H}$ & -1.46723600 & 4.88474400 & -1.53766300 \\
\hline $\mathrm{H}$ & -0.95179400 & 3.50320700 & -2.51831800 \\
\hline $\mathrm{H}$ & -2.67258300 & 3.78965500 & -2.21100400 \\
\hline $\mathrm{H}$ & 1.21500700 & -1.32854300 & 1.81524600 \\
\hline $\mathrm{C}$ & 1.39908400 & 1.71041100 & -1.28500800 \\
\hline $\mathrm{H}$ & 0.85915700 & 1.62604600 & -2.23748200 \\
\hline $\mathrm{C}$ & 0.70245000 & -0.75286200 & -1.00135500 \\
\hline $\mathrm{H}$ & 1.64446400 & -1.12426400 & -1.41133800 \\
\hline $\mathrm{H}$ & 0.32211000 & -1.51678100 & -0.31282200 \\
\hline $\mathrm{H}$ & -0.00354600 & -0.65912600 & -1.83547400 \\
\hline $\mathrm{H}$ & 2.24767000 & 1.47113200 & 1.17570600 \\
\hline $\mathrm{H}$ & 2.46444800 & 1.62331100 & -1.51083000 \\
\hline $\mathrm{H}$ & 0.96604500 & 3.87605300 & -1.27862800 \\
\hline $\mathrm{H}$ & 1.87910600 & 3.31389900 & 0.10156600 \\
\hline
\end{tabular}




$\begin{array}{lccc}\mathrm{H} & -0.21260000 & 3.30272600 & 1.21748100 \\ \mathrm{O} & 3.14051900 & -0.18925900 & 0.30919300 \\ \mathrm{~S} & 4.58008100 & 0.62281000 & 0.65092000 \\ \mathrm{O} & 4.57629400 & 1.82519800 & -0.18498400 \\ \mathrm{O} & 4.65257900 & 0.74170500 & 2.10602600 \\ \mathrm{C} & 5.73590200 & -0.56744700 & 0.04367800 \\ \mathrm{C} & 6.17899000 & -0.47612700 & -1.27891800 \\ \mathrm{C} & 6.21424900 & -1.55327800 & 0.91246400 \\ \mathrm{C} & 7.11106600 & -1.40328100 & -1.73445500 \\ \mathrm{H} & 5.81523900 & 0.31432800 & -1.92626900 \\ \mathrm{C} & 7.14547700 & -2.46833200 & 0.43272100 \\ \mathrm{H} & 5.87634500 & -1.58875400 & 1.94246200 \\ \mathrm{C} & 7.61039500 & -2.40928800 & -0.89140100 \\ \mathrm{H} & 7.46776800 & -1.33867000 & -2.75867200 \\ \mathrm{H} & 7.52831400 & -3.23556200 & 1.09995200 \\ \mathrm{C} & 8.65179500 & -3.38133200 & -1.38469300 \\ \mathrm{H} & 9.66051100 & -2.99972700 & -1.17975100 \\ \mathrm{H} & 8.56445000 & -4.35155000 & -0.88628800 \\ \mathrm{H} & 8.57644800 & -3.53880700 & -2.46474500\end{array}$

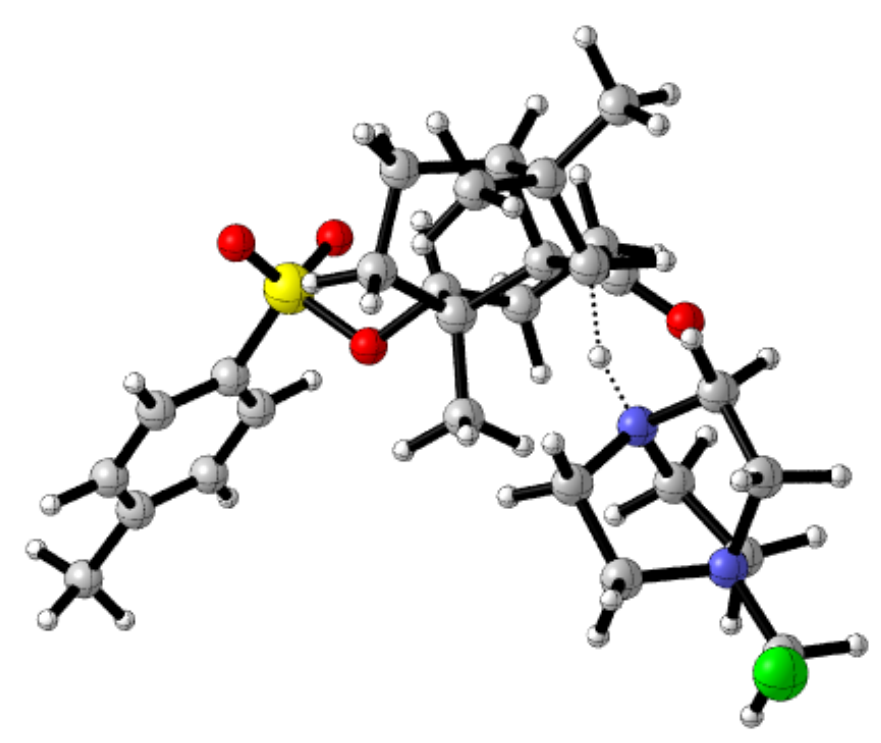

Figure S42. Transition state involving carbon C14 (bottom hydrogen) using SRD.

Zero-point correction=

Thermal correction to Energy=

Thermal correction to Enthalpy=

Thermal correction to Gibbs Free Energy=
0.672281 (Hartree/Particle)

0.707800

0.708744

0.601539 
Sum of electronic and zero-point Energies=

Sum of electronic and thermal Energies=

Sum of electronic and thermal Enthalpies=

Sum of electronic and thermal Free Energies=
$-2359.645795$

$-2359.610276$

$-2359.609332$

$-2359.716537$

Number of Imaginary Frequencies $=1$

E (Single Point Energy) $\left[\operatorname{IEFPCM}_{(\mathrm{CH} 3 \mathrm{CN})} \mathrm{U \omega B} 97 \mathrm{X}-\mathrm{D} / 6-311++\mathrm{G}(2 \mathrm{~d}, 2 \mathrm{p})\right]=-2360.61793073$

22

$\mathrm{C}$

C

C

$\mathrm{H}$

$\mathrm{H}$

C

$\mathrm{H}$

$\mathrm{H}$

$\mathrm{H}$

$\mathrm{H}$

C

$\mathrm{H}$

$\mathrm{H}$

$\mathrm{H}$

$\mathrm{H}$

$\mathrm{H}$

$\mathrm{H}$

$\mathrm{N}$

$\mathrm{N}$

C

$\mathrm{H}$

$\mathrm{H}$

$\mathrm{O}$

$\mathrm{Cl}$

C

C

C

C

C

C

$\mathrm{H}$

C

C

$\mathrm{H}$

$\mathrm{H}$

C

$\begin{array}{rrr}3.64437300 & 1.07893600 & -1.29072800 \\ 3.21597800 & 0.92188800 & 1.11553900 \\ 4.84470100 & -0.56627800 & 0.05795500 \\ 4.58205400 & 2.24941100 & -0.90787000 \\ 2.64598000 & 1.44707200 & -1.53476300 \\ 4.02128800 & 0.51353200 & -2.14578300 \\ 4.45185900 & 1.75597000 & 1.51787100 \\ 2.91408900 & 0.21225900 & 1.89239800 \\ 2.35566400 & 1.55333300 & 0.89026100 \\ 4.78362700 & -1.13324800 & 0.98894700 \\ 4.97455700 & -1.27119300 & -0.76611300 \\ 5.99301800 & 0.46907900 & 0.08300500 \\ 4.03086600 & 3.15353800 & -0.63941800 \\ 5.28113900 & 2.48979000 & -1.70986200 \\ 5.01942500 & 1.30099000 & 2.33276700 \\ 4.17216900 & 2.77046700 & 1.81043400 \\ 6.70219800 & 0.26796900 & 0.88878600 \\ 6.54260100 & 0.51152800 & -0.85743500 \\ 3.54407100 & 0.15012000 & -0.12602100 \\ 5.39637600 & 1.85180400 & 0.31918500 \\ 6.47086000 & 2.88964500 & 0.63763700 \\ 6.93297800 & 2.59364400 & 1.58031400 \\ 5.96725300 & 3.85149500 & 0.74205700 \\ 1.37544500 & -1.33560400 & 2.55420600 \\ 7.70924100 & 3.00643200 & -0.61589200 \\ 0.41832000 & -1.82217300 & 1.96821200 \\ -0.86180600 & -2.17326000 & 2.67263800 \\ 0.41924300 & -1.87226300 & 0.42306000 \\ -1.87955200 & -1.09242000 & 2.24441900 \\ -0.69327600 & -0.85387400 & -0.09371300 \\ -2.01444400 & -1.09738300 & 0.72361800 \\ -1.22240100 & -3.16262900 & 2.36494600 \\ 0.22947800 & -3.17375100 & -0.51025800 \\ -0.87598800 & -2.83444400 & -1.52269800 \\ -0.69405800 & -2.17381300 & 3.75175600 \\ -2.85944100 & -1.30761300 & 2.67877800 \\ 1.72469000 & -3.09806200 & -1.04727900\end{array}$




$\begin{array}{lrrr}\mathrm{C} & 1.86895500 & -1.96391700 & -0.03056200 \\ \mathrm{H} & 2.67822900 & -0.70825200 & -0.27609100 \\ \mathrm{H} & 2.52278700 & -2.21101100 & 0.81440800 \\ \mathrm{C} & 2.57275000 & -4.32088900 & -0.66641000 \\ \mathrm{H} & 2.45286100 & -4.59242800 & 0.38807300 \\ \mathrm{H} & 2.28161000 & -5.18880900 & -1.26889000 \\ \mathrm{H} & 3.63870200 & -4.13986500 & -0.85337000 \\ \mathrm{C} & 1.92347300 & -2.78758700 & -2.53492400 \\ \mathrm{H} & 1.58433500 & -3.62252500 & -3.15572300 \\ \mathrm{H} & 1.40422000 & -1.88731200 & -2.86984000 \\ \mathrm{H} & 2.99416700 & -2.65921400 & -2.74056000 \\ \mathrm{H} & -1.56540400 & -0.10726800 & 2.60790000 \\ \mathrm{C} & -0.96281400 & -1.30158800 & -1.54822200 \\ \mathrm{H} & -0.20864200 & -0.86881300 & -2.21540800 \\ \mathrm{C} & -0.25864800 & 0.61148000 & 0.01249800 \\ \mathrm{H} & -1.07145800 & 1.26573700 & -0.31149900 \\ \mathrm{H} & 0.00866400 & 0.89665600 & 1.03682100 \\ \mathrm{H} & 0.60131100 & 0.79665600 & -0.64527300 \\ \mathrm{H} & -2.40344200 & -2.08032900 & 0.44000600 \\ \mathrm{H} & -1.93941200 & -0.95313500 & -1.89352500 \\ \mathrm{H} & -0.67046800 & -3.26296300 & -2.50717300 \\ \mathrm{H} & -1.82559600 & -3.26845400 & -1.19368900 \\ \mathrm{H} & 0.07704800 & -4.09649600 & 0.05732500 \\ \mathrm{O} & -2.96118600 & -0.08387800 & 0.32292700 \\ \mathrm{~S} & -4.47224300 & -0.69879400 & -0.11888700 \\ \mathrm{O} & -4.30394000 & -1.27228600 & -1.45504900 \\ \mathrm{O} & -4.91471900 & -1.53926800 & 0.99192600 \\ \mathrm{C} & -5.39287300 & 0.80799800 & -0.19129300 \\ \mathrm{C} & -5.52531100 & 1.46863200 & -1.41558800 \\ \mathrm{C} & -6.01160000 & 1.28208800 & 0.96970900 \\ \mathrm{C} & -6.28417600 & 2.63439600 & -1.46496400 \\ \mathrm{H} & -5.06164800 & 1.06534000 & -2.30924100 \\ \mathrm{C} & -6.76388400 & 2.44951100 & 0.89408300 \\ \mathrm{H} & -5.92002000 & 0.73748300 & 1.90322600 \\ \mathrm{C} & -6.91591500 & 3.14178800 & -0.31867300 \\ \mathrm{H} & -6.40081100 & 3.15236400 & -2.41290900 \\ \mathrm{H} & -7.25412700 & 2.82392900 & 1.78849900 \\ & -7.77255600 & 4.38034600 & -0.39374700 \\ \mathrm{H} & -7.82288800 & 4.11065100 & -0.56390500 \\ \mathrm{H} & -7.46618900 & 5.03333600 & -1.21628700\end{array}$




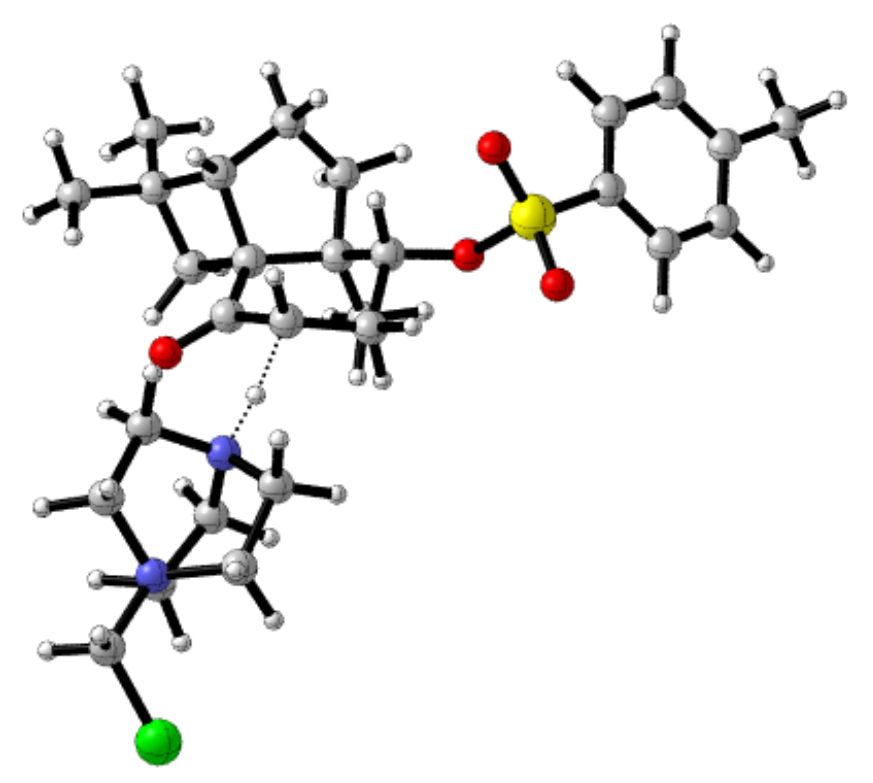

Figure S43. Transition state involving carbon C3 (top hydrogen) using SRD.

Zero-point correction $=$

Thermal correction to Energy=

Thermal correction to Enthalpy=

Thermal correction to Gibbs Free Energy=

Sum of electronic and zero-point Energies=

Sum of electronic and thermal Energies=

Sum of electronic and thermal Enthalpies=

Sum of electronic and thermal Free Energies=
0.671481 (Hartree/Particle)

0.707252

0.708197

0.599666

$-2359.661901$

$-2359.626130$

$-2359.625186$

$-2359.733717$

Number of Imaginary Frequencies $=1$

E (Single Point Energy) $\left[\right.$ IEFPCM $\left._{(\mathrm{CH} 3 \mathrm{CN})} \mathrm{U} \omega \mathrm{B} 97 \mathrm{X}-\mathrm{D} / 6-311++\mathrm{G}(2 \mathrm{~d}, 2 \mathrm{p})\right]=-2360.62349078$

22

$\mathrm{C}$

C

$3.64543500 \quad-1.98755600-0.85991100$

$\begin{array}{lll}4.32169700 & 0.33626800 & -1.22538800\end{array}$

$\begin{array}{llll}\mathrm{C} & 3.89196900 & -0.46913700 & 1.04450600\end{array}$

$\begin{array}{llll}\mathrm{C} & 5.08246500 & -2.46476200 & -0.53321500\end{array}$

$\mathrm{H} \quad 3.44119800 \quad-2.01367500-1.93259700$

$\mathrm{H} \quad 2.90484000 \quad-2.61235500-0.35565700$

C $\quad 5.77274400 \quad-0.20109400-1.25411400$

$\mathrm{H} \quad 4.23654800 \quad 1.33176100 \quad-0.78892500$

$\begin{array}{llll}\mathrm{H} & 3.90219100 & 0.36626600 & -2.23348300\end{array}$

$\begin{array}{llll}\mathrm{H} & 3.59146900 & 0.51874000 & 1.39569500\end{array}$

$\mathrm{H} \quad 3.34569900 \quad-1.23008500 \quad 1.60629200$ 


\begin{tabular}{|c|c|c|c|}
\hline $\mathrm{C}$ & 5.42176900 & -0.66730600 & 1.14731100 \\
\hline $\mathrm{H}$ & 5.55303700 & -2.95323500 & -1.38913400 \\
\hline $\mathrm{H}$ & 5.11267200 & -3.14953400 & 0.31422900 \\
\hline $\mathrm{H}$ & 6.49739900 & 0.59359300 & -1.06365800 \\
\hline $\mathrm{H}$ & 6.02261100 & -0.68314800 & -2.20191000 \\
\hline $\mathrm{H}$ & 5.95233000 & 0.27594900 & 1.29632600 \\
\hline $\mathrm{H}$ & 5.69290400 & -1.35342400 & 1.95049700 \\
\hline $\mathrm{N}$ & 3.48541900 & -0.57985800 & -0.39115300 \\
\hline $\mathrm{N}$ & 5.93583000 & -1.25580600 & -0.16254400 \\
\hline $\mathrm{C}$ & 7.41744700 & -1.61655900 & -0.06902100 \\
\hline $\mathrm{H}$ & 7.94826300 & -0.71420500 & 0.23684000 \\
\hline $\mathrm{H}$ & 7.73519500 & -1.93096600 & -1.06394600 \\
\hline $\mathrm{O}$ & 2.23716400 & 2.06509200 & 0.29342200 \\
\hline $\mathrm{Cl}$ & 7.74143900 & -2.90984500 & 1.08909800 \\
\hline $\mathrm{C}$ & 1.11213200 & 1.63490700 & 0.04539400 \\
\hline $\mathrm{C}$ & 0.93452800 & 0.32586200 & -0.68407100 \\
\hline $\mathrm{C}$ & -0.16558100 & 2.37787300 & 0.38648400 \\
\hline $\mathrm{C}$ & -0.11569100 & -0.58210900 & -0.16270300 \\
\hline $\mathrm{C}$ & -1.40014000 & 1.48921800 & 0.76203200 \\
\hline $\mathrm{C}$ & -1.47087900 & 0.25348600 & -0.18115900 \\
\hline $\mathrm{H}$ & 0.93154000 & 0.45027500 & -1.77525800 \\
\hline $\mathrm{C}$ & -0.65617200 & 3.38310000 & -0.77089200 \\
\hline $\mathrm{C}$ & -2.17167300 & 3.14086800 & -0.89739700 \\
\hline $\mathrm{H}$ & 2.31351300 & -0.23578500 & -0.50119600 \\
\hline $\mathrm{C}$ & -0.16160100 & 4.59989700 & 0.09239600 \\
\hline $\mathrm{C}$ & 0.10563300 & 3.63336500 & 1.28506000 \\
\hline $\mathrm{H}$ & 1.11039500 & 3.67079400 & 1.71096000 \\
\hline $\mathrm{C}$ & 1.12526900 & 5.20799900 & -0.48101000 \\
\hline $\mathrm{H}$ & 1.88835200 & 4.44677800 & -0.67485900 \\
\hline $\mathrm{H}$ & 0.91820800 & 5.73185800 & -1.42179500 \\
\hline $\mathrm{H}$ & 1.55207400 & 5.93530900 & 0.21912400 \\
\hline $\mathrm{C}$ & -1.17441300 & 5.71153000 & 0.37570500 \\
\hline $\mathrm{H}$ & -1.43337600 & 6.25676200 & -0.53937700 \\
\hline $\mathrm{H}$ & -2.10233800 & 5.33855500 & 0.82102700 \\
\hline $\mathrm{H}$ & -0.74455700 & 6.43534200 & 1.07773900 \\
\hline $\mathrm{H}$ & -0.26221900 & -1.47207300 & -0.77947600 \\
\hline $\mathrm{C}$ & -2.59766200 & 2.41319600 & 0.38956700 \\
\hline $\mathrm{H}$ & -3.52233600 & 1.83982000 & 0.27689300 \\
\hline $\mathrm{C}$ & -1.42761600 & 1.05647600 & 2.23639300 \\
\hline $\mathrm{H}$ & -2.32515600 & 0.46947300 & 2.44588600 \\
\hline $\mathrm{H}$ & -0.55886400 & 0.44750900 & 2.51513700 \\
\hline $\mathrm{H}$ & -1.43760500 & 1.92648400 & 2.89738100 \\
\hline $\mathrm{H}$ & -1.64297400 & 0.56662900 & -1.21436400 \\
\hline $\mathrm{H}$ & -2.72030400 & 4.07523300 & -1.04668700 \\
\hline $\mathrm{H}$ & -0.14676700 & 3.30227000 & -1.73727600 \\
\hline $\mathrm{H}$ & -0.62320900 & 3.72819400 & 2.0933350 \\
\hline
\end{tabular}




$\begin{array}{lrrr}\mathrm{H} & -2.37927500 & 2.52125300 & -1.77708600 \\ \mathrm{H} & -2.76375000 & 3.12536900 & 1.20505700 \\ \mathrm{H} & 0.05067200 & -0.89051500 & 0.87453400 \\ \mathrm{O} & -2.52675900 & -0.59492200 & 0.22591200 \\ \mathrm{~S} & -3.17163700 & -1.62158400 & -0.98074400 \\ \mathrm{O} & -3.18915200 & -0.83646800 & -2.21356700 \\ \mathrm{O} & -2.38835000 & -2.85214100 & -0.88894900 \\ \mathrm{C} & -4.79429200 & -1.84614300 & -0.33168400 \\ \mathrm{C} & -5.82805700 & -1.01984500 & -0.78842000 \\ \mathrm{C} & -5.01928700 & -2.85965600 & 0.60696000 \\ \mathrm{C} & -7.10728500 & -1.21625600 & -0.28111600 \\ \mathrm{H} & -5.63405600 & -0.25690900 & -1.53448600 \\ \mathrm{C} & -6.30764000 & -3.03309100 & 1.09923200 \\ \mathrm{H} & -4.20733700 & -3.50210400 & 0.92970000 \\ \mathrm{C} & -7.37050100 & -2.22110900 & 0.66593000 \\ \mathrm{H} & -7.91984400 & -0.58609700 & -0.63129300 \\ \mathrm{H} & -6.49725500 & -3.81767700 & 1.82618200 \\ \mathrm{C} & -8.76857600 & -2.44640500 & 1.17898900 \\ \mathrm{H} & -8.76419700 & -2.85782800 & 2.19263600 \\ \mathrm{H} & -9.35048300 & -1.52004400 & 1.18216700 \\ \mathrm{H} & -9.29957700 & -3.16279000 & 0.53823200\end{array}$

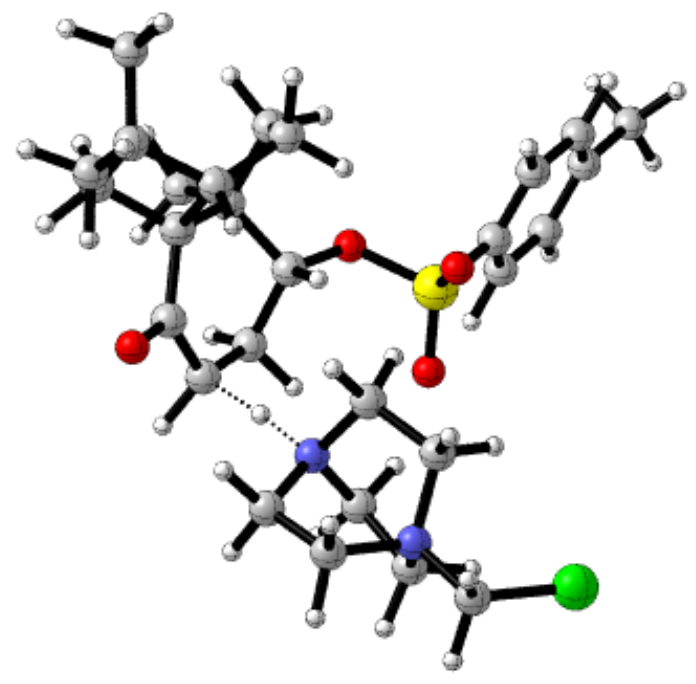

Figure S44. Transition state involving carbon C3 (bottom hydrogen) using SRD. 
Zero-point correction=

Thermal correction to Energy=

Thermal correction to Enthalpy=

Thermal correction to Gibbs Free Energy=

Sum of electronic and zero-point Energies $=$

Sum of electronic and thermal Energies=

Sum of electronic and thermal Enthalpies=

Sum of electronic and thermal Free Energies=
0.671319 (Hartree/Particle)

0.706898

0.707843

0.599905

$-2359.667608$

$-2359.632029$

$-2359.631085$

$-2359.739022$

Number of Imaginary Frequencies $=1$

E (Single Point Energy) IEFPCM $_{(\mathrm{CH} 3 \mathrm{CN})} \mathrm{U \omega B}$ B97X-D/6-311++G(2d,2p)] = -2360.62293097

$\begin{array}{lrrr}2 \mathrm{2} & & & \\ \mathrm{C} & 2.16748000 & -0.88686600 & -0.64911800 \\ \mathrm{C} & 3.98431500 & -0.60462000 & 0.96352900 \\ \mathrm{C} & 2.06009800 & -2.01877700 & 1.51387700 \\ \mathrm{C} & 2.72205300 & -2.22245800 & -1.20246300 \\ \mathrm{H} & 2.60369300 & -0.02370900 & -1.15688100 \\ \mathrm{H} & 1.08046600 & -0.86148200 & -0.77050400 \\ \mathrm{C} & 4.71313600 & -1.71941900 & 0.17354000 \\ \mathrm{H} & 4.21463700 & -0.65014300 & 2.03015500 \\ \mathrm{H} & 4.25525600 & 0.38884000 & 0.60043000 \\ \mathrm{H} & 2.16202500 & -1.85706300 & 2.58958400 \\ \mathrm{H} & 1.00118800 & -2.17624600 & 1.28732500 \\ \mathrm{C} & 2.94405800 & -3.20663800 & 1.06208900 \\ \mathrm{H} & 3.25351300 & -2.08544000 & -2.14664000 \\ \mathrm{H} & 1.93503000 & -2.96213100 & -1.34952300 \\ \mathrm{H} & 5.50641400 & -2.18073400 & 0.76581400 \\ \mathrm{H} & 5.14239800 & -1.35245000 & -0.76128200 \\ \mathrm{H} & 3.69280400 & -3.48196400 & 1.80863900 \\ \mathrm{H} & 2.34601300 & -4.08653900 & 0.82242900 \\ \mathrm{~N} & 2.51127500 & -0.78401200 & 0.80218800 \\ \mathrm{~N} & 3.70785800 & -2.80680200 & -0.19580200 \\ \mathrm{C} & 4.48192100 & -3.98681800 & -0.77737200 \\ \mathrm{H} & 5.12709500 & -4.37002900 & 0.01413600 \\ \mathrm{H} & 5.07758500 & -3.60304400 & -1.60651700 \\ \mathrm{O} & 2.87215200 & 2.70979800 & 0.97438100 \\ \mathrm{Cl} & 3.43291900 & -5.28362300 & -1.35817500 \\ \mathrm{C} & 1.69433700 & 2.42506500 & 1.11809900 \\ \mathrm{C} & 1.31053200 & 1.30187500 & 2.05000000 \\ \mathrm{C} & 0.57002100 & 3.20525800 & 0.39405000 \\ \mathrm{C} & -0.12193700 & 0.90005200 & 2.17476300 \\ \mathrm{C} & -0.90964100 & 2.71381800 & 0.58804200 \\ \mathrm{C} & -0.79464500 & 1.18918100 & 0.83717600 \\ \mathrm{H} & 1.90529300 & 1.31804600 & 2.97006900 \\ \mathrm{C} & 0.83766300 & 3.24677900 & -1.19138900\end{array}$




\begin{tabular}{|c|c|c|c|}
\hline $\mathrm{C}$ & -0.46075800 & 2.77141100 & -1.85231800 \\
\hline $\mathrm{H}$ & 1.93872000 & 0.23903300 & 1.32488200 \\
\hline $\mathrm{C}$ & 1.14952600 & 4.78806000 & -1.07375400 \\
\hline $\mathrm{C}$ & 0.83710300 & 4.74615900 & 0.45901700 \\
\hline $\mathrm{H}$ & 1.67611600 & 5.03238700 & 1.09694400 \\
\hline $\mathrm{C}$ & 2.62243400 & 5.09947800 & -1.37544200 \\
\hline $\mathrm{H}$ & 3.29872600 & 4.46363500 & -0.79755600 \\
\hline $\mathrm{H}$ & 2.83390800 & 4.95569800 & -2.44191200 \\
\hline $\mathrm{H}$ & 2.84775400 & 6.14344200 & -1.13029300 \\
\hline $\mathrm{C}$ & 0.24936300 & 5.73290300 & -1.87648700 \\
\hline $\mathrm{H}$ & 0.42062400 & 5.61711000 & -2.95289800 \\
\hline $\mathrm{H}$ & -0.81751600 & 5.58595000 & -1.68577900 \\
\hline $\mathrm{H}$ & 0.48692300 & 6.77029600 & -1.61525200 \\
\hline $\mathrm{H}$ & -0.20480000 & -0.16838600 & 2.41606900 \\
\hline $\mathrm{C}$ & -1.56291500 & 2.97180900 & -0.79780900 \\
\hline $\mathrm{H}$ & -2.42834100 & 2.32318000 & -0.95502100 \\
\hline $\mathrm{C}$ & -1.69198600 & 3.41298700 & 1.71478100 \\
\hline $\mathrm{H}$ & -2.64010500 & 2.89529700 & 1.89370300 \\
\hline $\mathrm{H}$ & -1.14369600 & 3.45663800 & 2.66147800 \\
\hline $\mathrm{H}$ & -1.93570700 & 4.44176400 & 1.43901200 \\
\hline $\mathrm{H}$ & -0.16640700 & 0.79072000 & 0.03235900 \\
\hline $\mathrm{H}$ & -0.67245500 & 3.31559100 & -2.77807800 \\
\hline $\mathrm{H}$ & 1.71326000 & 2.67912000 & -1.52196000 \\
\hline $\mathrm{H}$ & -0.04258500 & 5.31692200 & 0.76299000 \\
\hline $\mathrm{H}$ & -0.38099200 & 1.71199700 & -2.12456300 \\
\hline $\mathrm{H}$ & -1.93278700 & 4.00096400 & -0.82211000 \\
\hline $\mathrm{H}$ & -0.62199100 & 1.41052600 & 3.00887700 \\
\hline $\mathrm{O}$ & -2.07241600 & 0.51905600 & 0.73050400 \\
\hline $\mathrm{S}$ & -1.93551800 & -1.03810900 & 0.15519000 \\
\hline $\mathrm{O}$ & -1.22846600 & -0.96592000 & -1.13668600 \\
\hline $\mathrm{O}$ & -1.28601000 & -1.84617800 & 1.20603600 \\
\hline $\mathrm{C}$ & -3.62836400 & -1.46180000 & -0.03953000 \\
\hline $\mathrm{C}$ & -4.24791800 & -1.23190500 & -1.27337900 \\
\hline $\mathrm{C}$ & -4.31775300 & -2.01835900 & 1.04406200 \\
\hline $\mathrm{C}$ & -5.59037700 & -1.56680000 & -1.41078600 \\
\hline $\mathrm{H}$ & -3.68879500 & -0.81836800 & -2.10562700 \\
\hline $\mathrm{C}$ & -5.65954600 & -2.34185800 & 0.87724200 \\
\hline $\mathrm{H}$ & -3.81325600 & -2.20486100 & 1.98582000 \\
\hline $\mathrm{C}$ & -6.31906500 & -2.11911700 & -0.34362600 \\
\hline $\mathrm{H}$ & -6.08198600 & -1.40269700 & -2.36531900 \\
\hline $\mathrm{H}$ & -6.20539700 & -2.78084000 & 1.70734800 \\
\hline $\mathrm{C}$ & -7.78056800 & -2.44963000 & -0.49927100 \\
\hline $\mathrm{H}$ & -8.07287200 & -3.28946600 & 0.13772000 \\
\hline $\mathrm{H}$ & -8.39937200 & -1.58991000 & -0.21063000 \\
\hline $\mathrm{H}$ & -8.02910500 & -2.69728200 & -1.53522600 \\
\hline
\end{tabular}




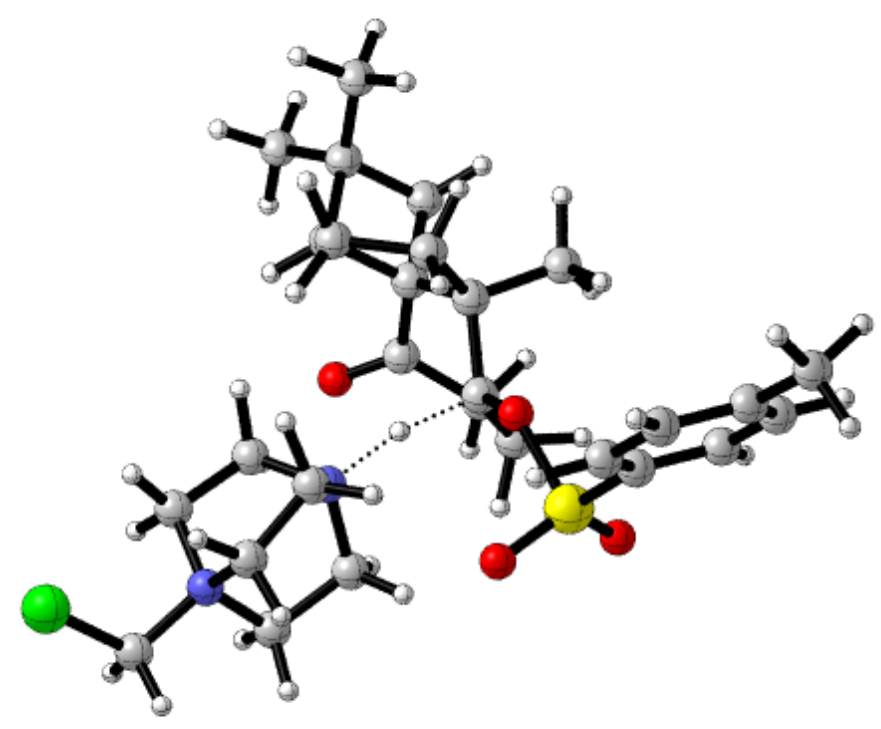

Figure S45. Transition state involving carbon C7 using SRD.

Zero-point correction=

Thermal correction to Energy=

Thermal correction to Enthalpy=

Thermal correction to Gibbs Free Energy=

Sum of electronic and zero-point Energies=

Sum of electronic and thermal Energies=

Sum of electronic and thermal Enthalpies $=$

Sum of electronic and thermal Free Energies=
0.671952 (Hartree/Particle)

0.707641

0.708585

0.602642

$-2359.662229$

$-2359.626540$

$-2359.625596$

$-2359.731539$

Number of Imaginary Frequencies $=1$

E (Single Point Energy) [IEFPCM $(\mathrm{CH} 3 \mathrm{CN}) \mathrm{U \omega B}$ 97X-D/6-311++G(2d,2p)] = -2360.61371366

22

$\begin{array}{lrrr}\mathrm{C} & 2.96677800 & -0.42738700 & 0.11455300 \\ \mathrm{C} & 1.56766600 & -1.74850100 & 1.59657200 \\ \mathrm{C} & 1.14614900 & -1.75517300 & -0.81068000 \\ \mathrm{C} & 3.87656800 & -1.62078500 & -0.27481900 \\ \mathrm{H} & 3.30793700 & 0.08366100 & 1.01464700 \\ \mathrm{H} & 2.94467800 & 0.30677800 & -0.68971600 \\ \mathrm{C} & 2.68471400 & -2.82086100 & 1.51699300 \\ \mathrm{H} & 0.58055400 & -2.20389900 & 1.69131300 \\ \mathrm{H} & 1.74893400 & -1.09098200 & 2.44834500 \\ \mathrm{H} & 0.08155200 & -1.96607200 & -0.71354200 \\ \mathrm{H} & 1.31903600 & -1.17134100 & -1.71644700 \\ \mathrm{C} & 1.94833400 & -3.07771000 & -0.82119500 \\ \mathrm{H} & 4.82332600 & -1.59960900 & 0.26952200\end{array}$




\begin{tabular}{|c|c|c|c|}
\hline $\mathrm{H}$ & 4.09275900 & -1.65464400 & -1.34270000 \\
\hline $\mathrm{H}$ & 2.31655200 & -3.80328000 & 1.82088100 \\
\hline $\mathrm{H}$ & 3.55312000 & -2.56454400 & 2.12771700 \\
\hline $\mathrm{H}$ & 1.36838000 & -3.91331400 & -0.42205100 \\
\hline $\mathrm{H}$ & 2.29896100 & -3.33521200 & -1.82130700 \\
\hline $\mathrm{N}$ & 1.58016500 & -0.92603400 & 0.35230600 \\
\hline $\mathrm{N}$ & 3.16897400 & -2.92371500 & 0.07663800 \\
\hline $\mathrm{C}$ & 4.09882400 & -4.12665100 & -0.02718700 \\
\hline $\mathrm{H}$ & 3.50596200 & -5.01358300 & 0.19941200 \\
\hline $\mathrm{H}$ & 4.88665000 & -3.99073400 & 0.71466100 \\
\hline $\mathrm{O}$ & 2.73627900 & 2.12328600 & 1.68224000 \\
\hline $\mathrm{Cl}$ & 4.82589000 & -4.30247300 & -1.62869400 \\
\hline $\mathrm{C}$ & 1.55108800 & 2.40335000 & 1.57585900 \\
\hline $\mathrm{C}$ & 0.56975100 & 2.17681300 & 2.70990600 \\
\hline $\mathrm{C}$ & 0.93351800 & 2.92790000 & 0.29714900 \\
\hline $\mathrm{C}$ & -0.42795000 & 1.05735700 & 2.31050700 \\
\hline $\mathrm{C}$ & -0.43758800 & 2.21344500 & -0.02673500 \\
\hline $\mathrm{C}$ & -0.52189000 & 0.88884900 & 0.78596300 \\
\hline $\mathrm{H}$ & 1.11081400 & 1.91717000 & 3.62204900 \\
\hline $\mathrm{C}$ & 1.81814800 & 2.87724900 & -0.98897600 \\
\hline $\mathrm{C}$ & 1.04998800 & 2.05883700 & -2.03840400 \\
\hline $\mathrm{H}$ & 0.02217300 & 3.10730800 & 2.89666200 \\
\hline $\mathrm{H}$ & -0.16192000 & 0.11456400 & 2.79459400 \\
\hline $\mathrm{C}$ & 1.84750000 & 4.45172500 & -1.05640000 \\
\hline $\mathrm{C}$ & 0.97299700 & 4.50480500 & 0.23701500 \\
\hline $\mathrm{H}$ & 1.47352700 & 4.96123100 & 1.09692900 \\
\hline $\mathrm{C}$ & 3.25975700 & 5.00777200 & -0.84373200 \\
\hline $\mathrm{H}$ & 3.74212300 & 4.55451100 & 0.03007300 \\
\hline $\mathrm{H}$ & 3.89188500 & 4.81370500 & -1.71860100 \\
\hline $\mathrm{H}$ & 3.23157000 & 6.09216300 & -0.68794500 \\
\hline $\mathrm{C}$ & 1.19983600 & 5.10467800 & -2.27874500 \\
\hline $\mathrm{H}$ & 1.78369200 & 4.91594300 & -3.18715500 \\
\hline $\mathrm{H}$ & 0.17569300 & 4.75761800 & -2.45781700 \\
\hline $\mathrm{H}$ & 1.15408900 & 6.19092900 & -2.14166400 \\
\hline $\mathrm{H}$ & -1.43287400 & 1.28201400 & 2.68362900 \\
\hline $\mathrm{C}$ & -0.41840000 & 2.01383000 & -1.57141200 \\
\hline $\mathrm{H}$ & -0.94241500 & 1.10152800 & -1.87156900 \\
\hline $\mathrm{C}$ & -1.71611100 & 3.00957600 & 0.37475300 \\
\hline $\mathrm{H}$ & -2.60857100 & 2.41953100 & 0.14749600 \\
\hline $\mathrm{H}$ & -1.73861400 & 3.28278800 & 1.43311500 \\
\hline $\mathrm{H}$ & -1.77340500 & 3.93108700 & -0.20793700 \\
\hline $\mathrm{H}$ & 0.61450300 & 0.10866400 & 0.51273000 \\
\hline $\mathrm{H}$ & 1.13981100 & 2.47192800 & -3.04745300 \\
\hline $\mathrm{H}$ & 2.82304200 & 2.48536000 & -0.80844500 \\
\hline $\mathrm{H}$ & -0.00040200 & 4.98584700 & 0.12277300 \\
\hline $\mathrm{H}$ & 1.46308300 & 1.04057900 & -2.09901300 \\
\hline
\end{tabular}




$\begin{array}{lrrr}\mathrm{H} & -0.97232700 & 2.84080500 & -2.02538800 \\ \mathrm{O} & -1.52481100 & 0.09101100 & 0.27356400 \\ \mathrm{~S} & -2.36212400 & -1.23172500 & 1.08676600 \\ \mathrm{O} & -1.52593200 & -2.38279900 & 0.72192200 \\ \mathrm{O} & -2.54897500 & -0.86552000 & 2.48086500 \\ \mathrm{C} & -3.86608400 & -1.19815800 & 0.18955200 \\ \mathrm{C} & -3.95913900 & -1.86601200 & -1.03912000 \\ \mathrm{C} & -4.95685000 & -0.51643100 & 0.74871400 \\ \mathrm{C} & -5.17327100 & -1.84181200 & -1.71283400 \\ \mathrm{H} & -3.11116300 & -2.40579000 & -1.44573400 \\ \mathrm{C} & -6.15818400 & -0.51076400 & 0.05092300 \\ \mathrm{H} & -4.87027700 & -0.02365900 & 1.71089000 \\ \mathrm{C} & -6.29082100 & -1.16949100 & -1.18432500 \\ \mathrm{H} & -5.26312500 & -2.36101900 & -2.66249300 \\ \mathrm{H} & -7.01341300 & 0.00626100 & 0.47600500 \\ \mathrm{C} & -7.60996900 & -1.18425200 & -1.90835800 \\ \mathrm{H} & -8.21962700 & -2.03108000 & -1.56627000 \\ \mathrm{H} & -8.18408300 & -0.27317700 & -1.71684300 \\ \mathrm{H} & -7.47694400 & -1.29318000 & -2.98837800\end{array}$

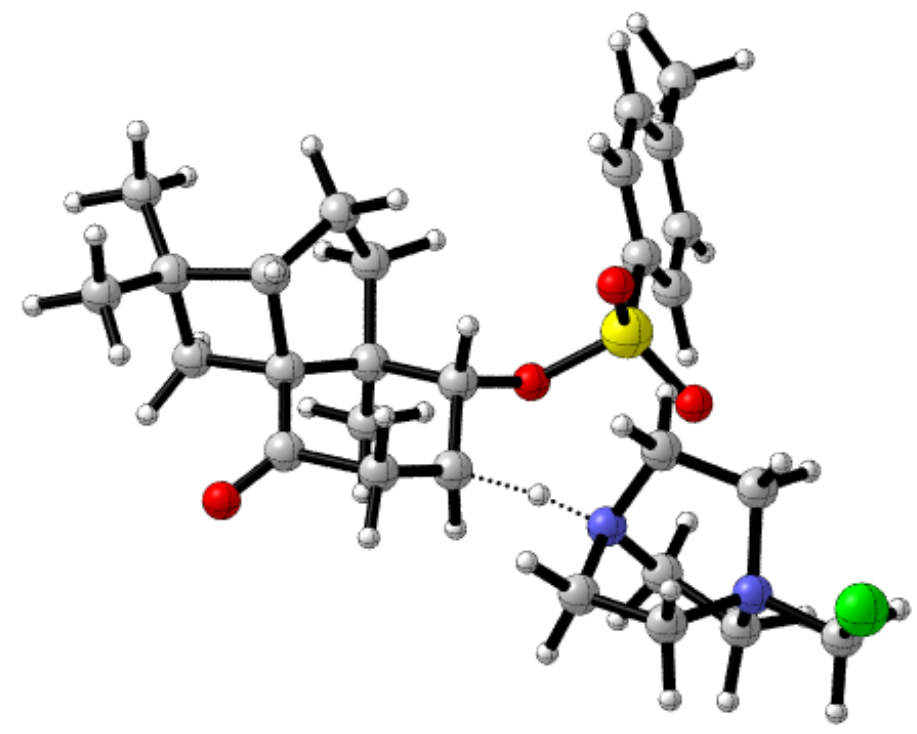

Figure S46. Transition state involving carbon C7 (bottom hydrogen) using SRD.

Zero-point correction=

Thermal correction to Energy=

Thermal correction to Enthalpy=

Thermal correction to Gibbs Free Energy=

Sum of electronic and zero-point Energies=
0.672069 (Hartree/Particle)

0.707682

0.708626

0.601547

$-2359.674619$ 
Sum of electronic and thermal Energies=

Sum of electronic and thermal Enthalpies=

Sum of electronic and thermal Free Energies=
$-2359.639006$

$-2359.638061$

$-2359.745141$

Number of Imaginary Frequencies $=1$

E (Single Point Energy) IEFPCM $_{(\mathrm{CH} 3 \mathrm{CN})} \mathrm{U \omega B}$ B7X-D/6-311++G(2d,2p)] = -2360.63094606

$\begin{array}{lrrr}2 \mathrm{C} & & & \\ \mathrm{C} & -3.54270600 & -2.06134200 & 0.41525700 \\ \mathrm{C} & -3.11685800 & 0.08626800 & 1.49200500 \\ \mathrm{C} & -2.96589200 & -0.13505700 & -0.95869800 \\ \mathrm{C} & -5.00975400 & -1.71676300 & 0.05424500 \\ \mathrm{H} & -3.45881800 & -2.47871400 & 1.42116500 \\ \mathrm{H} & -3.13445000 & -2.78484500 & -0.29358100 \\ \mathrm{C} & -4.65340200 & 0.25984100 & 1.49044400 \\ \mathrm{H} & -2.58350600 & 1.03113900 & 1.36241600 \\ \mathrm{H} & -2.78499700 & -0.37759000 & 2.42364500 \\ \mathrm{H} & -2.20731600 & 0.63430100 & -1.11399800 \\ \mathrm{H} & -2.84917400 & -0.88992600 & -1.73970300 \\ \mathrm{C} & -4.38404300 & 0.46791500 & -0.95268000 \\ \mathrm{H} & -5.71722000 & -2.18493700 & 0.74198100 \\ \mathrm{H} & -5.27007500 & -2.01197900 & -0.96207100 \\ \mathrm{H} & -4.93405900 & 1.30713900 & 1.62239900 \\ \mathrm{H} & -5.14878900 & -0.33647600 & 2.26020000 \\ \mathrm{H} & -4.37571400 & 1.53630100 & -0.72427000 \\ \mathrm{H} & -4.89841800 & 0.31064100 & -1.90150000 \\ \mathrm{~N} & -2.72676300 & -0.81210200 & 0.35808000 \\ \mathrm{~N} & -5.20209500 & -0.20684500 & 0.14556400 \\ \mathrm{C} & -6.67390700 & 0.18517400 & 0.06899100 \\ \mathrm{H} & -6.71695100 & 1.27474000 & 0.09058600 \\ \mathrm{H} & -7.16721400 & -0.23657100 & 0.94557600 \\ \mathrm{O} & 1.57058700 & -4.52216400 & 1.09953200 \\ \mathrm{Cl} & -7.46995200 & -0.40443200 & -1.39428300 \\ \mathrm{C} & 1.51001200 & -3.40874600 & 0.65406300 \\ \mathrm{C} & 0.05897200 & -2.84766800 & 0.28566000 \\ \mathrm{C} & 2.66942300 & -2.48096600 & 0.36993200 \\ \mathrm{C} & -0.08932000 & -1.48359800 & 0.84846600 \\ \mathrm{C} & 2.42467700 & -0.99859300 & 0.78332800 \\ \mathrm{C} & 0.96486900 & -0.53624800 & 0.35046500 \\ \mathrm{H} & 0.03717000 & -2.85583600 & -0.81319600 \\ \mathrm{C} & 3.17647100 & -2.47442000 & -1.14493300 \\ \mathrm{C} & 3.39974400 & -0.98584600 & -1.48166300 \\ \mathrm{H} & -0.64768100 & -3.58401200 & 0.67273300 \\ \mathrm{H} & -1.53155800 & -1.07971600 & 0.48606800 \\ \mathrm{C} & 4.41858800 & -3.34036200 & -0.71563100 \\ \mathrm{C} & 4.03724000 & -3.12022700 & 0.77999100\end{array}$




$\begin{array}{lrrr}\mathrm{H} & 3.94291900 & -4.02059800 & 1.39144100 \\ \mathrm{C} & 4.25632600 & -4.80133000 & -1.15731900 \\ \mathrm{H} & 3.28250300 & -5.21525900 & -0.87477000 \\ \mathrm{H} & 4.36036900 & -4.89004600 & -2.24520700 \\ \mathrm{H} & 5.02610900 & -5.42930900 & -0.69531000 \\ \mathrm{C} & 5.80120800 & -2.82511700 & -1.11745900 \\ \mathrm{H} & 5.94061300 & -2.86243300 & -2.20424900 \\ \mathrm{H} & 5.98472400 & -1.79707100 & -0.78668000 \\ \mathrm{H} & 6.57705100 & -3.45418700 & -0.66643300 \\ \mathrm{H} & -0.24538100 & -1.44198400 & 1.93300000 \\ \mathrm{C} & 3.42579800 & -0.24077600 & -0.13381400 \\ \mathrm{H} & 3.18074300 & 0.82235000 & -0.21820100 \\ \mathrm{C} & 2.64311200 & -0.74155800 & 2.28250600 \\ \mathrm{H} & 2.51222900 & 0.31653900 & 2.52057100 \\ \mathrm{H} & 1.95503700 & -1.31942100 & 2.91029300 \\ \mathrm{H} & 3.65798600 & -1.02333700 & 2.57317800 \\ \mathrm{H} & 4.31577600 & -0.82861300 & -2.05794300 \\ \mathrm{H} & 2.53764800 & -2.96993200 & -1.88455600 \\ \mathrm{H} & 4.69549100 & -2.41559500 & 1.29343700 \\ \mathrm{H} & 2.57977000 & -0.61868600 & -2.11095700 \\ \mathrm{H} & 4.42107400 & -0.29469600 & 0.32083900 \\ \mathrm{O} & 0.76421300 & 0.80014400 & 0.88256300 \\ \mathrm{~S} & 0.03562700 & 1.96033400 & -0.04988200 \\ \mathrm{O} & -0.16363900 & 1.39342900 & -1.39330800 \\ \mathrm{O} & -1.15997600 & 2.35458800 & 0.71219600 \\ \mathrm{C} & 1.19857000 & 3.28275100 & -0.07887500 \\ \mathrm{C} & 2.00646400 & 3.44912200 & -1.20924000 \\ \mathrm{C} & 1.29042000 & 4.13615300 & 1.02735300 \\ \mathrm{C} & 2.92384300 & 4.49573300 & -1.21961400 \\ \mathrm{H} & 1.90439300 & 2.79016800 & -2.06459300 \\ \mathrm{C} & 2.21846000 & 5.17020900 & 0.99001900 \\ \mathrm{H} & 0.64512400 & 4.00135100 & 1.88867600 \\ \mathrm{C} & 3.04747700 & 5.37038400 & -0.12789900 \\ \mathrm{H} & 3.55054700 & 4.64098900 & -2.09478300 \\ \mathrm{H} & 2.29887000 & 5.83995000 & 1.84146200 \\ \mathrm{C} & 4.02313800 & 6.51769300 & -0.16375500 \\ \mathrm{H} & 4.84980600 & 6.32281400 & -0.85254800 \\ \mathrm{H} & 3.52289900 & 7.43454500 & -0.50177000 \\ \mathrm{H} & 4.43847600 & 6.72192200 & 0.82772000 \\ & 0.93209600 & -0.48422600 & -0.74036900\end{array}$




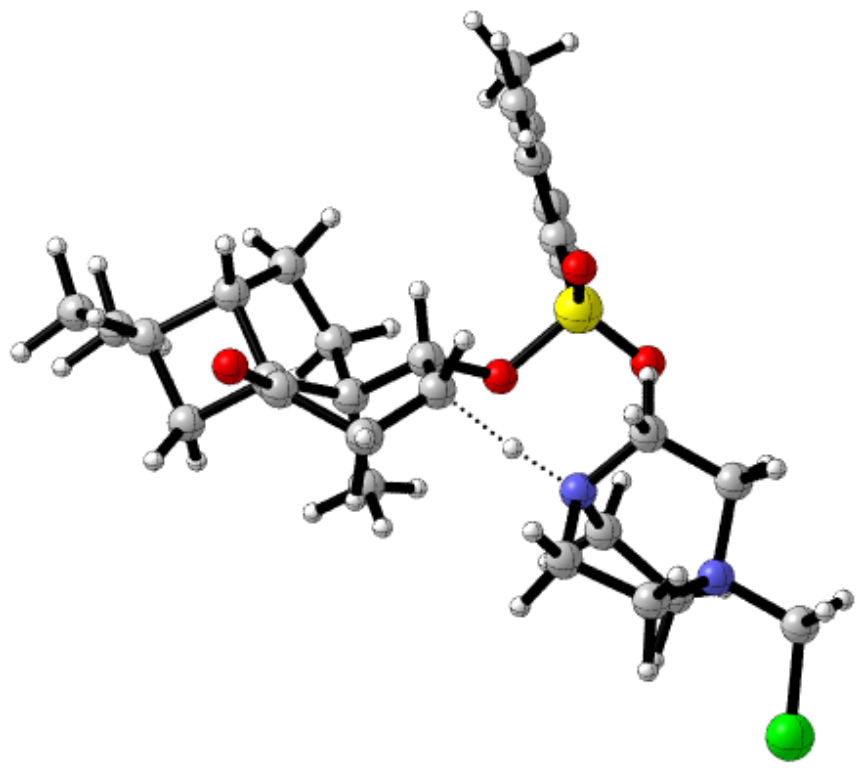

Figure S47. Transition state involving carbon C7 (top hydrogen) using SRD.

Zero-point correction $=$

Thermal correction to Energy=

Thermal correction to Enthalpy=

Thermal correction to Gibbs Free Energy=

Sum of electronic and zero-point Energies=

Sum of electronic and thermal Energies $=$

Sum of electronic and thermal Enthalpies=

Sum of electronic and thermal Free Energies=
0.672176 (Hartree/Particle)

0.707686

0.708630

0.602658

$-2359.667311$

$-2359.631800$

$-2359.630856$

$-2359.736828$

Number of Imaginary Frequencies $=1$

E (Single Point Energy) IEFPCM $\left._{(\mathrm{CH} 3 \mathrm{CN})} \mathrm{U} \omega \mathrm{B} 97 \mathrm{X}-\mathrm{D} / 6-311++\mathrm{G}(2 \mathrm{~d}, 2 \mathrm{p})\right]=-2360.62620885$

22

$\begin{array}{lrrr}\mathrm{C} & -2.88053000 & -0.03408000 & 0.88891300 \\ \mathrm{C} & -3.14515000 & 0.29810600 & -1.53373000 \\ \mathrm{C} & -3.58249400 & -1.88139700 & -0.54538100 \\ \mathrm{C} & -4.38737200 & 0.12439600 & 1.19764100 \\ \mathrm{H} & -2.35979300 & 0.92506700 & 0.87451100 \\ \mathrm{H} & -2.39924000 & -0.68045600 & 1.62542300 \\ \mathrm{C} & -4.50552400 & 0.90627400 & -1.13883700 \\ \mathrm{H} & -3.20387100 & -0.25840400 & -2.47192300 \\ \mathrm{H} & -2.38487700 & 1.07276400 & -1.64418200 \\ \mathrm{H} & -3.31941800 & -2.44360000 & -1.44445300 \\ \mathrm{H} & -3.37273600 & -2.50569400 & 0.32579600\end{array}$




\begin{tabular}{|c|c|c|c|}
\hline $\mathrm{C}$ & -5.06653800 & -1.44345900 & -0.59374400 \\
\hline $\mathrm{H}$ & -4.60601900 & 1.10729400 & 1.62078900 \\
\hline $\mathrm{H}$ & -4.77005800 & -0.63824300 & 1.87624100 \\
\hline $\mathrm{H}$ & -5.17385300 & 0.99216600 & -1.99851800 \\
\hline $\mathrm{H}$ & -4.39376700 & 1.88808100 & -0.67329900 \\
\hline $\mathrm{H}$ & -5.47465400 & -1.46356900 & -1.60684400 \\
\hline $\mathrm{H}$ & -5.69362900 & -2.06210200 & 0.04933900 \\
\hline $\mathrm{N}$ & -2.72451500 & -0.66245900 & -0.46277400 \\
\hline $\mathrm{N}$ & -5.16999200 & -0.00386200 & -0.10538200 \\
\hline $\mathrm{C}$ & -6.62158800 & 0.43304300 & 0.05231800 \\
\hline $\mathrm{H}$ & -7.11627000 & 0.26405100 & -0.90492300 \\
\hline $\mathrm{H}$ & -6.61167700 & 1.49577200 & 0.29740200 \\
\hline $\mathrm{O}$ & 1.93509400 & -3.94487300 & -1.76736000 \\
\hline $\mathrm{Cl}$ & -7.47698900 & -0.45526300 & 1.31840400 \\
\hline $\mathrm{C}$ & 1.55573900 & -3.14496900 & -0.95593100 \\
\hline $\mathrm{C}$ & 0.00192500 & -2.78393500 & -0.94446600 \\
\hline $\mathrm{C}$ & 2.39089800 & -2.45764100 & 0.09343900 \\
\hline $\mathrm{C}$ & -0.12009300 & -1.31065800 & -1.06682100 \\
\hline $\mathrm{C}$ & 1.69810300 & -1.23577500 & 0.79446200 \\
\hline $\mathrm{C}$ & 0.88170800 & -0.46350800 & -0.27798800 \\
\hline $\mathrm{H}$ & -0.47367300 & -3.33737200 & -1.75633600 \\
\hline $\mathrm{C}$ & 3.82836800 & -1.98639300 & -0.40305000 \\
\hline $\mathrm{C}$ & 3.97444100 & -0.54134900 & 0.09958300 \\
\hline $\mathrm{H}$ & -0.32538400 & -3.19464100 & 0.01905900 \\
\hline $\mathrm{H}$ & -1.53023000 & -0.97082100 & -0.65522500 \\
\hline $\mathrm{C}$ & 4.50618600 & -3.15043700 & 0.41091300 \\
\hline $\mathrm{C}$ & 3.11573100 & -3.51495500 & 1.01640700 \\
\hline $\mathrm{H}$ & 2.79227600 & -4.54886100 & 0.86591700 \\
\hline $\mathrm{C}$ & 5.06067100 & -4.24423300 & -0.51142200 \\
\hline $\mathrm{H}$ & 4.32764600 & -4.55895300 & -1.26042400 \\
\hline $\mathrm{H}$ & 5.95170200 & -3.88532200 & -1.03960300 \\
\hline $\mathrm{H}$ & 5.35168100 & -5.12623200 & 0.07053400 \\
\hline $\mathrm{C}$ & 5.56645100 & -2.75710200 & 1.44124900 \\
\hline $\mathrm{H}$ & 6.46853000 & -2.37158300 & 0.95233500 \\
\hline $\mathrm{H}$ & 5.21665700 & -1.99943800 & 2.15089500 \\
\hline $\mathrm{H}$ & 5.86280500 & -3.63696900 & 2.02376400 \\
\hline $\mathrm{H}$ & -0.22245300 & -0.95917800 & -2.09814100 \\
\hline $\mathrm{C}$ & 2.92300300 & -0.37057200 & 1.21153000 \\
\hline $\mathrm{H}$ & 2.64141500 & 0.67240500 & 1.38444300 \\
\hline $\mathrm{C}$ & 0.82563400 & -1.56441300 & 2.02088400 \\
\hline $\mathrm{H}$ & 0.52323300 & -0.64003900 & 2.52093200 \\
\hline $\mathrm{H}$ & -0.08528200 & -2.12306500 & 1.77387600 \\
\hline $\mathrm{H}$ & 1.38038900 & -2.16122800 & 2.74851700 \\
\hline $\mathrm{H}$ & 4.98564500 & -0.32641500 & 0.45649300 \\
\hline $\mathrm{H}$ & 3.99463700 & -2.07056500 & -1.48044900 \\
\hline $\mathrm{H}$ & 3.01772500 & -3.27523700 & 2.07816500 \\
\hline
\end{tabular}




$\begin{array}{lrrr}\mathrm{H} & 3.78520800 & 0.16028300 & -0.72203300 \\ \mathrm{H} & 3.30843800 & -0.75285400 & 2.16315200 \\ \mathrm{O} & 0.20571000 & 0.65373300 & 0.38931400 \\ \mathrm{~S} & -0.05740500 & 2.06016500 & -0.45999300 \\ \mathrm{O} & -0.20536100 & 1.68762100 & -1.87383700 \\ \mathrm{O} & -1.21353900 & 2.61547400 & 0.25528700 \\ \mathrm{C} & 1.37217500 & 3.06496000 & -0.23385400 \\ \mathrm{C} & 2.26627800 & 3.22357700 & -1.29913300 \\ \mathrm{C} & 1.57556600 & 3.69660400 & 1.00070000 \\ \mathrm{C} & 3.38555100 & 4.03013400 & -1.11163600 \\ \mathrm{H} & 2.07557700 & 2.74920000 & -2.25554900 \\ \mathrm{C} & 2.70260100 & 4.49334300 & 1.15940400 \\ \mathrm{H} & 0.86058900 & 3.58136200 & 1.80854600 \\ \mathrm{C} & 3.62367900 & 4.67687000 & 0.11148600 \\ \mathrm{H} & 4.08098200 & 4.16877800 & -1.93437500 \\ \mathrm{H} & 2.86858400 & 4.99272200 & 2.10972000 \\ \mathrm{C} & 4.81850100 & 5.57632200 & 0.29089500 \\ \mathrm{H} & 5.21647700 & 5.51681600 & 1.30851500 \\ \mathrm{H} & 5.61966800 & 5.32842800 & -0.41073700 \\ \mathrm{H} & 4.53790900 & 6.62264200 & 0.11319400 \\ \mathrm{H} & 1.57920700 & -0.03407200 & -1.00664300\end{array}$

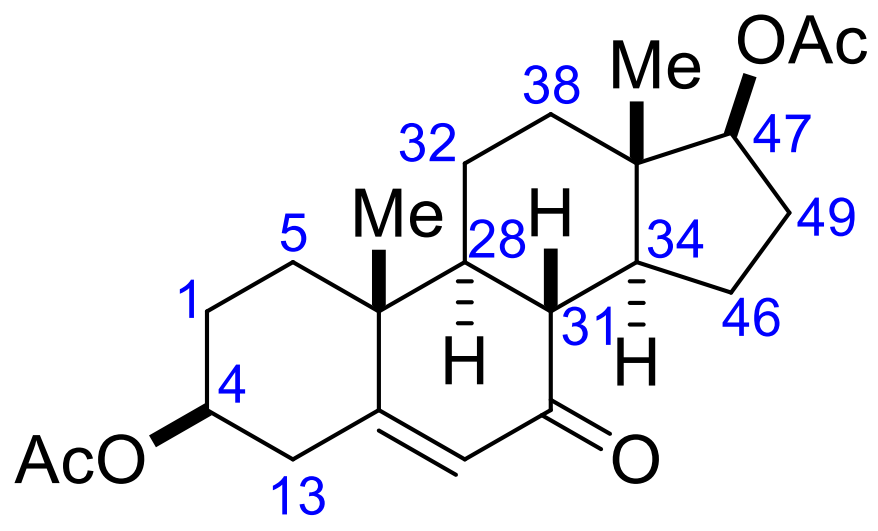

Figure S48. Depiction of 1 with Pertinent Carbon Atoms Labelled. Top Hydrogen is Denoted by (T), and the Bottom Hydrogen is Denoted by (B).

Table S5. Single point energies of all structures calculated at the $\operatorname{IEFPCM}_{(\mathrm{CH} 3 \mathrm{CN})} \mathrm{U \omega B} 97 \mathrm{X}-\mathrm{D} / 6$ $311++\mathrm{G}(2 \mathrm{~d}, 2 \mathrm{p})$ level of theory and thermal correction to Gibbs free energy and Gibbs free energy calculated at the UB3LYP/6-31G(d) level of theory. All energies are reported in Hartrees.

\begin{tabular}{|c|c|c|c|c|}
\hline Structure & $\begin{array}{c}\text { Single Point } \\
\text { Energies (E) } \\
\text { IEFPCM }_{(\mathrm{CH} 3 \mathrm{CN})} \mathrm{U} \\
\omega \mathrm{B} 97 \mathrm{X}-\mathrm{D} / 6- \\
311++\mathrm{G}(2 \mathrm{~d}, 2 \mathrm{p})\end{array}$ & $\begin{array}{c}\text { Thermal } \\
\text { Corrections } \\
\text { to Gibbs } \\
\text { Free }\end{array}$ & $\begin{array}{c}\text { Gibbs Free } \\
\text { Energies } \\
\text { (G) } \\
\text { UB3LYP/6- } \\
\text { 31G(d) }\end{array}$ & $\begin{array}{c}\text { Gibbs Free Energies } \\
(\mathrm{G}) \\
\text { IEFPCM }_{(\mathrm{CH} 3 \mathrm{CN})} \mathrm{U} \omega \mathrm{B} 9 \\
7 \mathrm{X}-\mathrm{D} / 6-\end{array}$ \\
\hline
\end{tabular}




\begin{tabular}{|c|c|c|c|c|}
\hline & & $\begin{array}{c}\text { Energies } \\
\text { (G) } \\
\text { UB3LYP/6- } \\
\mathbf{3 1 G ( d )}\end{array}$ & & $\begin{array}{c}\text { 311++G(2d,2p)//UB3 } \\
\text { LYP/6-31G(d) }\end{array}$ \\
\hline C31 SRD & -2116.37127013 & 0.663756 & -2115.423203 & -2115.707514 \\
\hline C34 SRD & -2116.36433475 & 0.663618 & -2115.422671 & -2115.700717 \\
\hline C46 (T) SRD & -2116.38173015 & 0.661494 & -2115.444766 & -2115.720236 \\
\hline C46 (B) SRD & -2116.38712802 & 0.662865 & -2115.456919 & -2115.724263 \\
\hline C49 (T) SRD & -2116.37835672 & 0.662659 & -2115.432861 & -2115.715698 \\
\hline C49 (B) SRD & -2116.38651245 & 0.662864 & -2115.450749 & -2115.723648 \\
\hline C47 SRD & -2116.38091986 & 0.663813 & -2115.438931 & -2115.717107 \\
\hline C38 (B) SRD & -2116.38545836 & 0.662688 & -2115.435494 & -2115.72277 \\
\hline C38 (T) SRD & -2116.38509348 & 0.663310 & -2115.439495 & -2115.721783 \\
\hline C32 (B) SRD & -2116.38215268 & 0.662589 & -2115.425484 & -2115.719564 \\
\hline C32 (T) SRD & -2116.36850650 & 0.662631 & -2115.410722 & -2115.705876 \\
\hline C28 SRD & -2116.37241681 & 0.661031 & -2115.410295 & -2115.711386 \\
\hline C5 (B) SRD & -2116.37265293 & 0.662609 & -2115.405493 & -2115.710044 \\
\hline C5 (T) SRD & -2116.37011876 & 0.659037 & -2115.393529 & -2115.711082 \\
\hline C1 (T) SRD & -2116.35370719 & 0.662907 & -2115.383868 & -2115.6908 \\
\hline C1 (B) SRD & -2116.37808574 & 0.662132 & -2115.414569 & -2115.715954 \\
\hline C4 & -2116.38032235 & 0.662372 & -2115.413498 & -2115.71795 \\
\hline C13 (B) SRD & -2116.37545493 & 0.663190 & -2115.426454 & -2115.712265 \\
\hline C13 (T) SRD & -2116.37872335 & 0.662222 & -2115.422453 & -2115.716501 \\
\hline
\end{tabular}

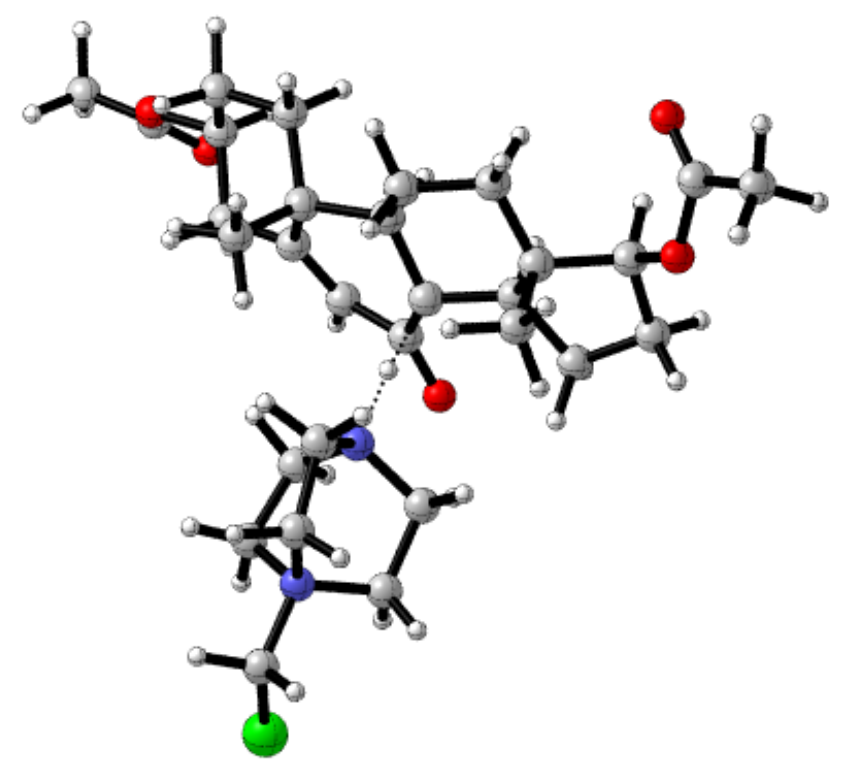

Figure S49. Transition state involving carbon C31 using SRD. 
Zero-point correction=

Thermal correction to Energy=

Thermal correction to Enthalpy=

Thermal correction to Gibbs Free Energy=

Sum of electronic and zero-point Energies $=$

Sum of electronic and thermal Energies=

Sum of electronic and thermal Enthalpies=

Sum of electronic and thermal Free Energies=
0.734834 (Hartree/Particle)

0.772436

0.773380

0.663756

$-2115.352125$

$-2115.314523$

$-2115.313579$

$-2115.423203$

Number of Imaginary Frequencies $=1$

E (Single Point Energy) $\left[\operatorname{IEFPCM}_{(\mathrm{CH} 3 \mathrm{CN})} \mathrm{U} \omega \mathrm{B} 97 \mathrm{X}-\mathrm{D} / 6-311++\mathrm{G}(2 \mathrm{~d}, 2 \mathrm{p})\right]=-2116.37127013$

22

$\mathrm{C}$

$\mathrm{C}$

2.24696500

2.20061500

0.68864400

1.52665900

$2.03659300-1.63518900$

C

$\mathrm{C}$

$-0.02558700$

$\begin{array}{ll}2.79949800 & 0.08493200\end{array}$

2.53806500

$3.71979100 \quad 0.61288200$

$\begin{array}{llll}\mathrm{H} & 3.10768000 & 1.60993700 & 0.37284300\end{array}$

$\begin{array}{llll}\mathrm{C} & 2.19556800 & 3.42307700 & -1.81050200\end{array}$

$\mathrm{H} \quad \begin{array}{llll}\mathrm{H} & 0.64529500 & 1.93305400 & -2.27046100\end{array}$

$\mathrm{H} \quad 2.23045000 \quad 1.24451100 \quad-1.89174800$

$\mathrm{H} \quad-0.92773200 \quad 2.44367200 \quad-0.41465100$

$\begin{array}{llll}\mathrm{H} & -0.19720800 & 2.77038400 & 1.16137000\end{array}$

$\begin{array}{llll}\mathrm{C} & 0.33836000 & 4.22124600 & -0.40288800\end{array}$

$\begin{array}{llll}\mathrm{H} & 3.60664100 & 3.92501200 & 0.51760700\end{array}$

$\begin{array}{llll}\mathrm{H} & 2.15872900 & 4.26084600 & 1.47984400\end{array}$

$\begin{array}{llll}\mathrm{H} & 1.84464800 & 3.92639900 & -2.71420800\end{array}$

$\mathrm{H} \quad 3.28517100 \quad 3.35711400 \quad-1.84779500$

$\mathrm{H} \quad-0.12643900 \quad 4.47141100 \quad-1.35964100$

$\begin{array}{llll}\mathrm{H} & 0.06275700 & 4.98058500 & 0.33005200\end{array}$

$\mathrm{N} \quad 1.10304000 \quad 1.86251000 \quad-0.21560400$

$\begin{array}{llll}\mathrm{N} & 1.84349200 & 4.29793700 & -0.61449800\end{array}$

$\begin{array}{llll}\mathrm{C} & 2.29891600 & 5.72404400 & -0.91259400\end{array}$

$\begin{array}{llll}\mathrm{H} & 1.72408000 & 6.07255400 & -1.77161900\end{array}$

$\begin{array}{llll}\mathrm{H} & 3.36154900 & 5.67788700 & -1.15434600\end{array}$

$\begin{array}{llll}\mathrm{O} & 0.02394100 & 0.70373400 & 2.45242400\end{array}$

$\begin{array}{llll}\mathrm{Cl} & 2.05105300 & 6.82470600 & 0.44583900\end{array}$

$\begin{array}{llll}\mathrm{C} & -0.56006100 & 0.09680600 & 1.55660500\end{array}$

$\begin{array}{llll}\mathrm{C} & -2.00786800 & 0.16077500 & 1.38832500\end{array}$

$\begin{array}{llll}\mathrm{C} & 0.21831700 & -0.72226600 & 0.52482100\end{array}$

$\begin{array}{llll}\mathrm{C} & -2.68224300 & -0.25602800 & 0.28730600\end{array}$

$\begin{array}{llll}\mathrm{C} & -2.01745800 & -1.00317700 & -0.86247700\end{array}$

$\begin{array}{llll}\text { C } & -4.15381700 & 0.04854500 & 0.17534200\end{array}$

$\begin{array}{llll}\mathrm{C} & -2.94148300 & -2.21286000 & -1.24819500\end{array}$

$\begin{array}{llll}\text { C } & -4.97963800 & -1.20787300 & -0.18345100\end{array}$ 


\begin{tabular}{|c|c|c|c|}
\hline $\mathrm{H}$ & -4.31919600 & 0.78104300 & -0.62826500 \\
\hline $\mathrm{H}$ & -4.52950200 & 0.48276000 & 1.10401600 \\
\hline $\mathrm{C}$ & -4.42323200 & -1.86505400 & -1.43870900 \\
\hline $\mathrm{H}$ & -2.55669400 & -2.67543400 & -2.16241500 \\
\hline $\mathrm{H}$ & -2.86224500 & -2.97112200 & -0.45817500 \\
\hline $\mathrm{H}$ & -4.95651500 & -1.89847200 & 0.66643200 \\
\hline $\mathrm{H}$ & -4.98242200 & -2.78129100 & -1.65479200 \\
\hline $\mathrm{H}$ & -4.57502200 & -1.19833800 & -2.29635100 \\
\hline $\mathrm{H}$ & -2.52924900 & 0.64750800 & 2.20908900 \\
\hline C & -6.34029400 & -0.84490600 & -0.44143000 \\
\hline C & -7.13071400 & -0.63450400 & 0.66269100 \\
\hline $\mathrm{O}$ & -6.68608300 & -0.65217400 & 1.78721700 \\
\hline $\mathrm{C}$ & -8.56274700 & -0.40508100 & 0.26501200 \\
\hline $\mathrm{H}$ & -8.62840100 & 0.33982800 & -0.53344500 \\
\hline $\mathrm{H}$ & -8.98938900 & -1.33718200 & -0.12209100 \\
\hline $\mathrm{H}$ & -9.13348000 & -0.08112600 & 1.13546500 \\
\hline $\mathrm{C}$ & 1.37099200 & -3.05020000 & -0.98798100 \\
\hline $\mathrm{H}$ & 0.95244000 & -3.91818800 & -0.46524000 \\
\hline $\mathrm{H}$ & 1.96390800 & -3.45739700 & -1.81361600 \\
\hline $\mathrm{C}$ & 2.26757700 & -2.25586900 & -0.02424700 \\
\hline $\mathrm{C}$ & 1.37937400 & -1.54757600 & 1.06239800 \\
\hline $\mathrm{C}$ & 3.16980100 & -1.28687600 & -0.81084300 \\
\hline $\mathrm{H}$ & 3.72605900 & -0.60092500 & -0.16213600 \\
\hline $\mathrm{H}$ & 3.91356700 & -1.85616100 & -1.37461300 \\
\hline $\mathrm{H}$ & 2.59307800 & -0.70523600 & -1.53858100 \\
\hline $\mathrm{C}$ & 3.13838100 & -3.11421300 & 0.94095800 \\
\hline $\mathrm{C}$ & 2.37173200 & -1.08061000 & 2.14716600 \\
\hline $\mathrm{C}$ & 3.46305000 & -2.19002100 & 2.13756900 \\
\hline $\mathrm{H}$ & 2.54779100 & -3.98085900 & 1.25276700 \\
\hline $\mathrm{H}$ & 4.46264700 & -1.76640700 & 2.00515300 \\
\hline $\mathrm{H}$ & 3.48095900 & -2.75493500 & 3.07284300 \\
\hline $\mathrm{O}$ & 4.36297300 & -3.59196500 & 0.37604300 \\
\hline $\mathrm{C}$ & 4.33181000 & -4.83413600 & -0.21407400 \\
\hline $\mathrm{O}$ & 3.30596400 & -5.45306700 & -0.37321000 \\
\hline $\mathrm{C}$ & 5.71643800 & -5.28495100 & -0.59114700 \\
\hline $\mathrm{H}$ & 5.65012600 & -6.16238800 & -1.23476500 \\
\hline $\mathrm{H}$ & 6.26718900 & -4.48366300 & -1.09201100 \\
\hline $\mathrm{H}$ & 6.27247500 & -5.54656000 & 0.31637600 \\
\hline $\mathrm{C}$ & -1.89057400 & -0.05537300 & -2.08208000 \\
\hline $\mathrm{H}$ & -2.85413400 & 0.37035200 & -2.37062300 \\
\hline $\mathrm{H}$ & -1.21321100 & 0.78198500 & -1.87027100 \\
\hline $\mathrm{H}$ & -1.49798600 & -0.59433000 & -2.94896000 \\
\hline $\mathrm{H}$ & 1.88453600 & -0.97408600 & 3.11897500 \\
\hline $\mathrm{H}$ & 0.69666900 & 0.58330500 & 0.01632300 \\
\hline $\mathrm{H}$ & -0.90451500 & -2.43923500 & 0.22669200 \\
\hline $\mathrm{C}$ & -0.63905000 & -1.57914000 & -0.4118520 \\
\hline
\end{tabular}




$\begin{array}{lrrr}\mathrm{C} & 0.22313200 & -2.19384100 & -1.54856900 \\ \mathrm{H} & 0.61127600 & -1.39918500 & -2.19886100 \\ \mathrm{H} & -0.40931900 & -2.82157400 & -2.18231000 \\ \mathrm{H} & 2.81851900 & -0.11090900 & 1.91688800 \\ \mathrm{H} & 1.96855500 & 1.90748200 & 1.70088900 \\ \mathrm{H} & 0.84622800 & -2.39680400 & 1.53235900\end{array}$

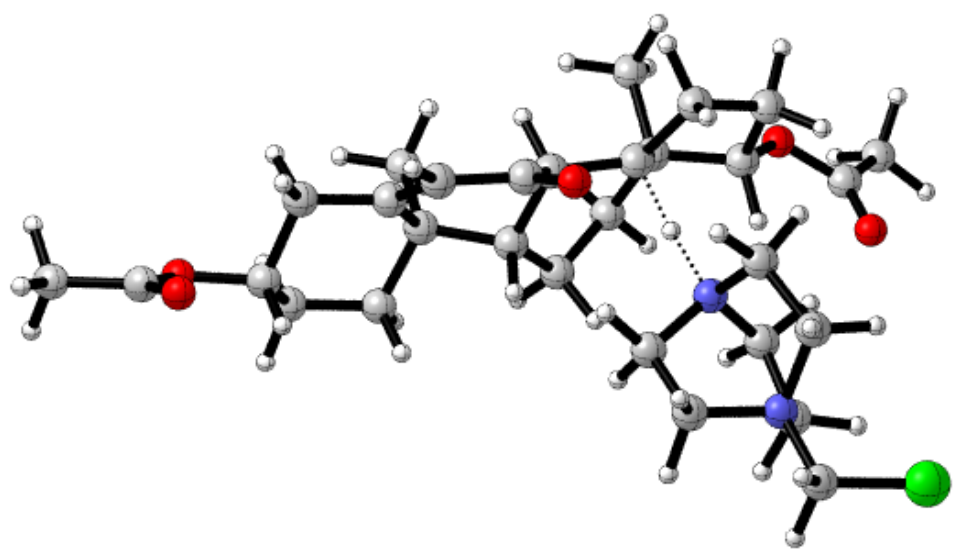

Figure S50. Transition state involving carbon C34 using SRD.

Zero-point correction $=$

Thermal correction to Energy=

Thermal correction to Enthalpy=

Thermal correction to Gibbs Free Energy=

Sum of electronic and zero-point Energies=

Sum of electronic and thermal Energies $=$

Sum of electronic and thermal Enthalpies=

Sum of electronic and thermal Free Energies=
0.735107 (Hartree/Particle)

0.772613

0.773557

0.663618

$-2115.351183$

$-2115.313677$

$-2115.312732$

$-2115.422671$

Number of Imaginary Frequencies $=1$

E (Single Point Energy) $\left[\operatorname{IEFPCM}_{(\mathrm{CH} 3 \mathrm{CN})} \mathrm{U \omega B}\right.$ B97X-D/6-311++G(2d,2p)] = -2116.36433475

22

$\begin{array}{lrrr}\mathrm{C} & -2.66932600 & 1.45315800 & 1.42260300 \\ \mathrm{C} & -0.98280900 & 1.99325800 & -0.23198200 \\ \mathrm{C} & -2.99605700 & 0.79237700 & -0.90477400 \\ \mathrm{C} & -3.55108400 & 2.68479800 & 1.09120800 \\ \mathrm{H} & -1.88887100 & 1.68008400 & 2.15103200 \\ \mathrm{C} & -1.65626500 & 3.38747900 & -0.31327900 \\ \mathrm{H} & -0.57608200 & 1.69307800 & -1.19912200\end{array}$




\begin{tabular}{|c|c|c|c|}
\hline $\mathrm{H}$ & -0.18644200 & 2.00271000 & 0.51270300 \\
\hline $\mathrm{H}$ & -2.51280200 & 0.30226700 & -1.75248300 \\
\hline $\mathrm{H}$ & -3.79438100 & 0.13690600 & -0.54967200 \\
\hline $\mathrm{C}$ & -3.54784400 & 2.17653900 & -1.33382200 \\
\hline $\mathrm{H}$ & -3.40238100 & 3.49326700 & 1.81043700 \\
\hline $\mathrm{H}$ & -4.61304200 & 2.44142000 & 1.05668100 \\
\hline $\mathrm{H}$ & -1.39423000 & 3.90882600 & -1.23663500 \\
\hline $\mathrm{H}$ & -1.39164500 & 4.02161400 & 0.53573800 \\
\hline $\mathrm{H}$ & -3.12516900 & 2.52070000 & -2.28064000 \\
\hline $\mathrm{H}$ & -4.63520100 & 2.16706600 & -1.41792100 \\
\hline $\mathrm{N}$ & -1.99071200 & 0.97561600 & 0.18267400 \\
\hline $\mathrm{N}$ & -3.16866400 & 3.21275700 & -0.28476300 \\
\hline $\mathrm{C}$ & -3.81209400 & 4.56571800 & -0.57465200 \\
\hline $\mathrm{H}$ & -3.51814400 & 4.85199200 & -1.58519600 \\
\hline $\mathrm{H}$ & -3.41632800 & 5.27258500 & 0.15564300 \\
\hline $\mathrm{O}$ & 0.51622000 & 0.88936700 & 2.36168800 \\
\hline $\mathrm{Cl}$ & -5.57473800 & 4.53067700 & -0.46433400 \\
\hline $\mathrm{C}$ & 1.27451900 & 0.13049300 & 1.75231600 \\
\hline $\mathrm{C}$ & 2.72339200 & 0.29465900 & 1.65833900 \\
\hline $\mathrm{C}$ & 0.77174000 & -1.05254500 & 0.94623100 \\
\hline $\mathrm{C}$ & 3.46531900 & -0.29371700 & 0.68009700 \\
\hline $\mathrm{C}$ & 2.85037900 & -1.05329900 & -0.51181100 \\
\hline $\mathrm{C}$ & 4.96196600 & -0.13217100 & 0.67796000 \\
\hline $\mathrm{C}$ & 3.45259300 & -0.44715200 & -1.82139300 \\
\hline $\mathrm{C}$ & 5.46803700 & 0.47969400 & -0.64545000 \\
\hline $\mathrm{H}$ & 5.43697400 & -1.11727700 & 0.79083800 \\
\hline $\mathrm{H}$ & 5.28299500 & 0.48793400 & 1.51769600 \\
\hline $\mathrm{C}$ & 4.98395300 & -0.33664800 & -1.83669800 \\
\hline $\mathrm{H}$ & 3.12483400 & -1.04974400 & -2.67603000 \\
\hline $\mathrm{H}$ & 3.02989800 & 0.55784500 & -1.96538000 \\
\hline $\mathrm{H}$ & 5.11336000 & 1.51400900 & -0.71180000 \\
\hline $\mathrm{H}$ & 5.30569600 & 0.14108200 & -2.76818800 \\
\hline $\mathrm{H}$ & 5.45482700 & -1.32632300 & -1.81588300 \\
\hline $\mathrm{H}$ & 3.19420200 & 0.93175100 & 2.40272100 \\
\hline $\mathrm{O}$ & 6.90061200 & 0.49034100 & -0.66735600 \\
\hline $\mathrm{C}$ & 7.50648700 & 1.53368800 & -0.01452400 \\
\hline $\mathrm{O}$ & 6.87840800 & 2.37639800 & 0.58520700 \\
\hline $\mathrm{C}$ & 9.00203700 & 1.46395700 & -0.16133800 \\
\hline $\mathrm{H}$ & 9.45951200 & 2.28325400 & 0.39327500 \\
\hline $\mathrm{H}$ & 9.37487600 & 0.50481900 & 0.21216400 \\
\hline $\mathrm{H}$ & 9.27878900 & 1.53064900 & -1.21872800 \\
\hline $\mathrm{C}$ & -0.43226800 & -2.66735300 & -1.23685800 \\
\hline $\mathrm{H}$ & -1.21738100 & -2.79954700 & -1.99115000 \\
\hline $\mathrm{H}$ & 0.19524000 & -3.55924000 & -1.31990500 \\
\hline $\mathrm{C}$ & -1.06283300 & -2.64158500 & 0.18395500 \\
\hline $\mathrm{C}$ & -0.68162700 & -1.41282100 & 1.01054700 \\
\hline
\end{tabular}




$\begin{array}{lrrr}\mathrm{C} & -0.60444700 & -3.95117300 & 0.91256000 \\ \mathrm{H} & -0.98764700 & -4.04339400 & 1.93050400 \\ \mathrm{H} & -0.97911600 & -4.80208000 & 0.33736000 \\ \mathrm{H} & 0.48667500 & -4.01836700 & 0.94451400 \\ \mathrm{C} & -2.59846700 & -2.51118700 & 0.29856300 \\ \mathrm{C} & -1.53784100 & -1.49019800 & 2.27548100 \\ \mathrm{C} & -2.84795100 & -2.17555500 & 1.79325600 \\ \mathrm{H} & -2.92421100 & -1.68335100 & -0.33068000 \\ \mathrm{H} & -3.04513100 & -3.09702900 & 2.34694100 \\ \mathrm{H} & -3.73743900 & -1.54882900 & 1.91601700 \\ \mathrm{O} & -3.31104900 & -3.65747200 & -0.15078500 \\ \mathrm{C} & -4.53956600 & -3.39688400 & -0.70758500 \\ \mathrm{O} & -4.96777600 & -2.26661500 & -0.82811500 \\ \mathrm{C} & -5.23602300 & -4.66278300 & -1.11227800 \\ \mathrm{H} & -5.48499900 & -5.24680100 & -0.21917100 \\ \mathrm{H} & -6.15009100 & -4.42050800 & -1.65425800 \\ \mathrm{H} & -4.57907900 & -5.28131100 & -1.73113800 \\ \mathrm{C} & 3.19581900 & -2.56036500 & -0.43439800 \\ \mathrm{H} & 4.27478200 & -2.72601300 & -0.46834700 \\ \mathrm{H} & 2.83517600 & -3.03011500 & 0.48723500 \\ \mathrm{H} & 2.75997800 & -3.09365700 & -1.28624700 \\ \mathrm{H} & -0.99750100 & -2.11898100 & 2.99977800 \\ \mathrm{H} & -1.31247100 & -0.26110900 & 0.49528500 \\ \mathrm{H} & 1.28647000 & -1.94761100 & 1.33777500 \\ \mathrm{H} & 1.23415400 & 0.28368100 & -0.65803500 \\ \mathrm{C} & 1.30865700 & -0.80113600 & -0.49992200 \\ \mathrm{C} & 0.41152700 & -1.42921900 & -1.60598600 \\ \mathrm{H} & 1.01195300 & -1.66643300 & -2.48947600 \\ \mathrm{H} & -0.27633700 & -0.63910900 & -1.94367700 \\ \mathrm{H} & -3.29530200 & 0.65041000 & 1.81176500 \\ \mathrm{H} & -1.66364400 & -0.53754900 & 2.78867100\end{array}$




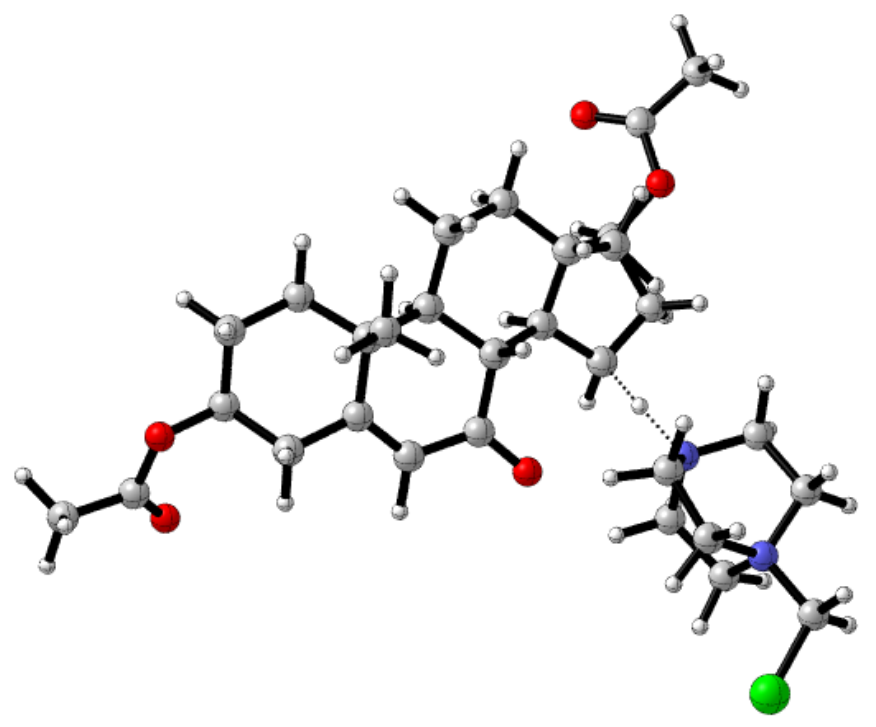

Figure S51. Transition state involving carbon C46 (top hydrogen) using SRD.

Zero-point correction $=$

Thermal correction to Energy=

Thermal correction to Enthalpy=

Thermal correction to Gibbs Free Energy=

Sum of electronic and zero-point Energies=

Sum of electronic and thermal Energies=

Sum of electronic and thermal Enthalpies=

Sum of electronic and thermal Free Energies=
0.734294 (Hartree/Particle)

0.771889

0.772833

0.661494

$-2115.371967$

$-2115.334372$

$-2115.333428$

$-2115.444766$

Number of Imaginary Frequencies $=1$

E (Single Point Energy) [IEFPCM $($ IH3CN $) U \omega B$ B7X-D/6-311++G(2d,2p)] = -2116.38173015

\section{2}

$\mathrm{C}$

$\mathrm{C}$

$\begin{array}{llll}3.16288700 & -1.81544300 & -1.46525300\end{array}$

C

$\begin{array}{llll}4.57127100 & -0.35505400 & -0.10823400\end{array}$

C $\quad 4.24818800 \quad-2.90042100 \quad-1.25705700$

$\mathrm{H} \quad 3.35744600 \quad-1.25424400 \quad-2.38196000$

$\mathrm{H} \quad 2.15435900 \quad-2.22582300 \quad-1.51891000$

C $\quad \begin{array}{llll}5.47348400 & -1.51855900 & 0.38601900\end{array}$

$\mathrm{H} \quad 4.54751700 \quad 0.44727600 \quad 0.63219200$

$\mathrm{H} \quad 4.93095800 \quad 0.05747200 \quad-1.05313300$

$\mathrm{H} \quad 2.84310400 \quad-0.86848100 \quad 1.75842800$

$\mathrm{H} \quad \begin{array}{llll}\mathrm{H} & 1.65619200 & -1.76600800 & 0.77664900\end{array}$

C $\quad 3.52819900 \quad-2.85983600 \quad 1.11587700$

$\mathrm{H} \quad 5.12453600 \quad-2.74822500 \quad-1.89165400$ 


\begin{tabular}{|c|c|c|c|}
\hline $\mathrm{H}$ & 3.85838900 & -3.90364900 & -1.43278100 \\
\hline $\mathrm{H}$ & 5.70323500 & -1.43978900 & 1.45079100 \\
\hline $\mathrm{H}$ & 6.41085500 & -1.56760800 & -0.17229000 \\
\hline $\mathrm{H}$ & 3.89987200 & -2.96166400 & 2.13786300 \\
\hline $\mathrm{H}$ & 2.95074600 & -3.74977000 & 0.86582900 \\
\hline $\mathrm{N}$ & 3.19392800 & -0.86888900 & -0.31283500 \\
\hline $\mathrm{N}$ & 4.73793800 & -2.83900000 & 0.18555900 \\
\hline $\mathrm{C}$ & 5.71216100 & -3.97304600 & 0.49392500 \\
\hline $\mathrm{H}$ & 6.09078600 & -3.80690700 & 1.50320900 \\
\hline $\mathrm{H}$ & 6.52091600 & -3.90995200 & -0.23513300 \\
\hline $\mathrm{O}$ & 0.25703700 & -1.33670100 & -0.83401700 \\
\hline $\mathrm{Cl}$ & 4.96504400 & -5.57097300 & 0.40267600 \\
\hline $\mathrm{C}$ & -0.76993200 & -0.74757700 & -0.44634100 \\
\hline $\mathrm{C}$ & -2.07231600 & -1.36435100 & -0.49941400 \\
\hline $\mathrm{C}$ & -0.64228800 & 0.62543100 & 0.19586100 \\
\hline $\mathrm{C}$ & -3.16154400 & -0.86140400 & 0.14285700 \\
\hline $\mathrm{C}$ & -3.14287700 & 0.46900700 & 0.89591500 \\
\hline $\mathrm{C}$ & -4.42323400 & -1.68384800 & 0.18440200 \\
\hline $\mathrm{C}$ & -4.49942400 & 1.21224400 & 0.67136900 \\
\hline $\mathrm{C}$ & -5.68795900 & -0.85329400 & -0.10046500 \\
\hline $\mathrm{H}$ & -4.53420400 & -2.10815300 & 1.19396100 \\
\hline $\mathrm{H}$ & -4.35809700 & -2.51901900 & -0.51637400 \\
\hline $\mathrm{C}$ & -5.75519700 & 0.34590100 & 0.83316300 \\
\hline $\mathrm{H}$ & -4.55513400 & 2.05946300 & 1.36216400 \\
\hline $\mathrm{H}$ & -4.49843300 & 1.63678800 & -0.34196200 \\
\hline $\mathrm{H}$ & -5.67866800 & -0.53466000 & -1.14850400 \\
\hline $\mathrm{H}$ & -6.64291400 & 0.94496500 & 0.60491000 \\
\hline $\mathrm{H}$ & -5.87028600 & -0.00394900 & 1.86629900 \\
\hline $\mathrm{H}$ & -2.12270500 & -2.32938000 & -0.99683400 \\
\hline $\mathrm{O}$ & -6.86122900 & -1.65114500 & 0.11995600 \\
\hline $\mathrm{C}$ & -7.20521700 & -2.51527900 & -0.88444800 \\
\hline $\mathrm{O}$ & -6.52617900 & -2.66820100 & -1.87482300 \\
\hline $\mathrm{C}$ & -8.50840100 & -3.20386600 & -0.57897300 \\
\hline $\mathrm{H}$ & -9.32384900 & -2.47237700 & -0.58997800 \\
\hline $\mathrm{H}$ & -8.69812500 & -3.97156600 & -1.32930800 \\
\hline $\mathrm{H}$ & -8.48333700 & -3.64800800 & 0.42072200 \\
\hline $\mathrm{C}$ & -0.70044100 & 3.60243400 & 0.44123900 \\
\hline $\mathrm{H}$ & -1.06109800 & 3.97460100 & -0.52570100 \\
\hline $\mathrm{H}$ & -0.54458300 & 4.48925300 & 1.06547500 \\
\hline $\mathrm{C}$ & 0.63175200 & 2.85379500 & 0.22163500 \\
\hline $\mathrm{C}$ & 0.31892700 & 1.56026500 & -0.57052300 \\
\hline $\mathrm{C}$ & 1.34631300 & 2.58677800 & 1.56118000 \\
\hline $\mathrm{H}$ & 2.19499500 & 1.89612400 & 1.45582800 \\
\hline $\mathrm{H}$ & 1.74339300 & 3.52295100 & 1.96226200 \\
\hline $\mathrm{H}$ & 0.67426100 & 2.16597900 & 2.31315600 \\
\hline $\mathrm{C}$ & 1.60839000 & 3.52986300 & -0.77889100 \\
\hline
\end{tabular}




$\begin{array}{lrrr}\mathrm{C} & 1.62236800 & 1.14542300 & -1.23443800 \\ \mathrm{H} & 2.32352100 & 0.13911600 & -0.62793200 \\ \mathrm{C} & 2.52828300 & 2.37361600 & -1.26386800 \\ \mathrm{H} & 1.03814500 & 3.93096300 & -1.62269900 \\ \mathrm{H} & 3.37009100 & 2.31278300 & -0.56026800 \\ \mathrm{H} & 2.95895300 & 2.55182000 & -2.25489200 \\ \mathrm{O} & 2.41508600 & 4.57008900 & -0.23871600 \\ \mathrm{C} & 1.98805200 & 5.86658900 & -0.47492500 \\ \mathrm{O} & 0.95790400 & 6.11039100 & -1.05128400 \\ \mathrm{C} & 2.97433000 & 6.85982100 & 0.07323200 \\ \mathrm{H} & 3.16478100 & 6.66615600 & 1.13355500 \\ \mathrm{H} & 3.92973800 & 6.76789600 & -0.45450100 \\ \mathrm{H} & 2.58052100 & 7.86776700 & -0.05712100 \\ \mathrm{C} & -2.96101900 & 0.15198200 & 2.40566800 \\ \mathrm{H} & -3.74249500 & -0.51943800 & 2.77004300 \\ \mathrm{H} & -1.99795000 & -0.32997600 & 2.60919400 \\ \mathrm{H} & -3.01991000 & 1.06866000 & 2.99969800 \\ \mathrm{H} & 1.50396500 & 0.59739800 & -2.17430600 \\ \mathrm{H} & -0.26610100 & 1.87582700 & -1.46029100 \\ \mathrm{H} & -0.22336900 & 0.43953700 & 1.19750400 \\ \mathrm{H} & -2.30242100 & 1.61838800 & -0.69101500 \\ \mathrm{C} & -1.99402100 & 1.36761800 & 0.33587000 \\ \mathrm{C} & -1.78084900 & 2.70560400 & 1.08560900 \\ \mathrm{H} & -1.51940900 & 2.50379400 & 2.13114700 \\ \mathrm{H} & -2.71787300 & 3.26947700 & 1.11124600\end{array}$

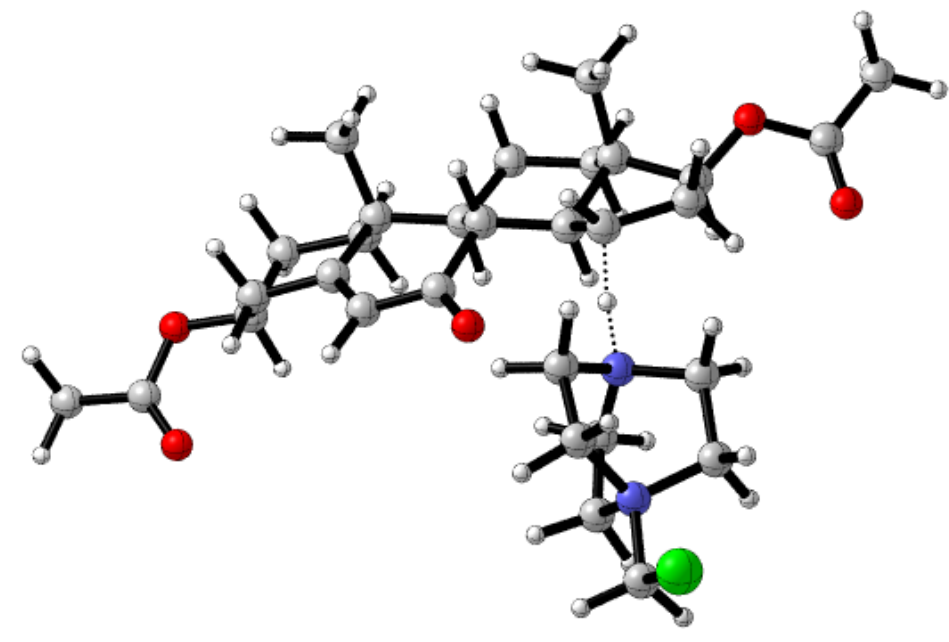

Figure S52. Transition state involving carbon C46 (bottom hydrogen) using SRD.

Zero-point correction $=$

Thermal correction to Energy=

Thermal correction to Enthalpy=
0.735076 (Hartree/Particle)

0.772541

0.773485 


$\begin{array}{ll}\text { Thermal correction to Gibbs Free Energy= } & 0.662865 \\ \text { Sum of electronic and zero-point Energies= } & -2115.384708 \\ \text { Sum of electronic and thermal Energies= } & -2115.347243 \\ \text { Sum of electronic and thermal Enthalpies= } & -2115.346299 \\ \text { Sum of electronic and thermal Free Energies= } & -2115.456919\end{array}$

Number of Imaginary Frequencies $=1$

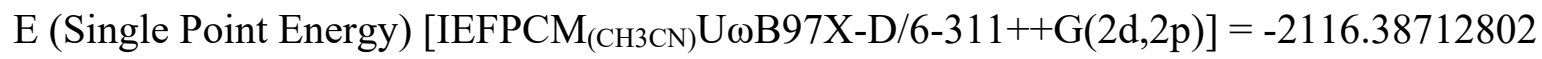

22

$\begin{array}{llll}\mathrm{C} & 2.84717500 & -1.99471800 & 1.32881100\end{array}$

C $\quad 2.32968400-1.74618500-1.04284100$

C $\quad 4.34608700 \quad-0.73282300-0.12674400$

$\begin{array}{llll}\text { C } & 3.79906400 & -3.19695700 & 1.10867300\end{array}$

$\begin{array}{llll}\mathrm{H} & 1.80444000 & -2.29206300 & 1.43607200\end{array}$

C $\quad 2.98292500 \quad-3.13990000-1.22216100$

$\mathrm{H} \quad 2.50695600-1.10614000-1.91003200$

$\mathrm{H} \quad \begin{array}{llll}1.25716000 & -1.83436900 & -0.86908900\end{array}$

$\mathrm{H} \quad 4.38032700 \quad 0.05297100 \quad-0.88362500$

$\mathrm{H} \quad 4.78894100 \quad-0.34338800 \quad 0.79236400$

C $\quad 5.09275600 \quad-1.99450100 \quad-0.63107900$

$\mathrm{H} \quad 3.30601100 \quad-4.14816700 \quad 1.32080900$

$\mathrm{H} \quad 4.70598300 \quad-3.13599800 \quad 1.71034900$

$\mathrm{H} \quad 3.28133000 \quad-3.31835600 \quad-2.25759100$

$\mathrm{H} \quad 2.32251200 \quad-3.94854800 \quad-0.90157600$

$\mathrm{H} \quad 5.26676700 \quad-1.96996800 \quad-1.70928000$

$\mathrm{H} \quad 6.04885800 \quad-2.13352100 \quad-0.12534400$

$\begin{array}{llll}\mathrm{N} & 2.92649800 & -1.08336100 & 0.15004700\end{array}$

$\mathrm{N} \quad 4.23507000 \quad-3.22153800 \quad-0.35276100$

C $\quad 4.97273400 \quad-4.50963500 \quad-0.70402400$

$\mathrm{H} \quad 5.27914800 \quad-4.43162300 \quad-1.74781500$

$\mathrm{H} \quad 4.26707000 \quad-5.33049100 \quad-0.57105000$

$\begin{array}{llll}\mathrm{O} & 0.05848900 & -1.15761600 & 0.77637800\end{array}$

$\begin{array}{llll}\mathrm{Cl} & 6.39654500 & -4.79013000 & 0.30433100\end{array}$

$\begin{array}{llll}\text { C } & -1.00532400 & -0.51406600 & 0.73884700\end{array}$

$\begin{array}{llll}\text { C } & -2.29182200 & -1.16741600 & 0.71487500\end{array}$

$\begin{array}{llll}\mathrm{C} & -0.98126600 & 1.01166100 & 0.74813200\end{array}$

$\begin{array}{llll}\mathrm{C} & -3.46776300 & -0.49900600 & 0.58658400\end{array}$

$\begin{array}{llll}\text { C } & -3.54718000 & 1.01133800 & 0.37157700\end{array}$

$\begin{array}{llll}\text { C } & -4.75989800 & -1.26837600 & 0.66513100\end{array}$

C $\quad-4.61033800 \quad 1.30861000 \quad-0.73599500$

$\begin{array}{llll}\mathrm{C} & -5.70794900 & -0.93261900 & -0.50163900\end{array}$

$\mathrm{H} \quad-5.27966000 \quad-1.00386300 \quad 1.59761900$

$\mathrm{H} \quad-4.56523200 \quad-2.34269000 \quad 0.69112500$

C $\quad-5.94544600 \quad 0.56833700 \quad-0.57668200$

$\mathrm{H} \quad-4.79940000 \quad 2.38636900 \quad-0.76235900$ 


\begin{tabular}{|c|c|c|c|}
\hline $\mathrm{H}$ & -4.17831000 & 1.04321500 & -1.71106300 \\
\hline $\mathrm{H}$ & -5.27166600 & -1.30651800 & -1.43437200 \\
\hline $\mathrm{H}$ & -6.59421000 & 0.79951200 & -1.42809600 \\
\hline $\mathrm{H}$ & -6.47968300 & 0.89751400 & 0.32256000 \\
\hline $\mathrm{H}$ & -2.28151500 & -2.24831800 & 0.82843800 \\
\hline $\mathrm{O}$ & -6.97653800 & -1.57592900 & -0.30717700 \\
\hline $\mathrm{C}$ & -7.04509700 & -2.89815500 & -0.65090000 \\
\hline $\mathrm{O}$ & -6.08278400 & -3.52330200 & -1.03844400 \\
\hline $\mathrm{C}$ & -8.44533500 & -3.42492000 & -0.48551600 \\
\hline $\mathrm{H}$ & -9.11894000 & -2.91475300 & -1.18244300 \\
\hline $\mathrm{H}$ & -8.45640200 & -4.49682700 & -0.68344400 \\
\hline $\mathrm{H}$ & -8.81187100 & -3.22390500 & 0.52592200 \\
\hline $\mathrm{C}$ & -0.72282900 & 3.61643800 & -0.71839800 \\
\hline $\mathrm{H}$ & -0.51033500 & 3.27001300 & -1.73982000 \\
\hline $\mathrm{H}$ & -0.74260500 & 4.71212900 & -0.75398600 \\
\hline $\mathrm{C}$ & 0.39817800 & 3.14483500 & 0.21934600 \\
\hline $\mathrm{C}$ & 0.34790400 & 1.55780200 & 0.23853300 \\
\hline $\mathrm{C}$ & 0.25976000 & 3.77974000 & 1.61749800 \\
\hline $\mathrm{H}$ & 1.02311400 & 3.43496200 & 2.32186600 \\
\hline $\mathrm{H}$ & 0.36704500 & 4.86446700 & 1.53012700 \\
\hline $\mathrm{H}$ & -0.71439900 & 3.58040600 & 2.06925400 \\
\hline $\mathrm{C}$ & 1.83951900 & 3.31075900 & -0.30762100 \\
\hline $\mathrm{C}$ & 1.65523500 & 1.21115400 & 0.90732400 \\
\hline $\mathrm{H}$ & 2.20375000 & 0.00637400 & 0.45567300 \\
\hline $\mathrm{C}$ & 2.66957800 & 2.28951200 & 0.51812500 \\
\hline $\mathrm{H}$ & 1.88271800 & 3.03973400 & -1.36852800 \\
\hline $\mathrm{H}$ & 3.08001800 & 2.76303200 & 1.41862200 \\
\hline $\mathrm{H}$ & 3.53313200 & 1.96042000 & -0.06803900 \\
\hline $\mathrm{O}$ & 2.30478200 & 4.64938600 & -0.17259500 \\
\hline $\mathrm{C}$ & 3.47215600 & 4.93518400 & -0.83789400 \\
\hline $\mathrm{O}$ & 4.10175200 & 4.07699200 & -1.41636500 \\
\hline $\mathrm{C}$ & 3.81468400 & 6.39434600 & -0.74062400 \\
\hline $\mathrm{H}$ & 4.80074200 & 6.56656500 & -1.17204500 \\
\hline $\mathrm{H}$ & 3.06971500 & 6.98572200 & -1.28444200 \\
\hline $\mathrm{H}$ & 3.79414800 & 6.72486100 & 0.30230700 \\
\hline $\mathrm{C}$ & -3.99449100 & 1.65620500 & 1.71323300 \\
\hline $\mathrm{H}$ & -4.93246700 & 1.22371700 & 2.06887300 \\
\hline $\mathrm{H}$ & -3.25346000 & 1.51446000 & 2.50733400 \\
\hline $\mathrm{H}$ & -4.16407800 & 2.72930500 & 1.58773400 \\
\hline $\mathrm{H}$ & 1.60589800 & 0.94789400 & 1.96884300 \\
\hline $\mathrm{H}$ & 0.42805800 & 1.28367600 & -0.82741200 \\
\hline $\mathrm{H}$ & -1.12063100 & 1.30963800 & 1.79819600 \\
\hline $\mathrm{H}$ & -2.00827200 & 1.12935900 & -1.11711700 \\
\hline $\mathrm{C}$ & -2.15954300 & 1.55581100 & -0.11214000 \\
\hline $\mathrm{C}$ & -2.10177600 & 3.09389900 & -0.26866300 \\
\hline $\mathrm{H}$ & -2.38173700 & 3.58141600 & 0.67033600 \\
\hline
\end{tabular}




$\begin{array}{lrrr}\mathrm{H} & -2.84611100 & 3.40815900 & -1.00652400 \\ \mathrm{H} & 3.14098600 & -1.43424600 & 2.21904400\end{array}$

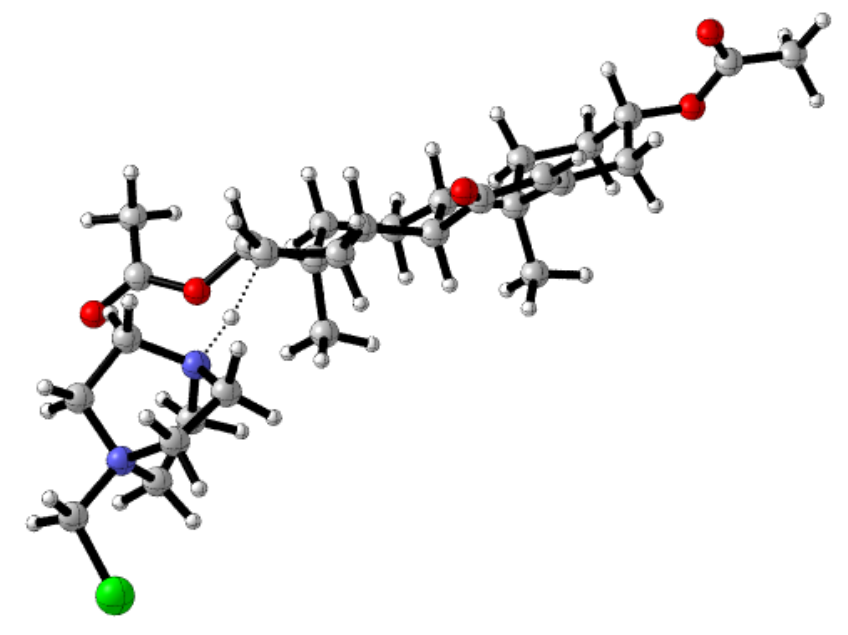

Figure S53. Transition state involving carbon C49 (top hydrogen) using SRD.

Zero-point correction $=$

Thermal correction to Energy=

Thermal correction to Enthalpy=

Thermal correction to Gibbs Free Energy=

Sum of electronic and zero-point Energies=

Sum of electronic and thermal Energies=

Sum of electronic and thermal Enthalpies=

Sum of electronic and thermal Free Energies=
0.734747 (Hartree/Particle)

0.772172

0.773116

0.662659

$-2115.360773$

$-2115.323348$

$-2115.322404$

$-2115.432861$

Number of Imaginary Frequencies $=1$

E (Single Point Energy) [IEFPCM $($ ICH3CN $) U \omega B$ B7X-D/6-311++G(2d,2p)] = -2116.37835672

\section{2}

$\begin{array}{lrrr}\mathrm{C} & 4.44363000 & -1.87644600 & -0.74027900 \\ \mathrm{C} & 5.15418400 & 0.36111100 & -1.42811700 \\ \mathrm{C} & 4.51594100 & -0.04116700 & 0.88399200 \\ \mathrm{C} & 5.95478000 & -2.18986600 & -0.63525500 \\ \mathrm{H} & 4.05790900 & -2.15749000 & -1.72311300 \\ \mathrm{C} & 6.60524700 & 0.17657900 & -0.92695300 \\ \mathrm{H} & 4.88015900 & 1.41526100 & -1.36676600 \\ \mathrm{H} & 5.02441000 & -0.00019300 & -2.45057200 \\ \mathrm{H} & 4.49920900 & 1.04790500 & 0.93973000 \\ \mathrm{H} & 3.71945600 & -0.43823200 & 1.51535200 \\ \mathrm{C} & 5.88601200 & -0.63254700 & 1.29157700 \\ \mathrm{H} & 6.42229700 & -2.32328800 & -1.61358600\end{array}$




\begin{tabular}{|c|c|c|c|}
\hline $\mathrm{H}$ & 6.14512800 & -3.07817700 & -0.03182000 \\
\hline $\mathrm{H}$ & 6.95335500 & 1.05027200 & -0.37143800 \\
\hline $\mathrm{H}$ & 7.29639400 & -0.02170400 & -1.74906000 \\
\hline $\mathrm{H}$ & 6.48747800 & 0.09531600 & 1.84055000 \\
\hline $\mathrm{H}$ & 5.79917600 & -1.53622700 & 1.89512700 \\
\hline $\mathrm{N}$ & 4.23358300 & -0.41260000 & -0.53903800 \\
\hline $\mathrm{N}$ & 6.65532800 & -1.01189600 & 0.02965500 \\
\hline $\mathrm{C}$ & 8.11879400 & -1.32412500 & 0.32505400 \\
\hline $\mathrm{H}$ & 8.57356900 & -0.40598500 & 0.69885700 \\
\hline $\mathrm{H}$ & 8.58084200 & -1.62672000 & -0.61545200 \\
\hline $\mathrm{O}$ & -1.78919100 & -1.95684500 & -1.91413900 \\
\hline $\mathrm{Cl}$ & 8.32250000 & -2.61078600 & 1.51888700 \\
\hline $\mathrm{C}$ & -2.43443500 & -1.35918000 & -1.05618900 \\
\hline $\mathrm{C}$ & -3.85588000 & -1.60341400 & -0.85036900 \\
\hline $\mathrm{C}$ & -1.76205000 & -0.34651800 & -0.11831900 \\
\hline $\mathrm{C}$ & -4.61446000 & -0.91406600 & 0.03370600 \\
\hline $\mathrm{C}$ & -4.08130400 & 0.25431500 & 0.86516100 \\
\hline $\mathrm{C}$ & -6.05620900 & -1.31558600 & 0.23266500 \\
\hline $\mathrm{C}$ & -5.13795400 & 1.40505000 & 0.85474200 \\
\hline $\mathrm{C}$ & -7.01447000 & -0.11347200 & 0.17227500 \\
\hline $\mathrm{H}$ & -6.16731300 & -1.77654400 & 1.22532900 \\
\hline $\mathrm{H}$ & -6.34837700 & -2.06232400 & -0.50890200 \\
\hline $\mathrm{C}$ & -6.57884900 & 0.96727000 & 1.15111200 \\
\hline $\mathrm{H}$ & -4.84037300 & 2.16835200 & 1.58146000 \\
\hline $\mathrm{H}$ & -5.11620500 & 1.88656900 & -0.13286800 \\
\hline $\mathrm{H}$ & -7.03978700 & 0.27324900 & -0.85211400 \\
\hline $\mathrm{H}$ & -7.24666000 & 1.83167100 & 1.07125300 \\
\hline $\mathrm{H}$ & -6.67708600 & 0.58914500 & 2.17591700 \\
\hline $\mathrm{H}$ & -4.28141500 & -2.40375300 & -1.44917600 \\
\hline $\mathrm{O}$ & -8.34352100 & -0.52349900 & 0.53492100 \\
\hline $\mathrm{C}$ & -9.08403900 & -1.12019100 & -0.44874300 \\
\hline $\mathrm{O}$ & -8.64406800 & -1.34866700 & -1.55263400 \\
\hline $\mathrm{C}$ & -10.47574900 & -1.42677600 & 0.03828200 \\
\hline $\mathrm{H}$ & -10.99992000 & -0.49567000 & 0.27877300 \\
\hline $\mathrm{H}$ & -11.02070000 & -1.96536100 & -0.73712200 \\
\hline $\mathrm{H}$ & -10.43585700 & -2.02397900 & 0.95463900 \\
\hline $\mathrm{C}$ & -0.77183600 & 2.44186500 & 0.38350400 \\
\hline $\mathrm{H}$ & -1.00899900 & 2.93628600 & -0.56930700 \\
\hline $\mathrm{H}$ & -0.32450200 & 3.20360100 & 1.03525800 \\
\hline $\mathrm{C}$ & 0.21675600 & 1.29204500 & 0.12158400 \\
\hline $\mathrm{C}$ & -0.49716800 & 0.22948500 & -0.76807400 \\
\hline $\mathrm{C}$ & 0.74289700 & 0.71499100 & 1.45171500 \\
\hline $\mathrm{H}$ & 1.40329800 & -0.14705600 & 1.30232300 \\
\hline $\mathrm{H}$ & 1.30500000 & 1.47754400 & 1.99927000 \\
\hline $\mathrm{H}$ & -0.07120500 & 0.38082800 & 2.09752900 \\
\hline $\mathrm{C}$ & 1.40031000 & 1.61905000 & -0.82580500 \\
\hline
\end{tabular}




$\begin{array}{lrrr}\mathrm{C} & 0.63383100 & -0.71033900 & -1.25149600 \\ \mathrm{C} & 1.75203900 & 0.28575700 & -1.49263300 \\ \mathrm{H} & 1.09044600 & 2.36120400 & -1.57112500 \\ \mathrm{H} & 3.06320100 & -0.12571600 & -0.86403200 \\ \mathrm{H} & 2.17247000 & 0.35644100 & -2.50290900 \\ \mathrm{O} & 2.61159500 & 2.07827000 & -0.16875800 \\ \mathrm{C} & 3.22894700 & 3.28230800 & -0.35852000 \\ \mathrm{O} & 4.41644300 & 3.31191300 & -0.09986100 \\ \mathrm{C} & 2.42457000 & 4.45701800 & -0.84569900 \\ \mathrm{H} & 3.01124200 & 5.36323400 & -0.69122900 \\ \mathrm{H} & 2.21428600 & 4.36548900 & -1.91829400 \\ \mathrm{H} & 1.46875700 & 4.53695800 & -0.32044000 \\ \mathrm{C} & -3.86613200 & -0.24711700 & 2.31834300 \\ \mathrm{H} & -4.78161400 & -0.68129400 & 2.72707700 \\ \mathrm{H} & -3.09231300 & -1.02046400 & 2.37606600 \\ \mathrm{H} & -3.58153600 & 0.57810400 & 2.97840600 \\ \mathrm{H} & 0.35842200 & -1.26656500 & -2.15353500 \\ \mathrm{H} & -1.50167600 & -0.90486300 & 0.79329200 \\ \mathrm{H} & -3.05142400 & 1.22626200 & -0.74423900 \\ \mathrm{C} & -2.75532200 & 0.79335100 & 0.22419000 \\ \mathrm{C} & -2.07740900 & 1.92886900 & 1.02805200 \\ \mathrm{H} & -1.87138000 & 1.59882500 & 2.05167900 \\ \mathrm{H} & -2.76725000 & 2.77330700 & 1.11660700 \\ \mathrm{H} & 0.86739700 & -1.47336800 & -0.49508500 \\ \mathrm{H} & 3.86500600 & -2.40735600 & 0.01870300 \\ \mathrm{H} & -0.83629900 & 0.76244900 & -1.66965300\end{array}$

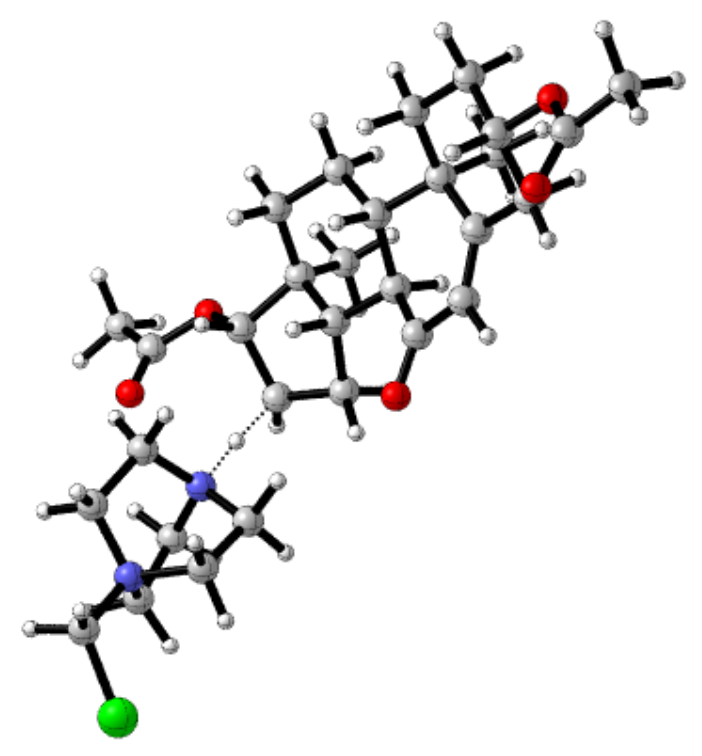

Figure S54. Transition state involving carbon C49 (bottom hydrogen) using SRD. 
Zero-point correction=

Thermal correction to Energy=

Thermal correction to Enthalpy=

Thermal correction to Gibbs Free Energy=

Sum of electronic and zero-point Energies $=$

Sum of electronic and thermal Energies=

Sum of electronic and thermal Enthalpies $=$

Sum of electronic and thermal Free Energies=

Number of Imaginary Frequencies $=1$

E (Single Point Energy) IEFPCM $\left._{(\mathrm{CH} 3 \mathrm{CN})} \mathrm{U} \omega \mathrm{B} 97 \mathrm{X}-\mathrm{D} / 6-311++\mathrm{G}(2 \mathrm{~d}, 2 \mathrm{p})\right]=-2116.38651245$

22

$\mathrm{C}$

C

C

$\mathrm{H}$

C

$\mathrm{H}$

$\mathrm{H}$

$\mathrm{H}$

$\mathrm{H}$

C

$\mathrm{H}$

$\mathrm{H}$

$\mathrm{H}$

$\mathrm{H}$

$\mathrm{H}$

$\mathrm{H}$

$\mathrm{N}$

$\mathrm{N}$

C

$\mathrm{H}$

$\mathrm{H}$

$\mathrm{O}$

$\mathrm{Cl}$

C

C

C

C

C

C

C

$\mathrm{C}$

$\mathrm{H}$
0.734898 (Hartree/Particle)

0.772212

0.773156

0.662864

$-2115.378715$

$-2115.341401$

$-2115.340457$

$-2115.450749$

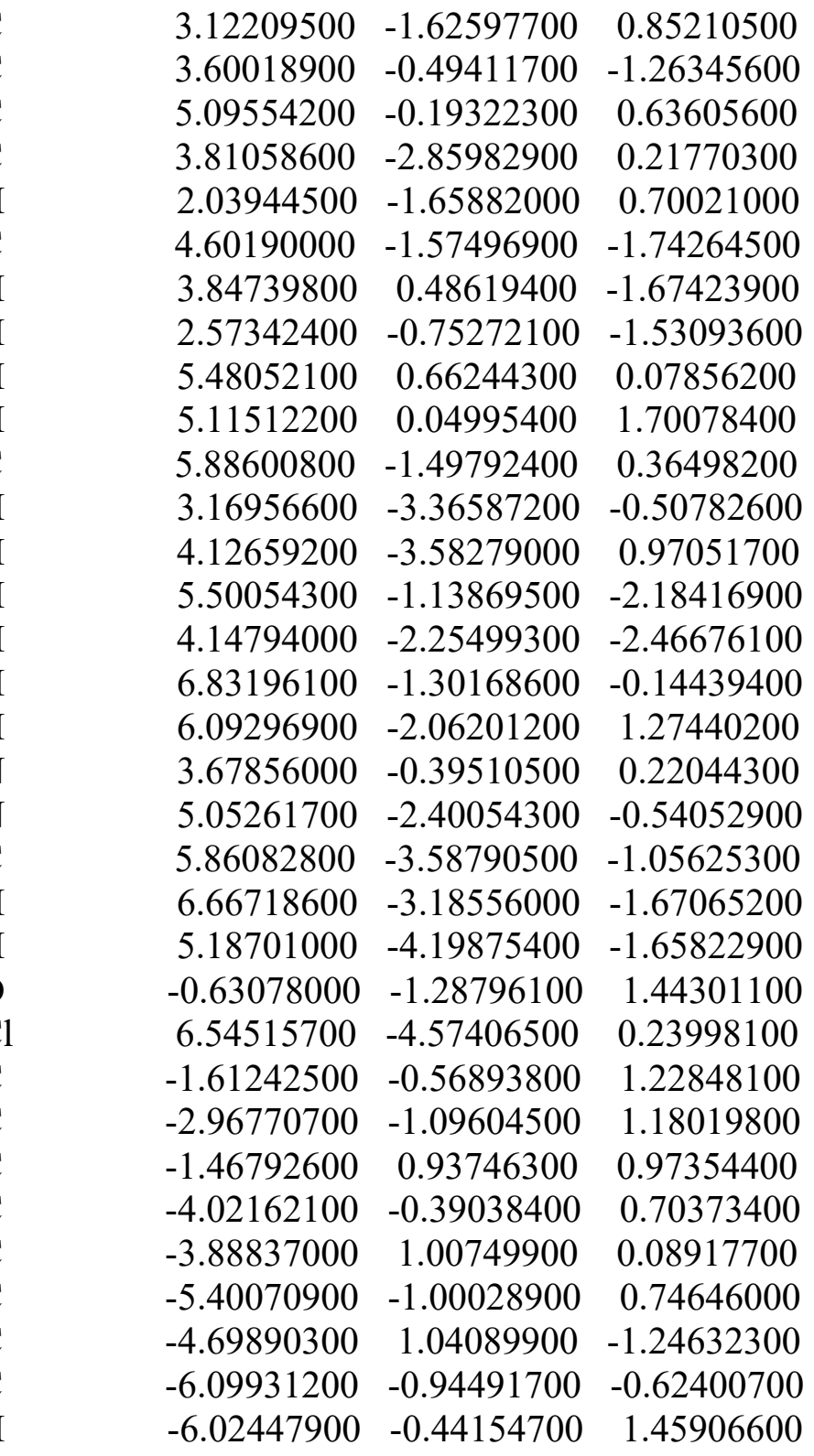




\begin{tabular}{|c|c|c|c|}
\hline $\mathrm{H}$ & -5.34950600 & -2.03391000 & 1.09601400 \\
\hline $\mathrm{C}$ & -6.12553100 & 0.48127000 & -1.15527400 \\
\hline $\mathrm{H}$ & -4.74187500 & 2.07300300 & -1.61020500 \\
\hline $\mathrm{H}$ & -4.14583000 & 0.46481700 & -2.00174000 \\
\hline $\mathrm{H}$ & -5.57525200 & -1.61040500 & -1.31837900 \\
\hline $\mathrm{H}$ & -6.58996300 & 0.49952900 & -2.14704900 \\
\hline $\mathrm{H}$ & -6.75432800 & 1.09989200 & -0.50389600 \\
\hline $\mathrm{H}$ & -3.09557800 & -2.11475800 & 1.53656400 \\
\hline $\mathrm{O}$ & -7.45815500 & -1.39717000 & -0.50401000 \\
\hline $\mathrm{C}$ & -7.65047200 & -2.75084200 & -0.51075500 \\
\hline $\mathrm{O}$ & -6.73654600 & -3.54301600 & -0.56719100 \\
\hline $\mathrm{C}$ & -9.11742700 & -3.08472200 & -0.44518800 \\
\hline $\mathrm{H}$ & -9.63129900 & -2.68723100 & -1.32678900 \\
\hline $\mathrm{H}$ & -9.24201100 & -4.16681300 & -0.40222100 \\
\hline $\mathrm{H}$ & -9.57373400 & -2.61913200 & 0.43415700 \\
\hline $\mathrm{C}$ & -0.59387300 & 2.94599100 & -1.09557900 \\
\hline $\mathrm{H}$ & -0.27896800 & 2.25013900 & -1.88766600 \\
\hline $\mathrm{H}$ & -0.45274800 & 3.95932500 & -1.48819000 \\
\hline $\mathrm{C}$ & 0.25865100 & 2.71512200 & 0.16103100 \\
\hline $\mathrm{C}$ & -0.01619900 & 1.28023700 & 0.68025600 \\
\hline $\mathrm{C}$ & 0.02903300 & 3.83424200 & 1.19702400 \\
\hline $\mathrm{H}$ & 0.67113600 & 3.74424900 & 2.07810400 \\
\hline $\mathrm{H}$ & 0.23908700 & 4.80719000 & 0.74363900 \\
\hline $\mathrm{H}$ & -1.00571900 & 3.84860800 & 1.54761800 \\
\hline $\mathrm{C}$ & 1.80029900 & 2.52307800 & -0.08671900 \\
\hline $\mathrm{C}$ & 1.05002400 & 1.07903400 & 1.76610200 \\
\hline $\mathrm{C}$ & 2.26080600 & 1.71390600 & 1.12783500 \\
\hline $\mathrm{H}$ & 1.90262700 & 1.95190900 & -1.01840200 \\
\hline $\mathrm{H}$ & 2.99689100 & 0.66123700 & 0.62008400 \\
\hline $\mathrm{O}$ & 2.52107500 & 3.74736500 & -0.24916500 \\
\hline $\mathrm{C}$ & 3.79727800 & 3.63675800 & -0.68565600 \\
\hline $\mathrm{O}$ & 4.32776100 & 2.54794000 & -0.86176700 \\
\hline $\mathrm{C}$ & 4.43435000 & 4.97394300 & -0.91931800 \\
\hline $\mathrm{H}$ & 3.97259500 & 5.44863800 & -1.79295100 \\
\hline $\mathrm{H}$ & 4.26366900 & 5.63538800 & -0.06499100 \\
\hline $\mathrm{H}$ & 5.50235300 & 4.85038600 & -1.09895200 \\
\hline $\mathrm{C}$ & -4.48016900 & 2.04124800 & 1.08453000 \\
\hline $\mathrm{H}$ & -5.51291300 & 1.79699400 & 1.34346500 \\
\hline $\mathrm{H}$ & -3.91689800 & 2.08183600 & 2.02290900 \\
\hline $\mathrm{H}$ & -4.48893800 & 3.04311100 & 0.64447100 \\
\hline $\mathrm{H}$ & 1.13874600 & 0.02090700 & 2.04365300 \\
\hline $\mathrm{H}$ & -1.81415800 & 1.46651900 & 1.87284000 \\
\hline $\mathrm{H}$ & -2.11033700 & 0.59278700 & -1.03380400 \\
\hline $\mathrm{C}$ & -2.38324100 & 1.29821700 & -0.23175600 \\
\hline $\mathrm{C}$ & -2.09173700 & 2.71349700 & -0.78369900 \\
\hline $\mathrm{H}$ & -2.43711200 & 3.47885200 & -0.0813380 \\
\hline
\end{tabular}




$\begin{array}{lrrr}\mathrm{H} & -2.66017100 & 2.86919100 & -1.70586100 \\ \mathrm{H} & 0.80999600 & 1.59698700 & 2.70712300 \\ \mathrm{H} & 3.31115900 & -1.56918800 & 1.92624000 \\ \mathrm{H} & 0.26891800 & 0.60743300 & -0.14806600 \\ \mathrm{H} & 3.02041000 & 2.17480800 & 1.76564700\end{array}$

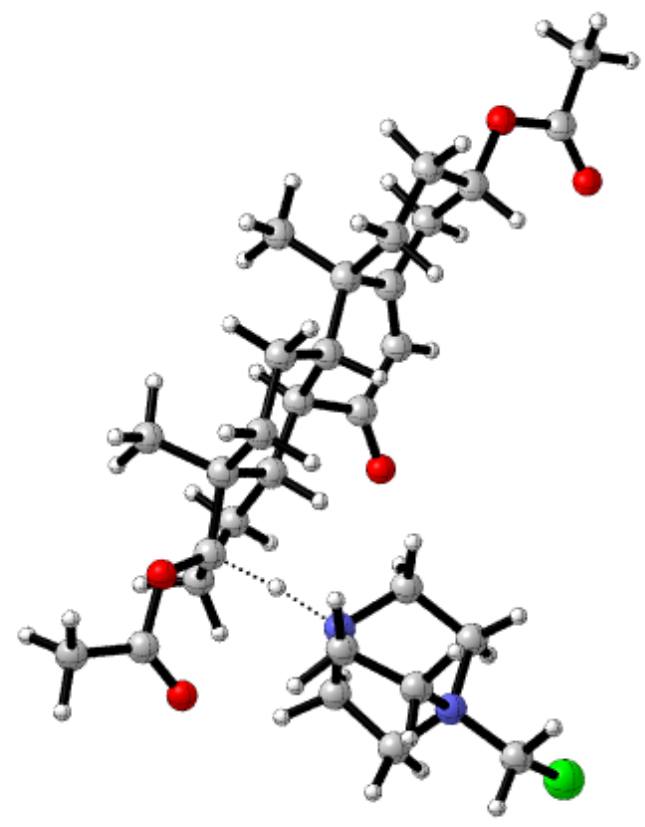

Figure S55. Transition state involving carbon C47 using SRD.

Zero-point correction $=$

Thermal correction to Energy=

Thermal correction to Enthalpy=

Thermal correction to Gibbs Free Energy=

Sum of electronic and zero-point Energies $=$

Sum of electronic and thermal Energies=

Sum of electronic and thermal Enthalpies=

Sum of electronic and thermal Free Energies=
0.735133 (Hartree/Particle)

0.772608

0.773552

0.663813

$-2115.367610$

$-2115.330136$

$-2115.329191$

$-2115.438931$

Number of Imaginary Frequencies $=1$

E (Single Point Energy) IEFPCM $\left._{(\mathrm{CH} 3 \mathrm{CN})} \mathrm{U} \omega \mathrm{B} 97 \mathrm{X}-\mathrm{D} / 6-311++\mathrm{G}(2 \mathrm{~d}, 2 \mathrm{p})\right]=-2116.38091986$

22

$\mathrm{C}$

$\mathrm{C}$

$\mathrm{C}$

$\mathrm{C}$

$\mathrm{H}$

$$
\begin{array}{rrr}
-2.06380200 & 1.42153700 & -0.22035300 \\
-3.89010600 & 0.41816000 & -1.49049500 \\
-4.10627400 & 0.75355300 & 0.91708600 \\
-2.68171800 & 2.84372800 & -0.24717100 \\
-1.45134700 & 1.23154400 & -1.10390900
\end{array}
$$




\begin{tabular}{|c|c|c|c|}
\hline $\mathrm{C}$ & -4.28674800 & 1.87267900 & -1.84771300 \\
\hline $\mathrm{H}$ & -4.76215800 & -0.22734700 & -1.38218600 \\
\hline $\mathrm{H}$ & -3.23812600 & 0.00064600 & -2.26106900 \\
\hline $\mathrm{H}$ & -4.78803500 & -0.08486100 & 1.05650100 \\
\hline $\mathrm{H}$ & -3.51638500 & 0.89790900 & 1.82437600 \\
\hline $\mathrm{C}$ & -4.88634600 & 2.03588000 & 0.53998900 \\
\hline $\mathrm{H}$ & -2.20421200 & 3.47947600 & -0.99598800 \\
\hline $\mathrm{H}$ & -2.61670900 & 3.34157000 & 0.72030200 \\
\hline $\mathrm{H}$ & -5.32018300 & 1.93085800 & -2.19661300 \\
\hline $\mathrm{H}$ & -3.63498900 & 2.31050000 & -2.60700000 \\
\hline $\mathrm{H}$ & -5.89604000 & 1.81885400 & 0.18339600 \\
\hline $\mathrm{H}$ & -4.95061300 & 2.73265700 & 1.37663000 \\
\hline $\mathrm{N}$ & -3.16231500 & 0.40958700 & -0.18962900 \\
\hline $\mathrm{N}$ & -4.16026800 & 2.73886400 & -0.60030700 \\
\hline $\mathrm{C}$ & -4.77468100 & 4.10301800 & -0.90160400 \\
\hline $\mathrm{H}$ & -5.83560800 & 3.94155600 & -1.09669000 \\
\hline $\mathrm{H}$ & -4.27517400 & 4.49189100 & -1.78985700 \\
\hline $\mathrm{O}$ & 0.76655500 & 0.66298100 & 2.33389700 \\
\hline $\mathrm{Cl}$ & -4.59028700 & 5.25142800 & 0.42736200 \\
\hline $\mathrm{C}$ & 1.60192600 & -0.04489400 & 1.77190800 \\
\hline $\mathrm{C}$ & 3.02094000 & 0.29653100 & 1.70233300 \\
\hline $\mathrm{C}$ & 1.20281700 & -1.32005200 & 1.01801600 \\
\hline $\mathrm{C}$ & 3.85234000 & -0.22372900 & 0.77064800 \\
\hline $\mathrm{C}$ & 3.37481900 & -1.16548500 & -0.34532700 \\
\hline $\mathrm{C}$ & 5.31221500 & 0.16040100 & 0.76857000 \\
\hline $\mathrm{C}$ & 3.94104600 & -0.64310100 & -1.70397600 \\
\hline $\mathrm{C}$ & 5.76617000 & 0.68374000 & -0.60480600 \\
\hline $\mathrm{H}$ & 5.92541800 & -0.72007900 & 1.00852500 \\
\hline $\mathrm{H}$ & 5.50703900 & 0.91323900 & 1.53597100 \\
\hline $\mathrm{C}$ & 5.44305900 & -0.32353100 & -1.69970500 \\
\hline $\mathrm{H}$ & 3.73574600 & -1.38306000 & -2.48558700 \\
\hline $\mathrm{H}$ & 3.39184800 & 0.26865000 & -1.98096400 \\
\hline $\mathrm{H}$ & 5.27565400 & 1.64307900 & -0.80132300 \\
\hline $\mathrm{H}$ & 5.73486700 & 0.08112700 & -2.67488100 \\
\hline $\mathrm{H}$ & 6.04016000 & -1.23031500 & -1.54651500 \\
\hline $\mathrm{H}$ & 3.37434700 & 1.03771900 & 2.41454700 \\
\hline $\mathrm{O}$ & 7.18894900 & 0.89574400 & -0.60869000 \\
\hline $\mathrm{C}$ & 7.62828800 & 2.07880300 & -0.08659200 \\
\hline $\mathrm{O}$ & 6.88020300 & 2.90466700 & 0.38825500 \\
\hline $\mathrm{C}$ & 9.12625200 & 2.19236100 & -0.19210800 \\
\hline $\mathrm{H}$ & 9.43316700 & 2.14398400 & -1.24202800 \\
\hline $\mathrm{H}$ & 9.45103500 & 3.13591300 & 0.24651700 \\
\hline $\mathrm{H}$ & 9.60478200 & 1.35502000 & 0.32605000 \\
\hline $\mathrm{C}$ & -0.36973200 & -2.03624700 & -1.45147600 \\
\hline $\mathrm{H}$ & -0.71111400 & -1.01818500 & -1.70258600 \\
\hline $\mathrm{H}$ & -0.76970000 & -2.70449300 & -2.2226740 \\
\hline
\end{tabular}




$\begin{array}{lrrr}\mathrm{C} & -0.88779300 & -2.42662700 & -0.05775000 \\ \mathrm{C} & -0.31255000 & -1.46262000 & 1.00056100 \\ \mathrm{C} & -0.56333300 & -3.92960900 & 0.21307900 \\ \mathrm{H} & -0.97574800 & -4.29003200 & 1.15825300 \\ \mathrm{H} & -0.96935800 & -4.55017600 & -0.59056800 \\ \mathrm{H} & 0.51689300 & -4.08354600 & 0.25201800 \\ \mathrm{C} & -2.37199500 & -2.23754100 & 0.26794600 \\ \mathrm{C} & -1.07229700 & -1.81389800 & 2.29665300 \\ \mathrm{C} & -2.49974600 & -2.20139100 & 1.80817700 \\ \mathrm{H} & -2.70578700 & -0.89867200 & -0.00692400 \\ \mathrm{O} & -3.21056900 & -3.04800000 & -0.47548200 \\ \mathrm{C} & -4.58856600 & -3.02385300 & -0.31023700 \\ \mathrm{O} & -5.14209600 & -2.05764800 & 0.16384800 \\ \mathrm{C} & -5.22156100 & -4.27752300 & -0.82333400 \\ \mathrm{H} & -4.95103400 & -5.11598100 & -0.17024500 \\ \mathrm{H} & -6.30558300 & -4.16376500 & -0.83628700 \\ \mathrm{H} & -4.84597400 & -4.51598900 & -1.82330200 \\ \mathrm{C} & 3.92091800 & -2.59125000 & -0.07099200 \\ \mathrm{H} & 5.00945100 & -2.58724600 & 0.01951200 \\ \mathrm{H} & 3.52745100 & -3.01531700 & 0.85865300 \\ \mathrm{H} & 3.67247400 & -3.27071600 & -0.89267900 \\ \mathrm{H} & -1.07543900 & -0.97809900 & 2.99680300 \\ \mathrm{H} & 1.66764500 & -2.17994500 & 1.51718000 \\ \mathrm{H} & 1.54585900 & -0.11472700 & -0.71578900 \\ \mathrm{C} & 1.80843200 & -1.14200700 & -0.40950500 \\ \mathrm{C} & 1.17500000 & -2.06413700 & -1.47555100 \\ \mathrm{H} & 1.51736500 & -3.09640300 & -1.35760200 \\ \mathrm{H} & 1.50860100 & -1.74928900 & -2.46946700 \\ \mathrm{H} & -0.60788000 & -2.66350600 & 2.80756000 \\ \mathrm{H} & -1.43245000 & 1.30657900 & 0.66445600 \\ \mathrm{H} & -0.66769900 & -0.46858800 & 0.70596000 \\ \mathrm{H} & -2.80948700 & -3.20093500 & 2.14114900 \\ \mathrm{H} & -3.27618600 & -1.52427300 & 2.17051200\end{array}$




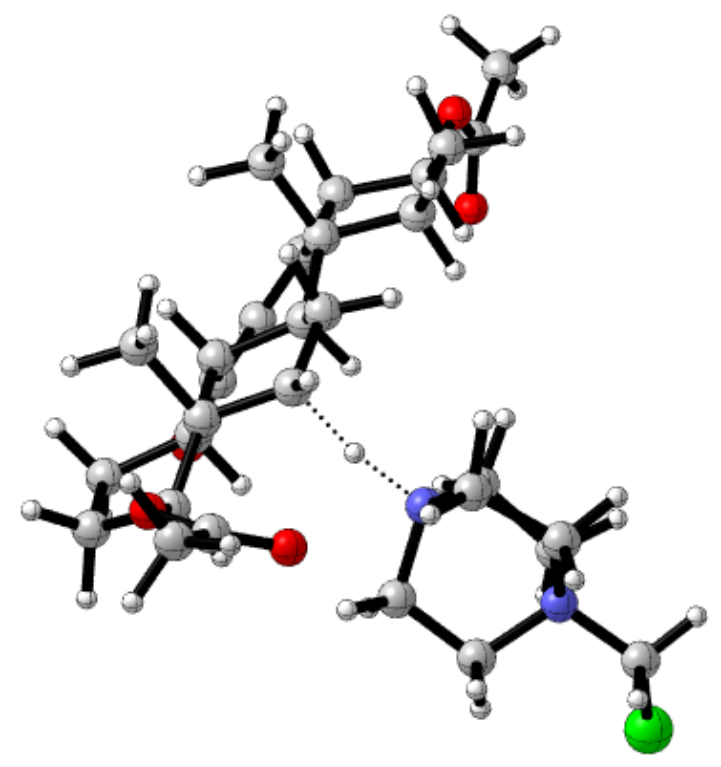

Figure S56. Transition state involving carbon C38 (bottom hydrogen) using SRD.

Zero-point correction $=$

Thermal correction to Energy=

Thermal correction to Enthalpy=

Thermal correction to Gibbs Free Energy=

Sum of electronic and zero-point Energies $=$

Sum of electronic and thermal Energies=

Sum of electronic and thermal Enthalpies=

Sum of electronic and thermal Free Energies=
0.734768 (Hartree/Particle)

0.772323

0.773267

0.662688

$-2115.363414$

$-2115.325859$

$-2115.324915$

$-2115.435494$

Number of Imaginary Frequencies $=1$

E (Single Point Energy) IEFPCM $_{(\mathrm{CH} 3 \mathrm{CN})} \mathrm{U \omega B}$ B7X-D/6-311++G(2d,2p)] $=-2116.38545836$

22

$\mathrm{C}$

$\mathrm{C}$

$\mathrm{C}$

$\mathrm{C}$

$\mathrm{H}$

C

$\mathrm{H}$

$\mathrm{H}$

$\mathrm{H}$

$\mathrm{H}$

C

$\mathrm{H}$

$\begin{array}{rrr}-1.16859300 & 2.00788500 & -0.18132000 \\ -2.98681300 & 1.34516100 & -1.67417400 \\ -3.20716100 & 1.02735400 & 0.73391600 \\ -1.79847200 & 3.38942200 & 0.13813400 \\ -0.54221500 & 2.04802200 & -1.07472700 \\ -3.43743800 & 2.82770000 & -1.62437100 \\ -3.82657800 & 0.65221900 & -1.75095800 \\ -2.31580300 & 1.18171000 & -2.52048900 \\ -3.89334900 & 0.19141200 & 0.59465800 \\ -2.63082300 & 0.88596500 & 1.65065300 \\ -3.97714200 & 2.37041000 & 0.74139400 \\ -1.34618300 & 4.18934200 & -0.45207500\end{array}$




\begin{tabular}{|c|c|c|c|}
\hline $\mathrm{H}$ & -1.71607600 & 3.64986300 & 1.19330900 \\
\hline $\mathrm{H}$ & -4.48633700 & 2.93564000 & -1.90895000 \\
\hline $\mathrm{H}$ & -2.83182000 & 3.47207100 & -2.26556100 \\
\hline $\mathrm{H}$ & -5.00115000 & 2.25878100 & 0.37750600 \\
\hline $\mathrm{H}$ & -4.00288600 & 2.82291000 & 1.73336400 \\
\hline $\mathrm{N}$ & -2.25509000 & 1.01808500 & -0.41565900 \\
\hline $\mathrm{N}$ & -3.28369400 & 3.34891400 & -0.19900500 \\
\hline $\mathrm{C}$ & -3.91843500 & 4.73637300 & -0.13029600 \\
\hline $\mathrm{H}$ & -4.98071600 & 4.61345000 & -0.34470000 \\
\hline $\mathrm{H}$ & -3.43986800 & 5.34771400 & -0.89639900 \\
\hline $\mathrm{O}$ & 1.38774000 & -1.81091300 & 3.16612400 \\
\hline $\mathrm{Cl}$ & -3.72308900 & 5.50870700 & 1.44594200 \\
\hline G & 1.82601100 & -1.72828400 & 2.02862300 \\
\hline $\mathrm{C}$ & 3.17412500 & -1.22814200 & 1.73949900 \\
\hline $\mathrm{C}$ & 0.98589100 & -2.14281600 & 0.80699400 \\
\hline $\mathrm{C}$ & 3.61568400 & -0.92709900 & 0.49998300 \\
\hline $\mathrm{C}$ & 2.72025500 & -1.01242800 & -0.74382200 \\
\hline $\mathrm{C}$ & 5.04496800 & -0.48291700 & 0.29552000 \\
\hline $\mathrm{C}$ & 2.93856100 & 0.26635900 & -1.61272400 \\
\hline $\mathrm{C}$ & 5.14326800 & 0.80108800 & -0.54339900 \\
\hline $\mathrm{H}$ & 5.60222500 & -1.26979600 & -0.23363400 \\
\hline $\mathrm{H}$ & 5.53396200 & -0.33398900 & 1.26129800 \\
\hline $\mathrm{C}$ & 4.40712100 & 0.63633100 & -1.86593700 \\
\hline $\mathrm{H}$ & 2.43285500 & 0.13823100 & -2.57736600 \\
\hline $\mathrm{H}$ & 2.45236400 & 1.11557100 & -1.10642000 \\
\hline $\mathrm{H}$ & 4.72805900 & 1.63646000 & 0.03085800 \\
\hline $\mathrm{H}$ & 4.45915400 & 1.56775900 & -2.44045700 \\
\hline $\mathrm{H}$ & 4.90745900 & -0.13005300 & -2.46947500 \\
\hline $\mathrm{H}$ & 3.81983400 & -1.10831900 & 2.60558500 \\
\hline $\mathrm{O}$ & 6.51717000 & 1.09455600 & -0.83736800 \\
\hline $\mathrm{C}$ & 7.20318900 & 1.79658800 & 0.11949000 \\
\hline $\mathrm{O}$ & 6.69616200 & 2.14638100 & 1.16079500 \\
\hline $\mathrm{C}$ & 8.62274200 & 2.04731800 & -0.31345700 \\
\hline $\mathrm{H}$ & 9.13268200 & 2.64333100 & 0.44346200 \\
\hline $\mathrm{H}$ & 9.14627400 & 1.09486300 & -0.44823000 \\
\hline $\mathrm{H}$ & 8.64065200 & 2.56718500 & -1.27654300 \\
\hline $\mathrm{C}$ & -1.22582500 & -1.43324500 & -1.07818900 \\
\hline $\mathrm{H}$ & -1.73669600 & -0.21934300 & -0.59331000 \\
\hline $\mathrm{H}$ & -1.92157500 & -1.44816600 & -1.92765100 \\
\hline $\mathrm{C}$ & -1.40102700 & -2.54523700 & -0.07650500 \\
\hline $\mathrm{C}$ & -0.49327200 & -2.30622900 & 1.16175700 \\
\hline $\mathrm{C}$ & -1.06287700 & -3.87197800 & -0.83781900 \\
\hline $\mathrm{H}$ & -1.22050000 & -4.73412100 & -0.18576000 \\
\hline $\mathrm{H}$ & -1.71269300 & -3.99596800 & -1.70866200 \\
\hline $\mathrm{H}$ & -0.02627000 & -3.89052900 & -1.17891000 \\
\hline $\mathrm{C}$ & -2.76175300 & -2.72050500 & 0.6447710 \\
\hline
\end{tabular}




$\begin{array}{lrrr}\mathrm{C} & -0.92994200 & -3.41071100 & 2.15097300 \\ \mathrm{C} & -2.44477400 & -3.62795400 & 1.85310900 \\ \mathrm{O} & -3.80520100 & -3.30671400 & -0.16399100 \\ \mathrm{C} & -4.61525400 & -2.49514300 & -0.85861500 \\ \mathrm{O} & -4.47253100 & -1.27527300 & -0.88982400 \\ \mathrm{C} & -5.70809100 & -3.25141200 & -1.55782000 \\ \mathrm{H} & -6.21044600 & -2.60423200 & -2.27704800 \\ \mathrm{H} & -5.30836500 & -4.14008700 & -2.05376300 \\ \mathrm{H} & -6.43689900 & -3.59759400 & -0.81533600 \\ \mathrm{C} & 3.12609700 & -2.26711300 & -1.56074700 \\ \mathrm{H} & 4.18656300 & -2.23769400 & -1.82124000 \\ \mathrm{H} & 2.96321900 & -3.19432000 & -1.00305700 \\ \mathrm{H} & 2.57392500 & -2.32769600 & -2.50567800 \\ \mathrm{H} & -0.73684900 & -3.11134000 & 3.17995600 \\ \mathrm{H} & 1.38633200 & -3.10714000 & 0.46569000 \\ \mathrm{H} & 1.03883400 & -0.09583200 & 0.20813300 \\ \mathrm{C} & 1.22149300 & -1.06504600 & -0.28682900 \\ \mathrm{C} & 0.21131200 & -1.14599100 & -1.46348400 \\ \mathrm{H} & 0.50464700 & -1.96466700 & -2.14109100 \\ \mathrm{H} & 0.26878700 & -0.24526600 & -2.08726500 \\ \mathrm{H} & -0.36200600 & -4.33075200 & 1.97807000 \\ \mathrm{H} & -0.55444000 & 1.66556700 & 0.65362700 \\ \mathrm{H} & -0.81550900 & -1.34540700 & 1.60136500 \\ \mathrm{H} & -2.66934500 & -4.66792000 & 1.60102500 \\ \mathrm{H} & -3.07803800 & -3.36869800 & 2.70548900 \\ \mathrm{H} & -3.12019200 & -1.74888000 & 0.98853100\end{array}$

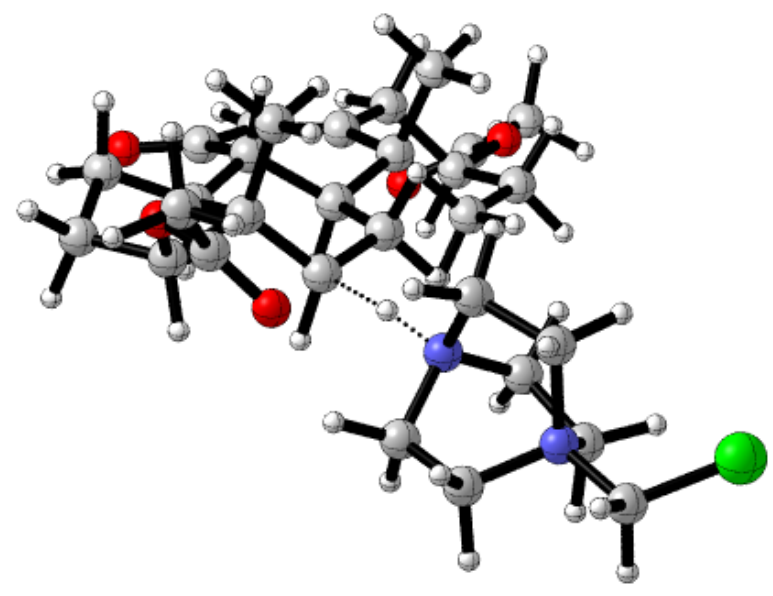

Figure S57. Transition state involving carbon C38 (yop hydrogen) using SRD.

Zero-point correction=

Thermal correction to Energy=

Thermal correction to Enthalpy=
0.735889 (Hartree/Particle)

0.773292

0.774237 


$\begin{array}{ll}\text { Thermal correction to Gibbs Free Energy= } & 0.663310 \\ \text { Sum of electronic and zero-point Energies }= & -2115.366916 \\ \text { Sum of electronic and thermal Energies= } & -2115.329513 \\ \text { Sum of electronic and thermal Enthalpies= } & -2115.328569 \\ \text { Sum of electronic and thermal Free Energies= } & -2115.439495\end{array}$

Number of Imaginary Frequencies $=1$

E (Single Point Energy) [IEFPCM $\left.{ }_{(\mathrm{CH} 3 \mathrm{CN})} \mathrm{U} \omega \mathrm{B} 97 \mathrm{X}-\mathrm{D} / 6-311++\mathrm{G}(2 \mathrm{~d}, 2 \mathrm{p})\right]=-2116.38509348$

$\begin{array}{lrrr}2 \mathrm{C} & & & \\ \mathrm{C} & -3.79852500 & -0.72513500 & 0.92527700 \\ \mathrm{C} & -3.79292600 & -0.50204300 & -1.51887000 \\ \mathrm{C} & -2.39605700 & -2.18390500 & -0.44158800 \\ \mathrm{C} & -4.80407000 & -1.90053100 & 0.93331200 \\ \mathrm{H} & -4.28924600 & 0.24880300 & 0.91100800 \\ \mathrm{C} & -5.08065700 & -1.35124700 & -1.45832400 \\ \mathrm{H} & -3.19486600 & -0.73346700 & -2.40300700 \\ \mathrm{H} & -4.02492900 & 0.56280900 & -1.49810100 \\ \mathrm{H} & -1.64440900 & -2.18659800 & -1.23354200 \\ \mathrm{H} & -1.90554000 & -2.44093800 & 0.49937200 \\ \mathrm{C} & -3.54300800 & -3.16629100 & -0.78093400 \\ \mathrm{H} & -5.80909500 & -1.56805900 & 1.20216700 \\ \mathrm{H} & -4.51485900 & -2.70611400 & 1.60847900 \\ \mathrm{H} & -5.32672100 & -1.78384100 & -2.43066300 \\ \mathrm{H} & -5.93615900 & -0.77393400 & -1.10102400 \\ \mathrm{H} & -3.56439900 & -3.43162000 & -1.84044300 \\ \mathrm{H} & -3.48180900 & -4.08212700 & -0.19187300 \\ \mathrm{~N} & -2.95933600 & -0.80682500 & -0.31222200 \\ \mathrm{~N} & -4.87493500 & -2.49736600 & -0.46864300 \\ \mathrm{C} & -6.03978300 & -3.47108100 & -0.61706500 \\ \mathrm{H} & -5.98963400 & -3.88293200 & -1.62576100 \\ \mathrm{H} & -6.95706700 & -2.89671300 & -0.48196900 \\ \mathrm{O} & 3.32013400 & 3.73937000 & -0.83755800 \\ \mathrm{Cl} & -5.99251100 & -4.79004500 & 0.55771400 \\ \mathrm{C} & 3.18897400 & 2.63815700 & -0.33552600 \\ \mathrm{C} & 4.32426200 & 1.72919000 & -0.14394900 \\ \mathrm{C} & 1.81644500 & 2.12333100 & 0.19062500 \\ \mathrm{C} & 4.22281900 & 0.46160300 & 0.30289000 \\ \mathrm{C} & 2.87680500 & -0.21231200 & 0.60720800 \\ \mathrm{C} & 5.47406100 & -0.35336700 & 0.53949000 \\ \mathrm{C} & 2.89368500 & -1.65851000 & 0.02098200 \\ \mathrm{C} & 5.39898100 & -1.74712800 & -0.10298000 \\ \mathrm{H} & 5.62000700 & -0.49346600 & 1.62074700 \\ \mathrm{H} & 6.34699300 & 0.18266800 & 0.16051500 \\ \mathrm{C} & 4.14611600 & -2.48118700 & 0.35559700 \\ \mathrm{H} & 2.00882800 & -2.20038600 & 0.37847200\end{array}$




\begin{tabular}{|c|c|c|c|}
\hline $\mathrm{H}$ & 2.80485600 & -1.59034100 & -1.07292700 \\
\hline $\mathrm{H}$ & 5.41223600 & -1.64129800 & -1.19307400 \\
\hline $\mathrm{H}$ & 4.08703500 & -3.45799900 & -0.13692800 \\
\hline $\mathrm{H}$ & 4.21501700 & -2.67653900 & 1.43262800 \\
\hline $\mathrm{H}$ & 5.29348800 & 2.16136700 & -0.37790200 \\
\hline $\mathrm{O}$ & 6.53205100 & -2.53724300 & 0.29074600 \\
\hline $\mathrm{C}$ & 7.68582800 & -2.33482800 & -0.42172400 \\
\hline $\mathrm{O}$ & 7.76798300 & -1.52164100 & -1.31283100 \\
\hline $\mathrm{C}$ & 8.78215300 & -3.24649800 & 0.06170500 \\
\hline $\mathrm{H}$ & 8.47707700 & -4.29276100 & -0.04312100 \\
\hline $\mathrm{H}$ & 9.68672600 & -3.06349200 & -0.51832300 \\
\hline $\mathrm{H}$ & 8.97897500 & -3.06910300 & 1.12409300 \\
\hline $\mathrm{C}$ & -0.77832500 & 0.89854700 & -0.43258000 \\
\hline $\mathrm{H}$ & -2.05215000 & 0.02303700 & -0.25722000 \\
\hline $\mathrm{H}$ & -0.83301600 & 0.84067000 & -1.52981000 \\
\hline $\mathrm{C}$ & -0.78646500 & 2.31665600 & 0.07267200 \\
\hline $\mathrm{C}$ & 0.64494500 & 2.85255300 & -0.45643300 \\
\hline $\mathrm{C}$ & -0.91195500 & 2.42217600 & 1.60489000 \\
\hline $\mathrm{H}$ & -0.85105200 & 3.45834000 & 1.94309100 \\
\hline $\mathrm{H}$ & -1.88007300 & 2.03158300 & 1.93743500 \\
\hline $\mathrm{H}$ & -0.13559700 & 1.86030500 & 2.12653500 \\
\hline $\mathrm{C}$ & -1.75020700 & 3.34013500 & -0.63531900 \\
\hline $\mathrm{C}$ & 0.48003200 & 4.37137400 & -0.37275600 \\
\hline $\mathrm{C}$ & -0.89646900 & 4.57435500 & -1.02520500 \\
\hline $\mathrm{O}$ & -2.79810000 & 3.84875600 & 0.24159600 \\
\hline $\mathrm{C}$ & -3.94087500 & 3.18784500 & 0.39474300 \\
\hline $\mathrm{O}$ & -4.14368900 & 2.07162400 & -0.08004900 \\
\hline $\mathrm{C}$ & -4.94341000 & 3.97393300 & 1.19401400 \\
\hline $\mathrm{H}$ & -4.47634900 & 4.40715300 & 2.08272100 \\
\hline $\mathrm{H}$ & -5.31253900 & 4.80788900 & 0.58562800 \\
\hline $\mathrm{H}$ & -5.78472600 & 3.33959700 & 1.47468500 \\
\hline $\mathrm{C}$ & 2.69588900 & -0.26855400 & 2.14576000 \\
\hline $\mathrm{H}$ & 3.54139800 & -0.77037500 & 2.62194900 \\
\hline $\mathrm{H}$ & 2.62884200 & 0.72997100 & 2.58897500 \\
\hline $\mathrm{H}$ & 1.79823500 & -0.83304100 & 2.42307800 \\
\hline $\mathrm{H}$ & 1.28938000 & 4.87252500 & -0.90607400 \\
\hline $\mathrm{H}$ & 1.83217800 & 2.31065300 & 1.27261300 \\
\hline $\mathrm{H}$ & 1.91417500 & 0.49513400 & -1.17820200 \\
\hline $\mathrm{C}$ & 1.74658200 & 0.60919500 & -0.09747200 \\
\hline $\mathrm{C}$ & 0.29742700 & 0.02307900 & 0.16349200 \\
\hline $\mathrm{H}$ & 0.15194100 & -0.08838600 & 1.24234300 \\
\hline $\mathrm{H}$ & 0.27680200 & -0.97996100 & -0.27656500 \\
\hline $\mathrm{H}$ & 0.49754000 & 4.72298300 & 0.66473000 \\
\hline $\mathrm{H}$ & -3.13618800 & -0.77870100 & 1.79218000 \\
\hline $\mathrm{H}$ & 0.68288600 & 2.61398700 & -1.53036000 \\
\hline $\mathrm{H}$ & -1.39735800 & 5.49486200 & -0.7178400 \\
\hline
\end{tabular}




$$
\begin{array}{llll}
\mathrm{H} & -0.79021800 & 4.61140300 & -2.11451100 \\
\mathrm{H} & -2.24467800 & 2.88252600 & -1.49613000
\end{array}
$$

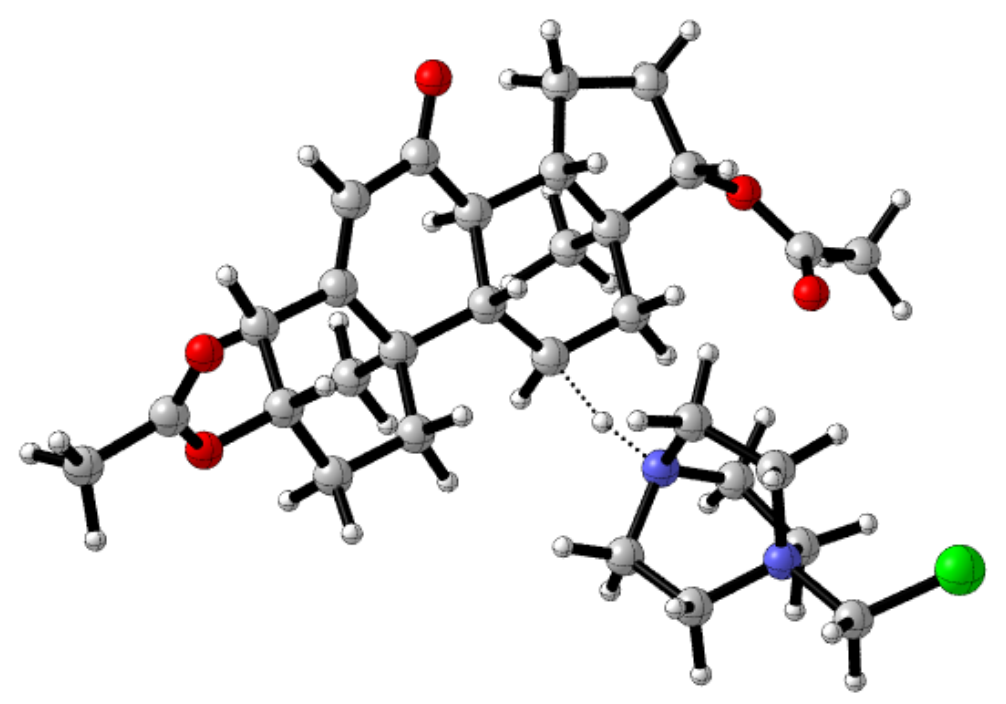

Figure S58. Transition state involving carbon C32 (bottom hydrogen) using SRD.

Zero-point correction $=$

Thermal correction to Energy=

Thermal correction to Enthalpy=

Thermal correction to Gibbs Free Energy=

Sum of electronic and zero-point Energies=

Sum of electronic and thermal Energies $=$

Sum of electronic and thermal Enthalpies=

Sum of electronic and thermal Free Energies=
0.735258 (Hartree/Particle)

0.772910

0.773855

0.662589

$-2115.352815$

$-2115.315162$

$-2115.314218$

$-2115.425484$

Number of Imaginary Frequencies $=1$

E (Single Point Energy) [IEFPCM $\left(\mathrm{ICH}_{3 \mathrm{CN})} \mathrm{U} \omega \mathrm{B} 97 \mathrm{X}-\mathrm{D} / 6-311++\mathrm{G}(2 \mathrm{~d}, 2 \mathrm{p})\right]=-2116.38215268$

$\begin{array}{cccc}22 & & & \\ \mathrm{C} & 1.23221200 & 2.01429600 & -0.97159700 \\ \mathrm{C} & 0.64992100 & 2.89509800 & 1.22627500 \\ \mathrm{C} & 2.73420300 & 1.65583300 & 0.92805500 \\ \mathrm{C} & 2.15225600 & 3.20593300 & -1.33226000 \\ \mathrm{H} & 0.18960500 & 2.20929300 & -1.23135400 \\ \mathrm{C} & 1.24742800 & 4.23308600 & 0.72349000 \\ \mathrm{H} & 0.81953700 & 2.75202700 & 2.29553300 \\ \mathrm{H} & -0.42432900 & 2.85934900 & 1.04139600 \\ \mathrm{H} & 2.75861900 & 1.31576900 & 1.96533200 \\ \mathrm{H} & 3.23260100 & 0.90093900 & 0.31612300\end{array}$




\begin{tabular}{|c|c|c|c|}
\hline $\mathrm{C}$ & 3.40785200 & 3.04283100 & 0.79867700 \\
\hline $\mathrm{H}$ & 1.65541900 & 3.91655000 & -1.99639300 \\
\hline $\mathrm{H}$ & 3.08291000 & 2.88623700 & -1.80106900 \\
\hline $\mathrm{H}$ & 1.49777400 & 4.89791400 & 1.55306300 \\
\hline $\mathrm{H}$ & 0.57234100 & 4.75962600 & 0.04522700 \\
\hline $\mathrm{H}$ & 3.53036800 & 3.54311700 & 1.76222500 \\
\hline $\mathrm{H}$ & 4.38237400 & 2.97093700 & 0.31423900 \\
\hline $\mathrm{N}$ & 1.29888000 & 1.76258400 & 0.50074000 \\
\hline $\mathrm{N}$ & 2.52668100 & 3.94712500 & -0.05387900 \\
\hline $\mathrm{C}$ & 3.21323800 & 5.28030400 & -0.35069100 \\
\hline $\mathrm{H}$ & 3.48492500 & 5.72113400 & 0.60930600 \\
\hline $\mathrm{H}$ & 2.48669200 & 5.90678400 & -0.86983200 \\
\hline $\mathrm{O}$ & -1.92751000 & -3.78907200 & -2.07076700 \\
\hline $\mathrm{Cl}$ & 4.65915400 & 5.10361700 & -1.34743900 \\
\hline $\mathrm{C}$ & -2.06578800 & -2.97097600 & -1.17923200 \\
\hline $\mathrm{C}$ & -3.31314000 & -2.22512700 & -0.99158500 \\
\hline $\mathrm{C}$ & -0.91988600 & -2.71318900 & -0.17197700 \\
\hline $\mathrm{C}$ & -3.47692000 & -1.24288400 & -0.08379600 \\
\hline $\mathrm{C}$ & -2.33067300 & -0.74791400 & 0.80678800 \\
\hline $\mathrm{C}$ & -4.83688100 & -0.61249800 & 0.10990900 \\
\hline $\mathrm{C}$ & -2.39050500 & 0.80707000 & 0.89471600 \\
\hline $\mathrm{C}$ & -4.77935900 & 0.92169100 & 0.14655200 \\
\hline $\mathrm{H}$ & -5.26580000 & -0.95245600 & 1.06405300 \\
\hline $\mathrm{H}$ & -5.51597000 & -0.93809900 & -0.68181500 \\
\hline $\mathrm{C}$ & -3.77681400 & 1.39653200 & 1.19118200 \\
\hline $\mathrm{H}$ & -1.68457900 & 1.12954100 & 1.67326200 \\
\hline $\mathrm{H}$ & -2.03891500 & 1.21962400 & -0.06601900 \\
\hline $\mathrm{H}$ & -4.51084900 & 1.29519200 & -0.84778100 \\
\hline $\mathrm{H}$ & -3.72966800 & 2.49201800 & 1.18752500 \\
\hline $\mathrm{H}$ & -4.12252800 & 1.10325500 & 2.18908200 \\
\hline $\mathrm{H}$ & -4.13351700 & -2.53637100 & -1.63265500 \\
\hline $\mathrm{O}$ & -6.06155600 & 1.45712100 & 0.50070700 \\
\hline $\mathrm{C}$ & -6.94797200 & 1.63272300 & -0.53470100 \\
\hline $\mathrm{O}$ & -6.67012800 & 1.36095900 & -1.67930200 \\
\hline $\mathrm{C}$ & -8.25612500 & 2.18221800 & -0.03431500 \\
\hline $\mathrm{H}$ & -8.74328800 & 1.44631700 & 0.61470200 \\
\hline $\mathrm{H}$ & -8.09123700 & 3.08440700 & 0.56255000 \\
\hline $\mathrm{H}$ & -8.90351400 & 2.40415000 & -0.88276500 \\
\hline $\mathrm{C}$ & 1.61198400 & -1.28866000 & 0.49318600 \\
\hline $\mathrm{H}$ & 1.89005900 & -0.85726600 & -0.47768500 \\
\hline $\mathrm{C}$ & 1.59868700 & -2.82574800 & 0.27788200 \\
\hline $\mathrm{C}$ & 0.44694200 & -3.14633600 & -0.70292700 \\
\hline $\mathrm{C}$ & 1.51618300 & -3.55224400 & 1.63198000 \\
\hline $\mathrm{H}$ & 1.47664800 & -4.63756700 & 1.51350800 \\
\hline $\mathrm{H}$ & 2.40142200 & -3.32790300 & 2.23583300 \\
\hline $\mathrm{H}$ & 0.63633400 & -3.25217900 & 2.2097070 \\
\hline
\end{tabular}




$\begin{array}{lrrr}\mathrm{C} & 2.78510000 & -3.35039900 & -0.56746600 \\ \mathrm{C} & 0.72857400 & -4.61791500 & -1.09598400 \\ \mathrm{C} & 2.28739100 & -4.71837500 & -1.08441900 \\ \mathrm{O} & 4.03400000 & -3.50847300 & 0.13512100 \\ \mathrm{C} & 4.84176600 & -2.43347900 & 0.26046200 \\ \mathrm{O} & 4.51593600 & -1.30033000 & -0.06125300 \\ \mathrm{C} & 6.18089600 & -2.82429200 & 0.82546100 \\ \mathrm{H} & 6.74245700 & -1.93253700 & 1.10522400 \\ \mathrm{H} & 6.05990000 & -3.48750300 & 1.68657000 \\ \mathrm{H} & 6.74435700 & -3.38048400 & 0.06752400 \\ \mathrm{C} & -2.52450100 & -1.34725200 & 2.22555200 \\ \mathrm{H} & -3.50311500 & -1.07947600 & 2.63061300 \\ \mathrm{H} & -2.47284500 & -2.43930900 & 2.21951000 \\ \mathrm{H} & -1.77602500 & -0.97113100 & 2.93194900 \\ \mathrm{H} & 0.29529400 & -4.85217600 & -2.06731700 \\ \mathrm{H} & -1.16328000 & -3.27841400 & 0.73711600 \\ \mathrm{H} & -0.91343900 & -0.66940100 & -0.82265200 \\ \mathrm{C} & -0.96246400 & -1.15483100 & 0.16600000 \\ \mathrm{C} & 0.26983200 & -0.78105100 & 0.96050800 \\ \mathrm{H} & 0.69855300 & 0.67318800 & 0.74577500 \\ \mathrm{H} & 0.14202000 & -0.80270000 & 2.04593300 \\ \mathrm{H} & 0.28248900 & -5.30992600 & -0.37440000 \\ \mathrm{H} & 1.55437400 & 1.11219800 & -1.49371600 \\ \mathrm{H} & 0.62955000 & -2.54448900 & -1.60892200 \\ \mathrm{H} & 2.63919700 & -5.50950400 & -0.41646600 \\ \mathrm{H} & 2.69590100 & -4.93300300 & -2.07504200 \\ \mathrm{H} & 2.96333400 & -2.65682600 & -1.39562300 \\ \mathrm{H} & 2.39674500 & -1.02861900 & 1.20665500\end{array}$

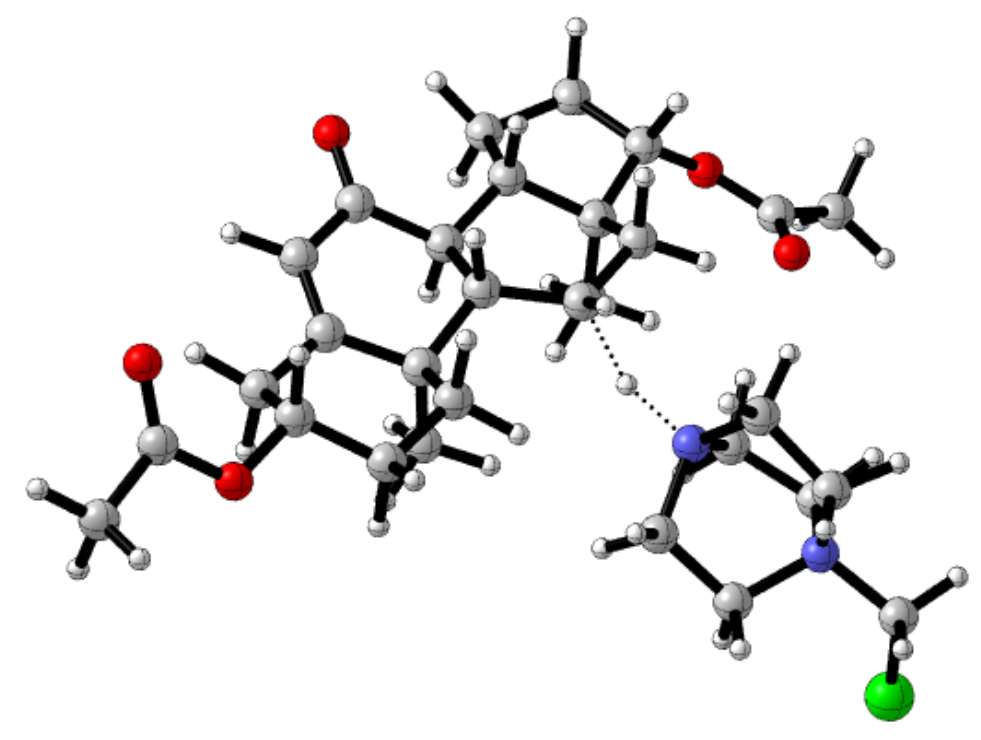

Figure S59. Transition state involving carbon C32 (top hydrogen) using SRD. 
Zero-point correction $=$

Thermal correction to Energy=

Thermal correction to Enthalpy=

Thermal correction to Gibbs Free Energy=

Sum of electronic and zero-point Energies=

Sum of electronic and thermal Energies=

Sum of electronic and thermal Enthalpies=

Sum of electronic and thermal Free Energies=
0.734459 (Hartree/Particle)

0.771936

0.772880

0.662631

$-2115.338893$

$-2115.301417$

$-2115.300473$

$-2115.410722$

Number of Imaginary Frequencies $=1$

E (Single Point Energy) [IEFPCM $\left(\mathrm{CH}_{3 \mathrm{CN})} \mathrm{U} \omega \mathrm{B} 97 \mathrm{X}-\mathrm{D} / 6-311++\mathrm{G}(2 \mathrm{~d}, 2 \mathrm{p})\right]=-2116.36850650$

$\begin{array}{lrrr}22 & & & \\ \mathrm{C} & 2.95586000 & -0.93915000 & -0.44823000 \\ \mathrm{C} & 2.15412500 & -1.74436600 & 1.70895400 \\ \mathrm{C} & 1.22080900 & -2.65368200 & -0.34956000 \\ \mathrm{C} & 4.11632200 & -1.94434100 & -0.25890700 \\ \mathrm{H} & 3.25525600 & 0.04057600 & -0.06683000 \\ \mathrm{C} & 3.07146900 & -2.99275500 & 1.71421000 \\ \mathrm{H} & 1.25402400 & -1.93346400 & 2.29738300 \\ \mathrm{H} & 2.66307600 & -0.87164600 & 2.12136600 \\ \mathrm{H} & 0.42360000 & -3.05490000 & 0.27970400 \\ \mathrm{H} & 0.78746900 & -2.37902500 & -1.31225800 \\ \mathrm{C} & 2.36863500 & -3.67631700 & -0.54752200 \\ \mathrm{H} & 4.85511300 & -1.58194600 & 0.45998900 \\ \mathrm{H} & 4.62047700 & -2.16827500 & -1.19977200 \\ \mathrm{H} & 2.54812100 & -3.89399100 & 2.04127100 \\ \mathrm{H} & 3.94439500 & -2.84266000 & 2.35319000 \\ \mathrm{H} & 2.07688100 & -4.68023300 & -0.23125500 \\ \mathrm{H} & 2.70849000 & -3.72581900 & -1.58192200 \\ \mathrm{~N} & 1.75614300 & -1.42418700 & 0.30478700 \\ \mathrm{~N} & 3.56314200 & -3.25140400 & 0.29601300 \\ \mathrm{C} & 4.63612600 & -4.33672800 & 0.35372600 \\ \mathrm{H} & 4.19649200 & -5.19838400 & 0.85781000 \\ \mathrm{H} & 5.46756500 & -3.93438900 & 0.93378100 \\ \mathrm{O} & -2.91821000 & 4.17826300 & -0.27234800 \\ \mathrm{Cl} & 5.21353500 & -4.82033100 & -1.24354800 \\ \mathrm{C} & -2.72366900 & 2.98089500 & -0.36958900 \\ \mathrm{C} & -3.82087500 & 2.01111200 & -0.50144800 \\ \mathrm{C} & -1.29155300 & 2.39627500 & -0.41617800 \\ \mathrm{C} & -3.65394000 & 0.67302700 & -0.49042000 \\ \mathrm{C} & -2.28594200 & 0.01343200 & -0.24916000 \\ \mathrm{C} & -4.82750000 & -0.24437400 & -0.73788600\end{array}$




\begin{tabular}{|c|c|c|c|}
\hline $\mathrm{C}$ & -2.46897400 & -1.17220100 & 0.74561600 \\
\hline $\mathrm{C}$ & -4.93631100 & -1.35815900 & 0.31543300 \\
\hline $\mathrm{H}$ & -4.71619700 & -0.72635500 & -1.72120200 \\
\hline $\mathrm{H}$ & -5.75488000 & 0.33202600 & -0.76352200 \\
\hline $\mathrm{C}$ & -3.62479400 & -2.12792100 & 0.41740800 \\
\hline $\mathrm{H}$ & -1.53073900 & -1.74497400 & 0.79872500 \\
\hline $\mathrm{H}$ & -2.63626500 & -0.76133300 & 1.75124500 \\
\hline $\mathrm{H}$ & -5.20303100 & -0.91436000 & 1.28066700 \\
\hline $\mathrm{H}$ & -3.70435600 & -2.89224600 & 1.19835000 \\
\hline $\mathrm{H}$ & -3.44821400 & -2.65892700 & -0.52650000 \\
\hline $\mathrm{H}$ & -4.80759900 & 2.44682900 & -0.63449400 \\
\hline $\mathrm{O}$ & -5.95471200 & -2.29927800 & -0.05213400 \\
\hline $\mathrm{C}$ & -7.24369000 & -1.94466900 & 0.26813900 \\
\hline $\mathrm{O}$ & -7.51321000 & -0.89306000 & 0.79838200 \\
\hline $\mathrm{C}$ & -8.21279300 & -3.02545900 & -0.12836600 \\
\hline $\mathrm{H}$ & -7.97105500 & -3.95845900 & 0.39122000 \\
\hline $\mathrm{H}$ & -9.22574700 & -2.71253900 & 0.12480700 \\
\hline $\mathrm{H}$ & -8.14132000 & -3.22161300 & -1.20316500 \\
\hline $\mathrm{C}$ & 1.08730100 & 1.61730700 & 1.22545100 \\
\hline $\mathrm{H}$ & 0.83424100 & 2.10810200 & 2.18389700 \\
\hline $\mathrm{C}$ & 1.16850900 & 2.69105000 & 0.13335800 \\
\hline $\mathrm{C}$ & -0.22720300 & 3.37587200 & 0.09194700 \\
\hline $\mathrm{C}$ & 1.55216800 & 2.11842000 & -1.24670900 \\
\hline $\mathrm{H}$ & 1.43907900 & 2.86545200 & -2.03657100 \\
\hline $\mathrm{H}$ & 2.59330100 & 1.79358800 & -1.26858300 \\
\hline $\mathrm{H}$ & 0.92149900 & 1.26449600 & -1.52094700 \\
\hline $\mathrm{C}$ & 2.08762300 & 3.89117000 & 0.49879900 \\
\hline $\mathrm{C}$ & 0.02102900 & 4.70256400 & -0.64480800 \\
\hline $\mathrm{C}$ & 1.41034700 & 5.14706600 & -0.11687900 \\
\hline $\mathrm{O}$ & 3.45660900 & 3.86544500 & -0.00857700 \\
\hline $\mathrm{C}$ & 4.34626700 & 2.91789300 & 0.30452700 \\
\hline $\mathrm{O}$ & 4.07160600 & 1.84755900 & 0.83929600 \\
\hline $\mathrm{C}$ & 5.73906700 & 3.33307900 & -0.09284800 \\
\hline $\mathrm{H}$ & 6.09627200 & 4.09937500 & 0.60500100 \\
\hline $\mathrm{H}$ & 6.41279700 & 2.47650200 & -0.05324000 \\
\hline $\mathrm{H}$ & 5.74206900 & 3.78115100 & -1.09014600 \\
\hline $\mathrm{C}$ & -1.74371000 & -0.49726100 & -1.60695000 \\
\hline $\mathrm{H}$ & -2.36389500 & -1.30341000 & -2.00674000 \\
\hline $\mathrm{H}$ & -1.71600000 & 0.29238500 & -2.36250100 \\
\hline $\mathrm{H}$ & -0.72370200 & -0.88646000 & -1.49382200 \\
\hline $\mathrm{H}$ & -0.76188600 & 5.43115500 & -0.43804100 \\
\hline $\mathrm{H}$ & -1.11287900 & 2.18818100 & -1.47985700 \\
\hline $\mathrm{H}$ & -1.83260500 & 1.33473400 & 1.34061900 \\
\hline $\mathrm{C}$ & -1.32442200 & 1.07151200 & 0.38663500 \\
\hline $\mathrm{C}$ & 0.00605900 & 0.58772400 & 0.97531300 \\
\hline $\mathrm{H}$ & 0.82468500 & -0.47696500 & 0.37518400 \\
\hline
\end{tabular}




$\begin{array}{rrrr}\mathrm{H} & -0.16919200 & -0.05210500 & 1.85171300 \\ \mathrm{H} & 0.03863800 & 4.55516000 & -1.73087400 \\ \mathrm{H} & 2.67081900 & -0.83948100 & -1.49694700 \\ \mathrm{H} & -0.49228100 & 3.63644800 & 1.12871200 \\ \mathrm{H} & 2.04538700 & 5.55340600 & -0.90807600 \\ \mathrm{H} & 1.31328300 & 5.92777100 & 0.64241000 \\ \mathrm{H} & 2.17102300 & 3.97215100 & 1.58790400 \\ \mathrm{H} & 2.06800600 & 1.17172200 & 1.41576500\end{array}$

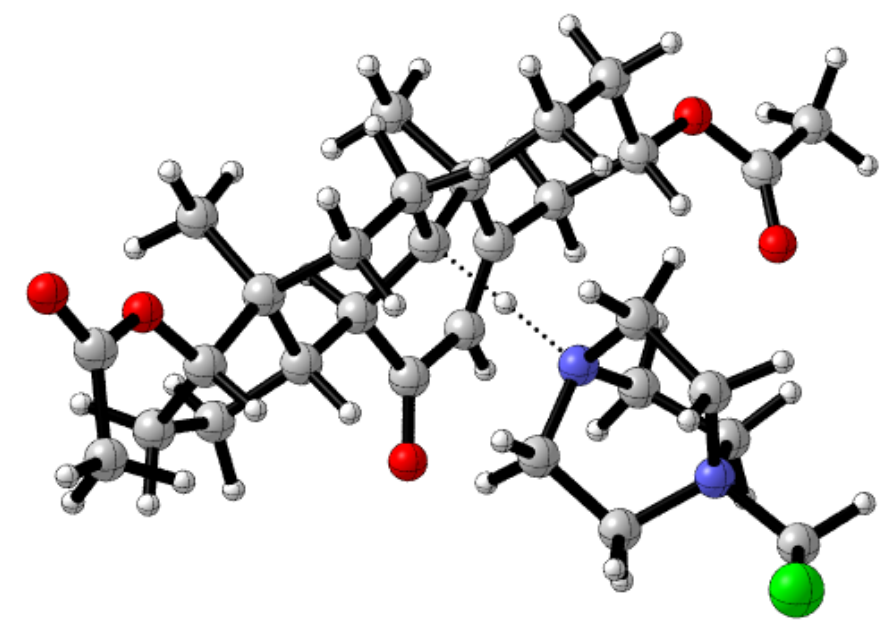

Figure S60. Transition state involving carbon C28 using SRD.

Zero-point correction $=$

Thermal correction to Energy=

Thermal correction to Enthalpy=

Thermal correction to Gibbs Free Energy=

Sum of electronic and zero-point Energies=

Sum of electronic and thermal Energies=

Sum of electronic and thermal Enthalpies=

Sum of electronic and thermal Free Energies=
0.733886 (Hartree/Particle)

0.771583

0.772528

0.661031

$-2115.337440$

$-2115.299743$

$-2115.298798$

$-2115.410295$

Number of Imaginary Frequencies $=1$

E (Single Point Energy) [IEFPCM $\left(\mathrm{CH}_{3 \mathrm{CN}} \mathrm{U} \omega \mathrm{BB} 97 \mathrm{X}-\mathrm{D} / 6-311++\mathrm{G}(2 \mathrm{~d}, 2 \mathrm{p})\right]=-2116.37241681$

22

$\begin{array}{lrrr}\mathrm{C} & -0.40092100 & 2.11300900 & 0.63616800 \\ \mathrm{C} & 1.92578200 & 1.40657000 & 0.57132500 \\ \mathrm{C} & 0.73866700 & 1.90085900 & -1.50441800 \\ \mathrm{C} & 0.17006200 & 3.51963600 & 0.93814600 \\ \mathrm{H} & -0.62387100 & 1.58621800 & 1.56552000\end{array}$




\begin{tabular}{|c|c|c|c|}
\hline $\mathrm{C}$ & 2.48710100 & 2.84424600 & 0.44238500 \\
\hline $\mathrm{H}$ & 2.62191300 & 0.69245400 & 0.13148300 \\
\hline $\mathrm{H}$ & 1.76118100 & 1.12733100 & 1.61245800 \\
\hline $\mathrm{H}$ & 1.57136600 & 1.40726300 & -2.00827600 \\
\hline $\mathrm{H}$ & -0.17521300 & 1.68980800 & -2.06270800 \\
\hline $\mathrm{C}$ & 0.96358300 & 3.43216900 & -1.40389800 \\
\hline $\mathrm{H}$ & 0.53604100 & 3.60543300 & 1.96395700 \\
\hline $\mathrm{H}$ & -0.56766300 & 4.30347000 & 0.76147500 \\
\hline $\mathrm{H}$ & 3.26787400 & 2.92129500 & -0.31758600 \\
\hline $\mathrm{H}$ & 2.88970300 & 3.19903300 & 1.39373200 \\
\hline $\mathrm{H}$ & 1.76643200 & 3.76632000 & -2.06473300 \\
\hline $\mathrm{H}$ & 0.06499700 & 4.00336200 & -1.63694200 \\
\hline $\mathrm{N}$ & 0.61116700 & 1.32230000 & -0.13503700 \\
\hline $\mathrm{N}$ & 1.35953800 & 3.78015700 & 0.02421100 \\
\hline $\mathrm{C}$ & 1.85909100 & 5.21787700 & 0.14508500 \\
\hline $\mathrm{H}$ & 2.77120300 & 5.29286900 & -0.44840500 \\
\hline $\mathrm{H}$ & 2.07119500 & 5.39594300 & 1.20005100 \\
\hline $\mathrm{O}$ & -0.31453500 & -0.22744000 & 2.88329000 \\
\hline $\mathrm{Cl}$ & 0.68346700 & 6.40539200 & -0.42717800 \\
\hline $\mathrm{C}$ & -0.00050900 & -1.06552000 & 2.04944800 \\
\hline $\mathrm{C}$ & 1.36009000 & -1.60470300 & 1.94262100 \\
\hline $\mathrm{C}$ & -0.98800800 & -1.54749300 & 0.96204600 \\
\hline $\mathrm{C}$ & 1.85619100 & -2.11619900 & 0.79433300 \\
\hline $\mathrm{C}$ & 1.01363900 & -2.23658600 & -0.48687900 \\
\hline $\mathrm{C}$ & 3.29205500 & -2.57683900 & 0.72435100 \\
\hline $\mathrm{C}$ & 1.84631200 & -1.85054500 & -1.75105400 \\
\hline $\mathrm{C}$ & 4.00139200 & -1.99619100 & -0.50981900 \\
\hline $\mathrm{H}$ & 3.33339200 & -3.67317700 & 0.65746900 \\
\hline $\mathrm{H}$ & 3.82311700 & -2.29206900 & 1.63696000 \\
\hline $\mathrm{C}$ & 3.27786600 & -2.40933400 & -1.78267500 \\
\hline $\mathrm{H}$ & 1.30497500 & -2.17392200 & -2.64561800 \\
\hline $\mathrm{H}$ & 1.91439500 & -0.75430300 & -1.81549400 \\
\hline $\mathrm{H}$ & 4.01993900 & -0.90242900 & -0.43075900 \\
\hline $\mathrm{H}$ & 3.80219600 & -2.01659900 & -2.66022100 \\
\hline $\mathrm{H}$ & 3.28112600 & -3.50059100 & -1.88059700 \\
\hline $\mathrm{H}$ & 1.98683200 & -1.49674800 & 2.82492900 \\
\hline $\mathrm{O}$ & 5.35794100 & -2.44068000 & -0.54840300 \\
\hline $\mathrm{C}$ & 6.28438300 & -1.59054800 & 0.00536500 \\
\hline $\mathrm{O}$ & 5.97505100 & -0.52270100 & 0.48981800 \\
\hline $\mathrm{C}$ & 7.66710400 & -2.16977200 & -0.08216600 \\
\hline $\mathrm{H}$ & 8.38840300 & -1.45563300 & 0.31493700 \\
\hline $\mathrm{H}$ & 7.71693100 & -3.10241500 & 0.48995400 \\
\hline $\mathrm{H}$ & 7.91081200 & -2.41466800 & -1.12077200 \\
\hline $\mathrm{C}$ & -2.54158400 & -0.64788200 & -1.44637400 \\
\hline $\mathrm{H}$ & -2.49073700 & 0.44663800 & -1.32563300 \\
\hline 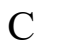 & -3.23165500 & -1.26099500 & -0.2210110 \\
\hline
\end{tabular}




$\begin{array}{lrrr}\mathrm{C} & -2.38406900 & -0.93008100 & 1.02783700 \\ \mathrm{C} & -3.46184700 & -2.77492400 & -0.42893000 \\ \mathrm{H} & -3.96881500 & -3.23374200 & 0.42345700 \\ \mathrm{H} & -4.10040700 & -2.93429200 & -1.30089200 \\ \mathrm{H} & -2.52945700 & -3.32746100 & -0.58532900 \\ \mathrm{C} & -4.56709800 & -0.59071800 & 0.18856300 \\ \mathrm{C} & -3.30069700 & -1.28448600 & 2.21869000 \\ \mathrm{C} & -4.73203600 & -0.95622200 & 1.69255500 \\ \mathrm{O} & -5.59357000 & -1.06100000 & -0.67529700 \\ \mathrm{C} & -6.82597800 & -0.45472300 & -0.83207200 \\ \mathrm{O} & -7.56758600 & -0.92087500 & -1.65247900 \\ \mathrm{C} & -7.15615400 & 0.73741500 & 0.03962500 \\ \mathrm{H} & -8.13192000 & 1.11508200 & -0.26607000 \\ \mathrm{H} & -7.20171500 & 0.45012600 & 1.09536900 \\ \mathrm{H} & -6.41591700 & 1.53912500 & -0.06039800 \\ \mathrm{C} & 0.61374200 & -3.74979100 & -0.62280500 \\ \mathrm{H} & 1.51207600 & -4.36053000 & -0.73580300 \\ \mathrm{H} & 0.07757900 & -4.11604800 & 0.25576500 \\ \mathrm{H} & -0.00560500 & -3.91137400 & -1.50957600 \\ \mathrm{H} & -3.03912600 & -0.71968700 & 3.11570600 \\ \mathrm{H} & -1.06671500 & -2.63232800 & 1.14693200 \\ \mathrm{H} & 0.19914200 & -0.02804300 & -0.25439200 \\ \mathrm{C} & -0.27011900 & -1.37728500 & -0.37389100 \\ \mathrm{C} & -1.12676300 & -1.22976300 & -1.63776200 \\ \mathrm{H} & -1.22292800 & -2.24806700 & -2.04670000 \\ \mathrm{H} & -3.21309900 & -2.34633200 & 2.47371900 \\ \mathrm{H} & -1.30930900 & 2.16969700 & 0.03420600 \\ \mathrm{H} & -2.26767100 & 0.16353500 & 1.03863000 \\ \mathrm{H} & -5.39954800 & -1.81776300 & 1.78262400 \\ \mathrm{H} & -5.18794200 & -0.13706200 & 2.25436300 \\ \mathrm{H} & -4.46266200 & 0.49843100 & 0.07585800 \\ \mathrm{H} & -3.12015600 & -0.82699600 & -2.35945200 \\ \mathrm{H} & -0.58234800 & -0.68552700 & -2.41953400\end{array}$




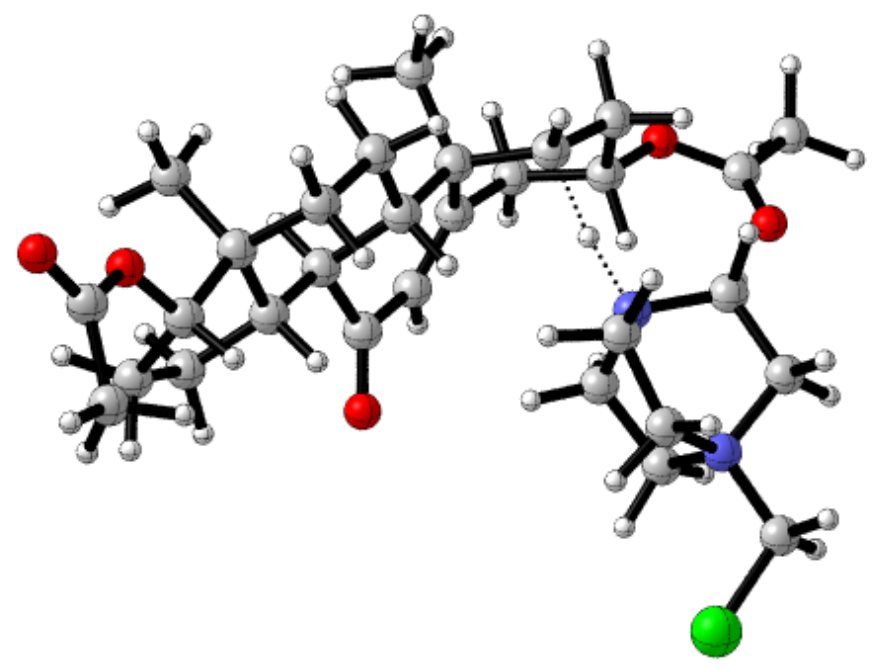

Figure S61. Transition state involving carbon C5 (bottom hydrogen) using SRD.

Zero-point correction $=$

Thermal correction to Energy=

Thermal correction to Enthalpy=

Thermal correction to Gibbs Free Energy=

Sum of electronic and zero-point Energies $=$

Sum of electronic and thermal Energies=

Sum of electronic and thermal Enthalpies=

Sum of electronic and thermal Free Energies=
0.734638 (Hartree/Particle)

0.772116

0.773061

0.662609

$-2115.333464$

$-2115.295985$

$-2115.295041$

$-2115.405493$

Number of Imaginary Frequencies $=1$

E (Single Point Energy) IEFPCM $_{(\mathrm{CH} 3 \mathrm{CN})} \mathrm{U \omega B}$ B7X-D/6-311++G(2d,2p)] $=-2116.37265293$

22

$\begin{array}{lrrr}\mathrm{C} & 2.06324600 & 1.16285400 & 0.74093900 \\ \mathrm{C} & 3.42915500 & 1.02648500 & -1.29908100 \\ \mathrm{C} & 1.13719000 & 1.84971200 & -1.41013600 \\ \mathrm{C} & 2.75750600 & 2.52167700 & 0.99686400 \\ \mathrm{H} & 2.60137000 & 0.35988500 & 1.24368600 \\ \mathrm{C} & 3.87043100 & 2.50958500 & -1.20652600 \\ \mathrm{H} & 3.39723800 & 0.70905600 & -2.34362200 \\ \mathrm{H} & 4.11242000 & 0.36016100 & -0.76273300 \\ \mathrm{H} & 1.21618200 & 1.68834000 & -2.48740200 \\ \mathrm{H} & 0.11141200 & 1.63826100 & -1.10349700 \\ \mathrm{C} & 1.53488200 & 3.29620400 & -1.01658000 \\ \mathrm{H} & 3.76910900 & 2.40199600 & 1.39136800 \\ \mathrm{H} & 2.18288800 & 3.14594000 & 1.68218300 \\ \mathrm{H} & 3.87480300 & 3.00607000 & -2.17944500\end{array}$




\begin{tabular}{|c|c|c|c|}
\hline $\mathrm{H}$ & 4.86371300 & 2.59959800 & -0.76138600 \\
\hline $\mathrm{H}$ & 1.62201300 & 3.94521000 & -1.89063600 \\
\hline $\mathrm{H}$ & 0.82682100 & 3.74576000 & -0.32044500 \\
\hline $\mathrm{N}$ & 2.05231200 & 0.88724300 & -0.73000100 \\
\hline $\mathrm{N}$ & 2.89021500 & 3.27216900 & -0.32199500 \\
\hline $\mathrm{C}$ & 3.44255800 & 4.68068800 & -0.10895400 \\
\hline $\mathrm{H}$ & 3.60073700 & 5.11674600 & -1.09606200 \\
\hline $\mathrm{H}$ & 4.38990600 & 4.57595800 & 0.42117900 \\
\hline $\mathrm{O}$ & -0.78447900 & 0.06934200 & 2.73617100 \\
\hline $\mathrm{Cl}$ & 2.35769100 & 5.71511200 & 0.82499800 \\
\hline $\mathrm{C}$ & -0.70881800 & -0.95342900 & 2.06729000 \\
\hline $\mathrm{C}$ & 0.50421000 & -1.80204500 & 2.11701500 \\
\hline $\mathrm{C}$ & -1.73633600 & -1.37154600 & 1.02912200 \\
\hline $\mathrm{C}$ & 0.97928300 & -2.40896900 & 1.01567900 \\
\hline $\mathrm{C}$ & 0.29823400 & -2.26479400 & -0.35737700 \\
\hline $\mathrm{C}$ & 2.30284100 & -3.15166500 & 1.04617700 \\
\hline $\mathrm{C}$ & 1.34255400 & -1.72353700 & -1.33358000 \\
\hline $\mathrm{C}$ & 3.27895100 & -2.43966100 & 0.10695100 \\
\hline $\mathrm{H}$ & 2.19097600 & -4.19649400 & 0.73391400 \\
\hline $\mathrm{H}$ & 2.70759600 & -3.16494900 & 2.06223300 \\
\hline $\mathrm{C}$ & 2.71788200 & -2.35262300 & -1.31857600 \\
\hline $\mathrm{H}$ & 0.95042600 & -1.50626300 & -2.33430700 \\
\hline $\mathrm{H}$ & 1.64866500 & -0.34306700 & -0.95838000 \\
\hline $\mathrm{H}$ & 3.45174100 & -1.43006300 & 0.49024500 \\
\hline $\mathrm{H}$ & 3.41412900 & -1.82383500 & -1.97838800 \\
\hline $\mathrm{H}$ & 2.64079600 & -3.37301800 & -1.73107500 \\
\hline $\mathrm{H}$ & 1.04080700 & -1.84022700 & 3.06284200 \\
\hline $\mathrm{O}$ & 4.54528500 & -3.10960400 & 0.11101700 \\
\hline $\mathrm{C}$ & 5.64046600 & -2.32574300 & -0.08649300 \\
\hline $\mathrm{O}$ & 5.55862700 & -1.12408700 & -0.28292100 \\
\hline $\mathrm{C}$ & 6.91013200 & -3.12173700 & -0.02321300 \\
\hline $\mathrm{H}$ & 6.88533900 & -3.92764400 & -0.76395000 \\
\hline $\mathrm{H}$ & 7.76381200 & -2.47010700 & -0.20844000 \\
\hline $\mathrm{H}$ & 7.00614900 & -3.59263500 & 0.96088300 \\
\hline $\mathrm{C}$ & -3.13520900 & -0.54696000 & -1.50185800 \\
\hline $\mathrm{H}$ & -2.82394400 & 0.50789700 & -1.56770200 \\
\hline $\mathrm{C}$ & -3.90925700 & -0.76604500 & -0.19333100 \\
\hline $\mathrm{C}$ & -2.96388600 & -0.45960500 & 0.99129200 \\
\hline $\mathrm{C}$ & -4.51612900 & -2.18754200 & -0.14617500 \\
\hline $\mathrm{H}$ & -5.11111300 & -2.35121200 & 0.75586600 \\
\hline $\mathrm{H}$ & -5.18316500 & -2.33550300 & -0.99902000 \\
\hline $\mathrm{H}$ & -3.75333100 & -2.97099400 & -0.17524700 \\
\hline $\mathrm{C}$ & -5.02053700 & 0.27134900 & 0.10198700 \\
\hline $\mathrm{C}$ & -3.89674200 & -0.39140400 & 2.21839000 \\
\hline $\mathrm{C}$ & -5.21601000 & 0.20824300 & 1.64355100 \\
\hline $\mathrm{O}$ & -6.18638400 & -0.06679300 & -0.6596840 \\
\hline
\end{tabular}




$\begin{array}{lrrr}\mathrm{C} & -7.19712000 & 0.80426800 & -0.93352200 \\ \mathrm{O} & -8.12449000 & 0.40116200 & -1.59373200 \\ \mathrm{C} & -7.11069600 & 2.22523200 & -0.41595300 \\ \mathrm{H} & -8.01543400 & 2.75170500 & -0.71941000 \\ \mathrm{H} & -7.02944600 & 2.24503000 & 0.67523100 \\ \mathrm{H} & -6.24005700 & 2.74336300 & -0.83374700 \\ \mathrm{C} & -0.10946400 & -3.69561900 & -0.86045200 \\ \mathrm{H} & 0.71405800 & -4.40512300 & -0.76895200 \\ \mathrm{H} & -0.94196400 & -4.06788500 & -0.25476800 \\ \mathrm{H} & -0.42146100 & -3.68139100 & -1.90688900 \\ \mathrm{H} & -3.47002000 & 0.21769900 & 3.01728400 \\ \mathrm{H} & -2.02896100 & -2.40938400 & 1.23676200 \\ \mathrm{C} & -0.96292200 & -1.31276800 & -0.33185300 \\ \mathrm{C} & -1.89547400 & -1.46297900 & -1.55943300 \\ \mathrm{H} & -2.23550500 & -2.49725000 & -1.64443300 \\ \mathrm{H} & -4.07170200 & -1.39013200 & 2.63292600 \\ \mathrm{H} & 1.03444500 & 1.15367900 & 1.10735400 \\ \mathrm{H} & -2.58090600 & 0.56255600 & 0.83156500 \\ \mathrm{H} & -6.07999100 & -0.42206700 & 1.87263800 \\ \mathrm{H} & -5.42162600 & 1.19826100 & 2.05992900 \\ \mathrm{H} & -4.66869500 & 1.26578800 & -0.20099100 \\ \mathrm{H} & -3.76808700 & -0.74313900 & -2.37465300 \\ \mathrm{H} & -1.33162600 & -1.25495200 & -2.47945800 \\ \mathrm{H} & -0.57294900 & -0.28032700 & -0.37382500\end{array}$

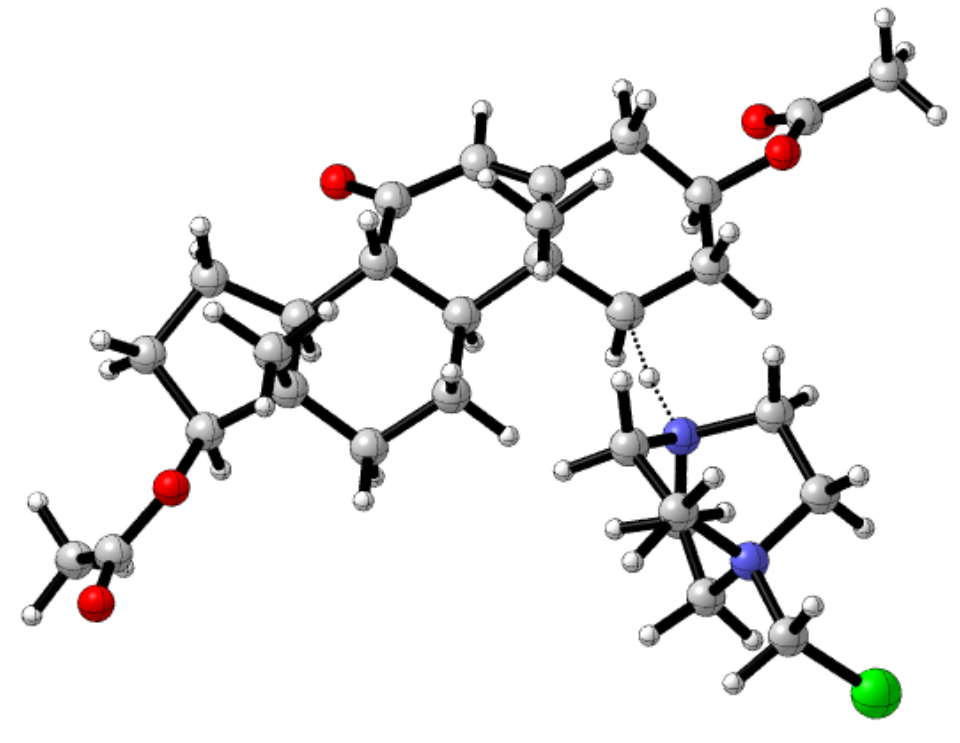

Figure S62. Transition state involving carbon C5 (top hydrogen) using SRD.

Zero-point correction=

Thermal correction to Energy=

Thermal correction to Enthalpy=
0.733692 (Hartree/Particle)

0.771662

0.772606 


$\begin{array}{ll}\text { Thermal correction to Gibbs Free Energy= } & 0.659037 \\ \text { Sum of electronic and zero-point Energies= } & -2115.318875 \\ \text { Sum of electronic and thermal Energies= } & -2115.280905 \\ \text { Sum of electronic and thermal Enthalpies= } & -2115.279961 \\ \text { Sum of electronic and thermal Free Energies= } & -2115.393529\end{array}$

Number of Imaginary Frequencies $=1$

E (Single Point Energy) [IEFPCM $\left.{ }_{(\mathrm{CH} 3 \mathrm{CN})} \mathrm{U} \omega \mathrm{B} 97 \mathrm{X}-\mathrm{D} / 6-311++\mathrm{G}(2 \mathrm{~d}, 2 \mathrm{p})\right]=-2116.37011876$

$\begin{array}{lrrr}2 \mathrm{C} & & & \\ \mathrm{C} & -3.77256500 & 1.40295600 & 0.52172700 \\ \mathrm{C} & -1.59860100 & 2.27843100 & 1.18967000 \\ \mathrm{C} & -2.19098300 & 2.14826400 & -1.18230800 \\ \mathrm{C} & -4.41778700 & 2.80356200 & 0.37359600 \\ \mathrm{H} & -3.83778200 & 1.02998900 & 1.54584500 \\ \mathrm{C} & -2.33579600 & 3.62265300 & 1.41050500 \\ \mathrm{H} & -0.57472900 & 2.42644700 & 0.84697200 \\ \mathrm{H} & -1.56870600 & 1.69886200 & 2.11407100 \\ \mathrm{H} & -1.16033100 & 2.03995300 & -1.52557200 \\ \mathrm{H} & -2.84381300 & 1.62498200 & -1.88437400 \\ \mathrm{C} & -2.56826800 & 3.64188500 & -1.04805700 \\ \mathrm{H} & -5.03455100 & 3.05801900 & 1.23827600 \\ \mathrm{H} & -5.03115200 & 2.88836200 & -0.52352300 \\ \mathrm{H} & -1.63668800 & 4.46169900 & 1.42954700 \\ \mathrm{H} & -2.91803300 & 3.63216200 & 2.33458300 \\ \mathrm{H} & -1.69048500 & 4.29158800 & -1.01705200 \\ \mathrm{H} & -3.21862900 & 3.97099200 & -1.85953800 \\ \mathrm{~N} & -2.32429300 & 1.48169700 & 0.15170400 \\ \mathrm{~N} & -3.31457600 & 3.84857900 & 0.26415100 \\ \mathrm{C} & -3.86459000 & 5.27240800 & 0.37614500 \\ \mathrm{H} & -3.02169200 & 5.95212600 & 0.24411200 \\ \mathrm{H} & -4.28769900 & 5.37415700 & 1.37640500 \\ \mathrm{O} & 3.08123600 & -4.03169900 & -0.40819600 \\ \mathrm{Cl} & -5.10339000 & 5.62663100 & -0.82961900 \\ \mathrm{C} & 2.16063900 & -3.34046500 & -0.01430600 \\ \mathrm{C} & 0.79233900 & -3.90293300 & 0.08534500 \\ \mathrm{C} & 2.32616500 & -1.88882000 & 0.43606000 \\ \mathrm{C} & -0.31924900 & -3.19976400 & 0.37785100 \\ \mathrm{C} & -0.26887200 & -1.65111800 & 0.56503300 \\ \mathrm{C} & -1.64101200 & -3.89926600 & 0.56925700 \\ \mathrm{C} & -1.46551000 & -1.14290200 & -0.25606900 \\ \mathrm{C} & -2.72439200 & -3.30153000 & -0.33166200 \\ \mathrm{H} & -1.97933300 & -3.82197000 & 1.61223400 \\ \mathrm{H} & -1.53529600 & -4.96279200 & 0.33814900 \\ \mathrm{C} & -2.81918300 & -1.77440600 & -0.05109700 \\ \mathrm{H} & -1.21813000 & -1.00887400 & -1.31704600\end{array}$




\begin{tabular}{|c|c|c|c|}
\hline $\mathrm{H}$ & -1.84274700 & 0.31372700 & 0.05207500 \\
\hline $\mathrm{H}$ & -2.47519800 & -3.48271600 & -1.38156300 \\
\hline $\mathrm{H}$ & -3.56825300 & -1.36127800 & -0.73720100 \\
\hline $\mathrm{H}$ & -3.18897700 & -1.66222200 & 0.97578400 \\
\hline $\mathrm{H}$ & 0.74029100 & -4.98354800 & -0.02706500 \\
\hline $\mathrm{O}$ & -4.01052300 & -3.83926700 & -0.05149200 \\
\hline $\mathrm{C}$ & -4.34390200 & -4.99640400 & -0.74583500 \\
\hline $\mathrm{O}$ & -3.58466600 & -5.50871800 & -1.52744400 \\
\hline $\mathrm{C}$ & -5.71554300 & -5.47328500 & -0.36012000 \\
\hline $\mathrm{H}$ & -6.44881900 & -4.66874700 & -0.47110900 \\
\hline $\mathrm{H}$ & -5.98966900 & -6.32196900 & -0.98681400 \\
\hline $\mathrm{H}$ & -5.71895700 & -5.77982200 & 0.69160500 \\
\hline $\mathrm{C}$ & 2.55274000 & 0.97809500 & -0.44528900 \\
\hline $\mathrm{H}$ & 2.60261900 & 0.83047600 & -1.53330900 \\
\hline $\mathrm{C}$ & 3.72709900 & 0.25230000 & 0.22255600 \\
\hline $\mathrm{C}$ & 3.62123300 & -1.25750100 & -0.09601500 \\
\hline $\mathrm{C}$ & 3.77088800 & 0.56265400 & 1.73642300 \\
\hline $\mathrm{H}$ & 4.62665300 & 0.09211700 & 2.22592700 \\
\hline $\mathrm{H}$ & 3.87051500 & 1.64030000 & 1.89162600 \\
\hline $\mathrm{H}$ & 2.87495300 & 0.21906500 & 2.26398700 \\
\hline $\mathrm{C}$ & 5.11080800 & 0.55009400 & -0.39830800 \\
\hline $\mathrm{C}$ & 4.99465400 & -1.81962100 & 0.33782600 \\
\hline $\mathrm{C}$ & 5.98899500 & -0.65521000 & 0.03625800 \\
\hline $\mathrm{O}$ & 5.54392300 & 1.83519500 & 0.05693300 \\
\hline $\mathrm{C}$ & 6.51491200 & 2.58720000 & -0.55756700 \\
\hline $\mathrm{O}$ & 6.68546400 & 3.70896100 & -0.15370300 \\
\hline $\mathrm{C}$ & 7.30953000 & 1.96061900 & -1.68237200 \\
\hline $\mathrm{H}$ & 8.01356300 & 2.70519800 & -2.05391400 \\
\hline $\mathrm{H}$ & 7.86540600 & 1.08624200 & -1.32873500 \\
\hline $\mathrm{H}$ & 6.66552100 & 1.63555300 & -2.50702100 \\
\hline $\mathrm{C}$ & -0.42077000 & -1.35171300 & 2.07622000 \\
\hline $\mathrm{H}$ & -1.34915000 & -1.76509700 & 2.48026100 \\
\hline $\mathrm{H}$ & 0.40046100 & -1.79473800 & 2.64543300 \\
\hline $\mathrm{H}$ & -0.41446300 & -0.27540400 & 2.28121000 \\
\hline $\mathrm{H}$ & 5.24020500 & -2.73443200 & -0.20041800 \\
\hline $\mathrm{H}$ & 2.35174800 & -1.90276100 & 1.53612200 \\
\hline $\mathrm{C}$ & 1.06638300 & -1.10485300 & -0.05093900 \\
\hline $\mathrm{C}$ & 1.21682200 & 0.42728500 & 0.08473900 \\
\hline $\mathrm{H}$ & 1.10249800 & 0.72753500 & 1.13254200 \\
\hline $\mathrm{H}$ & 4.99538200 & -2.06881700 & 1.40475600 \\
\hline $\mathrm{H}$ & -4.26497300 & 0.68481800 & -0.13477200 \\
\hline $\mathrm{H}$ & 3.57475000 & -1.34484000 & -1.19371400 \\
\hline $\mathrm{H}$ & 6.57218000 & -0.37975200 & 0.92009100 \\
\hline $\mathrm{H}$ & 6.70129800 & -0.93038300 & -0.74576500 \\
\hline $\mathrm{H}$ & 5.00846600 & 0.57011600 & -1.49109600 \\
\hline $\mathrm{H}$ & 2.61105100 & 2.05927000 & -0.26545100 \\
\hline
\end{tabular}




$$
\begin{array}{lrrr}
\mathrm{H} & 0.39120800 & 0.89436800 & -0.47768900 \\
\mathrm{H} & 0.99861900 & -1.31010000 & -1.13072000
\end{array}
$$

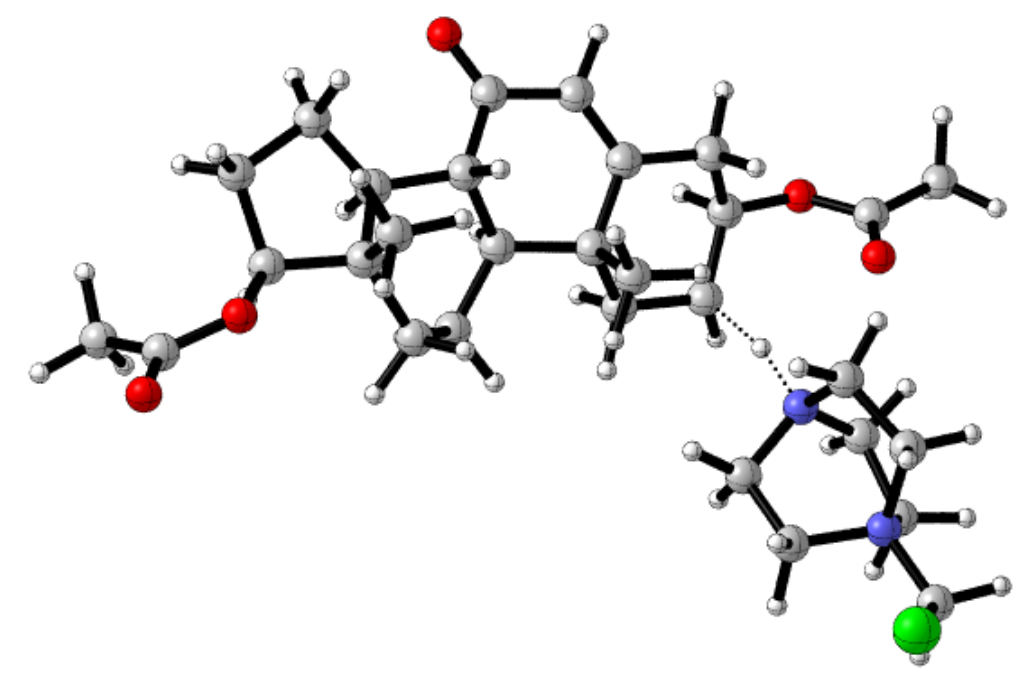

Figure S63. Transition state involving carbon C1 (top hydrogen) using SRD.

Zero-point correction=

Thermal correction to Energy=

Thermal correction to Enthalpy=

Thermal correction to Gibbs Free Energy=

Sum of electronic and zero-point Energies=

Sum of electronic and thermal Energies=

Sum of electronic and thermal Enthalpies=

Sum of electronic and thermal Free Energies=
0.734525 (Hartree/Particle)

0.771863

0.772808

0.662907

$-2115.312250$

$-2115.274911$

$-2115.273967$

$-2115.383868$

Number of Imaginary Frequencies $=1$

E (Single Point Energy) [IEFPCM $(\mathrm{CH} 3 \mathrm{CN}) \mathrm{U} \omega \mathrm{B} 97 \mathrm{X}-\mathrm{D} / 6-311++\mathrm{G}(2 \mathrm{~d}, 2 \mathrm{p})]=-2116.35370719$

22

$\begin{array}{lrrr}\mathrm{C} & 2.90049700 & 1.57037100 & 1.05137300 \\ \mathrm{C} & 4.86578500 & 0.12865900 & 1.10195300 \\ \mathrm{C} & 3.74871400 & 0.60504200 & -1.01902800 \\ \mathrm{C} & 3.69792000 & 2.83666400 & 0.64982700 \\ \mathrm{H} & 2.87529500 & 1.43465200 & 2.13498800 \\ \mathrm{C} & 5.67885600 & 1.44512000 & 1.10864200 \\ \mathrm{H} & 5.35986300 & -0.66679000 & 0.54556800 \\ \mathrm{H} & 4.69114000 & -0.21655600 & 2.12350800 \\ \mathrm{H} & 4.02958500 & -0.34279000 & -1.47593700 \\ \mathrm{H} & 2.79790000 & 0.93854200 & -1.43700400\end{array}$




\begin{tabular}{|c|c|c|c|}
\hline $\mathrm{C}$ & 4.85517200 & 1.66753700 & -1.20674800 \\
\hline $\mathrm{H}$ & 3.86945600 & 3.49679900 & 1.50285300 \\
\hline $\mathrm{H}$ & 3.20444300 & 3.40924900 & -0.13542000 \\
\hline $\mathrm{H}$ & 6.71612700 & 1.27344700 & 0.81247800 \\
\hline $\mathrm{H}$ & 5.67061600 & 1.94274700 & 2.08107500 \\
\hline $\mathrm{H}$ & 5.81948900 & 1.22364700 & -1.46493300 \\
\hline $\mathrm{H}$ & 4.59039600 & 2.40077600 & -1.96963200 \\
\hline $\mathrm{N}$ & 3.54348200 & 0.36708700 & 0.44502100 \\
\hline $\mathrm{N}$ & 5.05725700 & 2.41323500 & 0.10852400 \\
\hline $\mathrm{C}$ & 6.00066200 & 3.60133400 & -0.05819300 \\
\hline $\mathrm{H}$ & 6.92495400 & 3.21851900 & -0.49265700 \\
\hline $\mathrm{H}$ & 6.18373800 & 4.01000300 & 0.93642800 \\
\hline $\mathrm{O}$ & -3.63395800 & -3.78516400 & -0.76638300 \\
\hline $\mathrm{Cl}$ & 5.34058300 & 4.86456100 & -1.10154900 \\
\hline $\mathrm{C}$ & -2.71377800 & -2.99849500 & -0.65137500 \\
\hline $\mathrm{C}$ & -1.29697500 & -3.44841500 & -0.74571100 \\
\hline $\mathrm{C}$ & -2.86564200 & -1.49663700 & -0.46052300 \\
\hline $\mathrm{C}$ & -0.23996500 & -2.67740300 & -0.42988800 \\
\hline $\mathrm{C}$ & -0.38133500 & -1.27056500 & 0.17479100 \\
\hline $\mathrm{C}$ & 1.16815200 & -3.20414700 & -0.62250900 \\
\hline $\mathrm{C}$ & 0.55681600 & -1.16688800 & 1.42339900 \\
\hline $\mathrm{C}$ & 1.93840200 & -3.13089300 & 0.70310400 \\
\hline $\mathrm{H}$ & 1.70641500 & -2.62552700 & -1.38368800 \\
\hline $\mathrm{H}$ & 1.13905200 & -4.24276600 & -0.96261800 \\
\hline $\mathrm{C}$ & 1.95854400 & -1.74112100 & 1.29452100 \\
\hline $\mathrm{H}$ & 0.07747500 & -1.72778500 & 2.24657600 \\
\hline $\mathrm{H}$ & 1.38529000 & -3.74357500 & 1.43184100 \\
\hline $\mathrm{H}$ & 2.82102600 & -0.66520000 & 0.64823200 \\
\hline $\mathrm{H}$ & 2.54117100 & -1.68305500 & 2.22491800 \\
\hline $\mathrm{H}$ & -1.15942700 & -4.46084800 & -1.11893200 \\
\hline $\mathrm{O}$ & 3.19506000 & -3.84710000 & 0.65370200 \\
\hline $\mathrm{C}$ & 4.23418200 & -3.38611100 & -0.04913000 \\
\hline $\mathrm{O}$ & 4.26091800 & -2.26197100 & -0.53855300 \\
\hline $\mathrm{C}$ & 5.33544800 & -4.40245200 & -0.15604000 \\
\hline $\mathrm{H}$ & 6.24526800 & -3.93452700 & -0.53290800 \\
\hline $\mathrm{H}$ & 5.51638300 & -4.88048800 & 0.81062800 \\
\hline $\mathrm{H}$ & 5.02584300 & -5.19103000 & -0.85213800 \\
\hline $\mathrm{C}$ & -3.05806100 & 1.27380700 & 0.40675900 \\
\hline $\mathrm{H}$ & -3.40740500 & 2.10533200 & 1.03134100 \\
\hline $\mathrm{C}$ & -4.25265900 & 0.59623200 & -0.32257800 \\
\hline $\mathrm{C}$ & -4.24231500 & -0.94417800 & -0.10023500 \\
\hline $\mathrm{C}$ & -4.20339300 & 1.00005400 & -1.81500500 \\
\hline $\mathrm{H}$ & -5.02892000 & 0.59194700 & -2.40227900 \\
\hline $\mathrm{H}$ & -4.25814200 & 2.08876300 & -1.89657400 \\
\hline $\mathrm{H}$ & -3.26791100 & 0.67817300 & -2.28722100 \\
\hline $\mathrm{C}$ & -5.67085500 & 0.88519200 & 0.22658800 \\
\hline
\end{tabular}




$\begin{array}{lrrr}\mathrm{C} & -5.56184400 & -1.41391500 & -0.73714800 \\ \mathrm{C} & -6.54780500 & -0.26277500 & -0.35687100 \\ \mathrm{O} & -6.06503900 & 2.20622000 & -0.17771400 \\ \mathrm{C} & -7.08029100 & 2.90304600 & 0.39711100 \\ \mathrm{O} & -7.31104300 & 4.01361900 & -0.02336800 \\ \mathrm{C} & -7.86397400 & 2.27582200 & 1.53076900 \\ \mathrm{H} & -7.21207100 & 2.04889400 & 2.38173600 \\ \mathrm{H} & -8.63019300 & 2.98297200 & 1.84788300 \\ \mathrm{H} & -8.34097400 & 1.34467200 & 1.20985900 \\ \mathrm{C} & 0.03222300 & -0.21783400 & -0.87987400 \\ \mathrm{H} & 1.05228700 & -0.39750000 & -1.23859600 \\ \mathrm{H} & -0.61782000 & -0.24845400 & -1.75791600 \\ \mathrm{H} & -0.02633000 & 0.79667800 & -0.46518000 \\ \mathrm{H} & -5.87785100 & -2.38329000 & -0.35013800 \\ \mathrm{H} & -2.55569300 & -1.06834700 & -1.42481200 \\ \mathrm{C} & -1.85725100 & -1.06958500 & 0.64748700 \\ \mathrm{C} & -2.22263200 & 0.31388300 & 1.28236900 \\ \mathrm{H} & -1.31797500 & 0.84113700 & 1.61239200 \\ \mathrm{H} & -5.47482200 & -1.52095000 & -1.82221100 \\ \mathrm{H} & 1.87448600 & 1.63787600 & 0.68732500 \\ \mathrm{H} & -4.36647700 & -1.11071100 & 0.98177800 \\ \mathrm{H} & -7.09677200 & 0.10646500 & -1.22811700 \\ \mathrm{H} & -7.28971700 & -0.60176100 & 0.37148100 \\ \mathrm{H} & -5.64940300 & 0.84243700 & 1.32213200 \\ \mathrm{H} & -2.40612100 & 1.73275200 & -0.34667500 \\ \mathrm{H} & -2.77731800 & 0.11083500 & 2.20459200 \\ \mathrm{H} & -1.98694600 & -1.80584800 & 1.45496700 \\ \mathrm{H} & 0.59242000 & -0.13222500 & 1.78404700\end{array}$

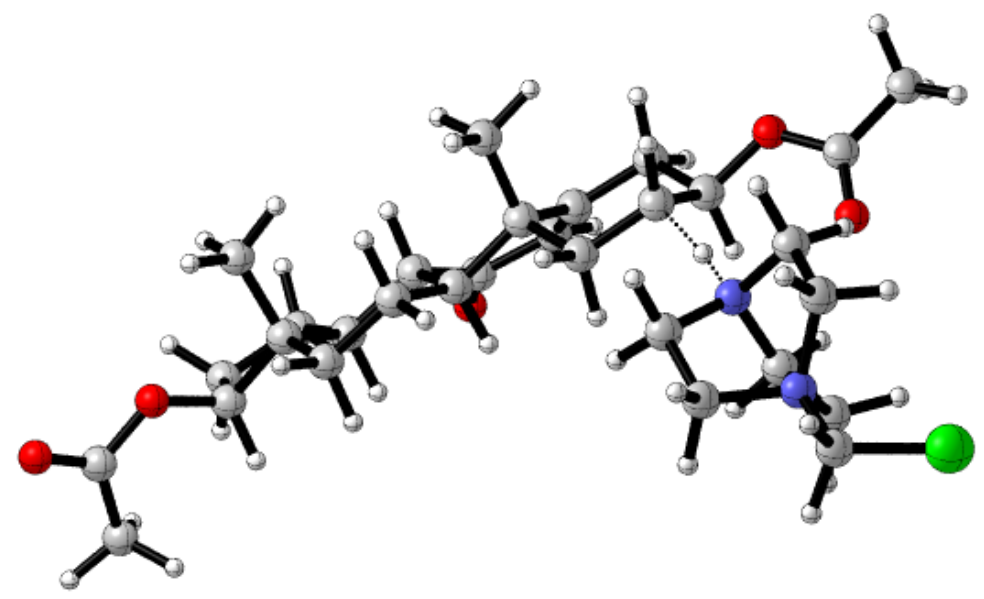

Figure S64. Transition state involving carbon C1 (bottom hydrogen) using SRD. 
Thermal correction to Energy=

Thermal correction to Enthalpy=

Thermal correction to Gibbs Free Energy=

Sum of electronic and zero-point Energies $=$

Sum of electronic and thermal Energies=

Sum of electronic and thermal Enthalpies=

Sum of electronic and thermal Free Energies=
0.772165

0.773109

0.662132

$-2115.342111$

$-2115.304536$

$-2115.303591$

$-2115.414569$

Number of Imaginary Frequencies $=1$

E (Single Point Energy) $\left[\right.$ IEFPCM $\left._{(\mathrm{CH} 3 \mathrm{CN})} \mathrm{U} \omega \mathrm{B} 97 \mathrm{X}-\mathrm{D} / 6-311++\mathrm{G}(2 \mathrm{~d}, 2 \mathrm{p})\right]=-2116.37808574$

22

$\begin{array}{lrrr}\mathrm{C} & 4.95247800 & -0.31420700 & 1.06331800 \\ \mathrm{C} & 3.05984900 & -1.83459600 & 0.79735300 \\ \mathrm{C} & 3.96919500 & -0.68061100 & -1.15077800 \\ \mathrm{C} & 5.82537900 & -1.58923400 & 1.01988400 \\ \mathrm{H} & 4.70104700 & -0.05439800 & 2.09430400 \\ \mathrm{C} & 3.93831800 & -3.02788000 & 0.34546400 \\ \mathrm{H} & 2.05527000 & -1.91921900 & 0.37923600 \\ \mathrm{H} & 2.97683400 & -1.78177100 & 1.88514300 \\ \mathrm{H} & 3.05258400 & -1.00946300 & -1.64570200 \\ \mathrm{H} & 4.23790000 & 0.31292800 & -1.50913600 \\ \mathrm{C} & 5.12588300 & -1.68517700 & -1.35711600 \\ \mathrm{H} & 5.78613900 & -2.16257100 & 1.94928400 \\ \mathrm{H} & 6.86713800 & -1.35661600 & 0.79574900 \\ \mathrm{H} & 3.51478500 & -3.55302100 & -0.51338100 \\ \mathrm{H} & 4.08775800 & -3.74539500 & 1.15525500 \\ \mathrm{H} & 4.92232600 & -2.38100700 & -2.17397800 \\ \mathrm{H} & 6.07661600 & -1.18946700 & -1.55312700 \\ \mathrm{~N} & 3.67982600 & -0.56584700 & 0.31408700 \\ \mathrm{~N} & 5.30495000 & -2.50526100 & -0.08136900 \\ \mathrm{C} & 6.22766800 & -3.70305500 & -0.29734500 \\ \mathrm{H} & 5.73736900 & -4.36076300 & -1.01626100 \\ \mathrm{H} & 6.33869900 & -4.20159300 & 0.66647700 \\ \mathrm{O} & -3.54688300 & 2.87164300 & -2.15502400 \\ \mathrm{Cl} & 7.81929600 & -3.24466600 & -0.90854200 \\ \mathrm{C} & -2.84363500 & 2.54885900 & -1.21358700 \\ \mathrm{C} & -1.51698100 & 3.18932900 & -1.03953800 \\ \mathrm{C} & -3.22895200 & 1.50026400 & -0.17254700 \\ \mathrm{C} & -0.59065200 & 2.75412000 & -0.16306200 \\ \mathrm{C} & -0.78415600 & 1.50643300 & 0.68323200 \\ \mathrm{C} & 0.67969800 & 3.53586500 & 0.03448300 \\ \mathrm{C} & 0.56274100 & 0.64665600 & 0.52637200 \\ \mathrm{C} & 1.96612100 & 2.65513700 & -0.08247700\end{array}$




$\begin{array}{lrrr}\mathrm{H} & 0.70375800 & 3.98948000 & 1.03286200 \\ \mathrm{H} & 0.75895100 & 4.35206800 & -0.68959200 \\ \mathrm{C} & 1.81321800 & 1.43711900 & 0.78730100 \\ \mathrm{H} & 0.55753300 & 0.26429000 & -0.50401300 \\ \mathrm{H} & 2.12316000 & 2.38638900 & -1.13273400 \\ \mathrm{H} & 2.90185200 & 0.38084500 & 0.49435600 \\ \mathrm{H} & 2.04165100 & 1.63128100 & 1.84111900 \\ \mathrm{H} & -1.33767100 & 4.05840800 & -1.66835800 \\ \mathrm{O} & 3.02820500 & 3.54050200 & 0.33581000 \\ \mathrm{C} & 4.29460400 & 3.25431000 & -0.00446100 \\ \mathrm{O} & 4.61142600 & 2.19123400 & -0.52419300 \\ \mathrm{C} & 5.23374600 & 4.38104700 & 0.31515700 \\ \mathrm{H} & 5.01098300 & 4.80884400 & 1.29625000 \\ \mathrm{H} & 6.26623600 & 4.03366100 & 0.26933700 \\ \mathrm{H} & 5.09601900 & 5.17860500 & -0.42490300 \\ \mathrm{C} & -3.49583300 & -1.41779900 & 0.51182900 \\ \mathrm{H} & -3.25771600 & -1.86755100 & -0.46356200 \\ \mathrm{C} & -4.72762400 & -0.51516400 & 0.36780200 \\ \mathrm{C} & -4.39928700 & 0.61800000 & -0.63372600 \\ \mathrm{C} & -5.19564200 & -0.00241400 & 1.74911800 \\ \mathrm{H} & -6.09925200 & 0.60801300 & 1.67775500 \\ \mathrm{H} & -5.43269900 & -0.84934500 & 2.39817000 \\ \mathrm{H} & -4.43598800 & 0.60495500 & 2.25021700 \\ \mathrm{C} & -5.92728900 & -1.15494900 & -0.37156100 \\ \mathrm{H} & -5.77261200 & 1.25789700 & -0.93939500 \\ \mathrm{H} & -6.76162500 & 0.05392400 & -0.87727400 \\ \mathrm{C} & -6.62239100 & -2.02594400 & 0.53070200 \\ \mathrm{O} & -7.45793900 & -3.02572000 & 0.12928100 \\ \mathrm{C} & -7.97421600 & -3.70613100 & 0.98266200 \\ \mathrm{O} & -7.68688900 & -3.24129300 & -1.35258400 \\ \mathrm{C} & -8.40617500 & -4.05181300 & -1.46905800 \\ \mathrm{H} & -8.07810600 & -2.33814000 & -1.83091300 \\ \mathrm{H} & -6.75518900 & -3.51681000 & -1.85967600 \\ \mathrm{H} & -0.96462000 & 1.89095300 & 2.17136800 \\ \mathrm{H} & -0.19586700 & 2.58969700 & 2.51248900 \\ \mathrm{H} & -1.93227900 & 2.37617500 & 2.32923500 \\ \mathrm{H} & -0.92194300 & 1.01144400 & 2.82034600 \\ \mathrm{H} & -5.77865700 & 1.75866500 & -1.90687300 \\ \mathrm{H} & -3.51928200 & 2.05424500 & 0.73292300 \\ \mathrm{H} & -286616200 & 0.62891400 & 0.13728700 \\ \mathrm{H} & -0.60641400 & 1.01341900 \\ \mathrm{H} & -2.47440000 & -0.29885800 & 2.04608400 \\ \mathrm{H} & 2.01250900 & -0.18747800 \\ \mathrm{H} & 0.54338500 & 0.59408700 \\ \mathrm{H} & 0.12837700 & -1.56295300 \\ \mathrm{H} & 0.24059800 & -0.18245700\end{array}$




$\begin{array}{lrrr}\mathrm{H} & -7.20606900 & -0.14949100 & -1.85552900 \\ \mathrm{H} & -5.54823000 & -1.74140300 & -1.21751100 \\ \mathrm{H} & -3.69234300 & -2.24419200 & 1.20497200 \\ \mathrm{H} & -1.40809800 & -1.26822400 & 1.04702500 \\ \mathrm{H} & -1.63482200 & 0.24593900 & -0.84179400 \\ \mathrm{H} & 0.48580200 & -0.20492600 & 1.20954900\end{array}$

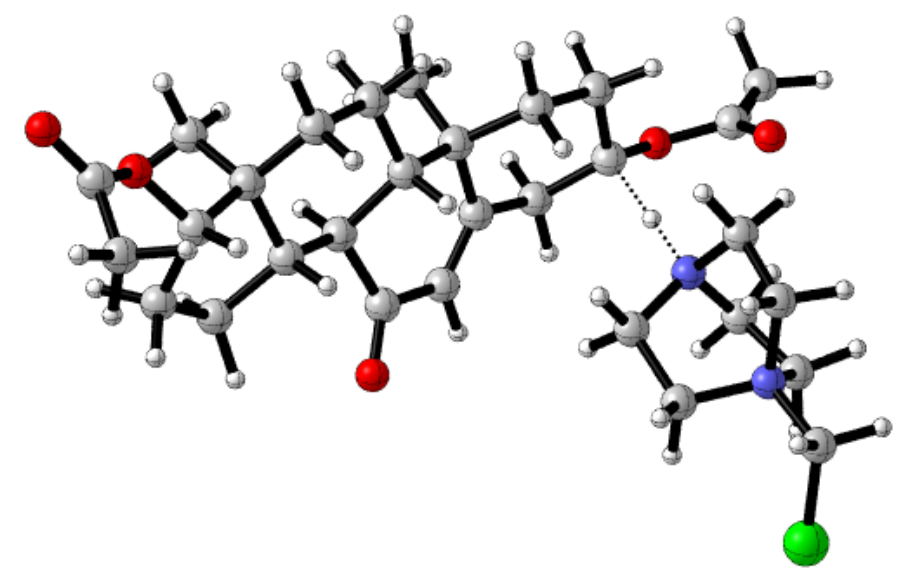

Figure S65. Transition state involving carbon C4 using SRD.

Zero-point correction $=$

Thermal correction to Energy=

Thermal correction to Enthalpy=

Thermal correction to Gibbs Free Energy=

Sum of electronic and zero-point Energies $=$

Sum of electronic and thermal Energies=

Sum of electronic and thermal Enthalpies=

Sum of electronic and thermal Free Energies=
0.734273 (Hartree/Particle)

0.771912

0.772856

0.662372

$-2115.341597$

$-2115.303958$

$-2115.303014$

$-2115.413498$

Number of Imaginary Frequencies $=1$

E (Single Point Energy) $\left[\right.$ IEFPCM $\left._{(\mathrm{CH} 3 \mathrm{CN})} \mathrm{U \omega B} 97 \mathrm{X}-\mathrm{D} / 6-311++\mathrm{G}(2 \mathrm{~d}, 2 \mathrm{p})\right]=-2116.38032235$

22

$\mathrm{C}$

4.70907300

0.34102400

0.48753400

C

3.64101900

0.40505900

$-1.70926500$

$\mathrm{C}$

2.42753700

1.18627000

0.26005600

C

5.36836100

1.68019000

0.08315200

$\mathrm{H}$

5.35047400

$\begin{array}{ll}-0.49801700 & 0.21955300\end{array}$

C

3.98312600

$1.89674600-1.94908900$

$\mathrm{H}$

2.72868900

$0.13241600-2.24339100$

$\mathrm{H}$

4.43739800

$-0.26848000-2.02258600$

$\mathrm{H}$

1.54068600

$1.13483100-0.37363300$ 


\begin{tabular}{|c|c|c|c|}
\hline $\mathrm{H}$ & 2.12492900 & 0.91242200 & 1.27134000 \\
\hline $\mathrm{C}$ & 3.07120000 & 2.59409200 & 0.23792100 \\
\hline $\mathrm{H}$ & 6.19006200 & 1.54010200 & -0.62303000 \\
\hline $\mathrm{H}$ & 5.73878500 & 2.23085100 & 0.94864100 \\
\hline $\mathrm{H}$ & 3.14420300 & 2.45776000 & -2.36685400 \\
\hline $\mathrm{H}$ & 4.84318700 & 2.00637600 & -2.61342600 \\
\hline $\mathrm{H}$ & 2.40053300 & 3.33952700 & -0.19484900 \\
\hline $\mathrm{H}$ & 3.36742200 & 2.93366900 & 1.23043600 \\
\hline $\mathrm{N}$ & 3.41584700 & 0.18527900 & -0.24739100 \\
\hline $\mathrm{N}$ & 4.33287400 & 2.55096900 & -0.61623500 \\
\hline $\mathrm{C}$ & 4.87753600 & 3.94927700 & -0.89987500 \\
\hline $\mathrm{H}$ & 4.12394200 & 4.47408100 & -1.48833300 \\
\hline $\mathrm{H}$ & 5.79639400 & 3.82558500 & -1.47438300 \\
\hline $\mathrm{O}$ & -1.35722200 & 1.21658600 & 2.51348000 \\
\hline $\mathrm{Cl}$ & 5.22183600 & 4.86682400 & 0.56925900 \\
\hline $\mathrm{C}$ & -1.25755900 & 0.11632400 & 1.99368200 \\
\hline $\mathrm{C}$ & 0.01046800 & -0.64411800 & 2.12333200 \\
\hline $\mathrm{C}$ & -2.33229800 & -0.54931300 & 1.14743200 \\
\hline $\mathrm{C}$ & 0.38321800 & -1.60231100 & 1.25796100 \\
\hline $\mathrm{C}$ & -0.46888600 & -2.02794300 & 0.05918400 \\
\hline $\mathrm{C}$ & 1.70179500 & -2.32073800 & 1.46161300 \\
\hline $\mathrm{C}$ & 0.45034200 & -2.02726600 & -1.20135200 \\
\hline $\mathrm{C}$ & 2.53382400 & -2.41379300 & 0.20054000 \\
\hline $\mathrm{H}$ & 1.51026500 & -3.36014700 & 1.78158200 \\
\hline $\mathrm{H}$ & 2.27668200 & -1.87448800 & 2.28208700 \\
\hline $\mathrm{C}$ & 1.76972400 & -2.80137300 & -1.05339600 \\
\hline $\mathrm{H}$ & 0.66902500 & -0.98197200 & -1.47126500 \\
\hline $\mathrm{H}$ & 2.99770500 & -1.00955500 & -0.06322000 \\
\hline $\mathrm{H}$ & 1.56378100 & -3.88039000 & -0.96243900 \\
\hline $\mathrm{H}$ & 0.64529900 & -0.35373900 & 2.96017900 \\
\hline $\mathrm{O}$ & 3.67679000 & -3.18007000 & 0.46547100 \\
\hline $\mathrm{C}$ & 4.74472100 & -3.19994100 & -0.40228800 \\
\hline $\mathrm{O}$ & 4.91691600 & -2.30952000 & -1.20991900 \\
\hline $\mathrm{C}$ & 5.61930700 & -4.39273200 & -0.17450300 \\
\hline $\mathrm{H}$ & 5.10562300 & -5.29196700 & -0.53576300 \\
\hline $\mathrm{H}$ & 6.55641400 & -4.27247700 & -0.71831600 \\
\hline $\mathrm{H}$ & 5.80609700 & -4.53722000 & 0.89376400 \\
\hline $\mathrm{C}$ & -3.85271400 & -0.53173300 & -1.44459900 \\
\hline $\mathrm{H}$ & -3.49565700 & 0.41626400 & -1.87429600 \\
\hline $\mathrm{C}$ & -4.55171100 & -0.25243100 & -0.10722900 \\
\hline $\mathrm{C}$ & -3.52174800 & 0.37508900 & 0.86455200 \\
\hline $\mathrm{C}$ & -5.21695700 & -1.53425700 & 0.44474100 \\
\hline $\mathrm{H}$ & -5.76495100 & -1.34916100 & 1.37221200 \\
\hline $\mathrm{H}$ & -5.93762700 & -1.92054600 & -0.28047300 \\
\hline $\mathrm{H}$ & -4.49121300 & -2.32625600 & 0.65078200 \\
\hline $\mathrm{C}$ & -5.59264600 & 0.89236200 & -0.13186500 \\
\hline
\end{tabular}




$\begin{array}{lrrr}\mathrm{C} & -4.37943200 & 0.90378400 & 2.03442700 \\ \mathrm{C} & -5.70163200 & 1.35867900 & 1.34600700 \\ \mathrm{O} & -6.82431900 & 0.39855400 & -0.68133400 \\ \mathrm{C} & -7.78287000 & 1.19463500 & -1.22015900 \\ \mathrm{O} & -8.79190700 & 0.66874100 & -1.63032900 \\ \mathrm{C} & -7.54827900 & 2.68920500 & -1.30486500 \\ \mathrm{H} & -8.44673200 & 3.15094300 & -1.71396800 \\ \mathrm{H} & -7.33595100 & 3.11645300 & -0.32009400 \\ \mathrm{H} & -6.70227000 & 2.91157600 & -1.96515800 \\ \mathrm{C} & -0.99582800 & -3.46421500 & 0.32376000 \\ \mathrm{H} & -0.19579600 & -4.15308900 & 0.61224000 \\ \mathrm{H} & -1.73053900 & -3.47228400 & 1.13425700 \\ \mathrm{H} & -1.47007200 & -3.87989000 & -0.56905200 \\ \mathrm{H} & -3.88112700 & 1.71422500 & 2.56724000 \\ \mathrm{H} & -2.67095500 & -1.43485300 & 1.70566600 \\ \mathrm{C} & -1.64520600 & -1.01068300 & -0.18294400 \\ \mathrm{C} & -2.66281900 & -1.49148900 & -1.24828700 \\ \mathrm{H} & -3.05258200 & -2.47565600 & -0.97461900 \\ \mathrm{H} & -4.57451800 & 0.11123800 & 2.76533600 \\ \mathrm{H} & 4.49400600 & 0.30216500 & 1.55761800 \\ \mathrm{H} & -3.10626300 & 1.25894200 & 0.35224200 \\ \mathrm{H} & -6.57918700 & 0.89590600 & 1.80661900 \\ \mathrm{H} & -5.83802300 & 2.44194100 & 1.41307700 \\ \mathrm{H} & -5.21495500 & 1.70195100 & -0.76768700 \\ \mathrm{H} & -4.54914000 & -0.96617900 & -2.17106800 \\ \mathrm{H} & -2.15038000 & -1.62269300 & -2.20918000 \\ \mathrm{H} & -1.17704300 & -0.09807900 & -0.59339100 \\ \mathrm{H} & -0.09415700 & -2.44545000 & -2.05299900 \\ \mathrm{H} & 2.38921800 & -2.69111400 & -1.94941800\end{array}$

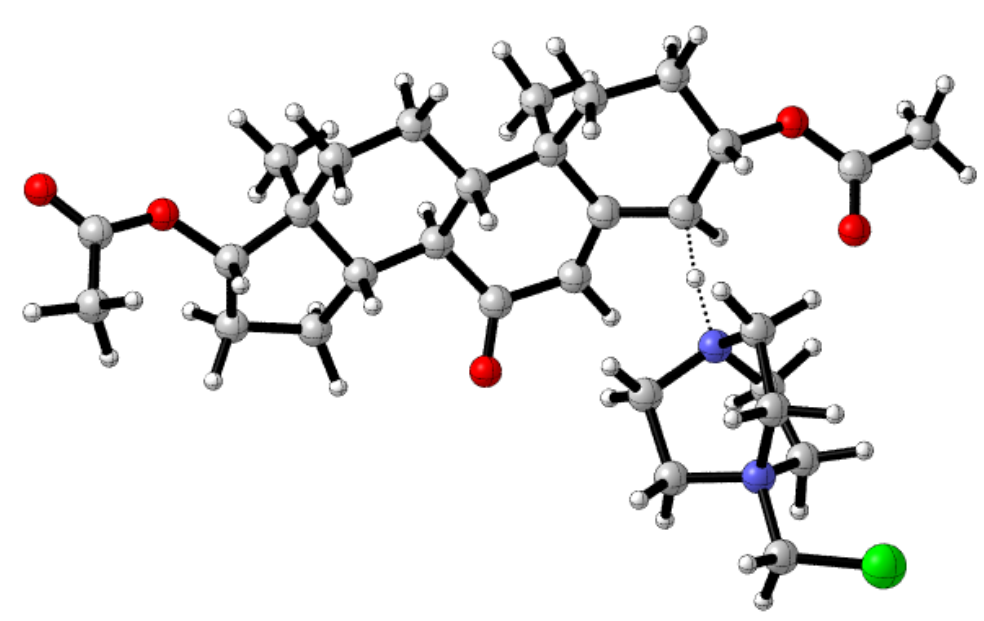

Figure S66. Transition state involving carbon C13 (bottom hydrogen) using SRD. 
Zero-point correction=

Thermal correction to Energy=

Thermal correction to Enthalpy=

Thermal correction to Gibbs Free Energy=

Sum of electronic and zero-point Energies $=$

Sum of electronic and thermal Energies=

Sum of electronic and thermal Enthalpies=

Sum of electronic and thermal Free Energies=

Number of Imaginary Frequencies $=1$
0.734751 (Hartree/Particle)

0.772210

0.773154

0.663190

$-2115.354894$

$-2115.317434$

$-2115.316490$

$-2115.426454$

E (Single Point Energy) IEFPCM $_{(\mathrm{CH} 3 \mathrm{CN})} \mathrm{U \omega B}$ B7X-D/6-311++G(2d,2p)] $=-2116.37545493$

22

C $\quad-3.68138200 \quad-0.58645600-1.28116300$

$\begin{array}{llll}\mathrm{C} & -2.01388300 & -1.67118900 & 0.12223200\end{array}$

$\begin{array}{llll}\mathrm{C} & -3.97071400 & -0.62960200 & 1.13479400\end{array}$

C $\quad-4.27957000 \quad-1.99986800-1.49177400$

$\mathrm{H} \quad-2.96451600 \quad-0.36037100 \quad-2.07402900$

C $\quad-2.80580900 \quad-3.00095300 \quad 0.22160500$

$\mathrm{H} \quad-1.36990000 \quad-1.54869600 \quad 0.99709100$

$\mathrm{H} \quad-1.38088800 \quad-1.65256600-0.76794900$

$\mathrm{H} \quad-3.42312300 \quad-0.71147600 \quad 2.07673700$

$\mathrm{H} \quad-4.55554800 \quad 0.29166200 \quad 1.13477700$

C $\quad-4.88596200 \quad-1.85684500 \quad 0.90602300$

$\mathrm{H} \quad-3.70169000 \quad-2.60234000 \quad-2.19672900$

$\mathrm{H} \quad-\quad-5.31448200 \quad-1.95769300 \quad-1.83321300$

$\mathrm{H} \quad \begin{array}{llll}\mathrm{H} & -2.81162800 & -3.40334000 & 1.23697300\end{array}$

$\mathrm{H} \quad-2.40762600 \quad-3.76135900 \quad-0.45376500$

$\mathrm{H} \quad-\quad-5.00168800 \quad-2.45898300 \quad 1.80987400$

$\mathrm{H} \quad-\quad-5.87591900 \quad-1.57536100 \quad 0.54712600$

$\mathrm{N} \quad-2.97307200 \quad-0.53679100 \quad 0.03002900$

$\mathrm{N} \quad-4.25967300 \quad-2.74581800 \quad-0.16344900$

$\begin{array}{llll}\mathrm{C} & -4.96510300 & -4.09375100 & -0.26679800\end{array}$

$\mathrm{H} \quad-4.82338400 \quad-4.60529600 \quad 0.68599700$

$\mathrm{H} \quad-4.48594800 \quad-4.64755700 \quad-1.07498500$

$\begin{array}{llll}\mathrm{O} & 0.94848600 & -1.18117600 & 2.12136900\end{array}$

$\mathrm{Cl} \quad-6.69372100 \quad-3.94758500 \quad-0.60261000$

$\begin{array}{llll}\mathrm{C} & 1.05989600 & -0.02651500 & 1.71244700\end{array}$

$\begin{array}{llll}\text { C } & -0.15287500 & 0.81208800 & 1.76464700\end{array}$

$\begin{array}{llll}\mathrm{C} & 2.27056200 & 0.54567100 & 1.00889900\end{array}$

$\begin{array}{llll}\mathrm{C} & -0.46088100 & 1.70640400 & 0.73515200\end{array}$

$\begin{array}{llll}\mathrm{C} & 0.66535700 & 2.28152600 & -0.11027900\end{array}$

$\begin{array}{llll}\mathrm{C} & -1.84686700 & 1.95034700 & 0.47250700\end{array}$

$\begin{array}{llll}\mathrm{C} & 0.09812300 & 2.80692200 & -1.45377900\end{array}$

$\begin{array}{llll}\mathrm{C} & -2.30246200 & 2.85059900 & -0.67979100\end{array}$

$\begin{array}{llll}\mathrm{H} & -2.47319000 & 2.00176900 & 1.36999300\end{array}$ 


\begin{tabular}{|c|c|c|c|}
\hline $\mathrm{H}$ & -2.33744800 & 0.66544200 & 0.17657300 \\
\hline $\mathrm{C}$ & -1.16055000 & 3.66964200 & -1.27944000 \\
\hline $\mathrm{H}$ & -0.12329300 & 1.95579400 & -2.11567100 \\
\hline $\mathrm{H}$ & -0.96094000 & 4.52480700 & -0.62700900 \\
\hline $\mathrm{H}$ & -0.91546500 & 0.49675600 & 2.47552800 \\
\hline $\mathrm{O}$ & -3.31502000 & 3.77800800 & -0.22592300 \\
\hline $\mathrm{C}$ & -4.55931600 & 3.29417800 & -0.03155000 \\
\hline $\mathrm{O}$ & -4.81750000 & 2.10153200 & -0.13087900 \\
\hline $\mathrm{C}$ & -5.54259000 & 4.37898600 & 0.29621900 \\
\hline $\mathrm{H}$ & -5.74687300 & 4.96512100 & -0.60762600 \\
\hline $\mathrm{H}$ & -6.47363500 & 3.94039500 & 0.65572800 \\
\hline $\mathrm{H}$ & -5.12804300 & 5.06584900 & 1.03928400 \\
\hline $\mathrm{C}$ & 4.07249000 & 0.64017900 & -1.38405200 \\
\hline $\mathrm{H}$ & 3.69281800 & -0.20847300 & -1.97229900 \\
\hline $\mathrm{C}$ & 4.57045800 & 0.13386900 & -0.02362800 \\
\hline $\mathrm{C}$ & 3.37511200 & -0.48531500 & 0.74571500 \\
\hline $\mathrm{C}$ & 5.28311500 & 1.26418400 & 0.75345900 \\
\hline $\mathrm{H}$ & 5.66285300 & 0.92857600 & 1.72207500 \\
\hline $\mathrm{H}$ & 6.14220000 & 1.61978600 & 0.17956100 \\
\hline $\mathrm{H}$ & 4.62944200 & 2.12150400 & 0.93922400 \\
\hline $\mathrm{C}$ & 5.49356500 & -1.10883500 & -0.07900000 \\
\hline $\mathrm{C}$ & 4.03142100 & -1.23621100 & 1.92520100 \\
\hline $\mathrm{C}$ & 5.37924100 & -1.74738500 & 1.33246200 \\
\hline $\mathrm{O}$ & 6.82105800 & -0.69519100 & -0.42345000 \\
\hline $\mathrm{C}$ & 7.75543800 & -1.52213000 & -0.97139800 \\
\hline $\mathrm{O}$ & 8.85222700 & -1.06638900 & -1.18786800 \\
\hline $\mathrm{C}$ & 7.37592100 & -2.95141500 & -1.30010500 \\
\hline $\mathrm{H}$ & 8.26614900 & -3.46083000 & -1.66870300 \\
\hline $\mathrm{H}$ & 6.99416000 & -3.47873300 & -0.42039700 \\
\hline $\mathrm{H}$ & 6.60462300 & -2.97887600 & -2.07839500 \\
\hline $\mathrm{C}$ & 1.21372000 & 3.48757300 & 0.71933100 \\
\hline $\mathrm{H}$ & 0.42525700 & 4.20582100 & 0.95718600 \\
\hline $\mathrm{H}$ & 1.65394400 & 3.17318600 & 1.66840200 \\
\hline $\mathrm{H}$ & 1.97938400 & 4.01464600 & 0.14318900 \\
\hline $\mathrm{H}$ & 3.40047100 & -2.04651100 & 2.29351000 \\
\hline $\mathrm{H}$ & 2.66588300 & 1.33953200 & 1.65745800 \\
\hline $\mathrm{C}$ & 1.77610300 & 1.19093700 & -0.33909500 \\
\hline $\mathrm{C}$ & 2.95078500 & 1.68587200 & -1.21624000 \\
\hline $\mathrm{H}$ & 3.37420800 & 2.60208300 & -0.79254100 \\
\hline $\mathrm{H}$ & 4.20917400 & -0.56074700 & 2.76923100 \\
\hline $\mathrm{H}$ & -4.44315700 & 0.19369000 & -1.26698200 \\
\hline $\mathrm{H}$ & 2.93413400 & -1.25006000 & 0.08469300 \\
\hline $\mathrm{H}$ & 6.23266700 & -1.43252400 & 1.93953900 \\
\hline $\mathrm{H}$ & 5.40840900 & -2.83960400 & 1.28045800 \\
\hline $\mathrm{H}$ & 5.11624400 & -1.79512700 & -0.84662600 \\
\hline $\mathrm{H}$ & 4.89142700 & 1.08302200 & -1.96205800 \\
\hline
\end{tabular}




$\begin{array}{lrrr}\mathrm{H} & 2.56964400 & 1.95316900 & -2.20764800 \\ \mathrm{H} & 1.28979300 & 0.37410900 & -0.90074600 \\ \mathrm{H} & 0.86197400 & 3.39805000 & -1.96500100 \\ \mathrm{H} & -1.47917700 & 4.07872900 & -2.24292300 \\ \mathrm{H} & -2.75888200 & 2.24093100 & -1.46820500\end{array}$

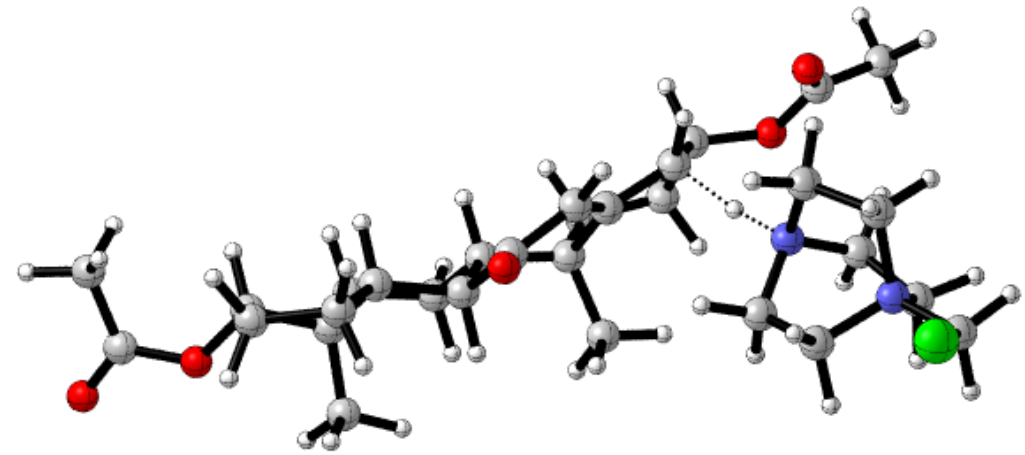

Figure S67. Transition state involving carbon C13 (top hydrogen) using SRD.

Zero-point correction=

Thermal correction to Energy=

Thermal correction to Enthalpy=

Thermal correction to Gibbs Free Energy=

Sum of electronic and zero-point Energies=

Sum of electronic and thermal Energies=

Sum of electronic and thermal Enthalpies=

Sum of electronic and thermal Free Energies=
0.734689 (Hartree/Particle)

0.772249

0.773193

0.662222

$-2115.349986$

$-2115.312427$

$-2115.311482$

$-2115.422453$

Number of Imaginary Frequencies $=1$

E (Single Point Energy) $\left[\operatorname{IEFPCM}_{(\mathrm{CH} 3 \mathrm{CN})} \mathrm{U} \omega \mathrm{B} 97 \mathrm{X}-\mathrm{D} / 6-311++\mathrm{G}(2 \mathrm{~d}, 2 \mathrm{p})\right]=-2116.37872335$

22

$\begin{array}{lrrr}\mathrm{C} & -2.91545800 & -1.22331000 & 0.79549600 \\ \mathrm{C} & -4.60752100 & 0.53023700 & 0.86575900 \\ \mathrm{C} & -4.05633800 & -0.54033200 & -1.24997000 \\ \mathrm{C} & -3.94241900 & -2.38162400 & 0.84495500 \\ \mathrm{H} & -2.63696700 & -0.88259200 & 1.79517000 \\ \mathrm{C} & -5.56992900 & -0.59187600 & 1.32568600 \\ \mathrm{H} & -5.11310300 & 1.25891200 & 0.23305500 \\ \mathrm{H} & -4.17907600 & 1.05170300 & 1.72440900 \\ \mathrm{H} & -4.30320700 & 0.34038500 & -1.84655200 \\ \mathrm{H} & -3.27138000 & -1.10504800 & -1.75761200 \\ \mathrm{C} & -5.30481900 & -1.41378700 & -0.99016100 \\ \mathrm{H} & -4.01540600 & -2.82115800 & 1.84214600 \\ \mathrm{H} & -3.71261900 & -3.17533500 & 0.13413000\end{array}$




\begin{tabular}{|c|c|c|c|}
\hline $\mathrm{H}$ & -6.61509700 & -0.30181500 & 1.19694800 \\
\hline $\mathrm{H}$ & -5.41285300 & -0.88097600 & 2.36729700 \\
\hline $\mathrm{H}$ & -6.23700300 & -0.87233600 & -1.16800100 \\
\hline $\mathrm{H}$ & -5.30472500 & -2.32016600 & -1.59678900 \\
\hline $\mathrm{N}$ & -3.50686700 & -0.06965300 & 0.05964000 \\
\hline $\mathrm{N}$ & -5.31692100 & -1.83407000 & 0.47704500 \\
\hline$c$ & -6.42692300 & -2.84166400 & 0.76218500 \\
\hline $\mathrm{H}$ & -7.36245500 & -2.39249500 & 0.42654300 \\
\hline 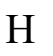 & -6.44444200 & -3.00573300 & 1.84039600 \\
\hline $\mathrm{O}$ & 1.09253300 & -2.11874700 & -1.35479400 \\
\hline $\mathrm{Cl}$ & -6.18724500 & -4.38439000 & -0.06393300 \\
\hline $\mathrm{C}$ & 1.14607500 & -0.96441600 & -0.93570500 \\
\hline 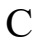 & 0.00215500 & -0.09357600 & -1.23013000 \\
\hline $\mathrm{C}$ & 2.24787900 & -0.43450600 & -0.03387000 \\
\hline $\mathrm{C}$ & -0.33156400 & 1.02330800 & -0.46496500 \\
\hline $\mathrm{C}$ & 0.61862500 & 1.48062400 & 0.64530000 \\
\hline $\mathrm{C}$ & -1.56367100 & 1.69351900 & -0.76294800 \\
\hline $\mathrm{C}$ & 0.50307500 & 3.02219400 & 0.79220800 \\
\hline $\mathrm{C}$ & -1.74289800 & 3.15135300 & -0.35518200 \\
\hline $\mathrm{H}$ & -1.95179400 & 1.47795200 & -1.76277600 \\
\hline $\mathrm{H}$ & -2.57856800 & 0.80261000 & -0.18834600 \\
\hline $\mathrm{C}$ & -0.93923200 & 3.52738000 & 0.88400100 \\
\hline $\mathrm{H}$ & 1.00055000 & 3.49939400 & -0.06300000 \\
\hline $\mathrm{H}$ & -1.44362200 & 3.11950300 & 1.76837100 \\
\hline $\mathrm{H}$ & -0.66186700 & -0.45085000 & -2.01542400 \\
\hline $\mathrm{O}$ & -3.10456200 & 3.59426800 & -0.06433300 \\
\hline $\mathrm{C}$ & -4.11676500 & 3.42594900 & -0.94282700 \\
\hline $\mathrm{O}$ & -4.13414600 & 2.54566400 & -1.78809700 \\
\hline $\mathrm{C}$ & -5.21177100 & 4.43435400 & -0.72218700 \\
\hline $\mathrm{H}$ & -4.90636900 & 5.38493400 & -1.17668400 \\
\hline $\mathrm{H}$ & -6.13324700 & 4.10264400 & -1.20251300 \\
\hline $\mathrm{H}$ & -5.36784200 & 4.62635800 & 0.34275200 \\
\hline $\mathrm{C}$ & 4.60435700 & 1.27168800 & 0.63356700 \\
\hline $\mathrm{H}$ & 4.79581600 & 1.80647000 & -0.30784900 \\
\hline $\mathrm{C}$ & 4.75582500 & -0.23870600 & 0.40111200 \\
\hline $\mathrm{C}$ & 3.65949300 & -0.70867400 & -0.59555600 \\
\hline $\mathrm{C}$ & 4.73439000 & -1.00186400 & 1.74370300 \\
\hline $\mathrm{H}$ & 4.82156600 & -2.08368200 & 1.61128100 \\
\hline $\mathrm{H}$ & 5.58099400 & -0.68482700 & 2.35722600 \\
\hline $\mathrm{H}$ & 3.82113600 & -0.81303200 & 2.31652300 \\
\hline $\mathrm{C}$ & 6.00672300 & -0.65974300 & -0.41258800 \\
\hline $\mathrm{C}$ & 4.08874000 & -2.13303500 & -0.99536100 \\
\hline $\mathrm{C}$ & 5.64554000 & -2.05204400 & -1.00375000 \\
\hline $\mathrm{O}$ & 7.14792700 & -0.67848300 & 0.44967500 \\
\hline $\mathrm{C}$ & 8.43574000 & -0.56851400 & 0.00735100 \\
\hline $\mathrm{O}$ & 9.31873100 & -0.66579600 & 0.8237150 \\
\hline
\end{tabular}




$\begin{array}{lrrr}\mathrm{C} & 8.68873700 & -0.31000300 & -1.46322400 \\ \mathrm{H} & 9.76656800 & -0.30909200 & -1.62484400 \\ \mathrm{H} & 8.22893600 & -1.07816400 & -2.09295600 \\ \mathrm{H} & 8.28513200 & 0.66416100 & -1.76218800 \\ \mathrm{C} & 0.21458900 & 0.79361300 & 1.97641500 \\ \mathrm{H} & -0.80050300 & 1.07620000 & 2.27782400 \\ \mathrm{H} & 0.25476200 & -0.29844100 & 1.90828800 \\ \mathrm{H} & 0.88617600 & 1.10045000 & 2.78320000 \\ \mathrm{H} & 3.68060100 & -2.42996400 & -1.96458800 \\ \mathrm{H} & 2.14823900 & -0.98783800 & 0.91049800 \\ \mathrm{C} & 2.07750100 & 1.08987200 & 0.20781600 \\ \mathrm{C} & 3.19030300 & 1.63715000 & 1.13564800 \\ \mathrm{H} & 3.05191800 & 1.25960700 & 2.15547500 \\ \mathrm{H} & 3.73733600 & -2.87105800 & -0.26803900 \\ \mathrm{H} & -2.00723200 & -1.53922200 & 0.27829500 \\ \mathrm{H} & 3.76232100 & -0.07601800 & -1.49237800 \\ \mathrm{H} & 6.09105300 & -2.83350000 & -0.38166100 \\ \mathrm{H} & 6.05153400 & -2.17324400 & -2.01206300 \\ \mathrm{H} & 6.16651200 & 0.07054500 & -1.21429700 \\ \mathrm{H} & 5.34615800 & 1.63083500 & 1.35602600 \\ \mathrm{H} & 3.11797100 & 2.72732400 & 1.19680400 \\ \mathrm{H} & 2.22616100 & 1.57317500 & -0.77087700 \\ \mathrm{H} & 1.04775000 & 3.34406500 & 1.68380100 \\ \mathrm{H} & -0.95194100 & 4.61549100 & 0.99858300 \\ \mathrm{H} & -1.41469200 & 3.75791000 & -1.21154200\end{array}$

Table S6. Single point energies of all structures calculated at the $\operatorname{IEFPCM}_{(\mathrm{CH} 3 \mathrm{CN})} \mathrm{U \omega B} 97 \mathrm{X}-\mathrm{D} / 6$ $311++\mathrm{G}(2 \mathrm{~d}, 2 \mathrm{p})$ level of theory and thermal correction to Gibbs free energy and Gibbs free energy calculated at the UB3LYP/6-31G(d) level of theory. All energies are reported in Hartrees.

\begin{tabular}{|c|c|c|c|c|}
\hline Structure & 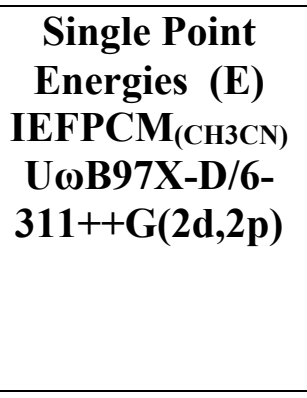 & $\begin{array}{c}\text { Thermal } \\
\text { Corrections } \\
\text { to Gibbs } \\
\text { Free } \\
\text { Energies } \\
\text { (G) } \\
\text { UB3LYP/6- } \\
\text { 31G(d) } \\
\end{array}$ & $\begin{array}{c}\text { Gibbs Free } \\
\text { Energies } \\
\text { (G) } \\
\text { UB3LYP/6- } \\
\text { 31G(d) }\end{array}$ & 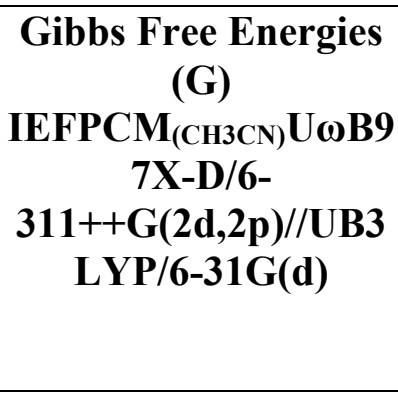 \\
\hline C46 Trans (B) & -1961.73997276 & 0.634695 & -1961.093241 & -1961.105278 \\
\hline C46 Trans (T) & -1961.74463510 & 0.634696 & -1961.094833 & -1961.109939 \\
\hline C46 Cis (B) & -1961.74049618 & 0.633998 & -1961.096888 & -1961.106498 \\
\hline C46 Cis (T) & -1961.74232521 & 0.633947 & -1961.098413 & -1961.108378 \\
\hline
\end{tabular}




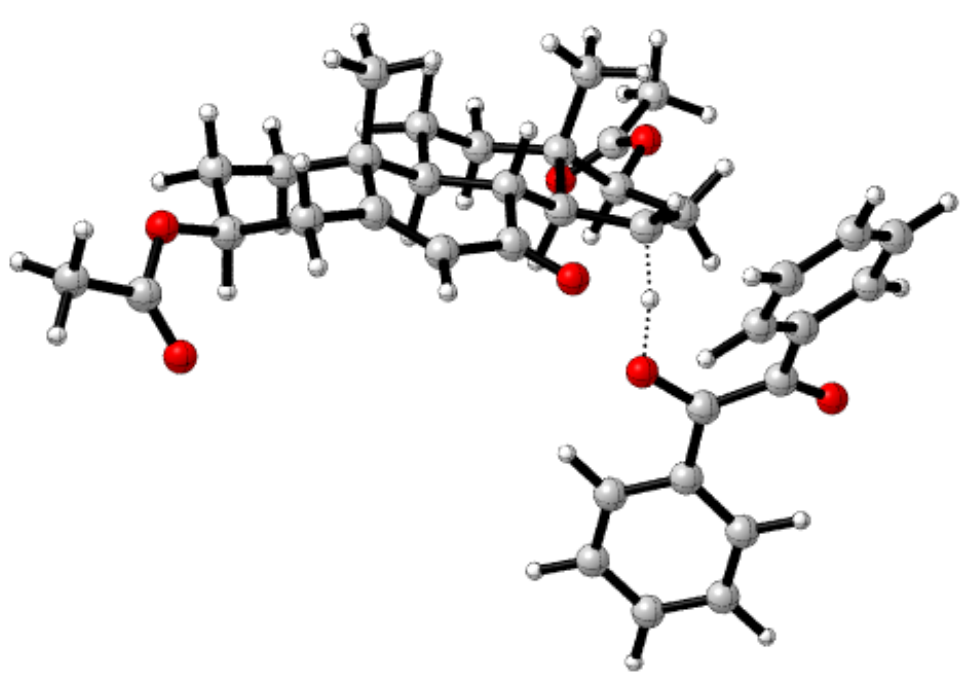

Figure S68. Transition state involving carbon C46 using trans benzil (bottom hydrogen).

Zero-point correction $=$

Thermal correction to Energy=

Thermal correction to Enthalpy=

Thermal correction to Gibbs Free Energy=

Sum of electronic and zero-point Energies $=$

Sum of electronic and thermal Energies=

Sum of electronic and thermal Enthalpies=

Sum of electronic and thermal Free Energies=
0.715666 (Hartree/Particle)

0.757272

0.758216

0.634695

$-1961.012269$

$-1960.970664$

$-1960.969719$

$-1961.093241$

Number of Imaginary Frequencies $=1$

E (Single Point Energy) $\left[\operatorname{IEFPCM}_{(\mathrm{CH} 3 \mathrm{CN})} \mathrm{U} \omega \mathrm{B} 97 \mathrm{X}-\mathrm{D} / 6-311++\mathrm{G}(2 \mathrm{~d}, 2 \mathrm{p})\right]=-1961.73997276$

03

$\begin{array}{lrrr}\mathrm{O} & -0.41703900 & -1.39108700 & 1.42546900 \\ \mathrm{C} & -1.31355600 & -0.63338700 & 1.07524600 \\ \mathrm{C} & -2.69660200 & -1.11360700 & 0.95815500 \\ \mathrm{C} & -1.06295800 & 0.85438800 & 0.81905900 \\ \mathrm{C} & -3.74945100 & -0.33203100 & 0.64842400 \\ \mathrm{C} & -3.59809600 & 1.14752500 & 0.28694600 \\ \mathrm{C} & -5.14956700 & -0.90558300 & 0.70768900 \\ \mathrm{C} & -4.54911300 & 1.47397100 & -0.90657300 \\ \mathrm{C} & -5.98720700 & -0.54528200 & -0.52461900 \\ \mathrm{H} & -5.67241100 & -0.50171700 & 1.58773200 \\ \mathrm{H} & -5.10608200 & -1.99155900 & 0.82631900 \\ \mathrm{C} & -5.98684300 & 0.95839800 & -0.75467400 \\ \mathrm{H} & -4.57564300 & 2.55768500 & -1.06021300 \\ \mathrm{H} & -4.11850100 & 1.04102800 & -1.82037100 \\ \mathrm{H} & -5.59923600 & -1.07589500 & -1.39987800 \\ \mathrm{H} & -6.56340200 & 1.19725500 & -1.65586200\end{array}$




\begin{tabular}{|c|c|c|c|}
\hline $\mathrm{H}$ & -6.49618600 & 1.44914800 & 0.08427000 \\
\hline $\mathrm{H}$ & -2.83562100 & -2.16433000 & 1.20281600 \\
\hline $\mathrm{O}$ & -7.36295800 & -0.95249900 & -0.32591900 \\
\hline $\mathrm{C}$ & -7.67052200 & -2.24521400 & -0.60496000 \\
\hline $\mathrm{O}$ & -6.86374900 & -3.06416600 & -0.98605500 \\
\hline $\mathrm{C}$ & -9.14148800 & -2.50282100 & -0.37715700 \\
\hline $\mathrm{H}$ & -9.40656600 & -2.27958700 & 0.66151500 \\
\hline $\mathrm{H}$ & -9.74213700 & -1.84609600 & -1.01501200 \\
\hline $\mathrm{H}$ & -9.36607600 & -3.54589600 & -0.60177800 \\
\hline $\mathrm{C}$ & -0.40794000 & 3.20666200 & -0.93997800 \\
\hline $\mathrm{H}$ & -0.22272100 & 2.73690800 & -1.91476300 \\
\hline $\mathrm{H}$ & -0.27812400 & 4.28512800 & -1.09120400 \\
\hline $\mathrm{C}$ & 0.61156500 & 2.66901900 & 0.07578700 \\
\hline $\mathrm{C}$ & 0.34152300 & 1.13956200 & 0.26447800 \\
\hline $\mathrm{C}$ & 0.54572400 & 3.46042000 & 1.39906100 \\
\hline $\mathrm{H}$ & 1.20574600 & 3.04417600 & 2.16652100 \\
\hline $\mathrm{H}$ & 0.85547400 & 4.49600700 & 1.22692200 \\
\hline $\mathrm{H}$ & -0.46379400 & 3.48391900 & 1.81725600 \\
\hline $\mathrm{C}$ & 2.07849900 & 2.59388100 & -0.41448800 \\
\hline $\mathrm{C}$ & 1.60552700 & 0.62717900 & 0.94931700 \\
\hline $\mathrm{H}$ & 1.83082300 & -0.53084800 & 0.42434400 \\
\hline $\mathrm{C}$ & 2.74510100 & 1.55954000 & 0.51584300 \\
\hline $\mathrm{H}$ & 2.09626100 & 2.26617000 & -1.45727500 \\
\hline $\mathrm{H}$ & 3.18141800 & 2.06633400 & 1.38435200 \\
\hline $\mathrm{H}$ & 3.56907500 & 1.03872600 & 0.01745900 \\
\hline $\mathrm{O}$ & 2.80503700 & 3.83735600 & -0.32846600 \\
\hline $\mathrm{C}$ & 2.87479700 & 4.60928700 & -1.44328800 \\
\hline $\mathrm{O}$ & 2.31886400 & 4.34982900 & -2.48749900 \\
\hline $\mathrm{C}$ & 3.74786600 & 5.81680600 & -1.19392500 \\
\hline $\mathrm{H}$ & 3.72526900 & 6.46838900 & -2.06796400 \\
\hline $\mathrm{H}$ & 3.40368500 & 6.36103800 & -0.30883000 \\
\hline $\mathrm{H}$ & 4.77705200 & 5.49628500 & -0.99952200 \\
\hline $\mathrm{C}$ & -4.00569400 & 1.98979400 & 1.52512800 \\
\hline $\mathrm{H}$ & -5.00531400 & 1.72226200 & 1.87998800 \\
\hline $\mathrm{H}$ & -3.31481900 & 1.83560000 & 2.36039600 \\
\hline $\mathrm{H}$ & -4.02174700 & 3.05797100 & 1.28633500 \\
\hline $\mathrm{H}$ & 1.54901100 & 0.38440500 & 2.00989800 \\
\hline $\mathrm{H}$ & 0.35958000 & 0.71678400 & -0.75522400 \\
\hline $\mathrm{H}$ & -1.16504900 & 1.33763500 & 1.80301000 \\
\hline $\mathrm{H}$ & -1.99020700 & 0.87164500 & -1.09823600 \\
\hline $\mathrm{C}$ & -2.12415600 & 1.43438500 & -0.16040200 \\
\hline $\mathrm{C}$ & -1.85487400 & 2.92207300 & -0.49106400 \\
\hline $\mathrm{H}$ & -2.09000000 & 3.54498800 & 0.37945600 \\
\hline $\mathrm{H}$ & -2.53225500 & 3.24855100 & -1.28765800 \\
\hline $\mathrm{O}$ & 2.02238600 & -1.48724700 & -0.40504200 \\
\hline $\mathrm{C}$ & 3.29811200 & -1.84821800 & -0.5324690 \\
\hline
\end{tabular}




$\begin{array}{llll}\mathrm{C} & 4.26557200 & -1.72876700 & 0.56263500 \\ \mathrm{C} & 3.80566900 & -1.71477300 & 1.99482700 \\ \mathrm{C} & 4.69687900 & -1.19284700 & 2.94804400 \\ \mathrm{C} & 2.59913400 & -2.28414500 & 2.43296900 \\ \mathrm{C} & 4.37999600 & -1.21463800 & 4.30327600 \\ \mathrm{H} & 5.64082200 & -0.78565500 & 2.60064200 \\ \mathrm{C} & 2.28919600 & -2.31366200 & 3.79298500 \\ \mathrm{H} & 1.89344600 & -2.69072300 & 1.71859700 \\ \mathrm{C} & 3.17312300 & -1.77632800 & 4.73074300 \\ \mathrm{H} & 5.07606400 & -0.80079400 & 5.02858300 \\ \mathrm{H} & 1.35117700 & -2.75713100 & 4.11551100 \\ \mathrm{H} & 2.92610300 & -1.79839000 & 5.78924400 \\ \mathrm{C} & 3.63614400 & -2.42108700 & -1.83113100 \\ \mathrm{C} & 2.71016000 & -2.30361300 & -2.89642000 \\ \mathrm{C} & 4.83740500 & -3.13277300 & -2.06557100 \\ \mathrm{C} & 2.98060100 & -2.85881900 & -4.14106400 \\ \mathrm{H} & 1.78562500 & -1.76311400 & -2.72922600 \\ \mathrm{C} & 5.09833200 & -3.67796300 & -3.31698400 \\ \mathrm{H} & 5.55829600 & -3.23635400 & -1.26587200 \\ \mathrm{C} & 4.17685000 & -3.54784200 & -4.36172900 \\ \mathrm{H} & 2.25834400 & -2.74939900 & -4.94604000 \\ \mathrm{H} & 6.02911500 & -4.21545000 & -3.47860500 \\ \mathrm{H} & 4.38942300 & -3.97863200 & -5.33653100 \\ \mathrm{O} & 5.48568900 & -1.66886200 & 0.32178700\end{array}$




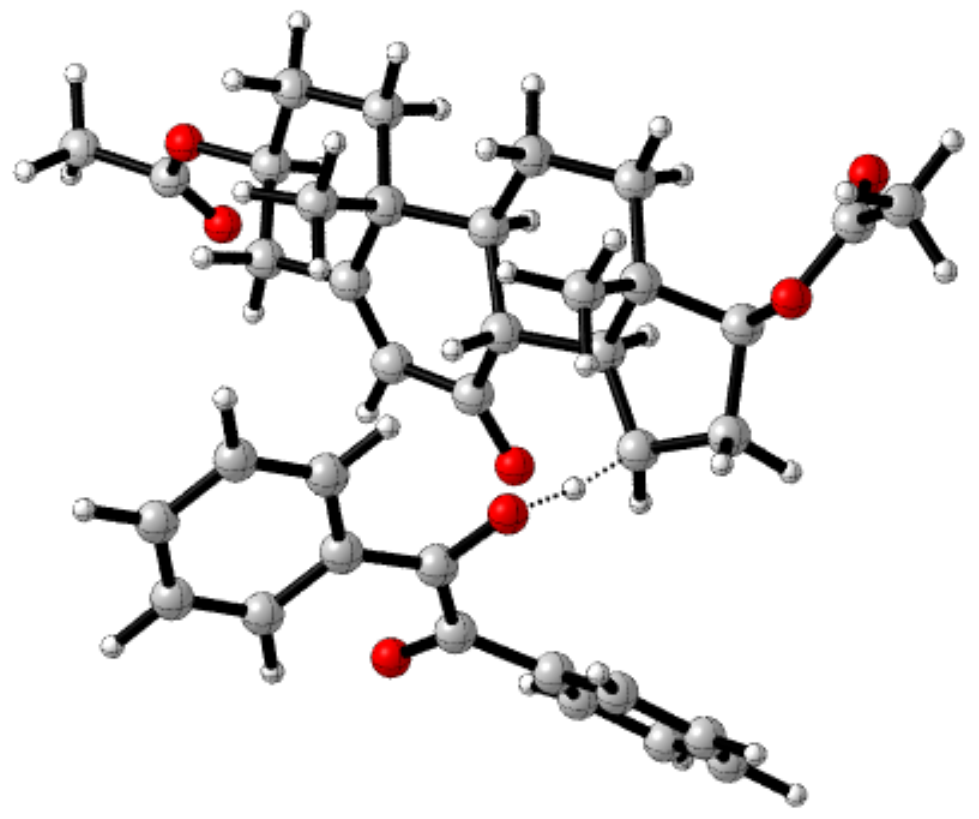

Figure S69. Transition state involving carbon C46 using trans benzil (top hydrogen).

Zero-point correction $=$

Thermal correction to Energy=

Thermal correction to Enthalpy=

Thermal correction to Gibbs Free Energy=

Sum of electronic and zero-point Energies $=$

Sum of electronic and thermal Energies=

Sum of electronic and thermal Enthalpies=

Sum of electronic and thermal Free Energies=
0.715450 (Hartree/Particle)

0.757025

0.757969

0.634696

$-1961.014080$

$-1960.972505$

$-1960.971561$

$-1961.094833$

Number of Imaginary Frequencies $=1$

E (Single Point Energy) $\left[\operatorname{IEFPCM}_{(\mathrm{CH} 3 \mathrm{CN})} \mathrm{U} \omega \mathrm{B} 97 \mathrm{X}-\mathrm{D} / 6-311++\mathrm{G}(2 \mathrm{~d}, 2 \mathrm{p})\right]=-1961.74463510$

03

$\begin{array}{lrrr}\mathrm{O} & -0.03062200 & -0.79119200 & -2.12985500 \\ \mathrm{C} & -0.74025000 & -0.25099300 & -1.28918100 \\ \mathrm{C} & -2.18124900 & -0.53277700 & -1.22145400 \\ \mathrm{C} & -0.19235000 & 0.72949400 & -0.25124800 \\ \mathrm{C} & -3.04323700 & 0.13729300 & -0.43246700 \\ \mathrm{C} & -2.61726000 & 1.32468900 & 0.43675000 \\ \mathrm{C} & -4.49394000 & -0.29080200 & -0.37020300 \\ \mathrm{C} & -3.68160100 & 2.45574600 & 0.28817800 \\ \mathrm{C} & -5.45786200 & 0.88915400 & -0.54402500 \\ \mathrm{H} & -4.70171900 & -0.74453900 & 0.61014200\end{array}$




\begin{tabular}{|c|c|c|c|}
\hline $\mathrm{H}$ & -4.69439900 & -1.05193100 & -1.12906400 \\
\hline $\mathrm{C}$ & -5.13923200 & 2.00015700 & 0.44576800 \\
\hline $\mathrm{H}$ & -3.47472400 & 3.24373300 & 1.01999400 \\
\hline $\mathrm{H}$ & -3.56404200 & 2.91347200 & -0.70421000 \\
\hline $\mathrm{H}$ & -5.40554700 & 1.25567000 & -1.57401500 \\
\hline $\mathrm{H}$ & -5.81327700 & 2.84894800 & 0.28131500 \\
\hline $\mathrm{H}$ & -5.33122200 & 1.63836800 & 1.46363100 \\
\hline $\mathrm{H}$ & -2.52127700 & -1.34653600 & -1.85768300 \\
\hline $\mathrm{O}$ & -6.81792600 & 0.45961100 & -0.28908300 \\
\hline $\mathrm{C}$ & -7.49137000 & -0.10660500 & -1.32261300 \\
\hline $\mathrm{O}$ & -7.01928300 & -0.27818000 & -2.42473000 \\
\hline $\mathrm{C}$ & -8.89265100 & -0.48260700 & -0.90110100 \\
\hline $\mathrm{H}$ & -9.42814500 & 0.40056400 & -0.53791200 \\
\hline $\mathrm{H}$ & -9.42226500 & -0.91728200 & -1.74934300 \\
\hline $\mathrm{H}$ & -8.85585800 & -1.20315600 & -0.07728200 \\
\hline $\mathrm{C}$ & 0.70156000 & 3.54633900 & 0.34141500 \\
\hline $\mathrm{H}$ & 0.63919900 & 3.98945300 & -0.66173900 \\
\hline $\mathrm{H}$ & 1.03444300 & 4.34883100 & 1.01034300 \\
\hline $\mathrm{C}$ & 1.71047700 & 2.38731200 & 0.32234000 \\
\hline $\mathrm{C}$ & 1.17207600 & 1.30559500 & -0.65249600 \\
\hline $\mathrm{C}$ & 1.96517800 & 1.86675700 & 1.75353500 \\
\hline $\mathrm{H}$ & 2.68760900 & 1.04833900 & 1.78133800 \\
\hline $\mathrm{H}$ & 2.35659300 & 2.67894300 & 2.37501200 \\
\hline $\mathrm{H}$ & 1.05024300 & 1.49985700 & 2.22550900 \\
\hline $\mathrm{C}$ & 3.06609700 & 2.67645700 & -0.37111000 \\
\hline $\mathrm{C}$ & 2.37078800 & 0.40871400 & -0.94237100 \\
\hline $\mathrm{H}$ & 2.42185800 & -0.51219300 & -0.04002900 \\
\hline $\mathrm{C}$ & 3.61249600 & 1.28648800 & -0.77442600 \\
\hline $\mathrm{H}$ & 2.89761800 & 3.30277500 & -1.25165300 \\
\hline $\mathrm{H}$ & 4.27743000 & 0.91299900 & 0.01425200 \\
\hline $\mathrm{H}$ & 4.21209600 & 1.32475200 & -1.69060900 \\
\hline $\mathrm{O}$ & 4.03737400 & 3.34121300 & 0.46391600 \\
\hline $\mathrm{C}$ & 4.14296500 & 4.69005600 & 0.36114000 \\
\hline $\mathrm{O}$ & 3.45428400 & 5.37530700 & -0.36247200 \\
\hline $\mathrm{C}$ & 5.23953500 & 5.20276500 & 1.26553400 \\
\hline $\mathrm{H}$ & 6.20038000 & 4.76529500 & 0.97468500 \\
\hline $\mathrm{H}$ & 5.29094800 & 6.28961600 & 1.19418400 \\
\hline $\mathrm{H}$ & 5.04667700 & 4.90416700 & 2.30104700 \\
\hline $\mathrm{C}$ & -2.56258100 & 0.85093700 & 1.91387900 \\
\hline $\mathrm{H}$ & -3.51103000 & 0.40336200 & 2.22418900 \\
\hline $\mathrm{H}$ & -1.78604200 & 0.09545800 & 2.07031000 \\
\hline $\mathrm{H}$ & -2.36627900 & 1.69046800 & 2.58854400 \\
\hline $\mathrm{H}$ & 2.30087800 & -0.20440200 & -1.83804400 \\
\hline $\mathrm{H}$ & 1.00027100 & 1.82503200 & -1.61622600 \\
\hline $\mathrm{H}$ & -0.08318500 & 0.16322700 & 0.68444800 \\
\hline $\mathrm{H}$ & -1.41868100 & 2.27259200 & -1.0670240 \\
\hline
\end{tabular}




$\begin{array}{lrrr}\mathrm{C} & -1.23350700 & 1.87300300 & -0.05650800 \\ \mathrm{C} & -0.69183400 & 3.05497300 & 0.78338400 \\ \mathrm{H} & -0.65292300 & 2.77956500 & 1.84270500 \\ \mathrm{H} & -1.39243100 & 3.89468000 & 0.71487000 \\ \mathrm{O} & 2.42199000 & -1.28022500 & 0.99688600 \\ \mathrm{C} & 1.77584500 & -2.43736100 & 0.81096100 \\ \mathrm{C} & 1.78241700 & -3.13037700 & -0.48167000 \\ \mathrm{C} & 2.88801800 & -2.86520000 & -1.46592900 \\ \mathrm{C} & 2.60255800 & -3.04086400 & -2.82866400 \\ \mathrm{C} & 4.20120300 & -2.55923600 & -1.07623700 \\ \mathrm{C} & 3.60362500 & -2.88578600 & -3.78464500 \\ \mathrm{H} & 1.58650300 & -3.28912900 & -3.11560900 \\ \mathrm{C} & 5.20586900 & -2.42228100 & -2.03467800 \\ \mathrm{H} & 4.43775400 & -2.43329500 & -0.02467100 \\ \mathrm{C} & 4.90895400 & -2.57833000 & -3.39042800 \\ \mathrm{H} & 3.36812700 & -3.00979500 & -4.83841200 \\ \mathrm{H} & 6.22247900 & -2.19845000 & -1.72156100 \\ \mathrm{H} & 5.69171600 & -2.46522400 & -4.13613000 \\ \mathrm{C} & 1.13374300 & -2.97122500 & 2.00361700 \\ \mathrm{C} & 1.02714100 & -2.15258600 & 3.15672700 \\ \mathrm{C} & 0.63544300 & -4.29573800 & 2.07877800 \\ \mathrm{C} & 0.43582400 & -2.63073300 & 4.31972100 \\ \mathrm{H} & 1.41336200 & -1.14044200 & 3.11845800 \\ \mathrm{C} & 0.04063400 & -4.75818600 & 3.24640500 \\ \mathrm{H} & 0.70780900 & -4.93876500 & 1.21220500 \\ \mathrm{C} & -0.06450500 & -3.93543100 & 4.37340600 \\ \mathrm{H} & 0.36152400 & -1.98277100 & 5.18950500 \\ \mathrm{H} & -0.34075800 & -5.77540500 & 3.28052600 \\ \mathrm{H} & -0.52907500 & -4.30765600 & 5.28255200 \\ \mathrm{O} & 0.91084400 & -3.96679200 & -0.76579100\end{array}$

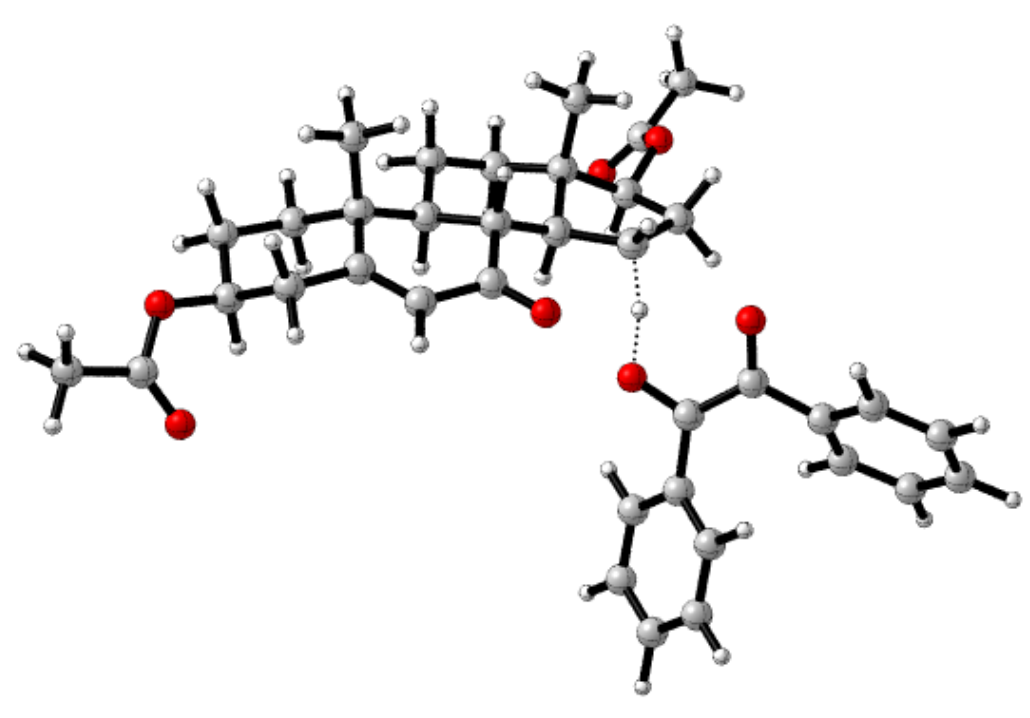


Figure S70. Transition state involving carbon C46 using cis benzil (bottom hydrogen).

\begin{tabular}{|c|c|c|c|}
\hline & 0.715651 (Hartree/Particle) \\
\hline \multirow{2}{*}{\multicolumn{3}{|c|}{ Thermal correction to Energy= }} & 0.757290 \\
\hline & & Thermal correction to Enthalpy= & 0.758234 \\
\hline \multirow{2}{*}{\multicolumn{3}{|c|}{$\begin{array}{l}\text { Thermal correction to Gibbs Free Energy= } \\
\text { Sum of electronic and zero-noint Energies= }\end{array}$}} & 0.633998 \\
\hline \multirow{2}{*}{\multicolumn{3}{|c|}{$\begin{array}{l}\text { Sum of electronic and zero-point Energies }= \\
\text { Sum of electronic and thermal Energies }=\end{array}$}} & -1961.015235 \\
\hline & & & -1960.973597 \\
\hline \multicolumn{3}{|c|}{ Sum of electronic and thermal Enthalpies $=$} & -1960.972652 \\
\hline \multicolumn{4}{|c|}{ Sum of electronic and thermal Free Energies $=$} \\
\hline \multicolumn{4}{|c|}{ Number of Imaginary Frequencies $=1$} \\
\hline \multicolumn{4}{|c|}{ E (Single Point Energy) $\left[\operatorname{IEFPCM}_{(\mathrm{CH} 3 \mathrm{CN})} \mathrm{U} \omega \mathrm{B} 97 \mathrm{X}-\mathrm{D} / 6-311++\mathrm{G}(2 \mathrm{~d}, 2 \mathrm{p})\right]=-1961.74049618$} \\
\hline \multicolumn{4}{|c|}{03} \\
\hline $\mathrm{O}$ & -0.20250600 & $-0.94430900 \quad-0.92$ & 100 \\
\hline $\mathrm{C}$ & 0.92782800 & $-0.48174700-0.831$ & 800 \\
\hline $\mathrm{C}$ & 2.09787300 & $-1.37101100-0.792$ & 300 \\
\hline $\mathrm{C}$ & 1.19498200 & $1.02538200-0.823$ & 200 \\
\hline $\mathrm{C}$ & 3.37546300 & $-0.94709500-0.745$ & 000 \\
\hline $\mathrm{C}$ & 3.74764900 & $0.53284500-0.627$ & 200 \\
\hline $\mathrm{C}$ & 4.50251600 & $-1.95195800-0.861$ & 600 \\
\hline $\mathrm{C}$ & 4.92882000 & $0.67489600 \quad 0.382$ & \\
\hline $\mathrm{C}$ & 5.59371000 & $-1.73592300 \quad 0.193$ & 300 \\
\hline $\mathrm{H}$ & 4.97828100 & $-1.85582300-1.84 \varepsilon$ & 600 \\
\hline $\mathrm{H}$ & 4.10806600 & $-2.96911600-0.78$ & 800 \\
\hline $\mathrm{C}$ & 6.09429500 & $-0.29977300 \quad 0.163$ & 700 \\
\hline $\mathrm{H}$ & 5.31255700 & $1.69966900 \quad 0.346$ & 000 \\
\hline $\mathrm{H}$ & 4.53558900 & $0.52557500 \quad 1.397$ & 500 \\
\hline $\mathrm{H}$ & 5.20417100 & $-1.99479100 \quad 1.182$ & 400 \\
\hline $\mathrm{H}$ & 6.85152300 & $-0.15347000 \quad 0.942$ & 500 \\
\hline $\mathrm{H}$ & 6.59054200 & $-0.11302500-0.79$ & 200 \\
\hline $\mathrm{H}$ & 1.86542600 & $-2.43119300-0.86$ & 700 \\
\hline $\mathrm{O}$ & 6.72701800 & $\begin{array}{ll}-2.59577100 & -0.08\end{array}$ & 800 \\
\hline $\mathrm{C}$ & 6.66202300 & $\begin{array}{ll}-3.87027900 & 0.379\end{array}$ & 900 \\
\hline $\mathrm{O}$ & 5.71542500 & $-4.32264500 \quad 0.985$ & 600 \\
\hline $\mathrm{C}$ & 7.92436500 & $-4.62550600 \quad 0.035$ & 200 \\
\hline $\mathrm{H}$ & 7.85308100 & $\begin{array}{ll}-5.64448900 & 0.417\end{array}$ & 600 \\
\hline $\mathrm{H}$ & 8.06775400 & $-4.64329000-1.04$ & 800 \\
\hline $\mathrm{H}$ & 8.79471800 & $-4.12370300 \quad 0.470$ & 300 \\
\hline $\mathrm{C}$ & 1.58477000 & $3.66675500 \quad 0.565$ & \\
\hline $\mathrm{H}$ & 1.41427000 & $3.40941900 \quad 1.618$ & 100 \\
\hline $\mathrm{H}$ & 1.82269500 & $4.73740300 \quad 0.546$ & 500 \\
\hline $\mathrm{C}$ & 0.30609000 & $3.37443200-0.234$ & \\
\hline $\mathrm{C}$ & 0.05162300 & $1.83154300-0.189$ & \\
\hline
\end{tabular}




$\begin{array}{lrrr}\mathrm{C} & 0.41302100 & 3.92589300 & -1.67170600 \\ \mathrm{H} & -0.46188000 & 3.67390000 & -2.27870700 \\ \mathrm{H} & 0.48994500 & 5.01752400 & -1.64501700 \\ \mathrm{H} & 1.29344200 & 3.54634500 & -2.19679200 \\ \mathrm{C} & -1.01941000 & 3.85284600 & 0.40898100 \\ \mathrm{C} & -1.39269100 & 1.69315100 & -0.66370400 \\ \mathrm{H} & -1.90988000 & 0.77011600 & 0.02382200 \\ \mathrm{C} & -2.10957100 & 2.98714700 & -0.25581300 \\ \mathrm{H} & -0.97727400 & 3.69015700 & 1.48912400 \\ \mathrm{H} & -2.51589200 & 3.50097300 & -1.13394200 \\ \mathrm{H} & -2.95421200 & 2.80070800 & 0.41288100 \\ \mathrm{O} & -1.32079900 & 5.24821900 & 0.18518400 \\ \mathrm{C} & -0.98069100 & 6.13515100 & 1.15250300 \\ \mathrm{O} & -0.39651600 & 5.84415700 & 2.17336700 \\ \mathrm{C} & -1.44263700 & 7.52494600 & 0.77852300 \\ \mathrm{H} & -1.04351300 & 7.80797800 & -0.20075300 \\ \mathrm{H} & -2.53502100 & 7.54672500 & 0.70300800 \\ \mathrm{H} & -1.11125900 & 8.23477500 & 1.53709900 \\ \mathrm{C} & 4.19537300 & 1.03032000 & -2.02768300 \\ \mathrm{H} & 4.99307900 & 0.40478300 & -2.43905300 \\ \mathrm{H} & 3.36756900 & 1.01012100 & -2.74387100 \\ \mathrm{H} & 4.57977300 & 2.05403000 & -1.97716600 \\ \mathrm{H} & -1.56148900 & 1.34949400 & -1.68240700 \\ \mathrm{H} & 0.05110600 & 1.57057300 & 0.88325300 \\ \mathrm{H} & 1.28908100 & 1.30837200 & -1.88386700 \\ \mathrm{H} & 2.37255400 & 0.98051500 & 0.95066800 \\ \mathrm{H} & -2.52408000 & 1.34355300 & -0.07870800 \\ \mathrm{C} & -2.78410500 & 2.86598100 & 0.02096200 \\ \mathrm{C} & 3.06243700 & 3.26108800 & -0.96266700 \\ \mathrm{H} & 3.64463700 & 3.04807400 & 0.67413400 \\ \mathrm{H} & -2.29318500 & -0.02218700 & 1.00677500 \\ \mathrm{O} & -3.23605100 & -0.85544400 & 0.62810900 \\ \mathrm{C} & -3.96792300 & -0.62737100 & -0.61470200 \\ \mathrm{C} & -4.75514900 & -1.72656300 & -1.26192900 \\ \mathrm{C} & -5.90545700 & -1.37436700 & -1.98560800 \\ \mathrm{C} & -4.33251400 & -3.06484600 & -1.26636700 \\ \mathrm{C} & -6.63967000 & -2.34382400 & -2.66462700 \\ \mathrm{H} & -6.20531600 & -0.33140800 & -2.00833800 \\ \mathrm{H} & -5.06028400 & -4.03148600 & -1.95961200 \\ \mathrm{H} & -3.42450800 & -3.34438100 & -0.74225700 \\ \mathrm{H} & -21971000 & -3.67666000 & -2.65241800 \\ \mathrm{H} & -2.7577800 & -2.05987900 & -3.21051100 \\ \mathrm{H} & -5.06284000 & -1.96287200 \\ \mathrm{H} & -4300 & -4.43288300 & -3.18772900 \\ \mathrm{H} & -1.90375200 & 1.62404200 \\ \mathrm{H} & -2.39528300 & 2.41611900\end{array}$




$\begin{array}{lrrr}\mathrm{C} & -4.82032500 & -2.37928800 & 1.87081800 \\ \mathrm{C} & -2.69276000 & -3.35549200 & 3.39585000 \\ \mathrm{H} & -1.45499600 & -2.02495900 & 2.23453400 \\ \mathrm{C} & -5.04700600 & -3.33857100 & 2.85326300 \\ \mathrm{H} & -5.65335200 & -1.98754100 & 1.29752300 \\ \mathrm{C} & -3.98673500 & -3.83357000 & 3.61843200 \\ \mathrm{H} & -1.86301200 & -3.73383800 & 3.98725700 \\ \mathrm{H} & -6.05862800 & -3.69426700 & 3.03000100 \\ \mathrm{H} & -4.16937000 & -4.58132900 & 4.38550500 \\ \mathrm{O} & -3.92356300 & 0.49049900 & -1.16943300\end{array}$

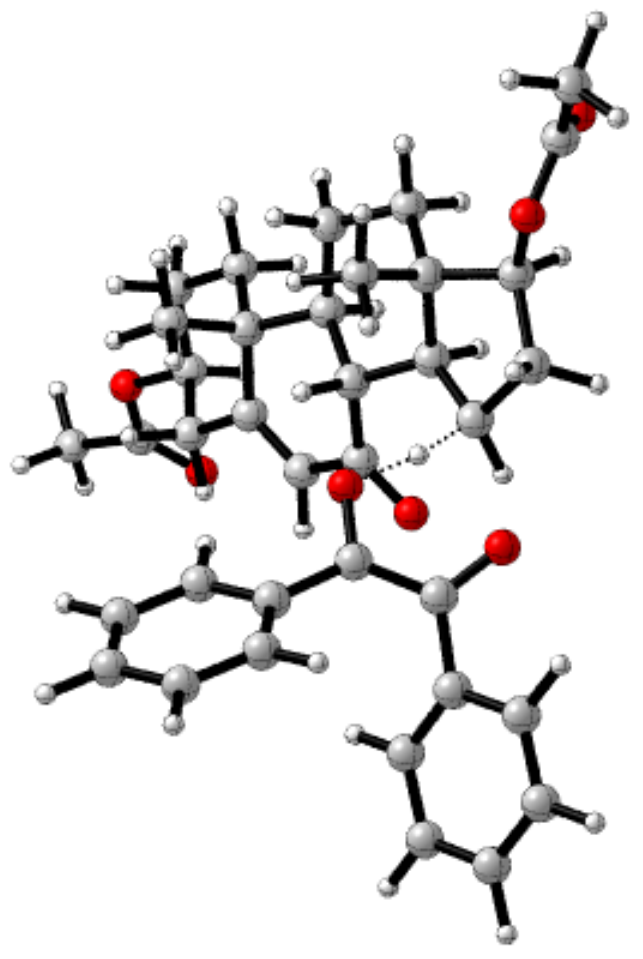

Figure S71. Transition state involving carbon C46 using cis benzil (top hydrogen).

Zero-point correction=

Thermal correction to Energy=

Thermal correction to Enthalpy=

Thermal correction to Gibbs Free Energy=

Sum of electronic and zero-point Energies=

Sum of electronic and thermal Energies=

Sum of electronic and thermal Enthalpies $=$

Sum of electronic and thermal Free Energies=
0.715513 (Hartree/Particle)

0.757170

0.758115

0.633947

$-1961.016848$

$-1960.975190$

$-1960.974246$

$-1961.098413$

Number of Imaginary Frequencies $=1$ 
E (Single Point Energy) IEFPCM $_{(\mathrm{CH} 3 \mathrm{CN})} \mathrm{U \omega B}$ B7X-D/6-311++G(2d,2p)] = -1961.74232521

03

$\begin{array}{lrrr}\mathrm{O} & -0.27478100 & -0.68025900 & -2.04131700 \\ \mathrm{C} & -1.13180300 & -0.38995500 & -1.21545200 \\ \mathrm{C} & -2.34805900 & -1.20776900 & -1.08174600 \\ \mathrm{C} & -0.99687100 & 0.79231700 & -0.25604800 \\ \mathrm{C} & -3.38530000 & -0.89926700 & -0.28023500 \\ \mathrm{C} & -3.43953100 & 0.39297400 & 0.53890200 \\ \mathrm{C} & -4.54603300 & -1.86299700 & -0.14875200 \\ \mathrm{C} & -4.86955800 & 1.00355300 & 0.40704100 \\ \mathrm{C} & -5.90305700 & -1.16792700 & -0.31154600 \\ \mathrm{H} & -4.53146900 & -2.32352100 & 0.85032100 \\ \mathrm{H} & -4.44986800 & -2.66989600 & -0.88021600 \\ \mathrm{C} & -6.02201400 & 0.01576200 & 0.63709900 \\ \mathrm{H} & -4.97185300 & 1.83760800 & 1.10900500 \\ \mathrm{H} & -4.97146400 & 1.43052800 & -0.60054900 \\ \mathrm{H} & -6.02901100 & -0.85060800 & -1.35149200 \\ \mathrm{H} & -6.98176000 & 0.52203300 & 0.48101600 \\ \mathrm{H} & -6.02505200 & -0.35287600 & 1.67059300 \\ \mathrm{H} & -2.35645700 & -2.11632100 & -1.68005900 \\ \mathrm{O} & -6.97501100 & -2.08868700 & 0.00976800 \\ \mathrm{C} & -7.38833100 & -2.92455700 & -0.97620400 \\ \mathrm{O} & -6.90458000 & -2.95276000 & -2.08631000 \\ \mathrm{C} & -8.52374500 & -3.79625500 & -0.49264900 \\ \mathrm{H} & -8.83908400 & -4.46088800 & -1.29749100 \\ \mathrm{H} & -8.20386600 & -4.38511400 & 0.37332600 \\ \mathrm{H} & -9.36551300 & -3.17442300 & -0.17069400 \\ \mathrm{C} & -1.29171000 & 3.74567500 & 0.26627700 \\ \mathrm{H} & -1.58841600 & 4.11018900 & -0.72647500 \\ \mathrm{H} & -1.27167300 & 4.62515100 & 0.92099100 \\ \mathrm{C} & 0.09660000 & 3.09466500 & 0.17102100 \\ \mathrm{C} & -0.01362800 & 1.85869100 & -0.76325400 \\ \mathrm{C} & 0.64142100 & 2.75460400 & 1.57512500 \\ \mathrm{H} & 1.60875600 & 2.24764400 & 1.53884100 \\ \mathrm{H} & 0.76837700 & 3.67530200 & 2.15380800 \\ \mathrm{H} & -0.03604000 & 2.10345900 & 2.13328700 \\ \mathrm{C} & 1.16203300 & 3.89519800 & -0.61985200 \\ \mathrm{C} & 1.43162700 & 1.52580700 & -1.13386300 \\ \mathrm{H} & 1.87320600 & 0.71909700 & -0.26630800 \\ \mathrm{C} & 2.20309900 & 2.84426500 & -1.06234600 \\ \mathrm{H} & 0.68824400 & 4.37365900 & -1.48167300 \\ \mathrm{H} & 3.02011800 & 2.79407300 & -0.33620700 \\ \mathrm{H} & 2.66659100 & 3.09292300 & -2.02311200 \\ \mathrm{O} & 1.82428800 & 4.92678900 & 0.14475700 \\ \mathrm{C} & 1.35822600 & 6.19460400 & 0.03778900 \\ & & & \end{array}$




\begin{tabular}{|c|c|c|c|}
\hline $\mathrm{O}$ & 0.39840900 & 6.51797100 & -0.62782200 \\
\hline $\mathrm{C}$ & 2.20974300 & 7.13911700 & 0.85493900 \\
\hline $\mathrm{H}$ & 1.79964400 & 8.14741900 & 0.78964300 \\
\hline $\mathrm{H}$ & 2.24076000 & 6.81388300 & 1.89984700 \\
\hline $\mathrm{H}$ & 3.23932000 & 7.13195600 & 0.48184500 \\
\hline $\mathrm{C}$ & -3.15836800 & 0.03840200 & 2.02346200 \\
\hline $\mathrm{H}$ & -3.82353400 & -0.75352000 & 2.37972600 \\
\hline $\mathrm{H}$ & -2.13144700 & -0.31383800 & 2.16422600 \\
\hline $\mathrm{H}$ & -3.31310600 & 0.90667000 & 2.67166000 \\
\hline $\mathrm{H}$ & 1.56512900 & 0.91578500 & -2.02422200 \\
\hline $\mathrm{H}$ & -0.44595700 & 2.23215300 & -1.71277000 \\
\hline $\mathrm{H}$ & -0.61472600 & 0.37455000 & 0.68620900 \\
\hline $\mathrm{H}$ & -2.76955400 & 1.69466300 & -1.02720300 \\
\hline $\mathrm{C}$ & -2.40194400 & 1.42580600 & -0.02338300 \\
\hline $\mathrm{C}$ & -2.33922500 & 2.74252200 & 0.78748900 \\
\hline $\mathrm{H}$ & -2.13027200 & 2.52453500 & 1.84033300 \\
\hline $\mathrm{H}$ & -3.32216800 & 3.22650600 & 0.76775700 \\
\hline $\mathrm{O}$ & 2.02429200 & -0.17320300 & 0.72078700 \\
\hline $\mathrm{C}$ & 3.01926600 & -1.01031500 & 0.50823900 \\
\hline $\mathrm{C}$ & 3.97220600 & -0.76748900 & -0.57278300 \\
\hline $\mathrm{C}$ & 4.86340900 & -1.85911800 & -1.08359200 \\
\hline $\mathrm{C}$ & 6.12253600 & -1.49569400 & -1.58714400 \\
\hline $\mathrm{C}$ & 4.45280300 & -3.19765100 & -1.18049100 \\
\hline $\mathrm{C}$ & 6.96810900 & -2.45491100 & -2.13907200 \\
\hline $\mathrm{H}$ & 6.41829800 & -0.45235100 & -1.54259800 \\
\hline $\mathrm{C}$ & 5.29427000 & -4.15371000 & -1.74811400 \\
\hline $\mathrm{H}$ & 3.46856800 & -3.48756300 & -0.82744500 \\
\hline $\mathrm{C}$ & 6.55649300 & -3.78788000 & -2.22029300 \\
\hline $\mathrm{H}$ & 7.94537500 & -2.16254800 & -2.51433800 \\
\hline $\mathrm{H}$ & 4.96043600 & -5.18491800 & -1.82690900 \\
\hline $\mathrm{H}$ & 7.21248700 & -4.53577600 & -2.65806100 \\
\hline $\mathrm{C}$ & 3.11427800 & -2.06928700 & 1.52788000 \\
\hline $\mathrm{C}$ & 1.93487100 & -2.56108100 & 2.12532900 \\
\hline $\mathrm{C}$ & 4.35418300 & -2.55564800 & 1.99069000 \\
\hline $\mathrm{C}$ & 1.99390700 & -3.53056200 & 3.12204800 \\
\hline $\mathrm{H}$ & 0.97704900 & -2.18324300 & 1.78415600 \\
\hline $\mathrm{C}$ & 4.40543900 & -3.52390700 & 2.98885400 \\
\hline $\mathrm{H}$ & 5.27511900 & -2.16448200 & 1.57299400 \\
\hline $\mathrm{C}$ & 3.22828400 & -4.01912100 & 3.55755000 \\
\hline $\mathrm{H}$ & 1.07357400 & -3.90778400 & 3.56032200 \\
\hline $\mathrm{H}$ & 5.37059200 & -3.88645800 & 3.33231000 \\
\hline $\mathrm{H}$ & 3.27380200 & -4.77426900 & 4.33752600 \\
\hline $\mathrm{O}$ & 4.03052000 & 0.35858100 & -1.1060410 \\
\hline
\end{tabular}


Table S7. Single point energies of all structures calculated at the $\operatorname{IEFPCM}_{(\mathrm{CH} 3 \mathrm{CN})} \mathrm{U \omega B} 97 \mathrm{X}-\mathrm{D} / 6$ $311++\mathrm{G}(2 \mathrm{~d}, 2 \mathrm{p})$ level of theory and thermal correction to Gibbs free energy and Gibbs free energy calculated at the UB3LYP/6-31G(d) level of theory. All energies are reported in Hartrees.

\begin{tabular}{|c|c|c|c|c|}
\hline Structure & 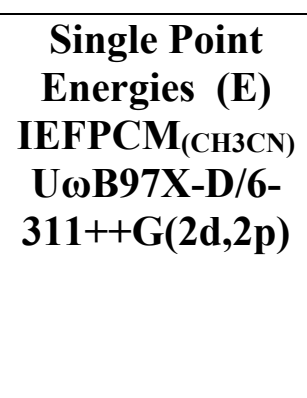 & $\begin{array}{c}\text { Thermal } \\
\text { Corrections } \\
\text { to Gibbs } \\
\text { Free } \\
\text { Energies } \\
\text { (G) } \\
\text { UB3LYP/6- } \\
\text { 31G(d) }\end{array}$ & $\begin{array}{c}\text { Gibbs Free } \\
\text { Energies } \\
\text { (G) } \\
\text { UB3LYP/6- } \\
\text { 31G(d) }\end{array}$ & $\begin{array}{c}\text { Gibbs Free Energies } \\
(\text { G) } \\
\text { IEFPCM }_{(\mathrm{CH} 3 \mathrm{CN})} \mathrm{U} \omega \mathrm{B} 9 \\
7 \mathrm{X}-\mathrm{D} / 6- \\
\text { 311++G(2d,2p)//UB3 } \\
\mathrm{LYP} / 6-31 \mathrm{G}(\mathrm{d})\end{array}$ \\
\hline $\begin{array}{l}\text { Benzoyl } \\
\text { Radical }\end{array}$ & -344.90102130 & 0.066518 & -344.854109 & -344.8345033 \\
\hline $\begin{array}{c}\text { Benzoyl } \\
\text { Peroxyradical }\end{array}$ & -495.28053982 & 0.071739 & -495.224414 & -495.2088008 \\
\hline $\begin{array}{l}\text { Benzoyl } \\
\text { Fluoride }\end{array}$ & -444.82826549 & 0.071155 & -444.762434 & -444.7571105 \\
\hline 6 & -1358.54736669 & 0.640134 & -1357.860158 & -1357.907233 \\
\hline 6 at $\mathrm{H}_{\mathrm{a}}$ Site & -1357.88465840 & 0.623902 & -1357.219055 & -1357.260756 \\
\hline 6 at $\mathrm{H}_{\mathrm{b}}$ Site & -1357.87742678 & 0.624998 & -1357.210094 & -1357.252429 \\
\hline 6 at $H_{c}$ Site & -1357.87748951 & 0.624725 & -1357.210817 & -1357.252765 \\
\hline 6 at $H_{d}$ Site & -1357.90074556 & 0.626039 & -1357.234387 & -1357.274707 \\
\hline 6 at $\mathrm{H}_{\mathrm{e}}$ Site & -1357.87909209 & 0.624456 & -1357.211611 & -1357.254636 \\
\hline 6 at $\mathrm{H}_{\mathrm{f}}$ Site & -1357.88175128 & 0.621246 & -1357.218462 & -1357.260505 \\
\hline 6 at $\mathrm{H}_{\mathrm{h}}$ Site & -1357.87864434 & 0.627047 & -1357.208555 & -1357.251597 \\
\hline
\end{tabular}

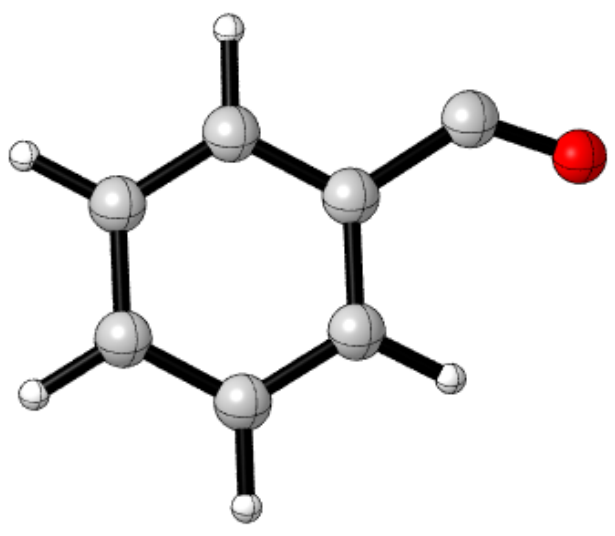

Figure S72. Optimized Structure of Benzoyl Radical.

Zero-point correction=

Thermal correction to Energy=

Thermal correction to Enthalpy=
0.097774 (Hartree/Particle)

0.104104

0.105049 
Thermal correction to Gibbs Free Energy=

Sum of electronic and zero-point Energies $=$

Sum of electronic and thermal Energies=

Sum of electronic and thermal Enthalpies=

Sum of electronic and thermal Free Energies=
0.066518

$-344.822852$

$-344.816522$

$-344.815578$

$-344.854109$

Number of Imaginary Frequencies $=0$

E (Single Point Energy) IEFPCM $_{(\mathrm{CH} 3 \mathrm{CN})} \mathrm{U \omega B}$ B7X-D/6-311++G(2d,2p)] = -344.90102130

02

$\begin{array}{lrrr}\mathrm{C} & 1.63213600 & -1.17327200 & 0.00000000 \\ \mathrm{C} & 1.33502600 & 0.18858400 & 0.00000000 \\ \mathrm{C} & 0.00000000 & 0.61237400 & 0.00000000 \\ \mathrm{C} & -1.04157600 & -0.33216900 & 0.00000000 \\ \mathrm{C} & -0.74015100 & -1.69028900 & 0.00000000 \\ \mathrm{C} & 0.59539700 & -2.10962300 & 0.00000000 \\ \mathrm{H} & 2.66633200 & -1.50537700 & 0.00000000 \\ \mathrm{H} & 2.12471900 & 0.93443800 & 0.00000000 \\ \mathrm{H} & -2.06969400 & 0.01742200 & 0.00000000 \\ \mathrm{H} & -1.54036700 & -2.42541900 & 0.00000000 \\ \mathrm{H} & 0.82685900 & -3.17146700 & 0.00000000 \\ \mathrm{C} & -0.29565200 & 2.06453700 & 0.00000000 \\ \mathrm{O} & -1.36486600 & 2.59869400 & 0.00000000\end{array}$

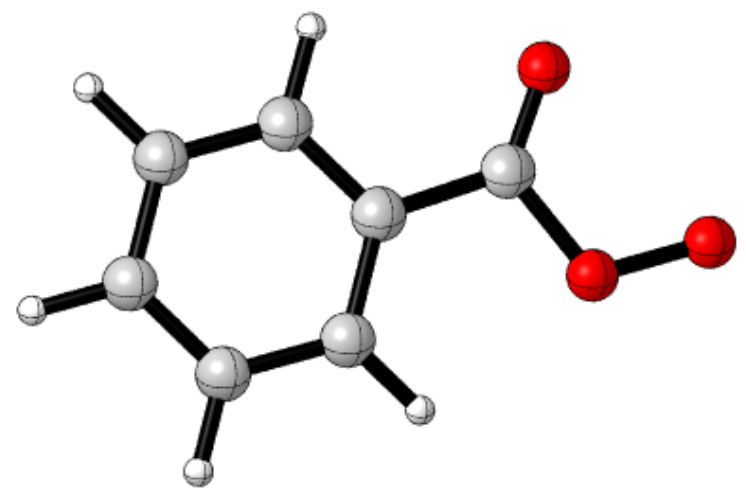

Figure S73. Optimized Structure of Benzoyl Peroxyradical.

Zero-point correction $=$

Thermal correction to Energy=

Thermal correction to Enthalpy=

Thermal correction to Gibbs Free Energy=

Sum of electronic and zero-point Energies=

Sum of electronic and thermal Energies=

Sum of electronic and thermal Enthalpies=

Sum of electronic and thermal Free Energies=
0.106295 (Hartree/Particle)

0.114495

0.115440

0.071739

$-495.189858$

$-495.181658$

$-495.180714$

$-495.224414$ 
Number of Imaginary Frequencies $=0$

E (Single Point Energy) [IEFPCM $\left(\mathrm{CH}_{3 \mathrm{CN})} \mathrm{U} \omega \mathrm{B} 97 \mathrm{X}-\mathrm{D} / 6-311++\mathrm{G}(2 \mathrm{~d}, 2 \mathrm{p})\right]=-495.28053982$

$\begin{array}{lrrr}02 & & & \\ \mathrm{C} & -2.04987200 & -1.37082600 & 0.00001500 \\ \mathrm{C} & -0.67099000 & -1.17206800 & -0.00004500 \\ \mathrm{C} & -0.15786300 & 0.13557100 & -0.00004900 \\ \mathrm{C} & -1.03335100 & 1.23389400 & -0.00000100 \\ \mathrm{C} & -2.40844600 & 1.02631500 & 0.00006200 \\ \mathrm{C} & -2.91745400 & -0.27604500 & 0.00007000 \\ \mathrm{H} & -2.44778200 & -2.38133900 & 0.00002100 \\ \mathrm{H} & 0.00288300 & -2.02065000 & -0.00008400 \\ \mathrm{H} & -0.61613600 & 2.23551500 & -0.00000900 \\ \mathrm{H} & -3.08441600 & 1.87637400 & 0.00010300 \\ \mathrm{H} & -3.99202600 & -0.43727700 & 0.00011900 \\ \mathrm{C} & 1.28884300 & 0.42955400 & -0.00008300 \\ \mathrm{O} & 1.82872100 & 1.49633800 & -0.00005100 \\ \mathrm{O} & 2.04524300 & -0.80477300 & -0.00027200 \\ \mathrm{O} & 3.35507000 & -0.60543900 & 0.00032600\end{array}$

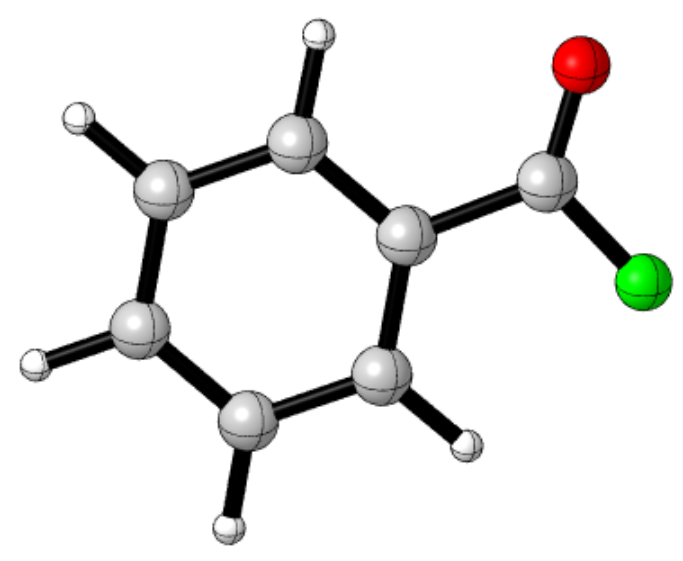

Figure S74. Optimized Structure of Benzoyl Fluoride.

Zero-point correction=

Thermal correction to Energy=

Thermal correction to Enthalpy=

Thermal correction to Gibbs Free Energy=

Sum of electronic and zero-point Energies $=$

Sum of electronic and thermal Energies $=$

Sum of electronic and thermal Enthalpies=

Sum of electronic and thermal Free Energies=
0.103112 (Hartree/Particle)

0.110049

0.110993

0.071155

$-444.730478$

$-444.723541$

$-444.722597$

$-444.762434$

Number of Imaginary Frequencies $=0$ 
E (Single Point Energy) $\left[\operatorname{IEFPCM}_{(\mathrm{CH} 3 \mathrm{CN})} \mathrm{U} \omega \mathrm{B} 97 \mathrm{X}-\mathrm{D} / 6-311++\mathrm{G}(2 \mathrm{~d}, 2 \mathrm{p})\right]=-444.82826549$

$\begin{array}{lrrr}01 & & & \\ \mathrm{C} & -0.88001600 & -2.01270900 & 0.00000000 \\ \mathrm{C} & -1.09573700 & -0.63630200 & 0.00000000 \\ \mathrm{C} & 0.00000000 & 0.23856000 & 0.00000000 \\ \mathrm{C} & 1.30783900 & -0.26955000 & 0.00000000 \\ \mathrm{C} & 1.51627500 & -1.64530000 & 0.00000000 \\ \mathrm{C} & 0.42287800 & -2.51688000 & 0.00000000 \\ \mathrm{H} & -1.72726800 & -2.69232100 & 0.00000000 \\ \mathrm{H} & -2.10285100 & -0.23548400 & 0.00000000 \\ \mathrm{H} & 2.14081400 & 0.42586800 & 0.00000000 \\ \mathrm{H} & 2.52811500 & -2.04009500 & 0.00000000 \\ \mathrm{H} & 0.58723700 & -3.59103800 & 0.00000000 \\ \mathrm{C} & -0.17520800 & 1.70501400 & 0.00000000 \\ \mathrm{O} & 0.67591200 & 2.54495800 & 0.00000000 \\ \mathrm{~F} & -1.48994800 & 2.06626700 & 0.00000000\end{array}$

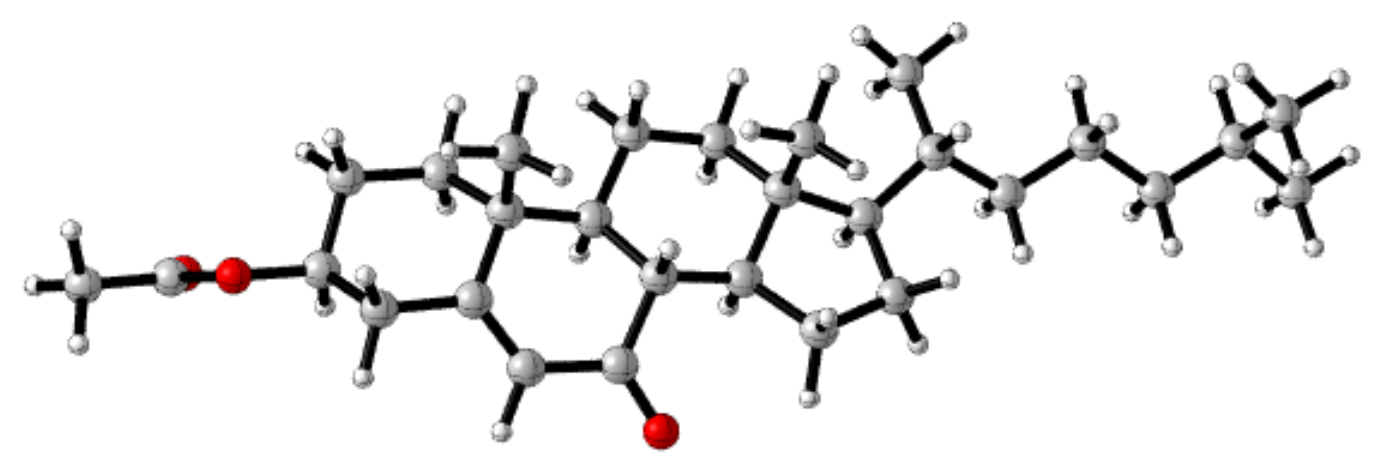

Figure S75. Optimized Structure of 6.

Zero-point correction=

Thermal correction to Energy=

Thermal correction to Enthalpy=

Thermal correction to Gibbs Free Energy=

Sum of electronic and zero-point Energies $=$

Sum of electronic and thermal Energies=

Sum of electronic and thermal Enthalpies $=$

Sum of electronic and thermal Free Energies=

Number of Imaginary Frequencies $=0$

E (Single Point Energy) IEFPCM $\left._{(\mathrm{CH} 3 \mathrm{CN})} \mathrm{U} \omega \mathrm{B} 97 \mathrm{X}-\mathrm{D} / 6-311++\mathrm{G}(2 \mathrm{~d}, 2 \mathrm{p})\right]=-1358.54736669$

01

$\begin{array}{llll}\text { C } & 5.96037000 & -0.14555000 & -0.34592200\end{array}$
0.707141 (Hartree/Particle)

0.740856

0.741801

0.640134

$-1357.793151$

$-1357.759435$

$-1357.758491$

$-1357.860158$ 


\begin{tabular}{|c|c|c|c|}
\hline $\mathrm{C}$ & 5.31662600 & 1.02011900 & 0.40617200 \\
\hline $\mathrm{C}$ & 3.80586700 & 1.00340900 & 0.30468500 \\
\hline $\mathrm{C}$ & 3.08642800 & -0.31059300 & 0.61786600 \\
\hline $\mathrm{C}$ & 3.84504800 & -1.47441700 & -0.09256500 \\
\hline $\mathrm{C}$ & 5.36768200 & -1.47542500 & 0.10657200 \\
\hline $\mathrm{H}$ & 5.61758400 & 0.94076100 & 1.46123000 \\
\hline $\mathrm{H}$ & 5.70938200 & 1.97338200 & 0.03817600 \\
\hline $\mathrm{H}$ & 5.82822800 & -0.02998200 & -1.42624100 \\
\hline $\mathrm{H}$ & 3.44104900 & -2.43224400 & 0.25164600 \\
\hline $\mathrm{H}$ & 3.63561000 & -1.41822900 & -1.16994700 \\
\hline $\mathrm{H}$ & 5.62860400 & -1.64043800 & 1.15985700 \\
\hline $\mathrm{H}$ & 5.81496400 & -2.29117700 & -0.47066400 \\
\hline $\mathrm{C}$ & 1.61998200 & -0.25577100 & 0.06558100 \\
\hline $\mathrm{H}$ & 1.72117000 & -0.22212500 & -1.03149800 \\
\hline $\mathrm{C}$ & 3.14437200 & 2.12647900 & -0.03109800 \\
\hline $\mathrm{H}$ & 3.68356700 & 3.03765700 & -0.28241200 \\
\hline $\mathrm{C}$ & 1.67622900 & 2.24984200 & -0.07510800 \\
\hline $\mathrm{C}$ & 0.87410300 & 1.05652800 & 0.45082900 \\
\hline $\mathrm{H}$ & 0.86880800 & 1.16355700 & 1.54736900 \\
\hline $\mathrm{O}$ & 7.38029500 & -0.08298400 & -0.05905200 \\
\hline $\mathrm{C}$ & 8.21864400 & -0.63346600 & -0.97231600 \\
\hline $\mathrm{O}$ & 7.84978400 & -1.17412500 & -1.99171000 \\
\hline $\mathrm{C}$ & 9.65699000 & -0.47138800 & -0.53830300 \\
\hline $\mathrm{H}$ & 10.31379500 & -0.92129500 & -1.28341800 \\
\hline $\mathrm{H}$ & 9.89794800 & 0.59025700 & -0.42101600 \\
\hline $\mathrm{H}$ & 9.81308900 & -0.95024200 & 0.43397300 \\
\hline $\mathrm{C}$ & 3.12199200 & -0.52826700 & 2.15412700 \\
\hline $\mathrm{H}$ & 2.53719800 & 0.23232400 & 2.68136900 \\
\hline $\mathrm{H}$ & 2.72330700 & -1.51205000 & 2.42126700 \\
\hline $\mathrm{H}$ & 4.14320400 & -0.47701200 & 2.54304600 \\
\hline $\mathrm{C}$ & 0.79093100 & -1.51717900 & 0.38936900 \\
\hline $\mathrm{H}$ & 1.27671500 & -2.39533400 & -0.05148400 \\
\hline $\mathrm{H}$ & 0.77626000 & -1.69106400 & 1.47124700 \\
\hline $\mathrm{C}$ & -0.57563600 & 1.03489500 & -0.06132500 \\
\hline $\mathrm{H}$ & -0.50831300 & 0.95595100 & -1.15893600 \\
\hline $\mathrm{C}$ & -0.66041700 & -1.45451500 & -0.12321600 \\
\hline $\mathrm{H}$ & -0.65823500 & -1.42994700 & -1.22293200 \\
\hline $\mathrm{H}$ & -1.16728500 & -2.38105400 & 0.16480300 \\
\hline $\mathrm{C}$ & -1.39769200 & -0.20744300 & 0.39986300 \\
\hline $\mathrm{C}$ & -2.77856700 & 0.11780900 & -0.26839300 \\
\hline $\mathrm{H}$ & -2.64828500 & -0.07270900 & -1.34586400 \\
\hline $\mathrm{C}$ & -2.91978900 & 1.66404800 & -0.06436500 \\
\hline $\mathrm{H}$ & -3.61173700 & 1.87957900 & 0.75930400 \\
\hline $\mathrm{H}$ & -3.34686400 & 2.13638300 & -0.95462400 \\
\hline $\mathrm{C}$ & -1.55541300 & -0.27188600 & 1.93648000 \\
\hline $\mathrm{H}$ & -2.13587500 & 0.57107900 & 2.32398800 \\
\hline
\end{tabular}




$\begin{array}{lrrr}\mathrm{H} & -2.07674700 & -1.18892200 & 2.23299400 \\ \mathrm{H} & -0.59447600 & -0.26680300 & 2.45974000 \\ \mathrm{C} & -4.04881100 & -0.65634700 & 0.17252500 \\ \mathrm{H} & -4.23554900 & -0.41933600 & 1.23149300 \\ \mathrm{C} & -5.27780400 & -0.15906400 & -0.62867800 \\ \mathrm{H} & -5.29078100 & 0.93732000 & -0.62656400 \\ \mathrm{H} & -5.15953900 & -0.45772300 & -1.68204900 \\ \mathrm{C} & -6.64253100 & -0.64282700 & -0.11274500 \\ \mathrm{H} & -6.72167600 & -0.40400300 & 0.95686200 \\ \mathrm{H} & -6.71876700 & -1.73575900 & -0.18985900 \\ \mathrm{C} & -7.81546900 & -0.01325200 & -0.87914200 \\ \mathrm{H} & -7.78882100 & 1.08039900 & -0.75339700 \\ \mathrm{H} & -7.66891300 & -0.19625000 & -1.95396200 \\ \mathrm{C} & -9.21433600 & -0.52730900 & -0.48375600 \\ \mathrm{H} & -9.20775500 & -1.62373400 & -0.58903400 \\ \mathrm{O} & 1.16103700 & 3.28004700 & -0.49115900 \\ \mathrm{C} & -3.91119200 & -2.18323000 & 0.04654500 \\ \mathrm{H} & -3.15889800 & -2.58796700 & 0.72864200 \\ \mathrm{H} & -4.85466000 & -2.68724900 & 0.27975000 \\ \mathrm{C} & -9.57374100 & -0.19672800 & 0.97307000 \\ \mathrm{H} & -8.87257000 & -0.64712000 & 1.68374900 \\ \mathrm{H} & -9.56694900 & 0.88893200 & 1.13910800 \\ \mathrm{H} & -10.57686800 & -0.56317400 & 1.22205000 \\ \mathrm{C} & -10.28146500 & 0.02763700 & -1.43936000 \\ \mathrm{H} & -10.06327200 & -0.23804200 & -2.48072500 \\ \mathrm{H} & -11.27699500 & -0.36174700 & -1.19468500 \\ \mathrm{H} & -10.32998100 & 1.12293200 & -1.37896800 \\ \mathrm{C} & -1.50388200 & 2.22303000 & 0.23665700 \\ \mathrm{H} & -1.41348000 & 2.52877100 & 1.28662300 \\ \mathrm{H} & -1.25046500 & 3.09521200 & -0.36470300 \\ \mathrm{H} & -3.62678200 & -2.47009200 & -0.97423700 \\ & & & \\ & & & \\ \mathrm{H} & & & \end{array}$

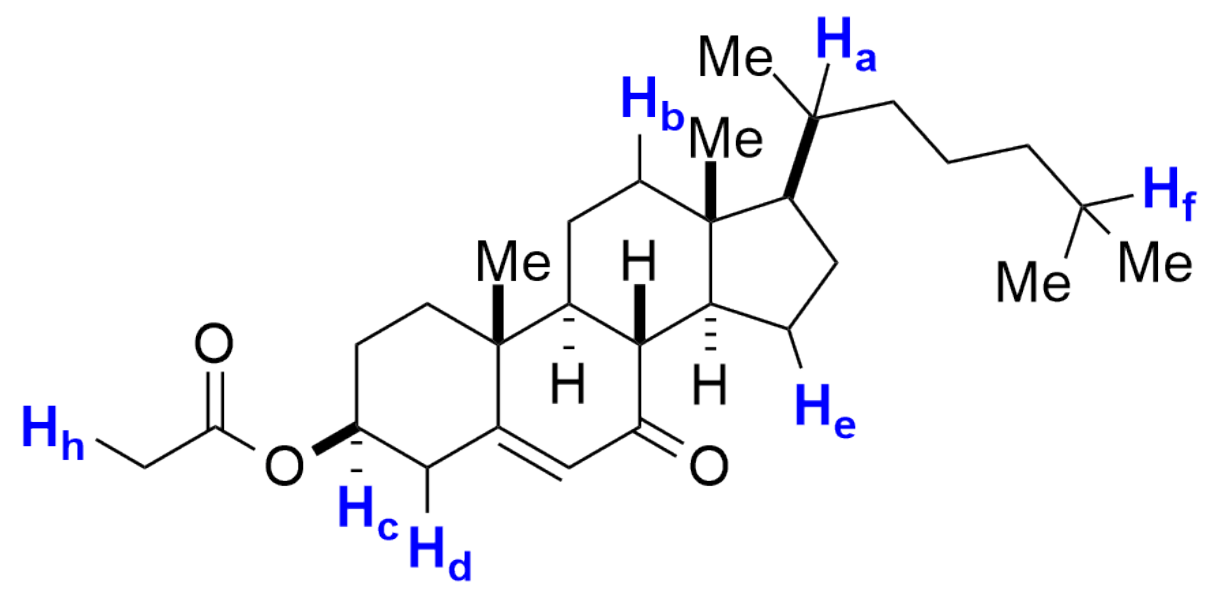

Figure S76. Depiction of 6 with pertinent hydrogen atoms labelled. 


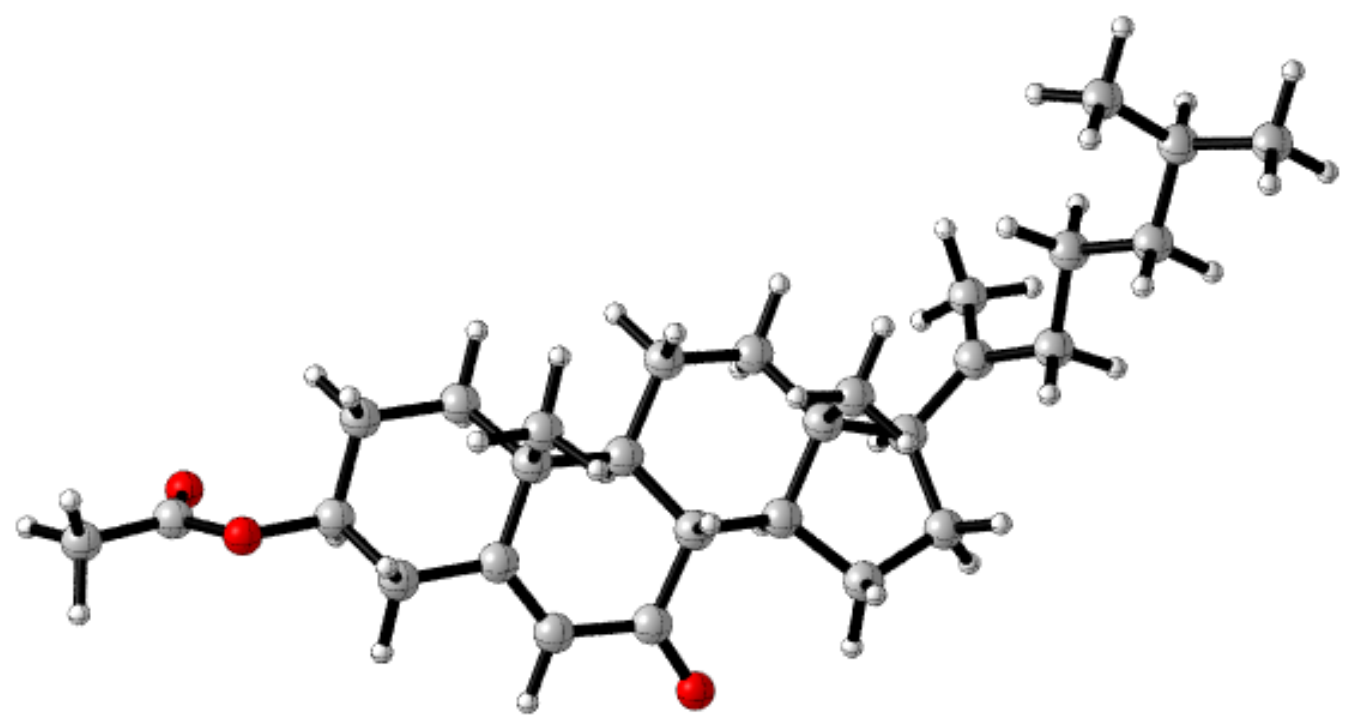

Figure S77. Optimized Structure of Steroid Radical at $\mathrm{H}_{\mathrm{a}}$ Site.

Zero-point correction $=$

Thermal correction to Energy=

Thermal correction to Enthalpy=

Thermal correction to Gibbs Free Energy=

Sum of electronic and zero-point Energies=

Sum of electronic and thermal Energies $=$

Sum of electronic and thermal Enthalpies=

Sum of electronic and thermal Free Energies=
0.692646 (Hartree/Particle)

0.726735

0.727679

0.623902

$-1357.150311$

$-1357.116221$

$-1357.115277$

$-1357.219055$

Number of Imaginary Frequencies $=0$

E (Single Point Energy) [IEFPCM $\left(\mathrm{CH}_{3 \mathrm{CN})} \mathrm{U} \omega \mathrm{B} 97 \mathrm{X}-\mathrm{D} / 6-311++\mathrm{G}(2 \mathrm{~d}, 2 \mathrm{p})\right]=-1357.88465840$

02

$\begin{array}{lrrr}\mathrm{C} & -5.58686000 & -0.52274800 & 0.02348600 \\ \mathrm{C} & -4.93595600 & 0.22554100 & -1.14052600 \\ \mathrm{C} & -3.49837100 & 0.60238500 & -0.84927400 \\ \mathrm{C} & -2.56979000 & -0.49095700 & -0.31489600 \\ \mathrm{C} & -3.32356500 & -1.28654900 & 0.79580600 \\ \mathrm{C} & -4.74708700 & -1.72910700 & 0.42776100 \\ \mathrm{H} & -4.97420300 & -0.43309600 & -2.02077800 \\ \mathrm{H} & -5.51703800 & 1.11880600 & -1.39112800 \\ \mathrm{H} & -5.72101500 & 0.14005200 & 0.88409600 \\ \mathrm{H} & -2.73687000 & -2.16992400 & 1.06854800 \\ \mathrm{H} & -3.37692200 & -0.65901400 & 1.69646300 \\ \mathrm{H} & -4.73577600 & -2.44794000 & -0.40163000 \\ \mathrm{H} & -5.21133900 & -2.22844200 & 1.28439100 \\ \mathrm{C} & -1.29057000 & 0.16186700 & 0.31510400\end{array}$




\begin{tabular}{|c|c|c|c|}
\hline $\mathrm{H}$ & -1.65043400 & 0.71742400 & 1.19647700 \\
\hline $\mathrm{C}$ & -3.07458900 & 1.85759700 & -1.08768200 \\
\hline $\mathrm{H}$ & -3.76458000 & 2.62208500 & -1.43926800 \\
\hline $\mathrm{C}$ & -1.68139100 & 2.31165200 & -0.93308600 \\
\hline $\mathrm{C}$ & -0.64061500 & 1.23774000 & -0.60900600 \\
\hline $\mathrm{H}$ & -0.37681900 & 0.77856500 & -1.57510300 \\
\hline $\mathrm{O}$ & -6.89983800 & -0.93146000 & -0.43624000 \\
\hline $\mathrm{C}$ & -7.85024400 & -1.13026900 & 0.51094300 \\
\hline $\mathrm{O}$ & -7.66057900 & -0.99638700 & 1.69991200 \\
\hline $\mathrm{C}$ & -9.15956500 & -1.53314700 & -0.12687100 \\
\hline $\mathrm{H}$ & -9.90872400 & -1.68934000 & 0.64989900 \\
\hline $\mathrm{H}$ & -9.49677300 & -0.75489700 & -0.81933800 \\
\hline $\mathrm{H}$ & -9.02818600 & -2.45176800 & -0.70814300 \\
\hline $\mathrm{C}$ & -2.21924600 & -1.44325100 & -1.48919900 \\
\hline $\mathrm{H}$ & -1.62932800 & -0.93328500 & -2.25751300 \\
\hline $\mathrm{H}$ & -1.64797700 & -2.30808100 & -1.13724800 \\
\hline $\mathrm{H}$ & -3.12037800 & -1.82758900 & -1.97612900 \\
\hline $\mathrm{C}$ & -0.26743500 & -0.87321200 & 0.84035800 \\
\hline $\mathrm{H}$ & -0.74073600 & -1.49141900 & 1.61168800 \\
\hline $\mathrm{H}$ & 0.01972500 & -1.55978200 & 0.03627100 \\
\hline $\mathrm{C}$ & 0.62862200 & 1.81180700 & 0.04178400 \\
\hline $\mathrm{H}$ & 0.29970200 & 2.26835600 & 0.98967100 \\
\hline $\mathrm{C}$ & 1.00430700 & -0.23525200 & 1.43151400 \\
\hline $\mathrm{H}$ & 0.74165500 & 0.32156800 & 2.34310300 \\
\hline $\mathrm{H}$ & 1.69981700 & -1.02814900 & 1.73784200 \\
\hline $\mathrm{C}$ & 1.66333600 & 0.72400400 & 0.43242500 \\
\hline $\mathrm{C}$ & 2.79296300 & 1.63926800 & 1.03869200 \\
\hline $\mathrm{H}$ & 2.35231400 & 2.03386000 & 1.96653200 \\
\hline $\mathrm{C}$ & 2.85164400 & 2.83228600 & 0.02767100 \\
\hline $\mathrm{H}$ & 3.66296400 & 2.70365400 & -0.69513300 \\
\hline $\mathrm{H}$ & 3.06356900 & 3.76479000 & 0.56166300 \\
\hline $\mathrm{C}$ & 2.20213800 & -0.05707900 & -0.78451100 \\
\hline $\mathrm{H}$ & 2.70642500 & 0.59433700 & -1.50435300 \\
\hline $\mathrm{H}$ & 2.92773500 & -0.80923500 & -0.45560500 \\
\hline $\mathrm{H}$ & 1.41329100 & -0.58474000 & -1.32933200 \\
\hline $\mathrm{C}$ & 4.10113500 & 1.00431600 & 1.42776700 \\
\hline $\mathrm{C}$ & 5.24498900 & 0.80679600 & 0.46790400 \\
\hline $\mathrm{H}$ & 4.93106100 & 1.02039700 & -0.56002500 \\
\hline $\mathrm{H}$ & 6.04180500 & 1.54262200 & 0.69130900 \\
\hline $\mathrm{C}$ & 5.89631100 & -0.59439000 & 0.49755400 \\
\hline $\mathrm{H}$ & 5.12659700 & -1.35091100 & 0.29660800 \\
\hline $\mathrm{H}$ & 6.27593000 & -0.80340900 & 1.50701800 \\
\hline $\mathrm{C}$ & 7.04683900 & -0.72671600 & -0.50972600 \\
\hline $\mathrm{H}$ & 6.65760300 & -0.58358700 & -1.52992500 \\
\hline $\mathrm{H}$ & 7.75567900 & 0.09727200 & -0.33963200 \\
\hline $\mathrm{C}$ & 7.82354800 & -2.05750300 & -0.45376500 \\
\hline
\end{tabular}




$\begin{array}{lrrr}\mathrm{O} & -1.39918700 & 3.49260000 & -1.09384600 \\ \mathrm{C} & 4.34048900 & 0.63475800 & 2.86473000 \\ \mathrm{H} & 3.57856500 & 1.06035600 & 3.52733800 \\ \mathrm{H} & 4.33834600 & -0.45510900 & 3.03469600 \\ \mathrm{H} & 5.32366400 & 0.98787300 & 3.21622000 \\ \mathrm{C} & 6.94707300 & -3.26741900 & -0.81248200 \\ \mathrm{H} & 6.10031300 & -3.38112100 & -0.12716800 \\ \mathrm{H} & 6.54368200 & -3.16842000 & -1.82923600 \\ \mathrm{H} & 7.52813400 & -4.19668500 & -0.77614600 \\ \mathrm{C} & 9.05774600 & -1.99864500 & -1.36660200 \\ \mathrm{H} & 9.71721300 & -1.16569100 & -1.09480600 \\ \mathrm{H} & 9.64288600 & -2.92409700 & -1.30592800 \\ \mathrm{H} & 8.76317800 & -1.86086400 & -2.41537500 \\ \mathrm{C} & 1.47002700 & 2.87688900 & -0.69058900 \\ \mathrm{H} & 0.99852300 & 3.85825500 & -0.64775100 \\ \mathrm{H} & 1.57067900 & 2.62668500 & -1.75391900 \\ \mathrm{H} & 8.17756300 & -2.19236700 & 0.58046500\end{array}$

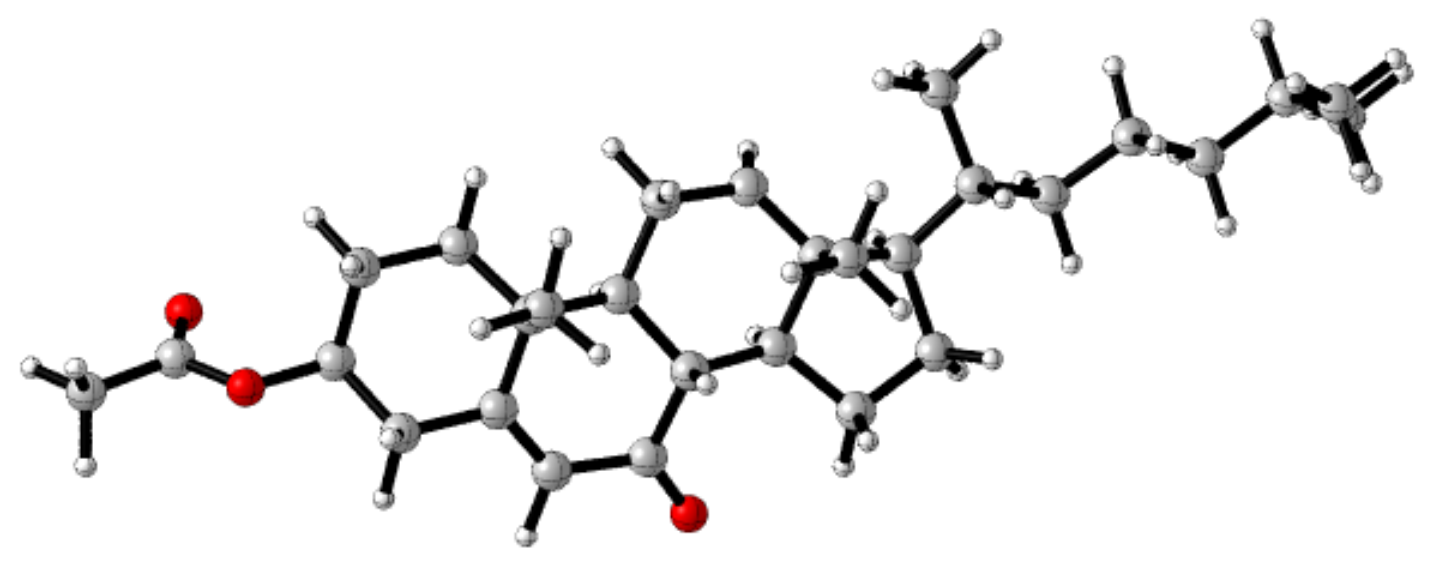

Figure S78. Optimized Structure of Steroid Radical at $\mathrm{H}_{\mathrm{b}}$ Site.

Zero-point correction $=$

Thermal correction to Energy=

Thermal correction to Enthalpy=

Thermal correction to Gibbs Free Energy=

Sum of electronic and zero-point Energies $=$

Sum of electronic and thermal Energies=

Sum of electronic and thermal Enthalpies=

Sum of electronic and thermal Free Energies=
0.692645 (Hartree/Particle)

0.726562

0.727506

0.624998

$-1357.142448$

$-1357.108531$

$-1357.107586$

$-1357.210094$

Number of Imaginary Frequencies $=0$

E (Single Point Energy) $\left[\operatorname{IEFPCM}_{(\mathrm{CH} 3 \mathrm{CN})} \mathrm{U} \omega \mathrm{B} 97 \mathrm{X}-\mathrm{D} / 6-311++\mathrm{G}(2 \mathrm{~d}, 2 \mathrm{p})\right]=-1357.87742678$ 


\begin{tabular}{|c|c|c|c|}
\hline $\mathrm{C}$ & 5.94386500 & -0.21983500 & -0.32219600 \\
\hline $\mathrm{C}$ & 5.32113300 & 0.97603100 & 0.39982300 \\
\hline $\mathrm{C}$ & 3.81143500 & 0.99357500 & 0.28476100 \\
\hline $\mathrm{C}$ & 3.05860700 & -0.29833600 & 0.61443700 \\
\hline $\mathrm{C}$ & 3.79624700 & -1.49431300 & -0.06312000 \\
\hline $\mathrm{C}$ & 5.31634900 & -1.52624900 & 0.15160300 \\
\hline $\mathrm{H}$ & 5.61013700 & 0.91109100 & 1.45923200 \\
\hline $\mathrm{H}$ & 5.74016800 & 1.91194000 & 0.01660400 \\
\hline $\mathrm{H}$ & 5.82410800 & -0.12353900 & -1.40584500 \\
\hline $\mathrm{H}$ & 3.36549400 & -2.43436400 & 0.29767200 \\
\hline $\mathrm{H}$ & 3.59877300 & -1.45656100 & -1.14354000 \\
\hline $\mathrm{H}$ & 5.56329600 & -1.67541900 & 1.21060000 \\
\hline $\mathrm{H}$ & 5.75020400 & -2.36371000 & -0.40434400 \\
\hline $\mathrm{C}$ & 1.60391800 & -0.21724900 & 0.03877900 \\
\hline $\mathrm{H}$ & 1.71972000 & -0.19694600 & -1.05595400 \\
\hline $\mathrm{C}$ & 3.17896500 & 2.12635800 & -0.07446500 \\
\hline $\mathrm{H}$ & 3.74135700 & 3.02077500 & -0.33479900 \\
\hline $\mathrm{C}$ & 1.71429400 & 2.28298500 & -0.13148600 \\
\hline $\mathrm{C}$ & 0.88021700 & 1.11496800 & 0.40292900 \\
\hline $\mathrm{H}$ & 0.86956200 & 1.23732000 & 1.49754200 \\
\hline $\mathrm{O}$ & 7.36197800 & -0.18425000 & -0.02312400 \\
\hline $\mathrm{C}$ & 8.19560000 & -0.77168500 & -0.91781800 \\
\hline $\mathrm{O}$ & 7.82313300 & -1.32333100 & -1.92993200 \\
\hline $\mathrm{C}$ & 9.63330200 & -0.63457400 & -0.47345400 \\
\hline $\mathrm{H}$ & 10.28616000 & -1.11289200 & -1.20419700 \\
\hline $\mathrm{H}$ & 9.89759000 & 0.42323100 & -0.37301300 \\
\hline $\mathrm{H}$ & 9.76967900 & -1.09918700 & 0.50865200 \\
\hline $\mathrm{C}$ & 3.06967200 & -0.48548400 & 2.15486500 \\
\hline $\mathrm{H}$ & 2.50365000 & 0.30326400 & 2.66055200 \\
\hline $\mathrm{H}$ & 2.63834800 & -1.45101600 & 2.43759700 \\
\hline $\mathrm{H}$ & 4.08798600 & -0.45795500 & 2.55379100 \\
\hline $\mathrm{C}$ & 0.73303400 & -1.47704200 & 0.35490500 \\
\hline $\mathrm{H}$ & 1.18828100 & -2.35632200 & -0.11214800 \\
\hline $\mathrm{H}$ & 0.74894100 & -1.65777000 & 1.43847300 \\
\hline $\mathrm{C}$ & -0.56536100 & 1.12612000 & -0.11979800 \\
\hline $\mathrm{H}$ & -0.49720600 & 1.01967700 & -1.21386900 \\
\hline $\mathrm{C}$ & -0.68317000 & -1.31889900 & -0.11310400 \\
\hline $\mathrm{C}$ & -1.41219200 & -0.10346500 & 0.37275700 \\
\hline $\mathrm{C}$ & -2.78256000 & 0.22737700 & -0.29700100 \\
\hline $\mathrm{H}$ & -2.65115100 & 0.03573500 & -1.37412100 \\
\hline $\mathrm{C}$ & -2.91551400 & 1.77173400 & -0.09541500 \\
\hline $\mathrm{H}$ & -3.57384900 & 1.99062700 & 0.75450100 \\
\hline $\mathrm{H}$ & -3.37753300 & 2.24138500 & -0.96942700 \\
\hline $\mathrm{C}$ & -1.55446400 & -0.12266000 & 1.91698900 \\
\hline $\mathrm{H}$ & -2.07743200 & 0.76310300 & 2.29079400 \\
\hline
\end{tabular}




$\begin{array}{lrrr}\mathrm{H} & -2.12241400 & -1.00228300 & 2.23587200 \\ \mathrm{H} & -0.58617000 & -0.16529800 & 2.42410500 \\ \mathrm{C} & -4.02862700 & -0.57192400 & 0.15437600 \\ \mathrm{C} & -5.28915000 & -0.07721800 & -0.59296300 \\ \mathrm{H} & -5.36414300 & 1.01248400 & -0.48806900 \\ \mathrm{H} & -5.16314100 & -0.26999300 & -1.66992200 \\ \mathrm{C} & -6.61667500 & -0.69115700 & -0.12130400 \\ \mathrm{H} & -6.69391100 & -0.57723200 & 0.96900000 \\ \mathrm{H} & -6.62837400 & -1.77140300 & -0.31917800 \\ \mathrm{C} & -7.83447900 & -0.05052000 & -0.80369800 \\ \mathrm{H} & -7.87214000 & 1.02096700 & -0.55238700 \\ \mathrm{H} & -7.69003700 & -0.09908500 & -1.89322400 \\ \mathrm{C} & -9.19501300 & -0.69137100 & -0.46391200 \\ \mathrm{O} & 1.22280000 & 3.31866200 & -0.56162300 \\ \mathrm{C} & -3.84291000 & -2.08838000 & -0.02270100 \\ \mathrm{H} & -3.74253400 & -2.34661300 & -1.08569900 \\ \mathrm{H} & -2.94547300 & -2.45164800 & 0.48741900 \\ \mathrm{H} & -4.69842500 & -2.64582300 & 0.37277600 \\ \mathrm{C} & -9.55507100 & -0.55619600 & 1.02366300 \\ \mathrm{H} & -8.81867800 & -1.04249600 & 1.67234300 \\ \mathrm{H} & -9.61197700 & 0.50093900 & 1.31615100 \\ \mathrm{H} & -10.53085700 & -1.01043000 & 1.23361600 \\ \mathrm{C} & -10.30546000 & -0.09453000 & -1.34173400 \\ \mathrm{H} & -10.08455500 & -0.22208600 & -2.40830800 \\ \mathrm{H} & -11.27259600 & -0.57031800 & -1.13960600 \\ \mathrm{H} & -10.41923000 & 0.98103500 & -1.15220300 \\ \mathrm{C} & -1.48479100 & 2.32964800 & 0.15353600 \\ \mathrm{H} & -1.23708900 & 3.17521800 & -0.48786900 \\ \mathrm{H} & -1.37027600 & 2.67714600 & 1.18763900 \\ \mathrm{H} & -9.12404400 & -1.76547300 & -0.69737000 \\ \mathrm{H} & -4.18800100 & -0.37079800 & 1.22510400 \\ \mathrm{H} & -0.97271800 & -1.76418800 & -1.06303100 \\ & & & \\ & & & \\ \mathrm{H} & & & \\ \mathrm{H} & & & \end{array}$

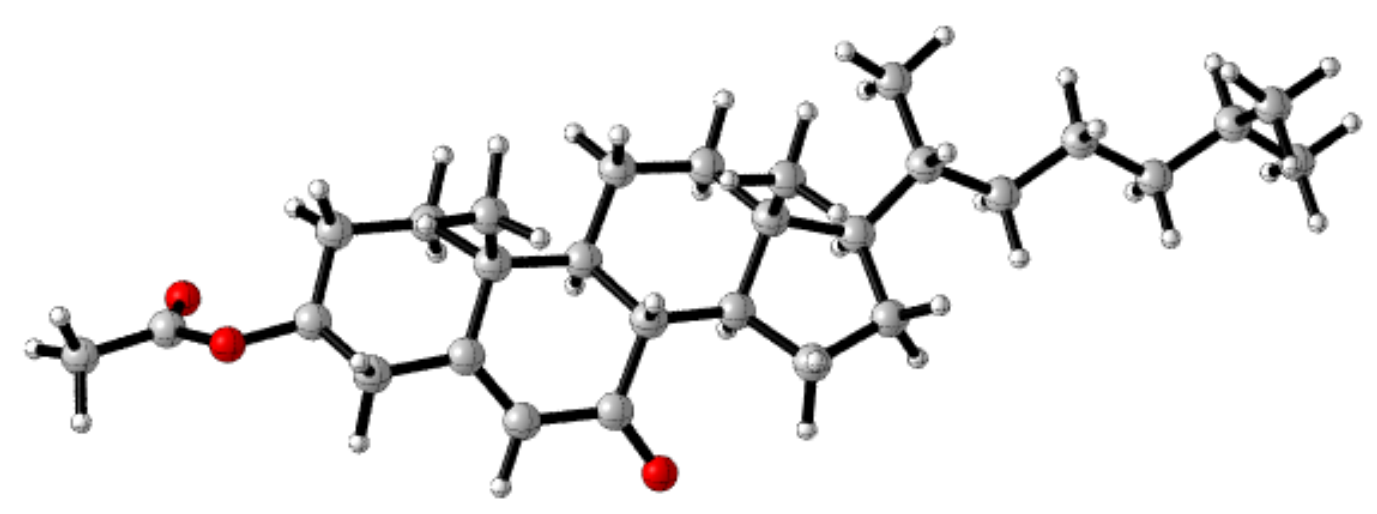

Figure S79. Optimized Structure of Steroid Radical at $\mathrm{H}_{\mathrm{c}}$ Site. 
Zero-point correction $=$

Thermal correction to Energy=

Thermal correction to Enthalpy=

Thermal correction to Gibbs Free Energy=

Sum of electronic and zero-point Energies=

Sum of electronic and thermal Energies=

Sum of electronic and thermal Enthalpies=

Sum of electronic and thermal Free Energies=
0.692793 (Hartree/Particle)

0.726725

0.727669

0.624725

$-1357.142749$

$-1357.108817$

$-1357.107873$

$-1357.210817$

Number of Imaginary Frequencies $=0$

E (Single Point Energy) [IEFPCM $\left(\mathrm{ICH}_{3 \mathrm{CN}} \mathrm{U} \omega \mathrm{BB} 97 \mathrm{X}-\mathrm{D} / 6-311++\mathrm{G}(2 \mathrm{~d}, 2 \mathrm{p})\right]=-1357.87748951$

02

$\begin{array}{lcrr}\text { C } & 6.02164100 & -0.13297300 & -0.12978500 \\ \mathrm{C} & 5.33331400 & 1.08460400 & 0.40131200 \\ \mathrm{C} & 3.82414300 & 1.04027500 & 0.25515500 \\ \mathrm{C} & 3.12005500 & -0.28639000 & 0.53752900 \\ \mathrm{C} & 3.88635900 & -1.40900400 & -0.22499100 \\ \mathrm{C} & 5.39642200 & -1.47664000 & 0.05429700 \\ \mathrm{H} & 3.44294700 & -2.37969600 & 0.01906700 \\ \mathrm{H} & 3.74169900 & -1.25479700 & -1.30198400 \\ \mathrm{H} & 5.57858700 & -1.83604000 & 1.08480100 \\ \mathrm{H} & 5.86091400 & -2.20789300 & -0.61270100 \\ \mathrm{C} & 1.64662800 & -0.23581900 & 0.00219500 \\ \mathrm{H} & 1.73351200 & -0.20293900 & -1.09600800 \\ \mathrm{C} & 3.15862900 & 2.15894400 & -0.08485800 \\ \mathrm{H} & 3.69261700 & 3.07576600 & -0.32674700 \\ \mathrm{C} & 1.69058800 & 2.26857700 & -0.14111800 \\ \mathrm{C} & 0.89947100 & 1.07341400 & 0.39642500 \\ \mathrm{H} & 0.90896300 & 1.18074900 & 1.49303900 \\ \mathrm{O} & 7.40831800 & 0.00038300 & -0.05540200 \\ \mathrm{C} & 8.22774800 & -0.71784900 & -0.88661400 \\ \mathrm{O} & 7.83314800 & -1.45967900 & -1.75374700 \\ \mathrm{C} & 9.67226400 & -0.43712000 & -0.55020400 \\ \mathrm{H} & 10.31499800 & -0.93721400 & -1.27530500 \\ \mathrm{H} & 9.86326300 & 0.64054100 & -0.55704700 \\ \mathrm{H} & 9.90068000 & -0.80283800 & 0.45699400 \\ \mathrm{C} & 3.17694000 & -0.55440100 & 2.06460100 \\ \mathrm{H} & 2.56181700 & 0.16120100 & 2.62001900 \\ \mathrm{H} & 2.82485000 & -1.56350400 & 2.30121300 \\ \mathrm{H} & 4.19774000 & -0.46964600 & 2.44950100 \\ \mathrm{C} & 0.82461200 & -1.49879600 & 0.33948200 \\ \mathrm{H} & 1.30743500 & -2.37820100 & -0.10198700 \\ \mathrm{H} & 0.82161400 & -1.66739400 & 1.42221200 \\ & & & \end{array}$




$\begin{array}{lrrr}\mathrm{C} & -0.55612200 & 1.04617800 & -0.09768100 \\ \mathrm{H} & -0.50163600 & 0.96768700 & -1.19598700 \\ \mathrm{C} & -0.63232700 & -1.44286300 & -0.15755600 \\ \mathrm{H} & -0.64243100 & -1.41923900 & -1.25723200 \\ \mathrm{H} & -1.13194800 & -2.37133500 & 0.13695200 \\ \mathrm{C} & -1.36870600 & -0.19850800 & 0.37304500 \\ \mathrm{C} & -2.75796200 & 0.12191700 & -0.27985800 \\ \mathrm{H} & -2.63891700 & -0.06796200 & -1.35873000 \\ \mathrm{C} & -2.90211500 & 1.66769800 & -0.07394400 \\ \mathrm{H} & -3.58545600 & 1.88073400 & 0.75754500 \\ \mathrm{H} & -3.34082800 & 2.13870100 & -0.95924200 \\ \mathrm{C} & -1.50944700 & -0.26275300 & 1.91139200 \\ \mathrm{H} & -2.09068400 & 0.57707200 & 2.30451600 \\ \mathrm{H} & -2.02206300 & -1.18263600 & 2.21429200 \\ \mathrm{H} & -0.54297300 & -0.25139100 & 2.42423400 \\ \mathrm{C} & -4.02053000 & -0.65673100 & 0.17487400 \\ \mathrm{H} & -4.19647700 & -0.42063600 & 1.23590200 \\ \mathrm{C} & -5.25996900 & -0.16358600 & -0.61264100 \\ \mathrm{H} & -5.27749300 & 0.93274100 & -0.60873800 \\ \mathrm{H} & -5.15149000 & -0.46019900 & -1.66764100 \\ \mathrm{C} & -6.61718300 & -0.65393600 & -0.08327700 \\ \mathrm{H} & -6.68589200 & -0.41794300 & 0.98768500 \\ \mathrm{H} & -6.68988900 & -1.74697700 & -0.16220200 \\ \mathrm{C} & -7.80073000 & -0.02731000 & -0.83565100 \\ \mathrm{H} & -7.77776000 & 1.06604900 & -0.70669100 \\ \mathrm{H} & -7.66431900 & -0.20625100 & -1.91249000 \\ \mathrm{H} & -1.38348200 & 2.53716800 & 1.26007000 \\ \mathrm{C} & -9.19312700 & -0.54905700 & -0.42774300 \\ \mathrm{H} & -9.18269600 & -1.64506200 & -0.53703100 \\ \mathrm{O} & 1.16690900 & 3.28876600 & -0.57105000 \\ \mathrm{C} & -3.87873300 & -2.18307800 & 0.04705000 \\ \mathrm{H} & -3.60487300 & -2.46874200 & -0.97694900 \\ \mathrm{H} & -3.11714200 & -2.58500400 & 0.72041900 \\ \mathrm{H} & -4.81756400 & -2.69070000 & 0.29095200 \\ \mathrm{C} & -9.53896700 & -0.22532400 & 1.03389800 \\ \mathrm{H} & -8.82846400 & -0.67510500 & 1.73564000 \\ \mathrm{H} & -9.53528700 & 0.85975500 & 1.20380200 \\ \mathrm{H} & -10.53783000 & -0.59716500 & 1.29188200 \\ \mathrm{H} & -10.27255300 & 0.00441300 & -1.37030000 \\ \mathrm{H} & -10.06383500 & -0.25645100 & -2.41482000 \\ \mathrm{H} & -1.26372100 & -0.39048200 & -1.11688800 \\ \mathrm{H} & & 1.98721300 & -0.07836500 \\ \mathrm{H} & -1.240796900 & 1.09924500 & -1.30537400 \\ \mathrm{H} & -1.19769800 & 1.47770100\end{array}$




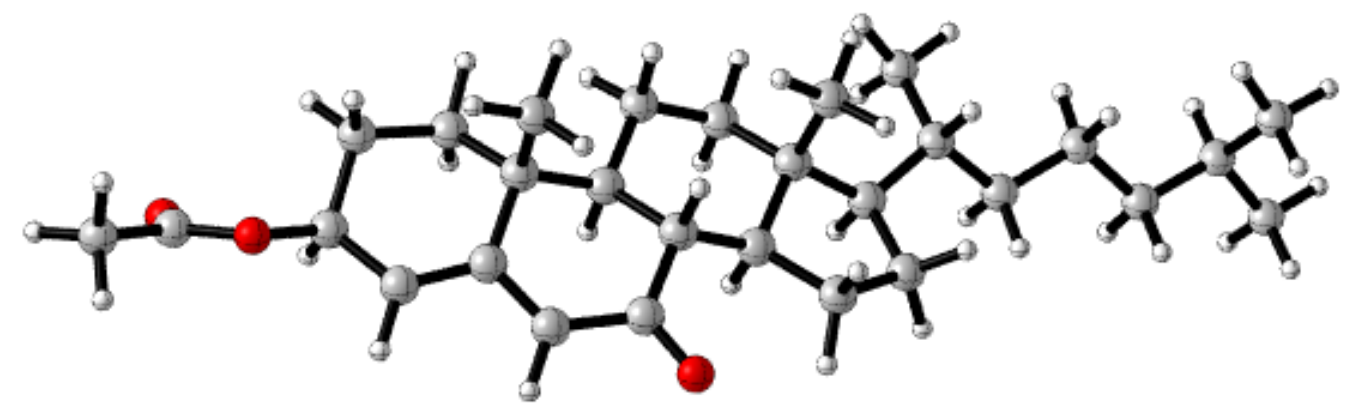

Figure S80. Optimized Structure of Steroid Radical at $\mathrm{H}_{\mathrm{d}}$ Site.

Zero-point correction $=$

Thermal correction to Energy=

Thermal correction to Enthalpy=

Thermal correction to Gibbs Free Energy=

Sum of electronic and zero-point Energies $=$

Sum of electronic and thermal Energies=

Sum of electronic and thermal Enthalpies $=$

Sum of electronic and thermal Free Energies=
0.693518 (Hartree/Particle)

0.727129

0.728074

0.626039

$-1357.166908$

$-1357.133297$

$-1357.132352$

$-1357.234387$

Number of Imaginary Frequencies $=0$

E (Single Point Energy) $\left[\operatorname{IEFPCM}_{(\mathrm{CH} 3 \mathrm{CN})} \mathrm{U \omega B}\right.$ B97X-D/6-311++G(2d,2p)] = -1357.90074556

02

$\begin{array}{lrrr}\mathrm{C} & 5.91572400 & -0.37143800 & -0.64203400 \\ \mathrm{C} & 5.11129300 & 0.88548800 & -0.59018100 \\ \mathrm{C} & 3.81656300 & 0.96807500 & -0.11332600 \\ \mathrm{C} & 3.13509200 & -0.21341000 & 0.59038400 \\ \mathrm{C} & 3.83676900 & -1.53264900 & 0.17485100 \\ \mathrm{C} & 5.36766400 & -1.46541100 & 0.27229000 \\ \mathrm{H} & 5.95060400 & -0.74321300 & -1.67551400 \\ \mathrm{H} & 3.47715600 & -2.35586000 & 0.80114100 \\ \mathrm{H} & 3.55267800 & -1.78022000 & -0.85826300 \\ \mathrm{H} & 5.68065000 & -1.24794100 & 1.29951000 \\ \mathrm{H} & 5.81384900 & -2.42580300 & -0.00460400 \\ \mathrm{C} & 1.63491100 & -0.22243100 & 0.14339600 \\ \mathrm{H} & 1.65732900 & -0.27056000 & -0.95810900 \\ \mathrm{C} & 3.09310900 & 2.16052100 & -0.28627200 \\ \mathrm{H} & 3.58387500 & 3.03583600 & -0.70608100 \\ \mathrm{C} & 1.68540800 & 2.32834400 & 0.02598300 \\ \mathrm{C} & 0.89642900 & 1.09980800 & 0.50101700 \\ \mathrm{H} & 0.84031400 & 1.19815600 & 1.59532100 \\ \mathrm{O} & 7.28351300 & -0.02739600 & -0.27778800 \\ \mathrm{C} & 8.26788800 & -0.76489900 & -0.85099300 \\ \mathrm{O} & 8.06883100 & -1.67186900 & -1.62914900\end{array}$




$\begin{array}{lccc}\mathrm{C} & 9.62495200 & -0.29494000 & -0.38219200 \\ \mathrm{H} & 10.40203000 & -0.87990700 & -0.87505900 \\ \mathrm{H} & 9.75547200 & 0.76824700 & -0.60803100 \\ \mathrm{H} & 9.70598600 & -0.40959400 & 0.70382900 \\ \mathrm{C} & 3.27465800 & -0.01377200 & 2.12190000 \\ \mathrm{H} & 2.84912300 & 0.93891800 & 2.44965700 \\ \mathrm{H} & 2.77258500 & -0.81874700 & 2.66980400 \\ \mathrm{H} & 4.32617200 & -0.01027600 & 2.42387200 \\ \mathrm{C} & 0.83667900 & -1.45306900 & 0.61776200 \\ \mathrm{H} & 1.31856600 & -2.36859300 & 0.25663200 \\ \mathrm{H} & 0.85298800 & -1.51355400 & 1.71336500 \\ \mathrm{C} & -0.54280200 & 1.04311000 & -0.05091300 \\ \mathrm{H} & -0.44731900 & 0.85749100 & -1.13404500 \\ \mathrm{C} & -0.62404700 & -1.45122400 & 0.12434300 \\ \mathrm{H} & -0.63033400 & -1.55217800 & -0.97108200 \\ \mathrm{H} & -1.12405300 & -2.34146700 & 0.51914600 \\ \mathrm{C} & -1.37164000 & -0.15859500 & 0.50651900 \\ \mathrm{C} & -2.73678200 & 0.09282900 & -0.22415900 \\ \mathrm{H} & -2.57819600 & -0.20106400 & -1.27434700 \\ \mathrm{C} & -2.88894900 & 1.65004900 & -0.17562900 \\ \mathrm{H} & -3.60212100 & 1.94026200 & 0.60591900 \\ \mathrm{H} & -3.29609300 & 2.03056700 & -1.11780200 \\ \mathrm{C} & -1.56657800 & -0.07987000 & 2.03752400 \\ \mathrm{H} & -2.12620600 & 0.81229000 & 2.33531100 \\ \mathrm{H} & -2.12499300 & -0.95038900 & 2.39893500 \\ \mathrm{H} & -0.61799500 & -0.06142700 & 2.58264200 \\ \mathrm{H} & -4.01572400 & -0.64251100 & 0.25668000 \\ \mathrm{C} & -4.23067900 & -0.30586300 & 1.28277300 \\ \mathrm{H} & -3.81823200 & -2.65792200 & 0.53794600 \\ \mathrm{C} & -5.22469700 & -0.23096600 & -0.62010300 \\ \mathrm{H} & -5.24208800 & 0.86059500 & -0.72186400 \\ \mathrm{H} & -5.07681100 & -0.62695400 & -1.63704800 \\ \mathrm{C} & -6.60116200 & -0.67265900 & -0.09765800 \\ \mathrm{H} & -6.71144800 & -0.33307900 & 0.94151400 \\ \mathrm{H} & -6.67057700 & -1.76846600 & -0.07179300 \\ \mathrm{C} & -7.75457100 & -0.12733900 & -0.95313500 \\ \mathrm{H} & -7.73651100 & 0.97347300 & -0.93141600 \\ \mathrm{H} & -7.57639700 & -0.41062500 & -2.00110800 \\ \mathrm{H} & -9.16185900 & -0.61165000 & -0.55024900 \\ \mathrm{H} & -9.14734100 & -1.71304300 & -0.55077800 \\ \mathrm{H} & -3.16335500 & 3.44245200 & -0.08473900 \\ \mathrm{H} & -0.14708800 & 0.85789100 \\ \mathrm{H} & -2.1344900 & -2.17368600 & 0.28092300 \\ \mathrm{H} & -2.52303400 & 1.62759000\end{array}$




$\begin{array}{lccc}\mathrm{H} & -9.56742500 & 0.94943600 & 0.92026100 \\ \mathrm{H} & -10.57269100 & -0.49555400 & 1.11213800 \\ \mathrm{C} & -10.20357800 & -0.15742100 & -1.58389900 \\ \mathrm{H} & -9.95413900 & -0.51859000 & -2.58888400 \\ \mathrm{H} & -11.20388100 & -0.52941200 & -1.33175900 \\ \mathrm{H} & -10.25906300 & 0.93829300 & -1.62873600 \\ \mathrm{C} & -1.48454800 & 2.24704900 & 0.10655600 \\ \mathrm{H} & -1.22491500 & 3.06200000 & -0.56917200 \\ \mathrm{H} & -1.42132900 & 2.65968600 & 1.11971100 \\ \mathrm{H} & 5.56188500 & 1.76416800 & -1.04809800\end{array}$

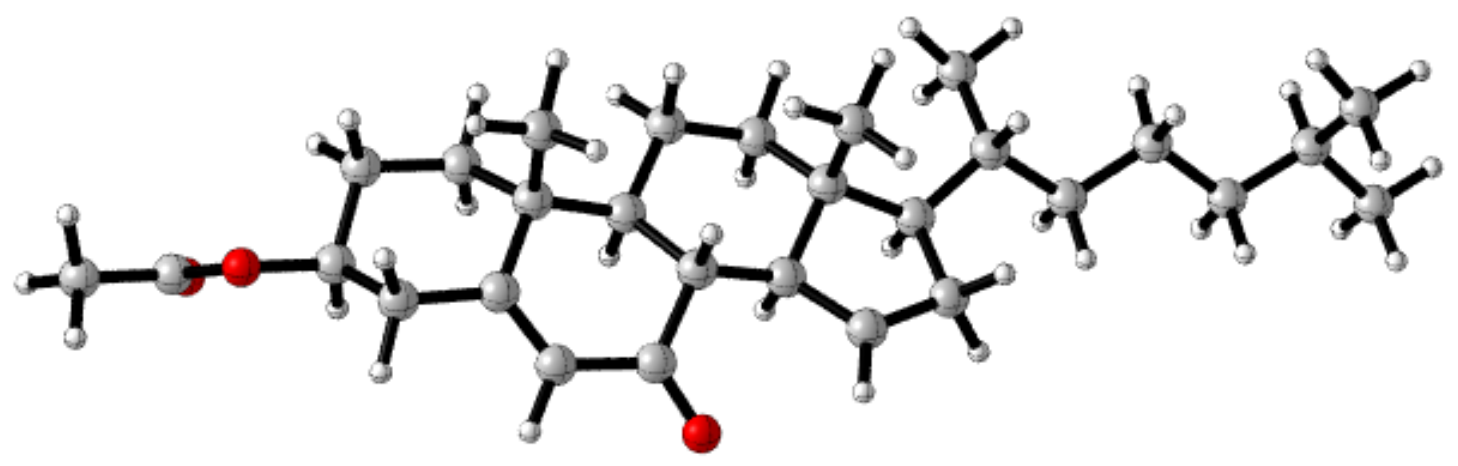

Figure S81. Optimized Structure of Steroid Radical at $\mathrm{H}_{\mathrm{e}}$ Site.

Zero-point correction=

Thermal correction to Energy=

Thermal correction to Enthalpy=

Thermal correction to Gibbs Free Energy=

Sum of electronic and zero-point Energies=

Sum of electronic and thermal Energies $=$

Sum of electronic and thermal Enthalpies=

Sum of electronic and thermal Free Energies=
0.692098 (Hartree/Particle)

0.725957

0.726901

0.624456

$-1357.143968$

$-1357.110109$

$-1357.109165$

$-1357.211611$

Number of Imaginary Frequencies $=0$

E (Single Point Energy) [IEFPCM $(\mathrm{CH} 3 \mathrm{CN}) \mathrm{U} \omega \mathrm{B} 97 \mathrm{X}-\mathrm{D} / 6-311++\mathrm{G}(2 \mathrm{~d}, 2 \mathrm{p})]=-1357.87909209$

02

$\begin{array}{lrrr}\mathrm{C} & 5.95517100 & -0.13705000 & -0.33876900 \\ \mathrm{C} & 5.31231100 & 1.02657100 & 0.41696400 \\ \mathrm{C} & 3.80132200 & 1.01104500 & 0.31839600 \\ \mathrm{C} & 3.08130400 & -0.30450300 & 0.62492800 \\ \mathrm{C} & 3.84084300 & -1.46697400 & -0.08664600 \\ \mathrm{C} & 5.36344000 & -1.46771700 & 0.11227000 \\ \mathrm{H} & 5.61447100 & 0.94421300 & 1.47150400\end{array}$




\begin{tabular}{|c|c|c|c|}
\hline $\mathrm{H}$ & 5.70475800 & 1.98089600 & 0.05140800 \\
\hline $\mathrm{H}$ & 5.82129100 & -0.01946100 & -1.41866400 \\
\hline $\mathrm{H}$ & 3.43737200 & -2.42532400 & 0.25665600 \\
\hline $\mathrm{H}$ & 3.63138500 & -1.40999000 & -1.16400900 \\
\hline $\mathrm{H}$ & 5.62464600 & -1.63378900 & 1.16532900 \\
\hline $\mathrm{H}$ & 5.81080800 & -2.28266400 & -0.46602400 \\
\hline $\mathrm{C}$ & 1.61733400 & -0.24565600 & 0.06793600 \\
\hline $\mathrm{H}$ & 1.72390200 & -0.19914000 & -1.02814900 \\
\hline $\mathrm{C}$ & 3.14020000 & 2.13880500 & -0.00319900 \\
\hline $\mathrm{H}$ & 3.68006300 & 3.05248900 & -0.24373600 \\
\hline $\mathrm{C}$ & 1.67270600 & 2.26850300 & -0.02967500 \\
\hline $\mathrm{C}$ & 0.86918600 & 1.06196200 & 0.46152400 \\
\hline $\mathrm{H}$ & 0.83531900 & 1.15531100 & 1.55773900 \\
\hline $\mathrm{O}$ & 7.37546100 & -0.07448300 & -0.05401000 \\
\hline $\mathrm{C}$ & 8.21251200 & -0.62311300 & -0.96963000 \\
\hline $\mathrm{O}$ & 7.84206700 & -1.16209500 & -1.98933300 \\
\hline $\mathrm{C}$ & 9.65149800 & -0.46136100 & -0.53769900 \\
\hline $\mathrm{H}$ & 10.30722500 & -0.90952400 & -1.28480800 \\
\hline $\mathrm{H}$ & 9.89224500 & 0.60013200 & -0.41864700 \\
\hline $\mathrm{H}$ & 9.80939300 & -0.94212200 & 0.43334800 \\
\hline $\mathrm{C}$ & 3.11204200 & -0.52611800 & 2.16074000 \\
\hline $\mathrm{H}$ & 2.52915800 & 0.23579800 & 2.68810700 \\
\hline $\mathrm{H}$ & 2.70842100 & -1.50887200 & 2.42436700 \\
\hline $\mathrm{H}$ & 4.13260800 & -0.48039000 & 2.55218300 \\
\hline $\mathrm{C}$ & 0.78848600 & -1.51379500 & 0.37353300 \\
\hline $\mathrm{H}$ & 1.27818500 & -2.38424800 & -0.07789200 \\
\hline $\mathrm{H}$ & 0.77552500 & -1.70175300 & 1.45292900 \\
\hline $\mathrm{C}$ & -0.56649500 & 1.03093300 & -0.09145300 \\
\hline $\mathrm{H}$ & -0.44497300 & 0.90346000 & -1.18947800 \\
\hline $\mathrm{C}$ & -0.66617300 & -1.45876300 & -0.13704400 \\
\hline $\mathrm{H}$ & -0.66783900 & -1.44733400 & -1.23674400 \\
\hline $\mathrm{H}$ & -1.16842800 & -2.38362100 & 0.16414800 \\
\hline $\mathrm{C}$ & -1.40216200 & -0.20727900 & 0.37575100 \\
\hline $\mathrm{C}$ & -2.78758800 & 0.12827800 & -0.28709900 \\
\hline $\mathrm{H}$ & -2.66130600 & -0.04758200 & -1.36776000 \\
\hline $\mathrm{C}$ & -2.91465400 & 1.67306900 & -0.06965200 \\
\hline $\mathrm{H}$ & -3.55053400 & 1.89196700 & 0.80601900 \\
\hline $\mathrm{H}$ & -3.41573000 & 2.16355900 & -0.91792400 \\
\hline $\mathrm{C}$ & -1.55405200 & -0.24765900 & 1.91245800 \\
\hline $\mathrm{H}$ & -2.05331800 & 0.65065900 & 2.28899100 \\
\hline $\mathrm{H}$ & -2.14710600 & -1.11563700 & 2.22153500 \\
\hline $\mathrm{H}$ & -0.59235900 & -0.31799500 & 2.42867700 \\
\hline $\mathrm{C}$ & -4.05738300 & -0.64404300 & 0.15548800 \\
\hline $\mathrm{H}$ & -4.23426600 & -0.41730700 & 1.2182100 \\
\hline $\mathrm{C}$ & -5.29148500 & -0.13315800 & -0.62862200 \\
\hline $\mathrm{H}$ & -5.30718100 & 0.96295000 & -0.6024650 \\
\hline
\end{tabular}




$\begin{array}{lccc}\mathrm{H} & -5.17888500 & -0.40850300 & -1.68890100 \\ \mathrm{C} & -6.65220600 & -0.63092200 & -0.11532500 \\ \mathrm{H} & -6.72264000 & -0.42251900 & 0.96121800 \\ \mathrm{H} & -6.72949500 & -1.72114500 & -0.22300200 \\ \mathrm{C} & -7.83089000 & 0.02052100 & -0.85413700 \\ \mathrm{H} & -7.80367900 & 1.10994300 & -0.69604000 \\ \mathrm{H} & -7.69219900 & -0.13034400 & -1.93497400 \\ \mathrm{C} & -9.22659200 & -0.50588600 & -0.46390300 \\ \mathrm{H} & -9.22064600 & -1.59857500 & -0.60269600 \\ \mathrm{O} & 1.15573700 & 3.31606300 & -0.39750000 \\ \mathrm{C} & -3.92378200 & -2.16966600 & 0.01307300 \\ \mathrm{H} & -3.64823200 & -2.44701500 & -1.01278800 \\ \mathrm{H} & -3.16606900 & -2.58132000 & 0.68490700 \\ \mathrm{H} & -4.86582000 & -2.67458200 & 0.24965700 \\ \mathrm{C} & -9.57485100 & -0.22010200 & 1.00506000 \\ \mathrm{H} & -8.86827200 & -0.69180100 & 1.69630200 \\ \mathrm{H} & -9.56692500 & 0.85996600 & 1.20419400 \\ \mathrm{H} & -10.57600700 & -0.59432400 & 1.25033900 \\ \mathrm{C} & -10.30104600 & 0.07761400 & -1.39394200 \\ \mathrm{H} & -10.09067000 & -0.15581800 & -2.44459900 \\ \mathrm{H} & -11.29455100 & -0.31966500 & -1.15382500 \\ \mathrm{H} & -10.34947000 & 1.17050300 & -1.29952400 \\ \mathrm{C} & -1.51267500 & 2.16733800 & 0.11819100 \\ \mathrm{H} & -1.22421900 & 3.20938300 & 0.11452700\end{array}$

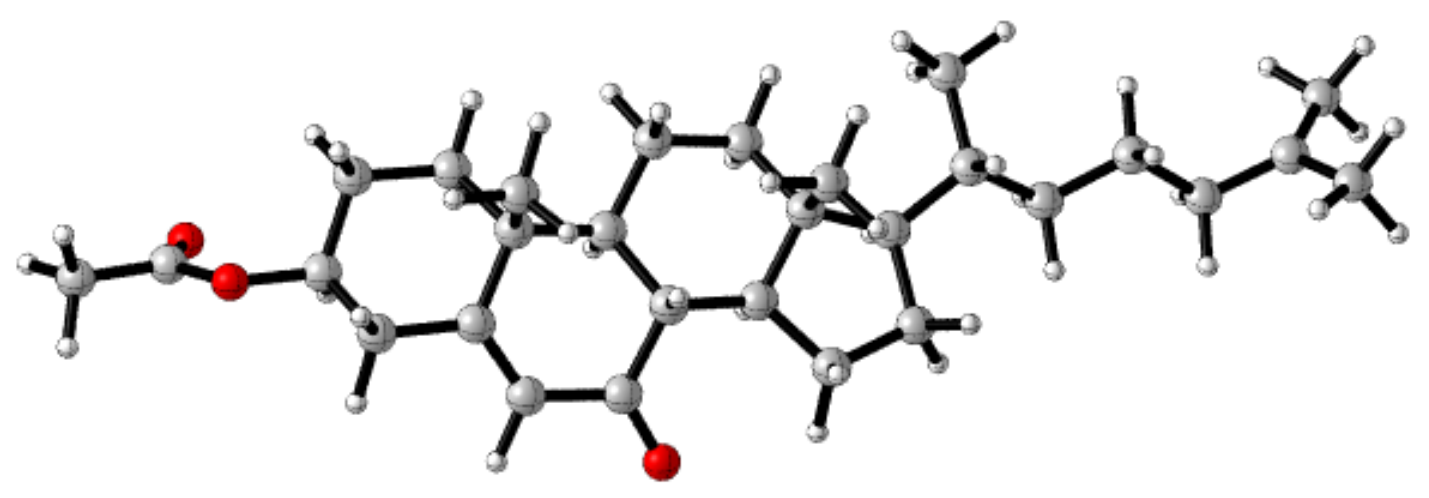

Figure S82. Optimized Structure of Steroid Radical at $\mathrm{H}_{\mathrm{f}}$ Site.

Zero-point correction $=$

Thermal correction to Energy=

Thermal correction to Enthalpy=

Thermal correction to Gibbs Free Energy=

Sum of electronic and zero-point Energies $=$

Sum of electronic and thermal Energies $=$

Sum of electronic and thermal Enthalpies $=$

Sum of electronic and thermal Free Energies=
0.691994 (Hartree/Particle)

0.726550

0.727494

0.621246

$-1357.147715$

$-1357.113158$

$-1357.112214$

$-1357.218462$ 
Number of Imaginary Frequencies $=0$

E (Single Point Energy) $\left[\operatorname{IEFPCM}_{(\mathrm{CH} 3 \mathrm{CN})} \mathrm{U \omega B}\right.$ B7X-D/6-311++G(2d,2p)] = -1357.88175128

02

$\begin{array}{llll}\mathrm{C} & 5.92013200 & -0.20971900 & -0.31572700\end{array}$

$\begin{array}{llll}\mathrm{C} & 5.28670400 & 1.01887300 & 0.33868500\end{array}$

$\begin{array}{llll}\mathrm{C} & 3.77636600 & 1.01141800 & 0.23025600\end{array}$

$\begin{array}{llll}\mathrm{C} & 3.04013700 & -0.26541000 & 0.64258600\end{array}$

C $\quad 3.78802200 \quad-1.49030900 \quad 0.03017300$

C $\quad 5.30967200 \quad-1.49304500 \quad 0.23661800$

$\mathrm{H} \quad \begin{array}{llll}\mathrm{H} & 5.58161700 & 1.01932300 & 1.39841800\end{array}$

$\mathrm{H} \quad 5.69247800 \quad 1.93563700 \quad-0.10094400$

$\mathrm{H} \quad 5.79442400 \quad-0.17800300 \quad-1.40250100$

$\mathrm{H} \quad 3.37140800 \quad-2.41328300 \quad 0.44694200$

$\mathrm{H} \quad 3.58369500 \quad-1.51711600 \quad-1.04931900$

$\mathrm{H} \quad 5.56407200 \quad-1.57781400 \quad 1.30097500$

$\mathrm{H} \quad 5.74980200 \quad-2.35665300 \quad-0.27247400$

C $\quad 1.57680100 \quad-0.23774100 \quad 0.08021100$

$\mathrm{H} \quad 1.68298200 \quad-0.29233100 \quad-1.01554800$

C $\quad 3.12978300 \quad 2.11176700 \quad-0.19728700$

$\mathrm{H} \quad 3.68086500 \quad 2.99388700 \quad-0.51715000$

$\begin{array}{llll}\mathrm{C} & 1.66340500 & 2.24794500 & -0.25885700\end{array}$

$\begin{array}{llll}\mathrm{C} & 0.84517500 & 1.10963400 & 0.35650300\end{array}$

$\mathrm{H} \quad \begin{array}{llll}\mathrm{H} & 0.83707700 & 1.30347200 & 1.44106700\end{array}$

O $\quad 7.33931500 \quad-0.14100200 \quad-0.02664200$

C $\quad 8.17552200 \quad-0.77084300 \quad-0.88918500$

O $\quad 7.80528300 \quad-1.38541500 \quad-1.86527700$

C $\quad 9.61352800 \quad-0.59188000-0.46070500$

$\mathrm{H} \quad 10.26872900-1.10462300 \quad-1.16555000$

$\mathrm{H} \quad 9.86603500 \quad 0.47288300 \quad-0.42324500$

$\mathrm{H} \quad 9.75942700 \quad-0.99689600 \quad 0.54611900$

$\begin{array}{llll}\mathrm{C} & 3.06678000 & -0.36138300 & 2.19135600\end{array}$

$\mathrm{H} \quad 2.48786200 \quad 0.44444100 \quad 2.65392100$

$\mathrm{H} \quad 2.65655200 \quad-1.31685500 \quad 2.53326100$

$\mathrm{H} \quad 4.08681000 \quad-0.28998900 \quad 2.58016500$

C $\quad 0.73151500 \quad-1.46001300 \quad 0.49862700$

$\mathrm{H} \quad 1.20823100 \quad-2.37564500 \quad 0.13002500$

$\mathrm{H} \quad 0.71135700 \quad-1.54838400 \quad 1.59075700$

$\begin{array}{lllll}\mathrm{C} & -0.60296400 & 1.06429000 & -0.15871800\end{array}$

$\mathrm{H} \quad-0.53301100 \quad 0.89769400 \quad-1.24630700$

C $\quad-0.71726200 \quad-1.42089100 \quad-0.02345000$

$\mathrm{H} \quad-0.71122500 \quad-1.48320300 \quad-1.12166500$

$\mathrm{H} \quad-1.23637300 \quad-2.31570100 \quad 0.33458700$

C $\quad-1.44107400 \quad-0.12784200 \quad 0.39634700$

C $\quad-2.81608300 \quad 0.15929400 \quad-0.30083900$

$\mathrm{H} \quad-2.68530500 \quad-0.11792900-1.35926800$ 


$\begin{array}{lccc}\mathrm{C} & -2.93950100 & 1.71840100 & -0.22089400 \\ \mathrm{H} & -3.63130700 & 2.00675700 & 0.58033400 \\ \mathrm{H} & -3.35819200 & 2.12344600 & -1.14755700 \\ \mathrm{C} & -1.60373500 & -0.06805200 & 1.93262100 \\ \mathrm{H} & -2.17664200 & 0.80890700 & 2.24949400 \\ \mathrm{H} & -2.13505400 & -0.95329700 & 2.29970700 \\ \mathrm{H} & -0.64412900 & -0.03060600 & 2.45696700 \\ \mathrm{C} & -4.09649000 & -0.56273900 & 0.19582100 \\ \mathrm{H} & -4.28352500 & -0.23970800 & 1.23169200 \\ \mathrm{C} & -5.31754600 & -0.11775600 & -0.64746500 \\ \mathrm{H} & -5.31496300 & 0.97484200 & -0.74044700 \\ \mathrm{H} & -5.20344300 & -0.50835900 & -1.67088800 \\ \mathrm{C} & -6.68814100 & -0.53552700 & -0.09350200 \\ \mathrm{H} & -6.78355500 & -0.18826000 & 0.94538900 \\ \mathrm{H} & -6.78315300 & -1.62777200 & -0.06176600 \\ \mathrm{C} & -7.86501900 & 0.03394300 & -0.92552700 \\ \mathrm{H} & -7.75663600 & 1.13013800 & -0.96255000 \\ \mathrm{H} & -7.75885800 & -0.31955100 & -1.96135000 \\ \mathrm{C} & -9.21927400 & -0.33616900 & -0.38827700 \\ \mathrm{O} & 1.16208300 & 3.24741700 & -0.75844500 \\ \mathrm{C} & -3.97614700 & -2.09626000 & 0.19242300 \\ \mathrm{H} & -3.69027600 & -2.46650100 & -0.80074700 \\ \mathrm{H} & -3.23178000 & -2.45383800 & 0.90872100 \\ \mathrm{H} & -4.92663700 & -2.56919100 & 0.45958300 \\ \mathrm{C} & -9.85155500 & 0.51111800 & 0.67279400 \\ \mathrm{H} & -9.10244200 & 1.04058300 & 1.27445700 \\ \mathrm{H} & -10.51698300 & 1.28548600 & 0.24653000 \\ \mathrm{H} & -10.47386900 & -0.08276900 & 1.35645900 \\ \mathrm{C} & -10.03910400 & -1.39345500 & -1.06033300 \\ \mathrm{H} & -9.41179900 & -2.13112700 & -1.57605500 \\ \mathrm{H} & -10.67723900 & -1.93263100 & -0.34656400 \\ \mathrm{H} & -10.72149500 & -0.97344300 & -1.82367300 \\ \mathrm{C} & -1.51792300 & 2.28305100 & 0.04029400 \\ \mathrm{H} & -1.25236700 & 3.10168900 & -0.62743300 \\ \mathrm{H} & -1.42696400 & 2.67010600 & 1.06304600\end{array}$




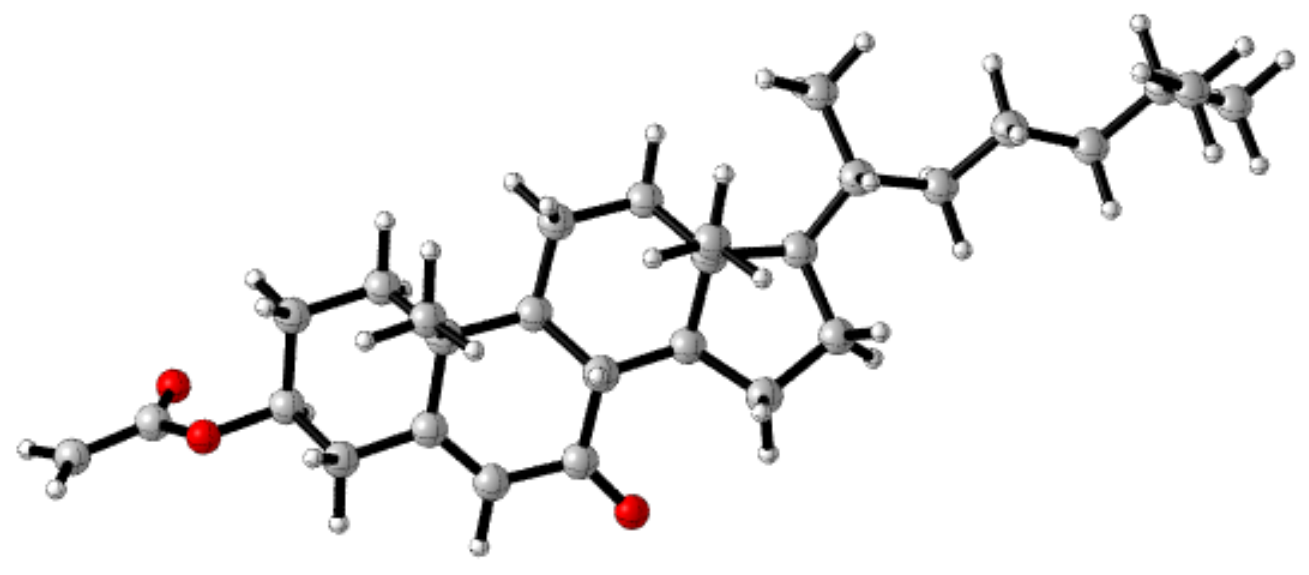

Figure S83. Optimized Structure of Steroid Radical at $\mathrm{H}_{\mathrm{h}}$ Site.

Zero-point correction $=$

Thermal correction to Energy=

Thermal correction to Enthalpy=

Thermal correction to Gibbs Free Energy=

Sum of electronic and zero-point Energies=

Sum of electronic and thermal Energies $=$

Sum of electronic and thermal Enthalpies $=$

Sum of electronic and thermal Free Energies=
0.693548 (Hartree/Particle)

0.726875

0.727819

0.627047

$-1357.142053$

$-1357.108727$

$-1357.107783$

$-1357.208555$

Number of Imaginary Frequencies $=0$

E (Single Point Energy) [IEFPCM $\left(\mathrm{ICH}_{3 \mathrm{CN})} \mathrm{U} \omega \mathrm{B} 97 \mathrm{X}-\mathrm{D} / 6-311++\mathrm{G}(2 \mathrm{~d}, 2 \mathrm{p})\right]=-1357.87864434$

02

$\mathrm{C}$

C

6.00320200

5.35896200

$-0.14680600$

$-0.34473600$

C

3.84826400

1.02448000

0.39850000

C

3.12887700

1.00679100

0.29750000

C

3.88759200

$-0.30469400$

0.62107600

C

5.41017300

$-1.47408200$

$-0.07995700$

$\mathrm{H}$

$3.48375100-2.42913600$

0.11908000

$\mathrm{H}$

$3.67803400-1.42676700$

0.27211400

$\mathrm{H}$

$5.67171600-1.62936400$

$-1.15774500$

$\mathrm{H}$

$5.85727500 \quad-2.29424100$

1.17358700

C

$1.66248900-0.25422500$

$-0.45123600$

$\mathrm{H}$

1.76378300

0.06831400

C

3.18665600

$-0.22883500$

$-1.02897900$

$\mathrm{H}$

3.72579900

2.12723900

$-0.04684100$

C

1.71852600

3.03642700

$-0.30535900$

C

0.91649800

2.25026200

$-0.09136700$

$\mathrm{H}$

0.91118000

1.06088500

0.44360700

$1.17619400 \quad 1.53930500$ 


\begin{tabular}{|c|c|c|c|}
\hline $\mathrm{O}$ & 7.42037400 & -0.08007800 & -0.06164600 \\
\hline $\mathrm{C}$ & 8.25526500 & -0.65446400 & -0.97319100 \\
\hline $\mathrm{O}$ & 7.87293400 & -1.22164300 & -1.98774300 \\
\hline $\mathrm{C}$ & 9.64408000 & -0.50746900 & -0.59303800 \\
\hline $\mathrm{H}$ & 10.40131200 & -0.92073100 & -1.24696400 \\
\hline $\mathrm{C}$ & 3.16447100 & -0.51009800 & 2.15902200 \\
\hline $\mathrm{H}$ & 2.57989700 & 0.25479500 & 2.68025500 \\
\hline $\mathrm{H}$ & 2.76565000 & -1.49165500 & 2.43403600 \\
\hline $\mathrm{H}$ & 4.18574600 & -0.45598200 & 2.54737200 \\
\hline $\mathrm{C}$ & 0.83356600 & -1.51327400 & 0.40148000 \\
\hline $\mathrm{H}$ & 1.31939300 & -2.39458400 & -0.03297400 \\
\hline $\mathrm{H}$ & 0.81906300 & -1.67925100 & 1.48460700 \\
\hline $\mathrm{C}$ & -0.53326700 & 1.03528400 & -0.06828900 \\
\hline $\mathrm{H}$ & -0.46603400 & 0.94806800 & -1.16528200 \\
\hline $\mathrm{C}$ & -0.61783700 & -1.45452700 & -0.11141100 \\
\hline $\mathrm{H}$ & -0.61576300 & -1.43817200 & -1.21128100 \\
\hline $\mathrm{H}$ & -1.12464500 & -2.37891400 & 0.18356100 \\
\hline $\mathrm{C}$ & -1.35515000 & -0.20362300 & 0.40235100 \\
\hline $\mathrm{C}$ & -2.73610500 & 0.11644300 & -0.26823300 \\
\hline $\mathrm{H}$ & -2.60583200 & -0.08216700 & -1.34424500 \\
\hline $\mathrm{C}$ & -2.87748000 & 1.66416500 & -0.07590900 \\
\hline $\mathrm{H}$ & -3.56945400 & 1.88587700 & 0.74610200 \\
\hline $\mathrm{H}$ & -3.30459200 & 2.12970800 & -0.96972400 \\
\hline $\mathrm{C}$ & -1.51261200 & -0.25643800 & 1.93943500 \\
\hline $\mathrm{H}$ & -2.09311000 & 0.58937800 & 2.32062600 \\
\hline $\mathrm{H}$ & -2.03374400 & -1.17127100 & 2.24302900 \\
\hline $\mathrm{H}$ & -0.55156300 & -0.24723900 & 2.46243000 \\
\hline $\mathrm{C}$ & -4.00627200 & -0.65449600 & 0.17847800 \\
\hline $\mathrm{H}$ & -4.19315900 & -0.40945800 & 1.23559500 \\
\hline $\mathrm{C}$ & -5.23520300 & -0.16349500 & -0.62669800 \\
\hline $\mathrm{H}$ & -5.24831600 & 0.93287300 & -0.63298400 \\
\hline $\mathrm{H}$ & -5.11668100 & -0.47018800 & -1.67773200 \\
\hline $\mathrm{C}$ & -6.59999100 & -0.64346900 & -0.10740900 \\
\hline $\mathrm{H}$ & -6.67967200 & -0.39601100 & 0.96019600 \\
\hline $\mathrm{H}$ & -6.67587000 & -1.73701600 & -0.17569500 \\
\hline $\mathrm{C}$ & -7.77276700 & -0.02045100 & -0.87940000 \\
\hline $\mathrm{H}$ & -7.74652400 & 1.07418700 & -0.76244500 \\
\hline $\mathrm{H}$ & -7.62562200 & -0.21203700 & -1.95264300 \\
\hline $\mathrm{C}$ & -9.17167000 & -0.53175000 & -0.48059100 \\
\hline $\mathrm{H}$ & -9.16473000 & -1.62897700 & -0.57714500 \\
\hline $\mathrm{O}$ & 1.20316400 & 3.27735400 & -0.51488900 \\
\hline $\mathrm{C}$ & -3.86845100 & -2.18227400 & 0.0641090 \\
\hline $\mathrm{H}$ & -3.58378800 & -2.47681500 & -0.95441200 \\
\hline $\mathrm{H}$ & -3.11624600 & -2.58174700 & 0.7493950 \\
\hline $\mathrm{H}$ & -4.81191400 & -2.68462100 & 0.3009240 \\
\hline $\mathrm{C}$ & -9.53183600 & -0.18969000 & 0.9733940 \\
\hline
\end{tabular}




$\begin{array}{lccc}\mathrm{H} & -8.83089400 & -0.63427100 & 1.68794600 \\ \mathrm{H} & -9.52534500 & 0.89725700 & 1.13079900 \\ \mathrm{H} & -10.53500200 & -0.55436500 & 1.22481000 \\ \mathrm{C} & -10.23851000 & 0.01528000 & -1.44107000 \\ \mathrm{H} & -10.01972400 & -0.25855300 & -2.48019700 \\ \mathrm{H} & -11.23404000 & -0.37247200 & -1.19381200 \\ \mathrm{H} & -10.28739400 & 1.11100300 & -1.38936000 \\ \mathrm{C} & -1.46161500 & 2.22553700 & 0.22082500 \\ \mathrm{H} & -1.20833400 & 3.09319500 & -0.38709400 \\ \mathrm{H} & -1.37117600 & 2.53916500 & 1.26845800 \\ \mathrm{H} & 5.75176100 & 1.97474900 & 0.02298700 \\ \mathrm{H} & 5.66023800 & 0.95309100 & 1.45404700 \\ \mathrm{H} & 5.86743500 & -0.04089000 & -1.42603000 \\ \mathrm{H} & 9.92622100 & 0.00063800 & 0.32120100\end{array}$

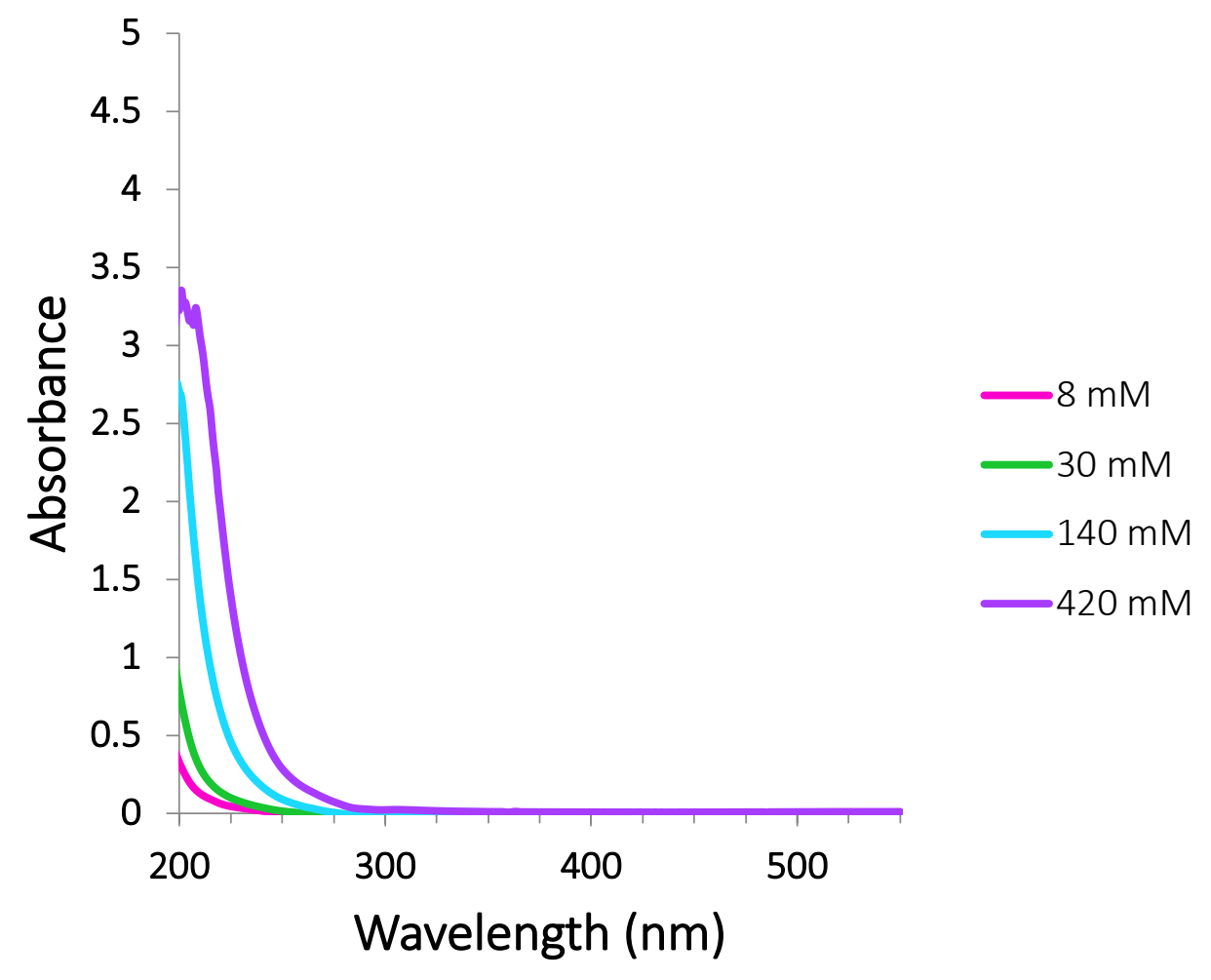

Figure S84. UV-vis spectra of Selectfluor in MeCN at varying concentrations. 


\section{References}

1 Naumann, D; Kischkewitz, J. Trifluormethylierungsreaktionen von $\mathrm{Te}\left(\mathrm{CF}_{3}\right)_{2}$ Mit Halogenbenzolen und Methylbenzolen. J. Fluorine Chem. 1990, 47, 283-299.

${ }^{2}$ Frisch, M. J.; Trucks, G. W.; Schlegel, H. B.; Scuseria, G. E.; Robb, M. A.; Cheeseman, J. R.; Scalmani, G.; Barone, V.; Mennucci, B.; Petersson, G. A.; Nakatsuji, H.; Caricato, M.; Li, X.; Hratchian, H. P.; Izmaylov, A. F.; Bloino, J.; Zhang, G.; Sonnenberg, J. L.; Hada, M.; Ehara, M.; Toyota, K.; Fukuda, R.; Hasegawa, J.; Ishida, M.; Nakajima, T.; Honda, Y.; Kitao, O.; Nakai, H.; Vreven, T.; Montgomery, J. A., Jr.; Peralta, J. E.; Ogliaro, F.; Bearpark, M.; Heyd, J. J.; Brothers, E.; Kudin, K. N.; Staroverov, V. N.; Kobayashi, R.; Normand, J.; Raghavachari, K.; Rendell, A.; Burant, J. C.; Iyengar, S. S.; Tomasi, J.; Cossi, M.; Rega, N.; Millam, J. M.; Klene, M.; Knox, J.e.; Cross, J. B.; Bakken, V.; Adamo, C.; Jaramillo, J.; Gomperts, R.; Stratmann, R. E.; Yazyev, O.; Austin, A. J.; Cammi, A. R.; Pomelli, C.; Ochterski, J. W.; Martin, R. L.; Morokuma, K.; Zakrzewski, V. G.; Voth, G. A.; Salvador, P.; Dannenberg, J. J.; Dapprich, S.; Daniels, A. D.; Farkas, Ö.; Foresman, J. B.; Ortiz, J. V.; Cioslowski, J.; Fox, D. J. Gaussian 09, Revision E.01; Gaussian, Inc., Wallingford, CT, 2009.

${ }^{3}$ Becke, A. D. Density-functional Thermochemistry. III. The Role of Exact Exchange. J. Chem. Phys. 1993, 98, 5648-5652.

${ }^{4}$ Lee, C.; Yang, W.; Parr, R. G. Development of the Colle-Salvetti Correlation-Energy Formula into a Functional of the Electron Density. Phys. Rev. B 1988, 37, 785-789.

${ }^{5}$ González, C; Schlegel, H. B. Reaction Path Following in Mass-Weighted Internal Coordinates. J. Phys. Chem. 1990, 94, 5523-5527.

${ }^{6}$ Fukui, K. The Path of Chemical Reactions - The IRC Approach. Acc. Chem. Res. 1981, 14, 363368.

${ }^{7}$ Cancès, E.; Mennucci, B.; Tomasi, J. A New Integral Equation Formalism for the Polarizable Continuum Model: Theoretical Background and Applications to Isotropic and Anisotropic Dielectrics. J. Chem. Phys. 1997, 107, 3032-3041.

${ }^{8}$ Legault, C. Y. CYLview, version 1.0b; Universite de Sherbrooke: Quebec, Canada, 2009, http://www.cylview.org.

${ }_{9}^{9}$ Dennington, R.; Keith, T.; Millam, J. GaussView, version 5; Semichem, Inc., Shawnee Mission, 2009.

${ }^{10}$ Schrödinger Release 2019-2: Jaguar; Schrödinger, LLC: New York, 2019.

${ }^{11}$ Nakamura, M.; Hatakeyama, T.; Nakamura, E. $\alpha$-Alkylation of Ketones by Addition of Zinc Enamides to Unactivated Olefins. J. Am. Chem. Soc. 2004, 126, 11820-11825.

${ }^{12}$ Pitts, C. R.; Bume, D. D.; Harry, S. A.; Siegler, M. A.; Lectka, T. Multiple Enone-Directed Reactivity Modes Lead to the Selective Photochemical Fluorination of Polycyclic Terpenoid Derivatives. J. Am. Chem. Soc. 2017, 139, 2208-2211.

13 Laali, K. K.; Jamalian, A.; Zhao, C. Reaction of Selectfluor (F-TEDA-BF4) with Chloromethylated-DABCO Monocation Salts $(\mathrm{X}=\mathrm{BF} 4, \mathrm{NTf} 2)$ and Other Nitrogen Bases (Et3N; Piperidine; Basic Ionic Liquid); Unexpected Formation of Symmetrical $[\mathrm{N}-\mathrm{H}-\mathrm{N}]+$ Trication Salts. Tetrahedron Lett. 2014, 55, 6643-6646.

${ }^{14}$ Banks, R. E.; Mohialdin-Khaffaf, S. N.; Lai, G. S; Sharif, I.; Syvret, R. G. 1-Alkyl-4-fluoro-1,4diazoniabicyclo[2.2.2] octane Salts: A Novel Family of Electrophilic Fluorinating Agents. $J$. Chem. Soc., Chem. Commun. 1992, 8, 595-596. 
${ }^{15}$ Banks, R. E; Besheesh, M. K.; Mohialdin-Khaffaf, S. N.; Sharif, I. N-Halogeno Compounds. Part 18. 1-Alkyl-4-fluoro-1,4-diazoniabicyclo[2.2.2]octane Salts: User-Friendly Site-Selective Electrophilic Fluorinating Agents of the N-fluoroammonium Class. J. Chem. Soc., Perkin Trans. 1 1996, 16, 2069-2076.

${ }^{16}$ Cismesiaa, M. A.; Yoon, T. P. Characterizing Chain Processes in Visible Light Photoredox Catalysis. Chem. Sci. 2015, 6, 5426-5434. 